\title{
Opportunities and challenges in the use of inorganic fullerene-like nanoparticles to produce advanced polymer nanocomposites
}

\author{
Mohammed Naffakh ${ }^{\mathrm{a}, \mathrm{b}, *}$, Ana M. Díez-Pascual ${ }^{\mathrm{b}}$, Carlos Marco $^{\mathrm{b}}$, \\ Gary J. Ellis ${ }^{b}$, Marián A. Gómez-Fatou ${ }^{b}$ \\ a Universidad Politécnica de Madrid, Departamento de Ingeniería y Ciencia de los Materiales, Escuela Técnica Superior de Ingenieros \\ Industriales, José Gutiérrez Abascal 2, 28006 Madrid, Spain \\ ${ }^{\mathrm{b}}$ Instituto de Ciencia y Tecnología de Polímeros (ICTP-CSIC), Juan de la Cierva 3, 28006 Madrid, Spain
}

\section{Keywords:}

Inorganic fullerene-like (IF) nanoparticles

Polymer nanocomposites

Thermal, mechanical and tribological

properties

\section{A B S T R A C T}

Polymer/inorganic nanoparticle nanocomposites have garnered considerable academic and ind ustrial interest over recent decades in the development of advanced materials for a wide range of applications. In this respect, the dispersion of so-called inorganic fullerene-like (IF) nanoparticles, e.g., tungsten disulfide (IF-WS 2 ) or molybdenum disulfide (IF-MoS $\mathrm{S}_{2}$, into polymeric matrices is emerging as a new strategy. The surprising properties of these layered metal dichalcogenides such as high impact resistance and superior tribological behavior, attributed to their nanoscale size and hollow quasi-spherical shape, open up a wide variety of opportunities for applications of these inorganic compounds. The present work presents a detailed overview on research in the area of IF-based polymer nanocomposites, with special emphasis on the use of IF-WS $\mathrm{S}_{2}$ nanoparticles as environmentally friendly reinforcing fillers. The incorporation of IF particles has been shown to be efficient for improving thermal, mechanical and tribological properties of various thermoplastic polymers, such as polypropylene, nylon-6, poly(phenylene sulfide), poly(ether ether ketone), where nanocomposites were fabricated by simple melt-processing routes without the need for modifiers or surfactants. This new family of nanocomposites exhibits similar or enhanced performance when compared with nanocomposites that incorporate

Abbreviations: AA, acrylic acid; APTES, $\gamma$-aminopropyltriethoxy silane; BT, barium titanate; $C A$, carboxylic acid; CF, carbon fiber; CFRPs, carbon fiberreinforced polymer composites; $\mathrm{CNO}$, carbon nanoonion; $\mathrm{CNTs}$, carbon nanotubes; COF, coefficient of friction; DCMS, dichlorodimethylsilane; DCP, dicumyl peroxide; DMA, dynamic mechanical analysis; DHBP, 2,5-dimethyl-2,5(tert-butylperoxy)hexane peroxide; EVA, ethylene-co-vinyl acetate; GPTMS, glycidyloxypropyltrimethoxy silane; HMDS, hexamethyldisilazane; HPMC, hydroxyl propyl methyl cellulose; HRSEM, high-resolution scanning electron microscopy; IF, inorganic fullerene-like; IF-MoS 2 , inorganic fullerene-like molybdenum disulfide nanoparticles; IF-WS disulfide nanoparticles; I-PNCs, inorganic polymer nanocomposites; IF-PNCS, polymer nanocomposites based on IF nanopart icles; iPP, isotactic polypropylene; $\mathrm{KH}-550$, trialkoxysilane coupling agent; $\mathrm{KH}-560, \gamma$-glycidyl propyl trimethoxyl silicane; $\mathrm{KH}-570, \gamma$-metacryloxypropyl trimethoxysilane; MA, malonic acid; MAH, maleic anhydride; MWCNTs, multi-walled carbon nanotubes; $\mathrm{NA}$, nucleating agent; $\mathrm{NE}$, nucleation efficiency; $\mathrm{NH}_{2}-\mathrm{A}$, amino acid; $\mathrm{NJ}$, $\mathrm{N}, \mathrm{N}^{\prime}-$ dicyclohexyl-2,6-naphthalene; $\mathrm{OA}$, oleic acid; OMMT, organo montmorillonite; Organo $\mathrm{CH}_{3}, 1,3,5$-benzene tricarboxylic-( $\mathrm{N}$-2-methylcyclohexyl)triamine; PA, polyamide (nylon); PBA, poly(butyl acrylate); PCL, poly(e-caprolactam); PEEK, poly(ether ether ketone); PEI, polyetherimide; PGMA, poly(glycidyl methacrylate); PNCs, polymer nanocomposites; POE, polyolefin elastomer; POM, polymethylene oxide; POSS, polyhedral oligomeric silsesquioxane; PP, polypropylene; $\mathrm{PP}-\mathrm{g}-\mathrm{MAH}$, maleic anhydride-grafted polypropylene; $\mathrm{PP}-\mathrm{g}-\mathrm{NH}_{2}$, aminated polypropylene; PPS, poly(phenylene sulphide); $\mathrm{PS}$, polystyrene; PTFE, poly(tetrafluoroethylene); PVA, poly(vinyl alcohol); SA, stearic acid; SCFs, short carbon fibers; SDBS, sodium dodecylbenzene sulfonate; SEBS, styrene ethylene butylene styrene; SiC, silicon carbide; SPEEK, sulfonated poly(ether ether ketone); SSA, specific surface area; SWCNTs, single-walled carbon nanotubes; $T_{c}$, crystallization peak temperature; $T_{c, c}$, cold crystallization temperature; $T_{g}$, glass transition temperature; $T_{\mathrm{i}}$, initial degradation temperature; $T_{10}$, temperature of $10 \%$ of weight loss; $T_{\mathrm{m}}$, melting temperature; $T_{\mathrm{mr}}$, temperature of maximum rate of weight loss; TMPM, trimethoxysilylpropylmethacrylate; UHMWPE, ultra-high-molecular-weight poly(ethylene); $\chi_{c}$, degree of crystallinity; WAXS, wide angle X-ray diffraction.

* Corresponding author at: Universidad Politécnica de Madrid, Departamento de Ingeniería y Ciencia de los Materiales, Escuela Técnica Superior de Ingenieros Industriales, José Gutiérrez Abascal 2, 28006 Madrid, Spain. Tel.: +34 913363 164; fax: +34 913363007.

E-mail address: mohammed.naffakh@upm.es (M. Naffakh). 
carbon nanotubes, carbon nanofibers or nanoclays, but are substantially more costeffective, efficient and environmentally satisfactory. Most recently, innovative approaches have been described that exploit synergistic effects to produce new materials with enhanced properties, including the combined use of micro- and nanoparticles such as IF-WS 2 /nucleating agent or IF-WS 2 /carbon fiber, as well as dual nanoparticle systems such as SWCNT/IF-WS 2 where each nanoparticle has different characteristics. The structure-property relationships of these nanocomposites are discussed and potential applications proposed ranging from medicine to the aerospace, automotive and electronics industries.

\section{Contents}

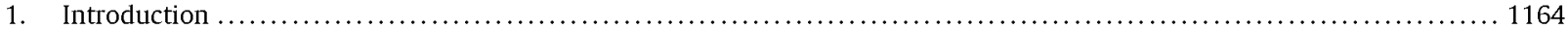

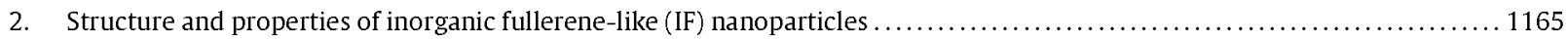

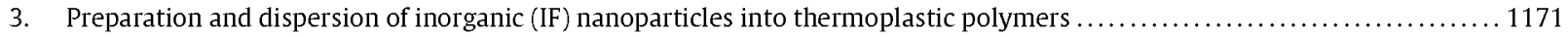

4. Thermoplastic polymer nanocomposites based on inorganic fullerene-like nanoparticles (IF-PNCs) $\ldots \ldots \ldots \ldots \ldots \ldots \ldots \ldots 173$

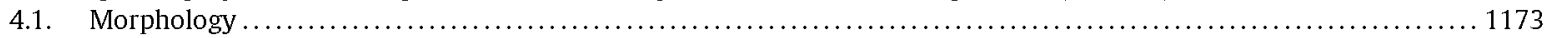

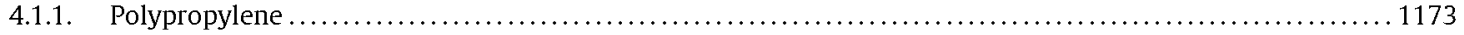

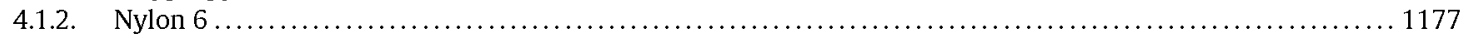

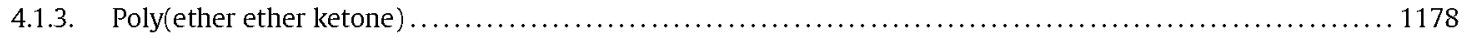

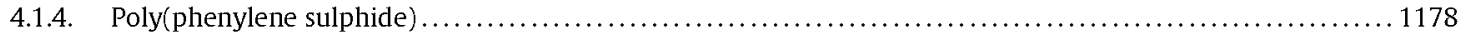

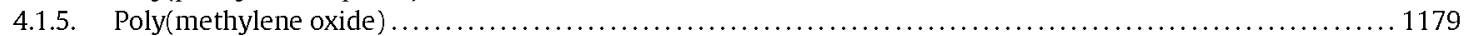

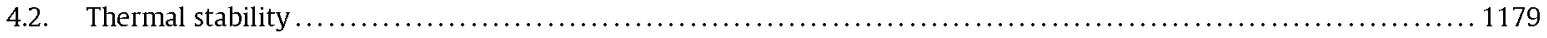

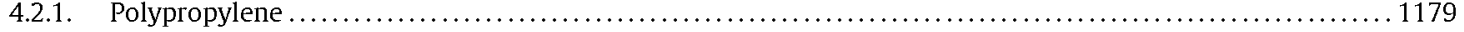

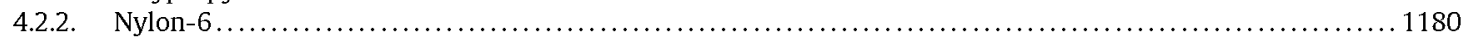

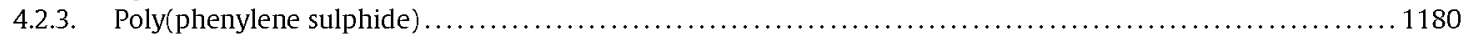

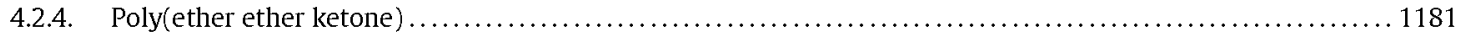

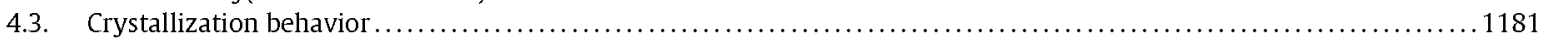

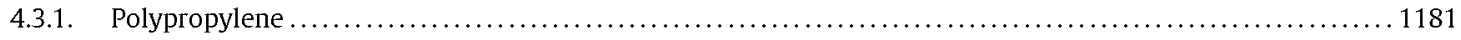

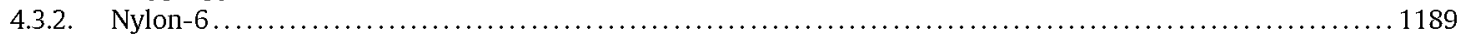

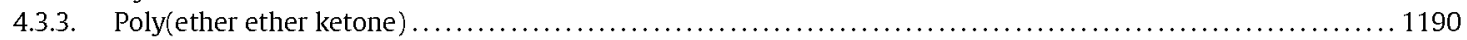

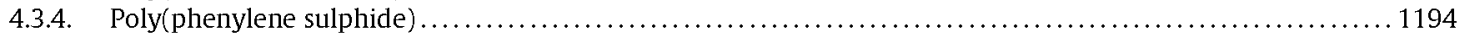

4.3.5. Polymethylene oxide ..................................................................... 1194

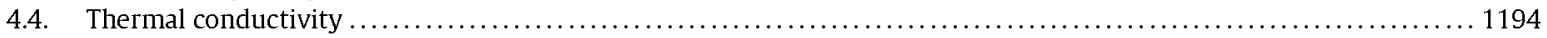

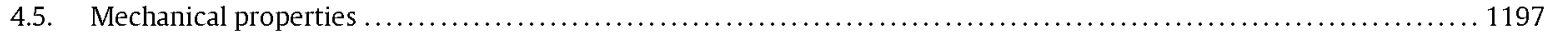

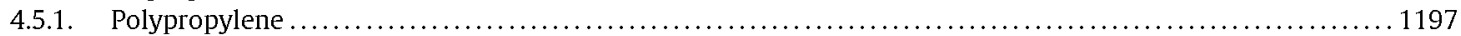

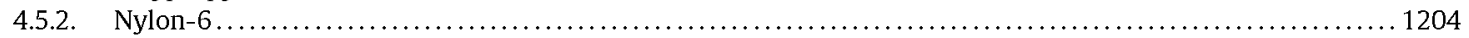

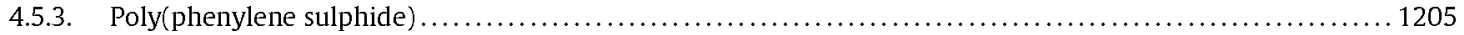

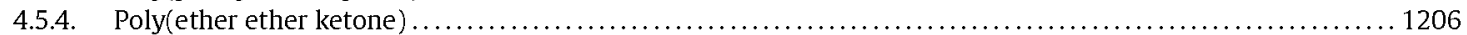

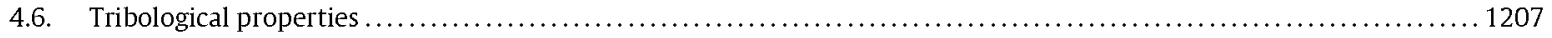

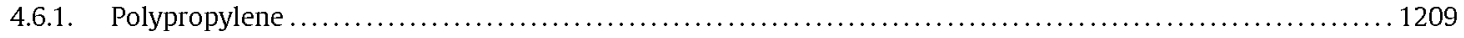

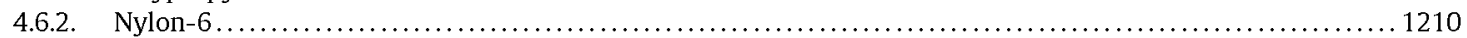

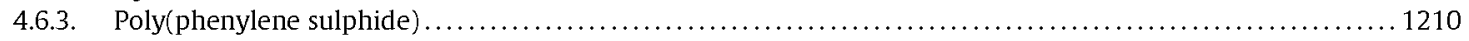

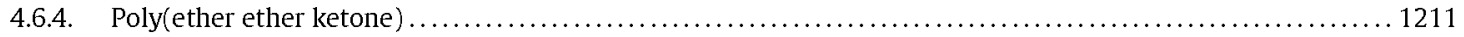

5. Novel dual filler strategies for hybrid thermoplastic polymer nanocomposites based on inorganic

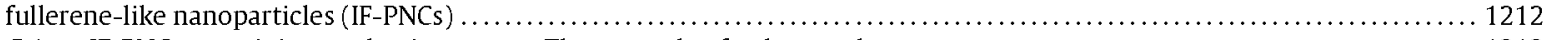

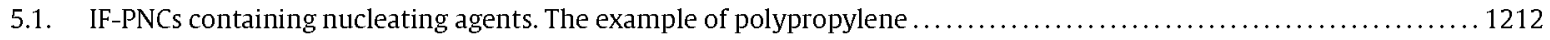

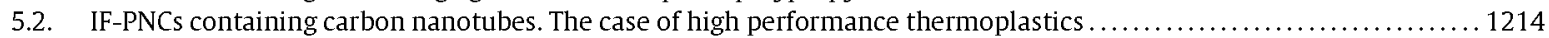

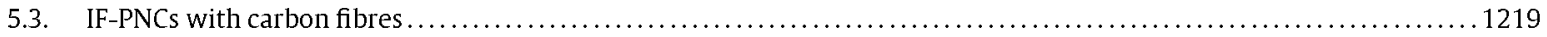

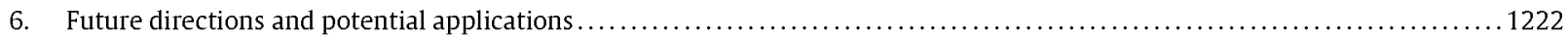

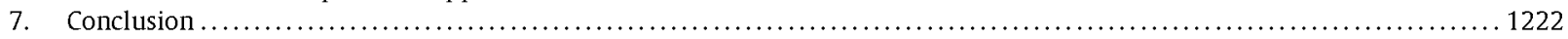

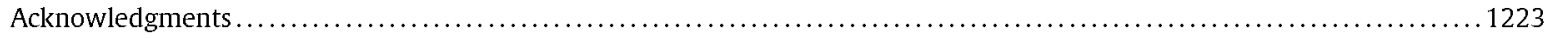

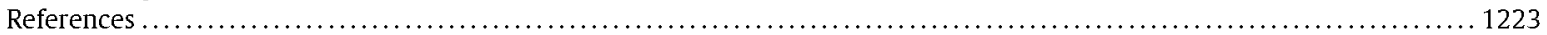

\section{Introduction}

Organic-inorganic hybrid materials are found at the natural interface between two worlds of chemistry each with very significant contributions to the field of materials science, and with characteristic properties that present numerous advantages. It is clear today that hybrid organic-inorganic materials will play a major role in the development of advanced functional nanomaterials. Hybrid organic-inorganic nanomaterials can be broadly defined as containing both organic and inorganic components that are intimately mixed, and where at least one of the component domains has dimensions ranging 
within the nanometer scale [1-5]. In particular, hybrid polymer nanocomposites (PNCs) can be described as the combination of a polymer matrix with inorganic or hybrid nanoscale objects [6]. The development of new materials based on PNCs has been a fast-growing field of research over the last decade. In these nanocomposites, significant polymer property enhancement can be achieved at much lower filler loading ( $\leq 10 \mathrm{wt} . \%)$ than those obtained with traditional microscale fillers, simultaneously improving material processing and reducing component weight. The polymer-nanofiller composite properties are determined by the characteristics of the nanofillers, their dispersion and their interaction with the polymer matrix [7]. Some advantages of nanoscale fillers are their high specific surface area (SSA), which can act as a stress-transfer interface, and their aspect ratio, which affects the percolation threshold [8]. In this respect, various types of nanofillers are being employed, including fillers based on carbon, including carbon black, fullerenes, nanotubes and graphene [8-12], as well as inorganic particles including silica $\left(\mathrm{SiO}_{2}\right)$, metal oxides $\left(\mathrm{ZnO}, \mathrm{Al}_{2} \mathrm{O}_{3}, \mathrm{CaCO}_{3}, \mathrm{TiO}_{2}\right.$, etc.), metallic phosphates $\left(\mathrm{Zr}\left(\mathrm{HPO}_{4}\right)\right.$ ), clays (montmorillonite, hectorite, saponite), metals (Al, Fe, Au, Ag, etc.), silicon carbide (SiC), and transition metal chalcogenides $\left(\mathrm{WS}_{2}\right.$, $\mathrm{MoS}_{2}$, etc.), amongst others [4-8,13-16]. The nanoparticle selection depends on the target properties of the nanocomposite (thermal, mechanical, tribological, optical, magnetic and optoelectronic, etc.). For example, Al nanoparticles are often selected due to their high conductivity, $\mathrm{CaCO}_{3}$ particles because of the relative low cost of the composite material, and $\mathrm{SiC}$ nanoparticles because of their high hardness, corrosion resistance, and strength. Consequently, the main idea when developing hybrid materials is to take advantage of the best properties of each component of the hybrid with a view to decrease or eliminate their drawbacks and, ideally, obtain synergetic effects that allows the development of new materials with new or improved properties. Due to their wide component versatility, PNCs find broad applications in various fields such as military equipment, safety, protective clothing, automotive, aerospace, electronics, optical and medical devices. However, these application areas continuously demand additional properties and functionality in particular high mechanical properties, flammability, chemical resistance, UV resistance, electrical conductivity, environmental stability, water repellence, magnetic field resistance, radar absorption, etc.

Recently, inorganic fullerene-like (IF) nanoparticles have emerged as one of the most promising developments in the area of nanomaterials. These nanofillers can offer significant advantages over other spherical nanoparticles for the preparation of advanced PNCs. The aim of this review is to highlight the latest findings on the effect of these inorganic nanoparticles on the structure, morphology and properties of thermoplastic polymer nanocomposites, in comparison with PNCs based on other spherical inorganic nanoparticles. Special emphasis has been placed on the thermal, mechanical and tribolological property enhancements achieved with IF nanoparticles. Finally, a new generation of hybrid nanocomposites employing dual filler strategies is discussed.

\section{Structure and properties of inorganic fullerene-like (IF) nanoparticles}

The discovery of soccer-ball shaped Buckminsterfullerene in 1985 [17] was an exciting and unexpected discovery that established an entirely new branch of chemistry, recognized by the award of the Nobel Prize in Chemistry to Harry Kroto, Richard Smalley and Robert Curl in 1996. Fullerenes are still the subject of intensive research and present a myriad of important properties including superconductivity at relatively high temperature [18], very high mechanical stability, transformation into diamond under pressure, ferromagnetism and a variety of unique chemical reactions [19]. This remarkable discovery has led to exciting new avenues of multidisciplinary research in chemistry, physics, materials science and even biology. Soon after the discovery of fullerenes, the first fullerenecontaining polymer was synthesized and a new type of material appeared that combined the processability, ease of handling and toughness of polymers with the unique geometrical and electronic properties of fullerenes. Olah [20] reported the first example of a polyfullerene material, prepared via a Friedel-Crafts reaction of a $C_{60} / C_{70}$ mixture with monodispersed polystyrene (PS) using an $\mathrm{AlCl}_{3}$ catalyst. Since then, a number of different $\mathrm{C}_{60}$-polymer systems have been prepared and are widely reviewed $[9,21]$. Whilst research was advancing on these carbon structures, other researchers were investigating the possibilities of fullerenes and fullerene-like materials composed of elements other than carbon.

Inorganic layered compounds are abundant, in particular among the transition-metal chalcogenides (sulfides, selenides, and tellurides), halides (chlorides, bromides, and iodides), oxides, and numerous ternary and quaternary compounds $[22,23]$. It was shown that, in analogy to carbon fullerenes, nanoparticles of such compounds suffer from inherent chemical instability in the planar form due to their abundant rim atoms that can be alleviated by folding to form seamless hollow nanoparticles that are, nevertheless, elastically strained. This type of nanoparticle is currently the subject of intense research, summarized in recent reviews that include synthetic methodologies, diverse properties of these new nanomaterials and their potential applications [23-26].

One group working on non-carbon structures analogous to fullerenes, led by Reshef Tenne at the Weizmann Institute in Rehovot, Israel, were the first to suggest and observe in 1992 that $\mathrm{WS}_{2}$ (and $\mathrm{MoS}_{2}$ ) are able to form closed-cage structures like those formed by carbon $[27,28]$. However, these structures are not perfectly analogous to carbon fullerenes, and the Israeli group has been careful to call their materials "fullerene-like". In the case of the layered semiconductor $\mathrm{WS}_{2}$ the transmission electron micrographs in Fig. 1 show lattice images of two typical polyhedral growth forms, spherical (Fig. 1a) and elongated (Fig. 1b). Such crystallites occasionally occur in $\mathrm{WS}_{2}$ films obtained by annealing on quartz substrates at $1000^{\circ} \mathrm{C}$ in $\mathrm{H}_{2} \mathrm{~S}$ in a reducing atmosphere. Fig. 1c shows the range of shapes that can appear, and also demonstrates that these structures occur fairly frequently with the layers. Further evidence for the three-dimensional curvature of the 

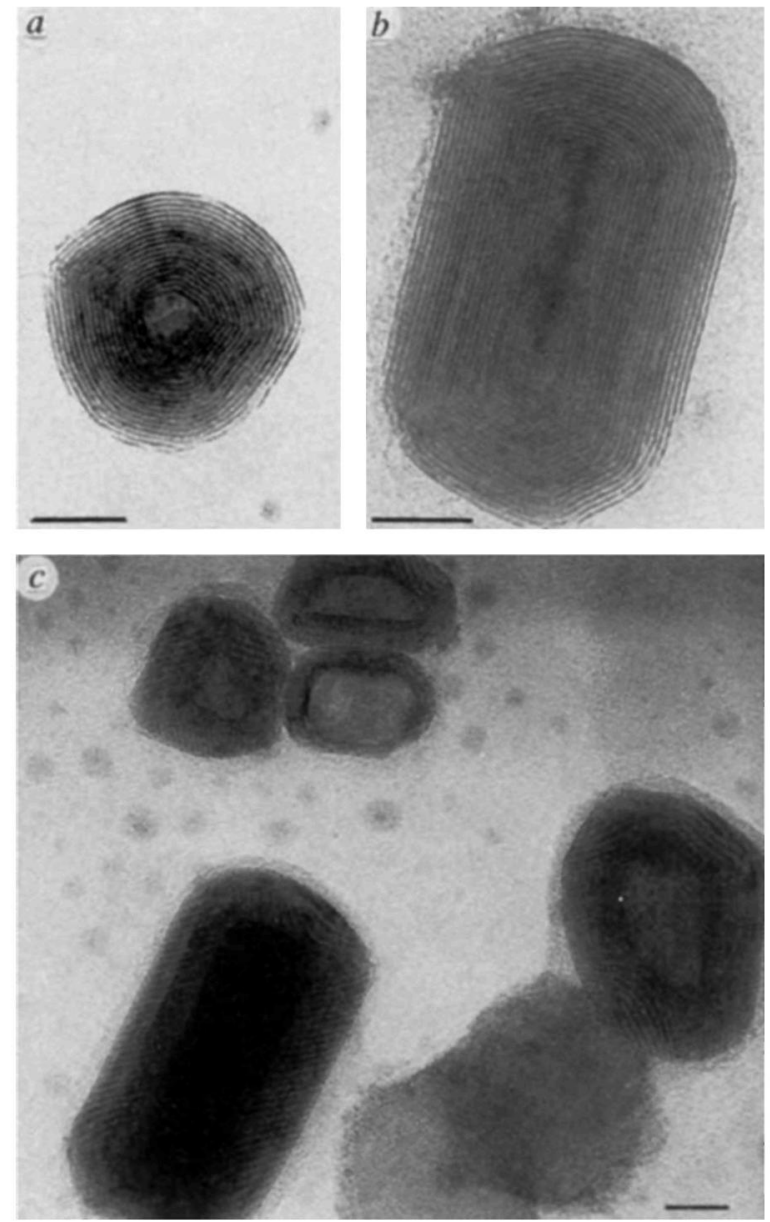

Fig. 1. Electron micrographs of polyhedral crystal of $\mathrm{WS}_{2}$. (a) Spheroidal crystal, (b) stir-bar crystal and (c) region containing several polyhedral crystals. The spacing between fringes is $0.615 \pm 0.005 \mathrm{~nm}$, which agrees with the distance between two neighboring layers $(c / 2)$ of $\mathrm{WS}_{2}$. Two platelike crystals partially overlapping are also seen at the bottom.

Reprinted with permission [27]. Copyright 1992, Macmillan Publishers Ltd: Nature.

crystals was also confirmed by typical single-crystal electron diffraction patterns [27]. Such concentric structures were also observed for $\mathrm{MoS}_{2}$ [28] where molybdenum films of $20 \mathrm{~nm}$ thickness were deposited onto quartz substrates, oxidized at $500-600^{\circ} \mathrm{C}$ in open air and fired at $850-1050^{\circ} \mathrm{C}$ in a stream of $\mathrm{H}_{2} \mathrm{~S}$ and a mixture of $\mathrm{N}_{2} / \mathrm{H}_{2}$.

Both graphitic carbon and molybdenum sulfide are layered structures. Graphite is made up of layers of $\mathrm{sp}^{2}$ carbon sheets resembling chicken wire, stacked in a staggered form one layer upon the other, with strong bonds between carbon atoms within each layer and weaker van der Waals interactions holding the layers together. $\mathrm{MoS}_{2}$ has a similar structure, and each stacking layer consists of sulphur-molybdenum-sulphur groups, Fig. 2 [29] where each Mo atom binds to six sulfur atoms to form a trigonal biprism. In analogy to graphite, weak van der Waals forces are responsible for the stacking of the S-Mo-S layers, and they are also highly anisotropic with respect to many of their physical and chemical properties. The basal (van der Waals) surfaces of the crystal, which

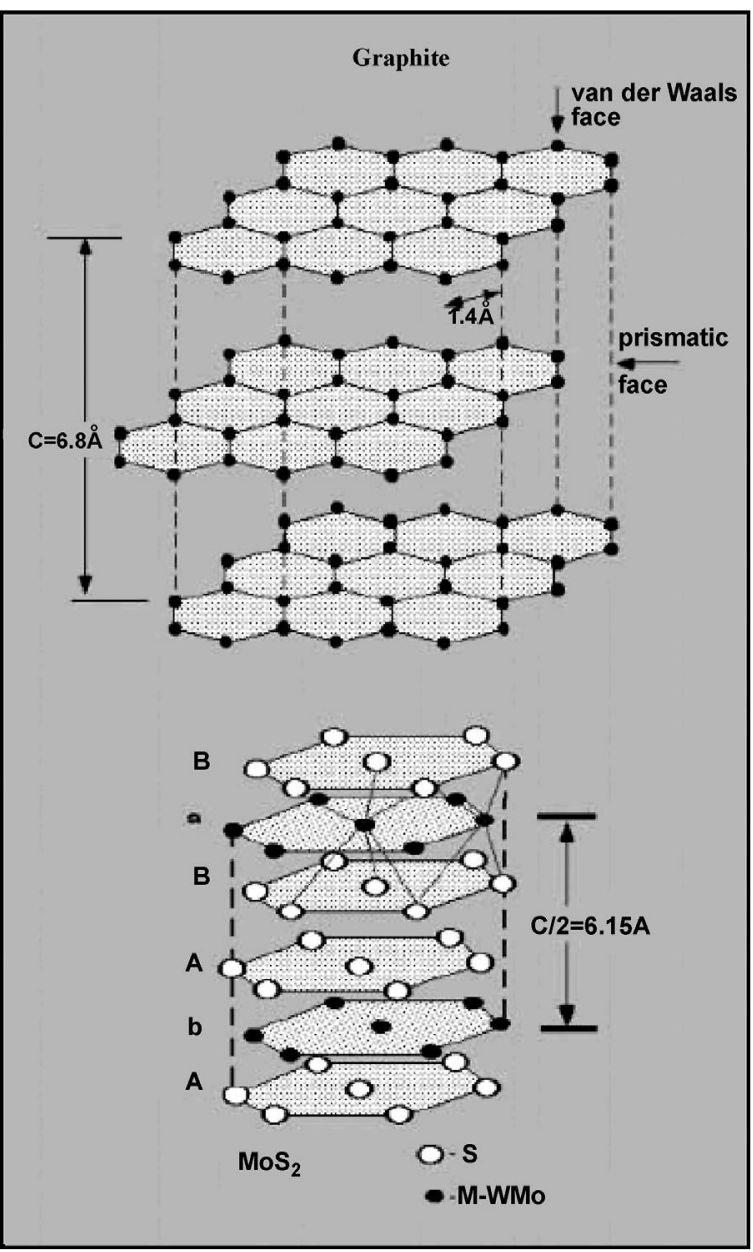

Fig. 2. Schematic drawings of graphite and $\mathrm{MoS}_{2}\left(\mathrm{WS}_{2}\right)$ nanoclusters. Note that in both cases, the surface energy, which destabilizes the planar topology of the nanocluster, is concentrated in the prismatic edges parallel to the $c$ axis.

Reprinted with permission [29]. Copyright 2002, John Wiley and Sons.

are perpendicular to the $c$-axis, consist of sulfur atoms that form bonds to three underlying Mo atoms. These sulfur atoms are almost chemically inert. However, rim Mo and $\mathrm{S}$ atoms that abound in the nano-regime are only four- and twofold bonded, respectively, making these nanostructures unstable in the planar form. However, by folding the molecular sheet and stitching the rim atoms together seamless and stable structures can be obtained that are nano-tubular (one dimensional) or spherical (zero-dimensional or three-dimensional), where all Mo and $\mathrm{S}$ atoms are six- and threefold-bonded, respectively.

Under a shearing force the basal planes can slide over one another by intracrystalline slip and transfer to the rubbing counterface conferring excellent lubricating properties. The slippery nature of graphite is also well known to those who have rubbed "pencil lead" between their fingers, and its appearance highlights another similarity with molybdenum sulfide, namely, that it is also black. Indeed, molybdenum sulfide and graphite were originally thought to be the same material, and were also confused with 

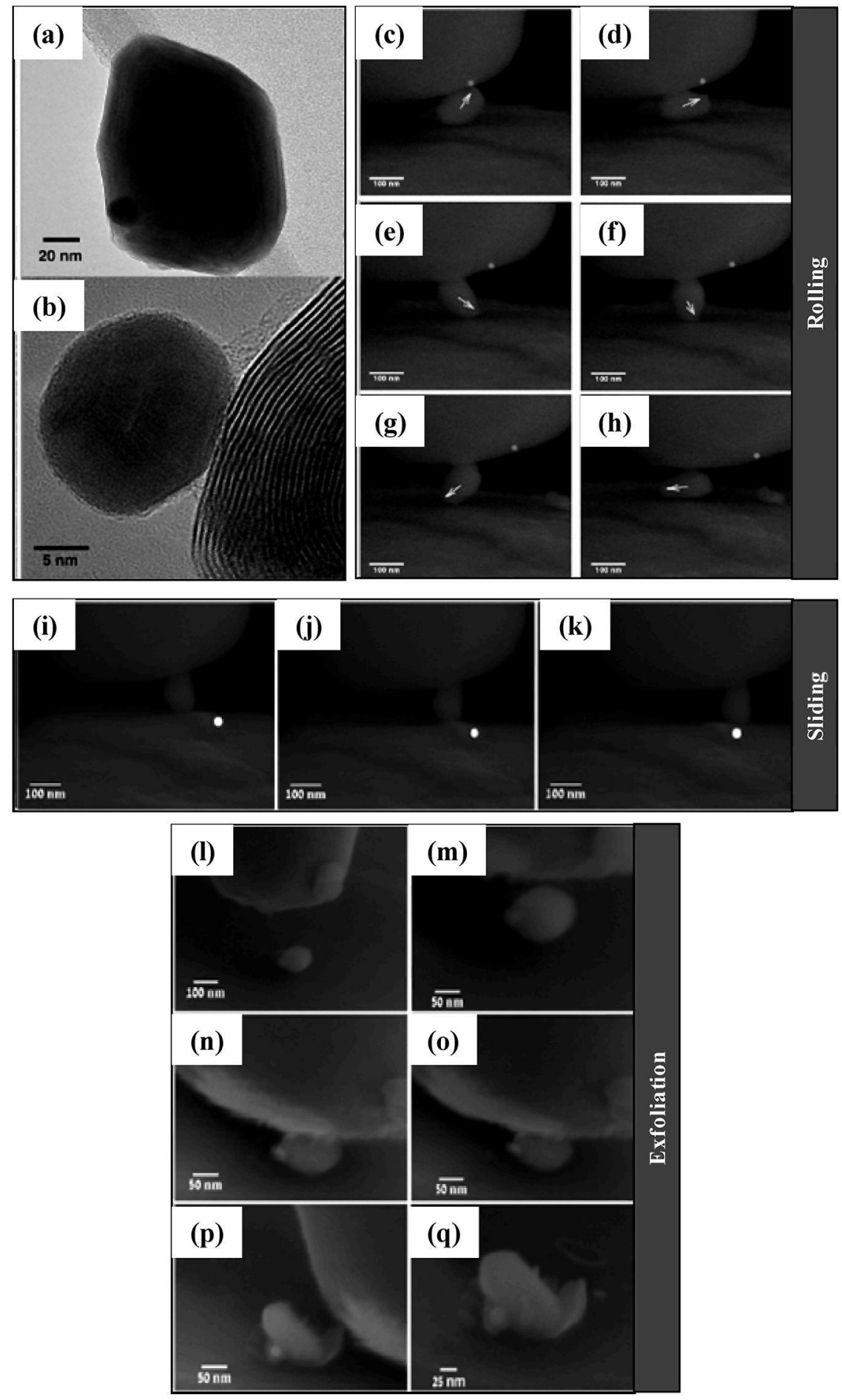

Fig. 3. (a) TEM image of an IF-WS 2 nanoparticle decorated by a gold nanoparticle from the aqueous solution and (b) HRTEM images of gold nanoparticle attached to an IF-WS $S_{2}$ nanoparticle; (c) to $(\mathrm{h})$ show the rolling of an IF-WS 2 nanoparticle decorated with GNP. The arrow points out the GNP attached to the rolling IF-WS 2 nanoparticle. The blue dot marks the starting point on the AFM tip; (i) to (k) show the sliding of an IF-WS 2 nanoparticle along a distance of $170 \mathrm{~nm}$. The white dot points out the sliding onset on the Si wafer and (L) to (q) show the demonstration of an ex foliation mechanism: (I) and (m) show image of the flat AFM tip above the WS 2 nanoparticle. (n) and (o) Show uniaxial pressing of the WS ${ }_{2}$ nanoparticle by the AFM tip under loads of 0.7 and $1.2 \mathrm{GPa}$, respectively. (p) and (q) Show the exfoliated IF nanoparticle in two magnifications. The AFM tip is seen at the right side of the nanoparticle (p). In this experiment combined normal and shear stresses were applied to the nanoparticle by the AFM tip. Adapted with permission [34]. Copyright 2011, National Academy of Sciences, USA. 

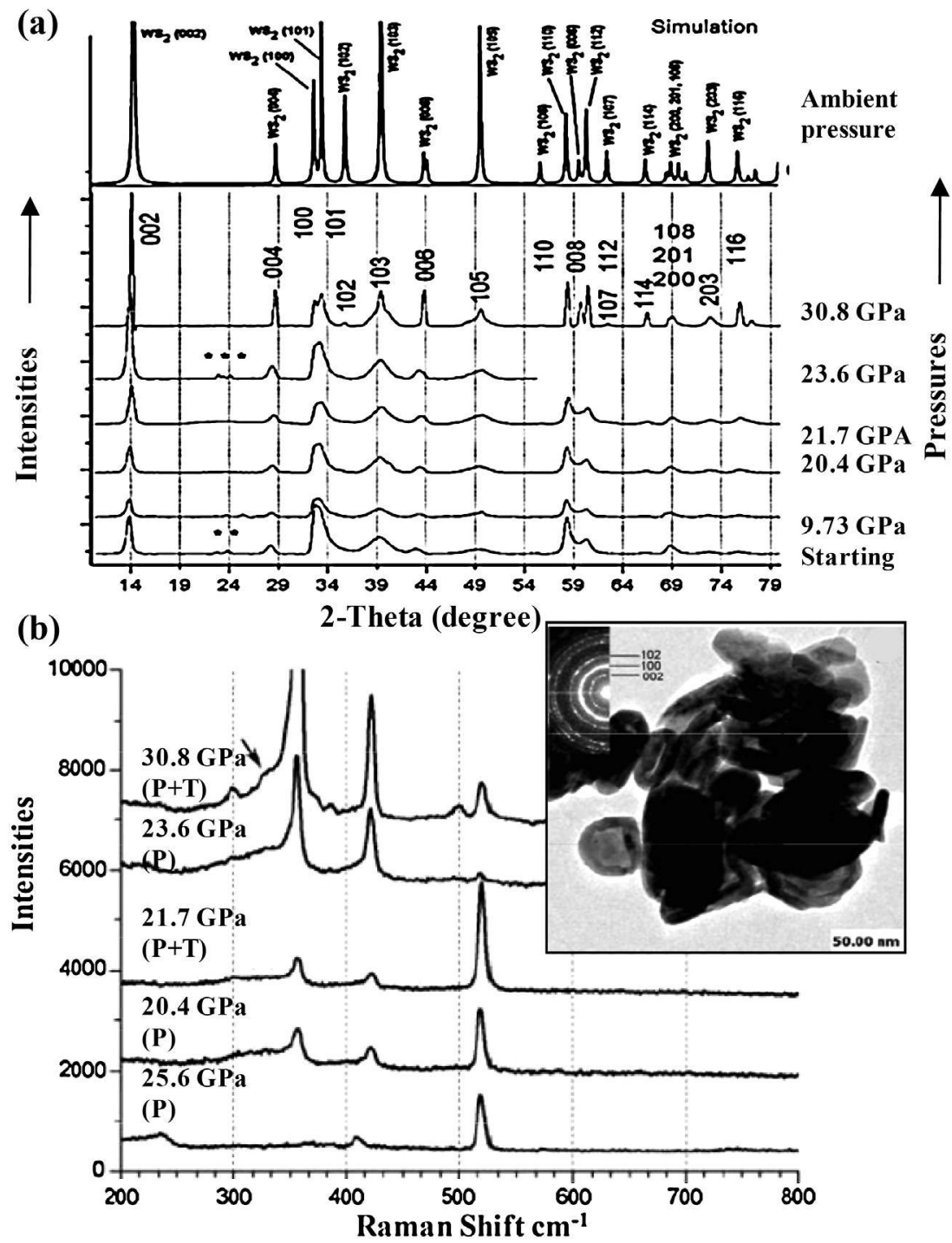

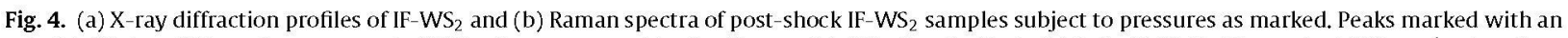

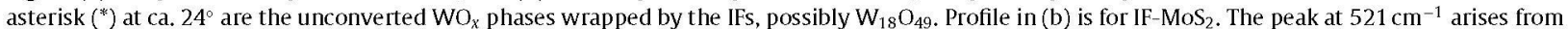
the Si substrate.

Adapted with permission [36]. Copyright 2005, American Chemical Society.

black lead until, in 1778, the Swedish chemist Karl Wilhelm Scheele experimented with a black powder and found that it contained molybdenum. In fact, it was the similarity of molybdenum sulfide to black lead that gave molybdenum its name; the word molybdae, meaning leadlike, was applied to these materials by Dioscurides, a Greek pharmacist, and used by the Roman scholar Pliny the Elder. It was in 1923 that Linus Pauling, in his first research paper, showed that $\mathrm{MoS}_{2}$ has a layered structure analogous to that of graphite [30].

It was reported that the major obstacle to lubrication by metal dichalcogenides is due to the presence of unsaturated or dangling bonds. For example, in a typical layered structure of hexagonal tungsten disulphide $\left(2 \mathrm{H}-\mathrm{WS}_{2}\right)$, the obvious source of dangling bonds is an edge plane. In particular, the propagation of a pre-existing crack in a $W_{2}$ film will break a few covalent bonds and create more unsaturated bonds. If sliding takes place in humid air, such activated surfaces can instantly react with moisture and oxygen in the surrounding environment, forming $\mathrm{WO}_{3}$. Thus, the tribological (friction and wear) behavior of metal dichalcogenides is strongly dependent on their environment [31]. When the test environment is switched from dry nitrogen to humid air [32], the coefficient of friction (COF) of a typical $\mathrm{WS}_{2}$ film can rise over five-fold, considerably decreasing its wear life. In contrast, graphite loses its lubricating property in a vacuum since the complete absence of adsorbed vapors makes it difficult to shear the layers. Rapoport et al. reported that hollow IF-WS 2 nanoparticles were found to have excellent lubricating properties [33]. This behavior can be explained by the superposition of three main mechanisms: rolling, sliding, and exfoliation-material transfer. In order to elucidate the tribological mechanism of individual nanoparticles in 
different regimes, in situ axial nanocompression and shearing forces were applied to individual nanoparticles using a high resolution scanning electron microscope (HRSEM), Fig. 3. Gold nanoparticles were attached to the IF nanoparticles which served as markers relative to the substrate. It can be concluded from the experiments of Tevet et al. [34] that rolling is an important lubrication mechanism for IF-WS 2 in the relatively low range of normal stress $(0.96 \pm 0.38 \mathrm{GPa})$. Sliding is shown to be relevant under slightly higher normal stress, where the spacing between the two mating surfaces does not permit free rolling of the nanoparticles. Exfoliation of the IF nanoparticles becomes the dominant mechanism at the high end of normal stress; above $1.2 \mathrm{GPa}$ and (slow) shear, i.e. boundary lubrication conditions.

The tribological properties of these nanomaterials can also be intimately related to their mechanical performance. Recent work by Zhu et al. show that IFs are superb anti-shock materials with the layered cage-like structure contributing to their excellent shock-absorbing properties $[35,36]$. With the majority of these IF nanostructures surviving approximately $25 \mathrm{GPa}$ of non-uniaxial dynamic pressure, they suggest that these IFs are probably the toughest cage molecules known (for example, the interlayer separation of IFs is 0.62 compared to $0.34 \mathrm{~nm}$ for carbon nanotubes, CNTs), and are superior to all-carbon structures that collapse and are converted to diamond under similar or much lower pressures. Wide angle X-ray diffraction (WAXD) spectra obtained from post-shock samples, Fig. 4a, show only minor changes in their structural integrity at around $10 \mathrm{GPa}$ when compared to the starting material, and below $25 \mathrm{GPa}$ only a slight increase in these changes are observed, while at $30.8 \mathrm{GPa}$ the XRD profile is very similar to that of bulk $2 \mathrm{H}-\mathrm{WS}_{2}$ crystals. A simulation pattern for $\mathrm{WS}_{2}$ nanoparticles at ambient pressure agrees that the majority of IFs are collapsed at this pressure. However, the results indicated that caged $\mathrm{WS}_{2}$ samples are able to sustain applied pressures of up to $25 \mathrm{GPa}$ [36]. A Raman structural study was also performed on the postshock samples to examine the pressure effects on the vibrational response of the cagelike IF samples [36]. WS samples shocked under an applied pressure of 20-30 GPa were compared to the starting IF material, bulk $2 \mathrm{H}$ platelet $\mathrm{WS}_{2}$ powder, and $\mathrm{MoS}_{2}$ powder, Fig. 4b [36]. Only a few spectral peak position and intensity changes were observed and certain frequencies remained identical to the spectra of the starting material and $2 \mathrm{H}$ platelet particles [37], also a strong indication of the superior anti-shock wave performance of IF-MoS $\mathrm{Ms}_{2}$ ampared with that of IF-WS 2 . TEM also revealed that the majority of IFs are capable of sustaining shock pressure up to $25 \mathrm{GPa}$. When localized pressure and temperature are high, IFs have been reported to suffer destruction via two possible separate or combined mechanisms; direct stress-induced breakage failure and diffusion-controlled oxidation [36].

Tevet et al. developed an in situ technique for stiffness measurements on individual $\mathrm{WS}_{2}\left(\mathrm{MoS}_{2}\right)$ nanoparticles [38]. When the experiments were performed in a HRSEM it was possible to elucidate the compression failure strength and the elastic behavior of such nanoparticles under uniaxial compression. Fig. 5 presents a series of load-deformation traces in HRSEM compression tests of

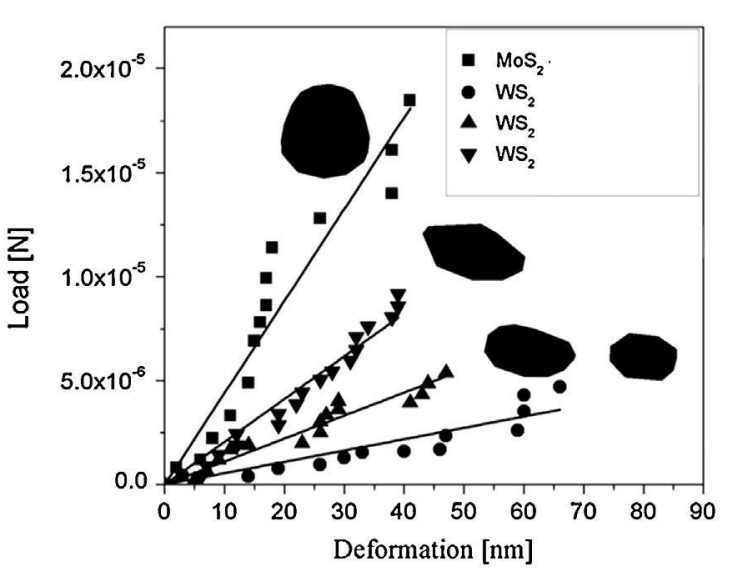

Fig. 5. The load-deformation traces generated in HRSEM compression tests of multilayered polyhedral nanoparticles. The stiffness is obtained by calculating the load/de formation ratio of the linear regression lines. Reprinted with permission [38]. Copyright 2010, Institute of Physics Publishing (IOP)

multilayered polyhedral nanoparticles. Despite the fact that the faceted shape of the nanoparticles leads to the stress being concentrated at the corners, the compression failure strength of these nanoparticles is as high as $1-2.5 \mathrm{GPa}$. The stiffness of the faceted nanoparticles is found to be lower than that of a spherical nanoparticle, due to higher stress concentration at the corners. Furthermore, the Young's modulus of bulk $\mathrm{MoS}_{2}(238 \mathrm{GPa})$ is considerably higher than that of bulk WS $\mathrm{W}_{2}$ (150 GPa) [39]. However, the use of HRSEM, unfortunately, does not offer a good image quality for the observation of any structural modification of the nanoparticles under compressive and/or shear stress. In the following work, compression tests of individual IF-MoS 2 nanoparticles were carried out in a high-resolution transmission electron microscope (HRTEM) using an in situ TEM nanoindenter. Thanks to this technique, the in situ behavior of single nanoparticles under pressure can be observed for the first time [40].

Layered $\mathrm{MS}_{2}(\mathrm{M}=\mathrm{W}, \mathrm{Mo})$ compounds were proposed as a promising material for photovoltaic cells. Frey et al. have reported a comprehensive study of the optical properties of IF-MS $\mathrm{M}_{2}$ materials [41]. Optical-absorption spectroscopy of IF nanoparticles was reported in the range $400-800 \mathrm{~nm}$, at temperatures between 4 and $300 \mathrm{~K}$, and compared to the corresponding bulk $(2 \mathrm{H})$. In the same way, Seifert et al. have studied the electronic properties of $\mathrm{WS}_{2}\left(\mathrm{MoS}_{2}\right)$ nanotubes using the Density Functional Based Tight Binding method (DFTB) [42]. For this other fascinating structure, theory predicts that although the bulk $2 \mathrm{H}-\mathrm{WS}_{2}\left(\mathrm{MoS}_{2}\right)$ material has an indirect band gap, in analogy so would the armchair $\mathrm{WS}_{2}\left(\mathrm{MoS}_{2}\right)$ nanotubes, and the zigzag $\mathrm{WS}_{2}$ $\left(\mathrm{MoS}_{2}\right)$ nanotubes should have a direct band gap. Another feature discovered was that the band gap decreases with diameter size, because of the change in curvature of the S-W-S triple layer. This contrasts with carbon nanotubes and QDs where the bandgap increases with the shrinking size of the nanoparticles because of quantum confinement of the electron (hole). Kopnov et al. have studied the transport properties of IF-WS 2 nanoparticles [43]. Electrical resistivity and Hall effect measurements of pellets 


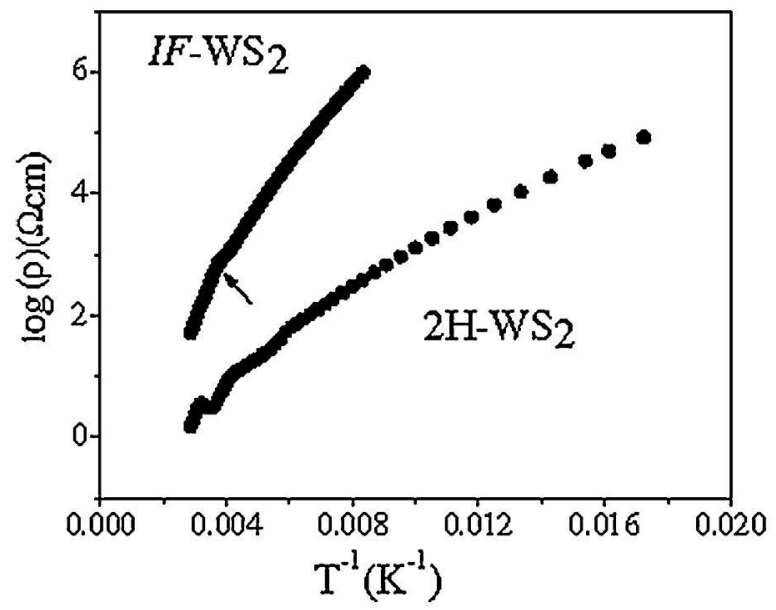

Fig. 6. Temperature dependence of the resistivity for compacted pellets from $2 \mathrm{H}$ and IF-WS 2 powder.

Reprinted with permission [43]. Copyright 2006, John Wiley and Sons.

compacted from IF-WS 2 nanoparticles and bulk $2 \mathrm{H}-\mathrm{WS}_{2}$ powder were carried out using the van der Pauw method over a wide temperature range. Fig. 6 shows that the temperature dependence of the resistivity of either $(2 \mathrm{H}$ and IF) materials exhibit a similar trend. It might be concluded therefore that the mechanism of electrical transport in pellets consisting of nanoparticles and of platelets is similar. However, the resistivity of the IF-WS 2 pellet is higher than that of $2 \mathrm{H}-\mathrm{WS}_{2}$. Furthermore, the graph of the resistivitytemperature dependence of IF-WS 2 is steeper than that for the $2 \mathrm{H}-\mathrm{WS}_{2}$ material. The discrepancies between the resistivities of IF and $2 \mathrm{H}$ pellets probably stem from structural differences between the two materials [43].
In order to study the properties of IFs in greater detail it was necessary to elucidate the growth mechanism of such nanoparticles, which were first formed by sulfidization of their respective amorphous $\mathrm{MO}_{3}$ films on quartz substrates in a reducing atmosphere at temperatures of about $850^{\circ} \mathrm{C}[27,28]$. Indeed, a detailed study of the growth mechanism of IF-WS ${ }_{2}$ was undertaken, which culminated in the synthesis of the first gram of IF-WS ${ }_{2}$ nanoparticles by 1996 and the first kilogram by the end of 2001 . Feldman et al. proposed a model for the growth mechanism of the IF-MS 2 phase from an oxide precursor [44]. Although the syntheses of the IF phases of $\mathrm{MoS}_{2}$ and $\mathrm{WS}_{2}$ differ in some important details, the salient features are common to both materials [29]. A schematic representation of the growth mode of an IF particle is depicted in Fig. 7. First, nanoparticles of the oxide phase are obtained. $\mathrm{MoO}_{3}$ nanoparticles are obtained in situ by evaporating $\mathrm{MoO}_{3}$ powder at $800^{\circ} \mathrm{C}$ and subsequently reducing the vapor in hydrogen gas at about $820^{\circ} \mathrm{C}$. In the next step, which lasts about a second, the heated oxide nanoparticles react with $\mathrm{H}_{2} \mathrm{~S}$ gas at $840^{\circ} \mathrm{C}$ and are sulfidized at the surface. The sulfide encapsulated oxide nanoparticles have passivated surfaces and cannot grow further. Subsequently, in a rather slow diffusion-controlled process, the entire oxide core of the nanoparticles is converted into a metal disulfide in a highly uniform and regular fashion at $840-950^{\circ} \mathrm{C}$. The key element in the success of this strategy is that during this high temperature process the incipient oxide nanoparticles are allowed to interact with the carrier $\left(\mathrm{N}_{2}\right)$ and $\mathrm{H}_{2}$ gases, but their interaction with each other is mostly excluded before they are passivated by the reaction with $\mathrm{H}_{2} \mathrm{~S}$; this leads to the formation of a protective sulfide top layer. Once this protective layer has been formed, the completion of the oxide to sulfide conversion can be done in the bulk, that is, without isolation of the nanoparticles. $\mathrm{WO}_{3}$

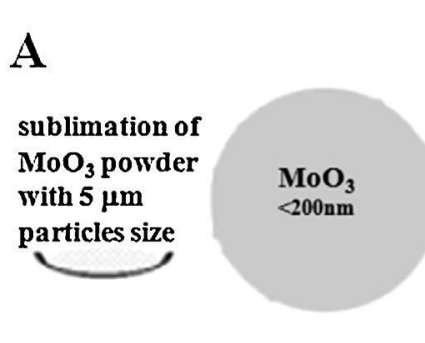

Gas-Phase reaction

nested
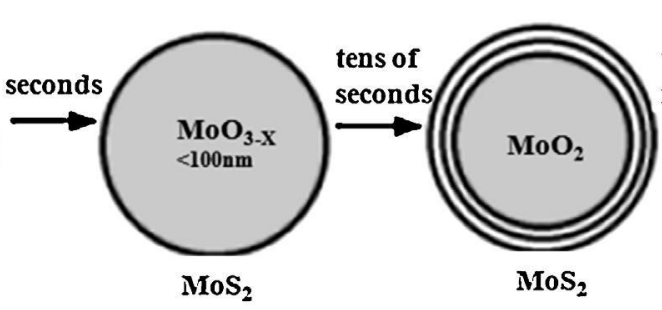

fullerene-like
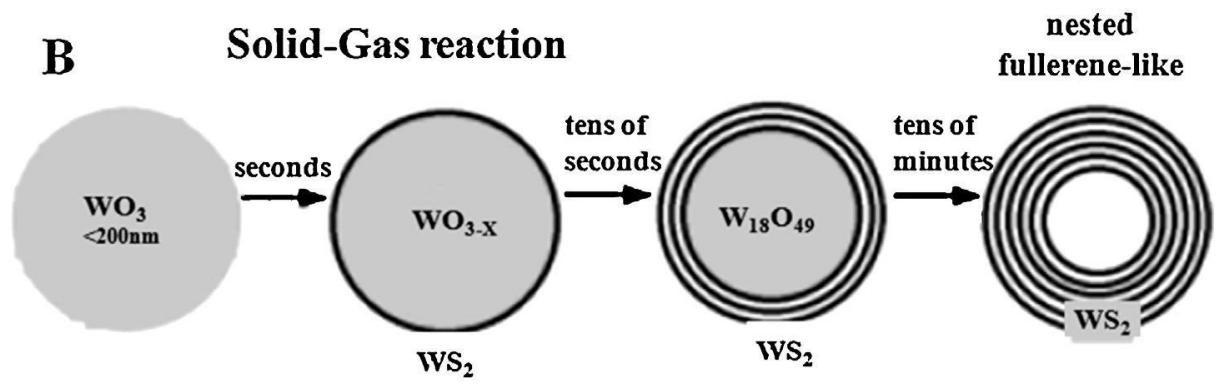

Fig. 7. A schematic drawing of the growth process of fullerene-like $\operatorname{MoS}_{2}\left(\mathrm{WS}_{2}\right)$ from the respective oxides. Reprinted with permission [44]. Copyright 1996, American Chemical Society. 
is much less volatile than $\mathrm{MoO}_{3}$, and hence the precursors for IF-WS 2 , that is, the tungsten oxide nanoparticles, cannot be formed by sublimation. Therefore, a pre-prepared $\mathrm{WO}_{3}$ powder with particle sizes of 0.02-0.2 $\mu \mathrm{m}$ was employed. These small tungsten oxide nanoparticles can be prepared in a number of ways, for example, by heating a tungsten filament to $1600^{\circ} \mathrm{C}$ inside an evacuated bell jar in the presence of water vapor, or by calcination of tungstic acid. Once the details of the reaction mechanism were unraveled, a fluidized-bed reactor for the synthesis of more than $40 \mathrm{~g}$ per day of a pure phase of IF-WS 2 nanoparticles was established [45]. Various synthetic methods for the IF-MS 2 have been reported, such as solid-gas or gas-phase reactions [46], electron beam irradiation activation and arc discharge $[47,48]$, thermal decomposition [49], hydrothermal or solvothermal synthesis [50], the sonochemical process [51], pulsed laser ablation in water [52], etc. However, the most commonly used technique suitable for industrialquantity manufacture is the oxide-to-sulfide conversion using $\mathrm{H}_{2} \mathrm{~S}$ at high temperature under a reducing atmosphere, where the original spherical or rod morphology remains almost unchanged after conversion [27,53]. Using this technique it has been possible to commercialize IFs.

\section{Preparation and dispersion of inorganic (IF) nanoparticles into thermoplastic polymers}

In order to optimize the novel properties of polymer nanocomposites, preparative methods that can control the particle size distribution, dispersion, and interfacial interactions are critical. Synthetic techniques for nanocomposites can be quite different to those used for conventional microscale-filled composites, and the development of a single and universal technique for the preparation of PNCs is impossible due to the wide physicochemical differences encountered in each system. Amongst several factors involved in the selection of an adequate synthetic method, the geometry of the nanofillers is the most prominent and can be generally classified into three categories: (i) one dimensional (1D) nanofillers that have one size dimension (e.g., diameter) $<100 \mathrm{~nm}$ and an aspect ratio $>100$, and include CNTs, (ii) 2D plate-like nanofillers, typically defined as layered materials with a thickness of the order of $1 \mathrm{~nm}$ and an aspect ratio in the other two dimensions of at least $25 \mathrm{~nm}$, such as layered silicates or graphenes, and (iii) 3D (or OD) nanoparticles, which are equiaxial particles with $<100 \mathrm{~nm}$ in their largest dimension, such as nanosilica or fullerenes. Here only the most commonly employed preparation procedures for inorganic polymer nanocomposites (I-PNCs) based on 3D nanoparticles are considered, and can be classified into three broad groups: (i) direct mixing or blending of the polymer and the nanofiller either as discrete phases (dry mixing or melt mixing) or in solution (solution mixing), (ii) sol-gel processes, and (iii) in situ grafting polymerization of macromolecular chains on the surface of nanoparticles. For a summary of synthetic methods used for the processing of PNCs reinforced with different types of inorganic nanofillers the reader is referred to the following publications [7,14,54].

Most direct mixing methods are based on liquid particle dispersions, but differ in the type of the continuous phase (Fig. 8a). In melt processing, particles are dispersed into a polymer melt and then the PNCs are obtained using extrusion and compression or injection molding techniques. However, the melt mixing method also has several drawbacks. Firstly, only relatively weak interaction forces are generated between the polymer and the nanoparticles. The nanoparticles have a very strong tendency to aggregate and the most efficient dispersion is only successfully achieved after surface modification of the inorganic nanoparticles. Secondly, for some polymers this processing method may be limited due to rapid increase of the viscosity with the addition of even very low nanoparticle volume fractions. Some of the limitations of melt mixing can be overcome if both the polymer and the nanoparticles are dissolved or dispersed in solution. This allows modification of the particle surface without drying, reducing particle agglomeration. After dispersion, the polymer nanoparticle solution can then be cast into a solid, or be obtained from solution by solvent evaporation or precipitation [3,7]. Further processing can then be undertaken using conventional techniques.

The sol-gel process starts with a mononuclear species that forms metal oxide frameworks by hydrolysis and condensation reactions (Fig. 8b) [54,55]. Molecular species such as certain organometallic compounds based on alkoxides of silicon, titanium, tin, aluminum, or zirconium act as precursors for this class of hybrids. Frequently used strategies for applying the sol-gel approach to the formation of hybrid materials are (i) mixing the precursor for the sol-gel process with the organic polymer followed by an inorganic polycondensation reaction, (ii) polymerization of an organic monomer in the inorganic gel, (iii) simultaneous formation of the inorganic and organic network, and (iv) dual-network materials in which the two networks are covalently linked to each other. In the conventional sol-gel route, the inorganic components that are generally polydisperse and locally heterogeneous in chemical composition, generate amorphous materials exhibiting a multitude of microstructures [56]. This strategy is simple and inexpensive, but the loss of volatile by-products formed in the hydrolysis and condensation reactions makes it difficult to control sample shrinkage during processing. Organopolysiloxane materials are the most important group of hybrids prepared by sol-gel methods. Tetraethoxysilane, tetramethoxysilane, functional silane coupling reagents such as 3-glycidyloxypropyltrimethoxysilane (GPTMS), 3methacryloxypropyltrimethoxysilane, KH-570, and monomers that contain an organic bridging group and two or more trialkoxysilyl groups have been used extensively in the sol-gel process [7,14,54].

Another method for reducing phase separation is graft polymerization where the nanoparticles are dispersed in the monomer or monomer solution, and the resulting mixture is polymerized by standard polymerization methods. Besides tailoring specific properties in the composites via relatively strong interactions, the layer of the polymer bonded to the nanoparticles can minimize nanoparticle aggregation. In particular, there are two approaches (Fig. 8c) for graft polymerization in the literature. One approach is "grafting-to" (also termed "tethering") that 
(a)

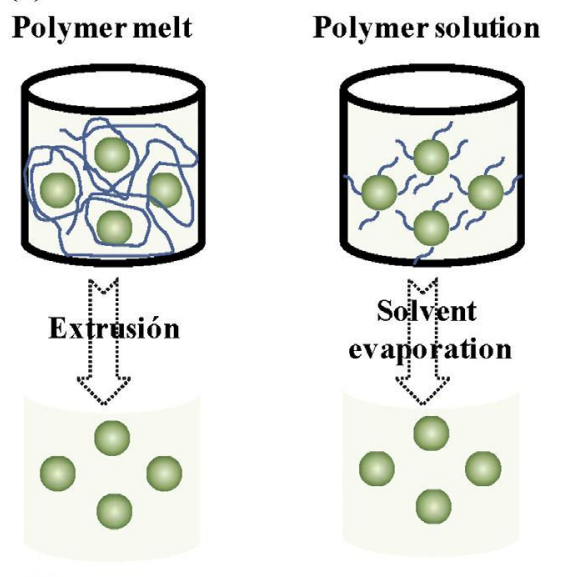

Nanoparticles
Hydrolysis

$$
\mathrm{M}(\mathrm{OR})_{\mathrm{n}} \stackrel{\mathrm{H}_{2} \mathrm{O}}{\longrightarrow} \mathrm{M}(\mathrm{OR})_{n \cdot m}(\mathrm{OH})_{m}+\mathrm{HOR}
$$

\section{Condensation}

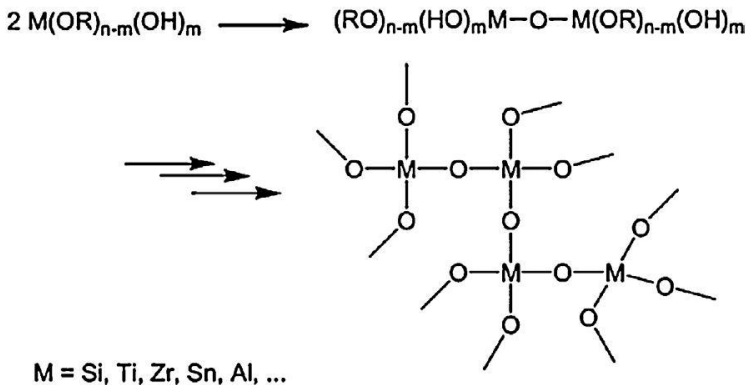

$\mathrm{R}=\mathrm{Me}, \mathrm{Et},{ }^{\mathrm{Pr}},{ }^{\mathrm{P}} \mathrm{Pr},{ }^{n} \mathrm{Bu},{ }^{\mathrm{s}} \mathrm{Bu}, \ldots$

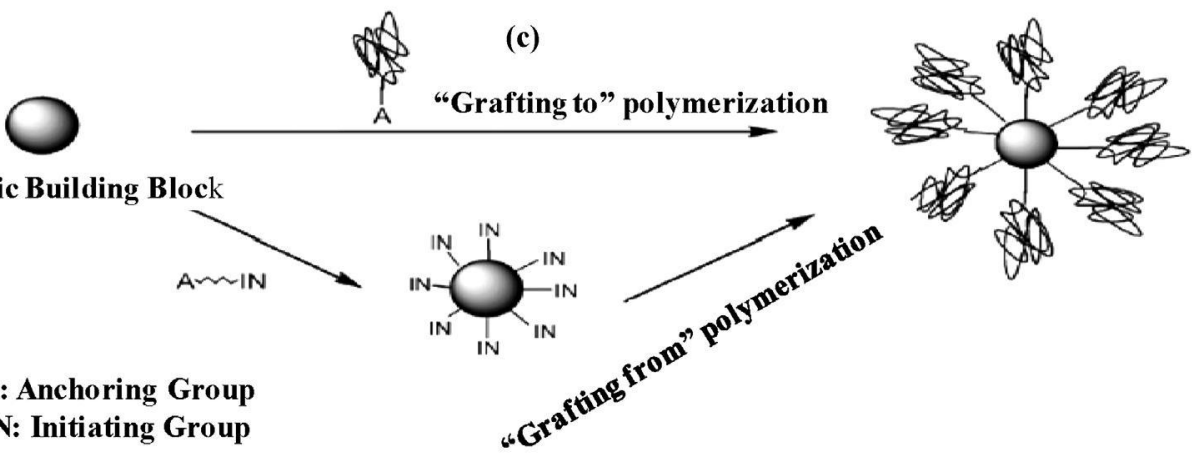

Fig. 8. Common methods used for the preparation of polymer inorganic composite (PINC) materials: (a) direct mixing, (b) sol-gel process and (c) in situ polymerization.

(a,b) Reprinted with permission [54]. Copyright 2003, Elsevier.

consists in the immobilization of polymerizable groups on the nanoparticle surface for copolymerization. The other is the "grafting-from" approach (also known as surfaceinitiated polymerization) that involves grafting an initiator onto the nanoparticle surface to grow the polymer chains from the surface. The grafting-to approach has been mainly conducted via two routes: (i) monomers are copolymerized from the active compounds covalently attached to the inorganic particle surface. Surfactants such as silane coupling agents, polyhedral oligomeric silsesquioxanes (POSS) and octasilsesquioxane $\left(\mathrm{R}_{8} \mathrm{Si}_{8} \mathrm{O}_{12}\right)$ nanoclusters are used for modification of the particle surface to introduce polymerizable groups, and (ii) ready-made polymers with reactive ends react with the functional groups of different monomers pre-grafting onto the nanoparticle surface through physical treatment such as irradiation [54]. Similarly, the grafting-from approach has attracted considerable attention in the preparation of graft polymers on a nanoparticle surface. In the first step, the particle is modified with initiator-bearing self-assembled monolayers. These monolayers can be formed on almost any surface, as long as the anchor functionality is correctly chosen. Next, the nanoparticle surfaces with grafted initiator are exposed to solutions containing a catalyst and monomer. This polymerization is both surface-initiated and surfaceconfined. In particular, this field has rapidly developed over the last decades and many controlled polymerization strategies have been used such as living anionic surfaceinitiated polymerization (LASIP), living cationic surfaceinitiated polymerization (LCSIP), living free radical surfaceinitiated polymerization (LFRSIP), atom transfer radical surface-initiated polymerization (ATRSIP), living ring opening polymerization (LROP), ring-opening metathesis polymerization (ROMP), group transfer polymerization (GTP), and hyper-branched polymerization, etc. [7,14,54].

A smaller particle size allows a much more homogeneous distribution and can lead to a drastic increase of the polymer-nanoparticle interfacial area due to the high SSA of the nanoparticles. However, this may also induce aggregation of the nanoparticles, thus lowering the homogeneity of the particle distribution. In order to minimize interfacial energies between the particles and the polymer matrix, several surface modification or functionalization and stabilization techniques have been developed that are principally used in chemical methods (e.g., sol-gel, in situ polymerization).

Recent work with IF nanoparticles have raised new expectations, since it has been observed in a number of 
polymer systems that these nanoparticles can be efficiently dispersed in the polymer matrix employing traditional methods. In the case of thermoplastic polymer/IF-WS 2 nanocomposites several formulations have been developed that have shown promise for breaking the performance ceiling of current PNCs in terms of thermal, mechanical and tribological properties [57,58], and were prepared using the most simple, cost-effective and ecologically friendly melt processing techniques. These materials can be grouped into three broad categories: IF-PNCs based on commodity polymers, IF-PNCs based on engineering polymers, and IF-PNCs based on high performance thermoplastics that will be considered in the following sections.

Although the discussion will be centered on thermoplastic PNCs, it is important to mention the application of IFs as nanofillers in thermosetting resins. Indeed the first PNCs using these inorganic nanoparticles were developed by Rapoport et al. [59] based on epoxy resins and IF-WS 2 where the beneficial role of IFs for the improvement of the tribological properties were demonstrated. Several subsequent studies showed that IFs could be employed effectively to toughen epoxy resins without sacrificing strength, rigidity and glass transition temperature $\left(T_{g}\right)[60]$, and improve shear strength and fracture behavior [61].

\section{Thermoplastic polymer nanocomposites based on inorganic fullerene-like nanoparticles (IF-PNCs)}

In this section the morphology, thermal stability, crystallization behavior, thermal conductivity, mechanical and tribological properties of the main types of thermoplastic polymer nanocomposites based on IF nanoparticles (IF-PNCs) is described, and their properties compared with those of nanocomposites based on other spherical or pseudo-spherical inorganic nanoparticles as a function of the nature of the thermoplastic polymer matrix. Detailed studies that consider processing, characterization and performance evaluation are important for insight into the structure-property-performance relationships of such nanocomposites. In this respect, a recent review provided an extensive overview for the case of PEKbased nanocomposites reinforced with carbon nanofillers, including carbon nanotubes and nanofibers, and inorganic nanoparticles, including metal oxides and fullerene-like nanoparticles [62], some of which will be commented here.

\subsection{Morphology}

Nanoparticles have a much higher surface-to-volume ratio than conventional micron-sized particles, and with decreasing particle size the number of molecules/atoms present on the surface increases enormously [63]. As a consequence, interparticle attraction through forces such as van der Waals and electrostatic forces, as well as magnetic attraction, becomes stronger resulting in a high surface energy and, as mentioned previously, a tendency to agglomerate during melt blending [64-66]. As already commented, strategies for the preparation of I-PNCs include mechanical blending [67,68], solution mixing [69-75], in situ polymerization [76-85] and in situ nanoparticle synthesis [86-88]. However, guest-host incompatibilities that can be introduced using these approaches frequently lead to nanoparticle aggregation and phase separation over relatively large length scales [89-91]. Without adequate treatment to reduce the surface energy it is very common for clusters or agglomerates to form that are difficult to disperse uniformly in the polymer matrix [92]. Coating the nanoparticle with a thin layer of polymer and surface functionalization of the nanoparticles can reduce or remove influence of van der Waals forces and the compatibility between the organic polymer host and inorganic nanoparticles can be improved, leading to more efficient nanoparticle dispersion and increased effective loading amount.

In some cases the adsorption of polymer chains on the particle surface is considered essential for polymer-filler interaction. Silica is a widely used nanofiller with a large number of hydroxyl groups on its surface that generate strong filler-filler interactions and adsorption of polar materials through hydrogen bonding. However, in the case of apolar polymers such as polypropylene (PP) the adhesion between hydrophilic filler and hydrophobic matrix must be enhanced in some way. Various routes have been proposed, such as pre-treatment of the surface of the filler using active agents, or the addition of hydrophilic coupling agents, and modification by grafting to induce a compatibilizing role affecting the dispersion of filler particles in the composite.

\subsubsection{Polypropylene}

PP is one of the most versatile commercial thermoplastic materials due to its high availability in the market, low cost and balanced properties. To improve certain physical properties of PP in order to meet the requirements of specific applications a wide number of nanofillers have been dispersed within this thermoplastic polymer.

Fig. 9 shows a SEM image of the surface of a cryogenically fractured isotactic polypropylene (iPP) nanocomposites with 4 wt.\% IFWS $_{2}$ prepared from the melt at $210^{\circ} \mathrm{C}$ under optimum processing conditions [93]. The upper image shows homogeneous nanoparticle dispersion (bright spots) where the signals for $\mathrm{C}$ in the matrix and $\mathrm{W}$ and $\mathrm{S}$ in the nanofiller were verified by EDX microanalysis. In the lower image it can be seen that these nanoparticles are almost spherical with an average diameter of around $80 \mathrm{~nm}$, similar to that observed for the neat nanofiller.

It has been demonstrated by statistical analysis of the surface density of IF-WS 2 nanoparticles in this type of nanocomposites that the degree of dispersion strongly depends on the duration of melt blending, Fig. 10. At concentrations of around $8 \mathrm{wt} . \%$ the inter-particle distance is very small and flocculation may take place forming aggregates of between 2 and 5 nanoparticles.

Vassiliou et al. [94] found $\mathrm{SiO}_{2}$ aggregates in iPP composites with fumed silica $12 \mathrm{~nm}$ nanoparticles at concentrations between 2.5 and $10 \mathrm{wt} \%$, distributed randomly in the polymer matrix with an aggregate size that increased with the concentration of nanofiller. Kazuo Asuka et al. [95] described the homogeneous dispersion of totally surface hydroxylated silica powder in iPP by melt processing, Fig. 11 a, for a composition of $5 \mathrm{wt} \% \mathrm{SiO}_{2}$. Very recently, Zhang et al. [96] described the uniform and segregated dispersion of $20 \mathrm{~nm} \mathrm{SiO} 2$ and $50 \mathrm{~nm} \mathrm{CaCO}_{3}$ nanoparticles in 

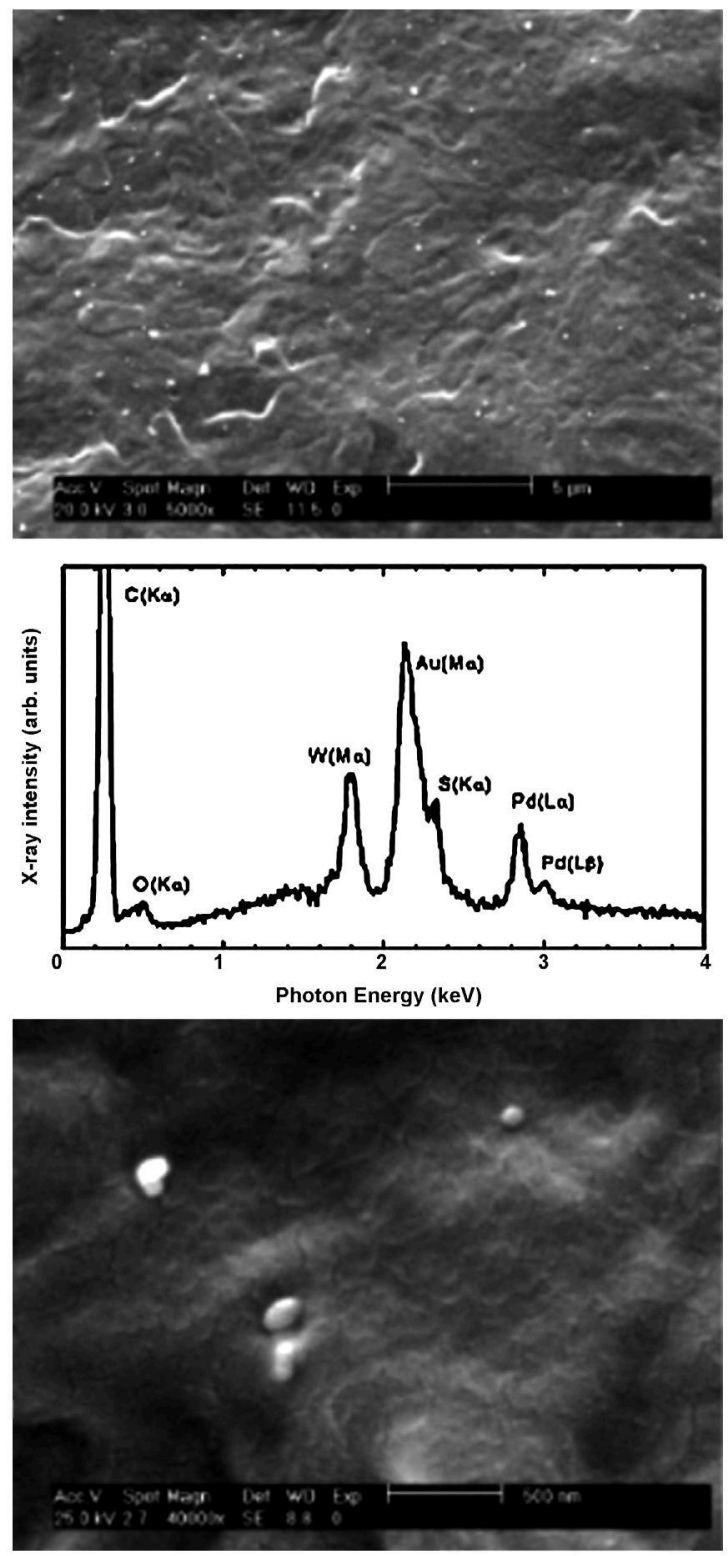

Fig. 9. Top and bottom panels: SEM micrographs at different magnifications from a fractured surface of iPP/IF-WS 2 (4wt.\%) nanocomposite metallized with a $\sim 5 \mathrm{~nm} \mathrm{Au} / \mathrm{Pd}$ overlayer. Middle panel: EDX spectra measured at the particle location.

Reprinted with permission [93]. Copyright 2007, John Wiley and Sons.

ternary nanocomposites with iPP prepared by melt blending.

Kruenate et al. [97] prepared PP nanocomposites in the presence of a modified polyacrylate as dispersing agent after previously modifying the surface of $\mathrm{SiO}_{2}$ nanoparticles obtained by a sol-gel process with a thiocyanatopropyltriethoxysilane coupling agent. The use of polyacrylate slightly reduced the size of the $\mathrm{SiO}_{2}$ agglomerates as a result of the steric stabilization of the dispersing agent adsorbed on the nanofiller surface, Fig. 11b. Papageorgiou et al. [98], using a dichlorodimethylsilane (DCMS) coupling agent with $12 \mathrm{~nm} \mathrm{SiO}_{2}$ nanoparticles, found an

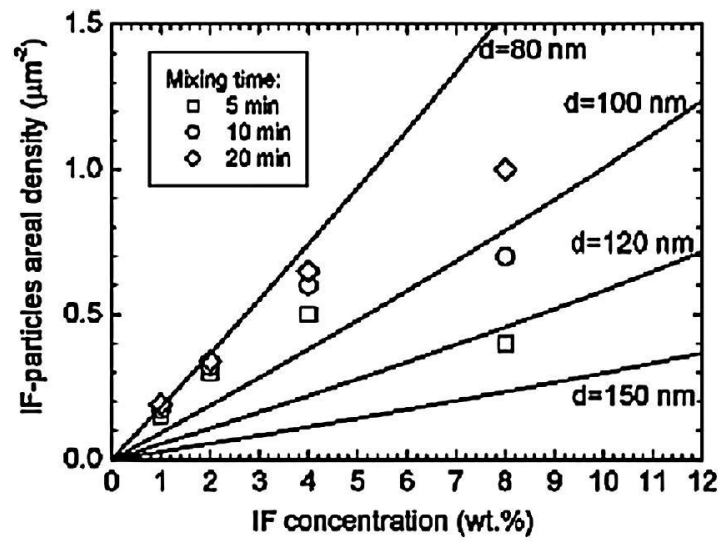

(a) $1 \mathrm{wt} \%$ IF-WS

(b) 2 wt $\%$ IF-WS
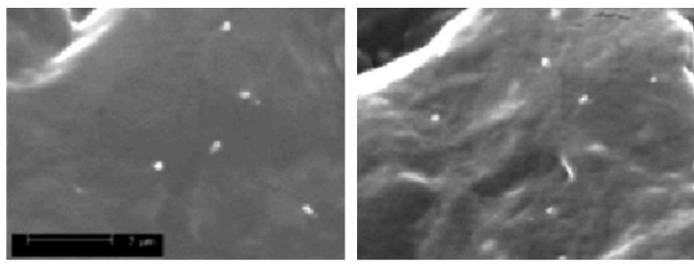

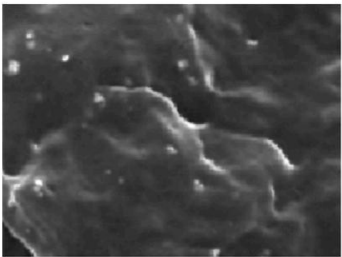

(c) $1 \mathrm{wt} \%$ IF-WS

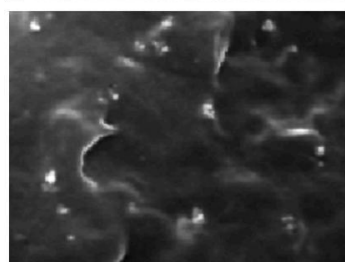

(d) $8 \mathrm{wt} \%$ IF-WS 2
Fig. 10. Bottom panel: SEM micrographs from fractured surfaces of nanocomposites with IF-WS 2 contents of 1,2,4 and $8 \mathrm{wt} \%$ prepared with $10 \mathrm{~min}$ mixing time. Top panel: Experimental values of the number of particles per unit area for nanocomposites with different IF-WS $\mathrm{W}_{2}$ contents and mixing times (dots) and model curves for different particle diameters (lines).

Reprinted with permission. Copyright 2007, John Wiley and Sons [93].

elevated number of nanoparticles in the formed aggregates that reached dimensions of up to $500 \mathrm{~nm}$ at a $10 \mathrm{wt} . \%$ filler concentration. Nitta et al. [99] studied nanocomposites of PP of varying molecular weight and molecular weight distribution with different nanosilicas that were surface modified with coupling agents, Fig. 11c. Chen et al. [100] very recently incorporated the antioxidant methyl 3-(3,5-di-tert-butyl-4-hydroxyphenyl) propionate to the silica surface via transesterification, notably reducing nanoparticle aggregation. Also, ionic liquids such as 1-decyl-3-methylimidazolium tetrafluorborate have been used in the preparation of silica-ionic liquid hybrid aerogels using a sol-gel process, for the subsequent preparation from the melt of nanocomposites with PP [101].

The use of compatibilizing agents that incorporate molecular segments with certain miscibility with the hydrophobic iPP have been widely employed [102-106]. Recently, Palza et al. [104-106] incorporated spherical nanoparticles, synthesized with sol-gel technology through a "wastage mixed semibatch" method [107], into a PP matrix using maleic anhydride-grafted polypropylene 

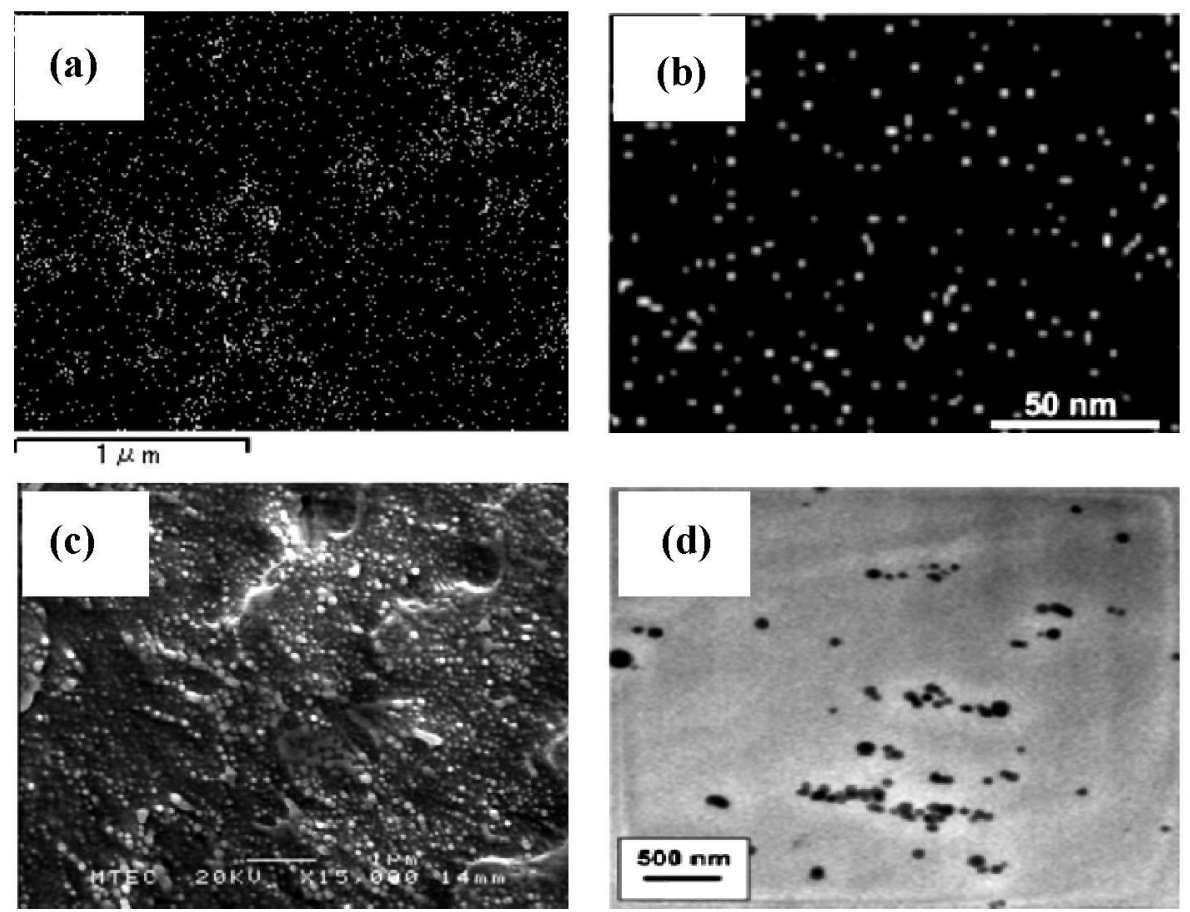

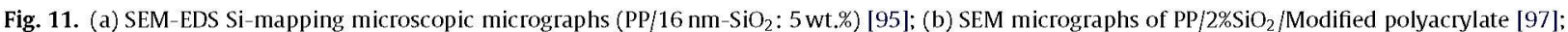
(c) Scanning electron micrograph (SEM-EDS) Si-mapping in iPP composites filled with silica particles having the size of 16 nm [99]; (d) HRTEM images of the nanocomposite with 5 wt.\% of nanofiller dispersed in the PP matrix using PP-g-MA as compatibilizer [106].

Reprinted with permission [95]. Copyright 2006, John Wiley and Sons; [97] Reprinted with permission, Copyright 2004, John Wiley and Sons; [99] Reprinted with permission. Copyright 2006, Elsevier; [106] Reprinted with permission. Copyright 2010, John Wiley and Sons.

(PP-g-MAH) as a compatibilizing agent. Compared to the initial highly agglomerated state, the nanoparticles were well dispersed, Fig. 11d, due to the previous incorporation of the graft copolymer and its subsequent compatibilization with the hydrophobic PP matrix.

Another widely used route for the modification of the interfacial activity of silica nanoparticles is the grafting of polymer chains to the surface via in situ polymerization in a step previous to or during the formation of the nanocomposites in the melt phase [108-111]. Rong et al. [108] irradiated $\mathrm{SiO}_{2}$ nanoparticles of an average diameter of $7 \mathrm{~nm}$ in the presence of monomers such as styrene, methyl methacrylate, butyl acrylate, methyl acrylic acid and vinyl acetate, and subsequently the grafted nanoparticles were blended with PP. The grafting process has also been undertaken in the presence of peroxide-type initiators such as azo-bis-isobutyronitrile, after the prior introduction of double bonds on the silica surface via reaction of the hydroxyl groups with $\gamma$-methacryloxypropyltrimethoxysilane (KH-570) [110]. An alternative strategy includes physical mixing of the nanoparticles with the monomer and the initiator in a planetary ball mill at room temperature and subsequent addition of iPP [112]. Another method is to change the gr afting conditions. Cai et al. [113] grafted poly(p-vinylphenylsulfonylhydrazide-co-butyl acrylate)] as a foaming agent onto the surface of silica with a view to improve the dispersing effect, through the generation of bubbles.

In some cases PP-g-MAH or PP-methyl-POSS, have been used in the formation of composites with untreated $\mathrm{SiO}_{2}$ nanoparticles [114]. In other cases the prior surface functionalization of the silica nanoparticles via grafting with, for example, poly(glycidyl methacrylate)(PGMA) has been used to induce reactive compatibilization with another polymeric component such as aminated polypropylene (PP-g- $\mathrm{NH}_{2}$ ) during the preparation of the nanocomposites from the melt [115].

In the case of $\mathrm{iPP} / \mathrm{CaCO}_{3}$ nanocomposites, Wan et al. [116] made a comparative study of the morphology of nanoparticles with an average size between 60 and $80 \mathrm{~nm}$ that were surface modified either with an aluminatetype coupling agent or in the presence of a blend of aluminate with stearic acid (SA). They found a relatively improved dispersion in the case of the blend due to the existence of stronger interfacial interactions than in the case of the aluminate, and consequently a more effective compatibilization between the $\mathrm{CaCO}_{3}$ nanoparticles and the hydrophobic iPP matrix. Saturated acids such as SA [117,118] and pimelic [119] have also been used as surface modifiers for $\mathrm{CaCO}_{3}$ nanoparticles. Fig. $12 \mathrm{a}-\mathrm{c}$ shows SEM images of iPP nanocomposites with $\mathrm{CaCO}_{3}$ and SA where the filler and the coupling agent were previously blended in a ultra high-speed mixer where the surface modification takes place [118], and the resulting blend was processed from the melt. In the absence of surface modifier the nanofiller-matrix compatibility is deficient and the nanofiller is poorly dispersed, with micrometric-scale aggregates observed, Fig. 12a. In the presence of SA, but with the pre-mixture prepared at $2000 \mathrm{rpm}$, the coalescence is maintained and although the dispersion improves 

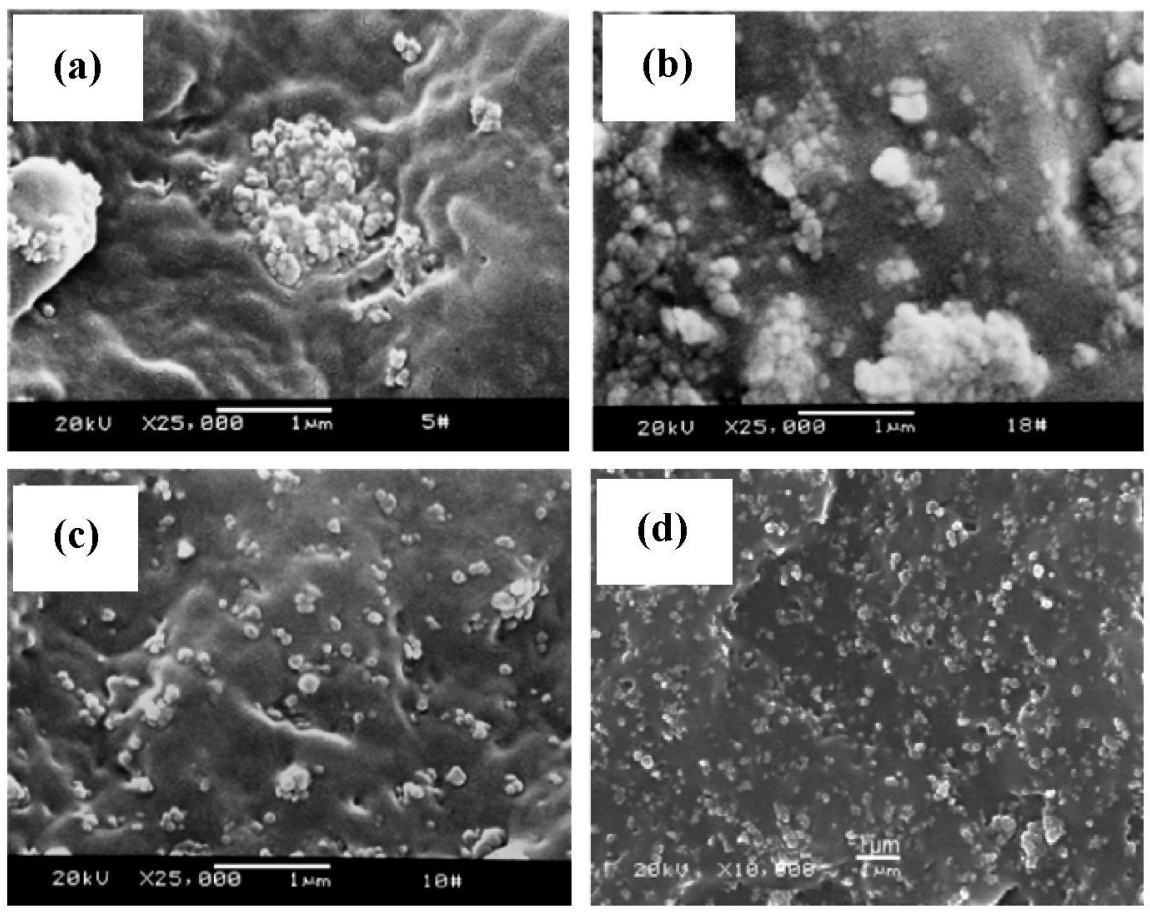

Fig. 12. SEM images of nano- $\mathrm{CaCO}_{3} / \mathrm{PP}$ composites (content of $\mathrm{CaCO}_{3}=11.7$ vol\%). (a) $\mathrm{Nano}^{-\mathrm{CaCO}} 3$ with no treatment. (b) $\mathrm{Nano}_{-} \mathrm{CaCO} 3$ is treated with common high-speed mixer (2000 rpm, $5 \mathrm{~min}$ ). (c) Nano- $\mathrm{CaCO}_{3}$ is treated with ultra-high-speed mixer (6000 rpm, special rotors, $5 \mathrm{~min}$ ) [118]; (d) SEM micrograph of the fracture surface of Izod sample of PP nanocomposites filled with $30 \mathrm{wt} . \% \mathrm{CaCO}_{3}[122]$.

Reprinted with permission [118]. Copyright 2002, Springer; [122] Reprinted with permission. Copyright 2006, John Wiley and Sons.

it is still limited by the formation of aggregates, Fig. $12 \mathrm{~b}$. Only at high speeds, $6000 \mathrm{rpm}$, do the agglomerates seem to disappear and the dispersion become homogeneous with nanoparticles of around $70 \mathrm{~nm}$, even at concentrations as high as $11.7 \mathrm{wt} \%$, Fig. 12c. A good dispersion has been obtained using combinations of SA and hydroxyl propyl methyl cellulose (HPMC) as dispersing media [120,121].

Relatively good dispersions of $\mathrm{CaCO}_{3}$ have been observed even at very high concentrations $[122,123]$. Fig. 12d shows the SEM image of the fracture surface of a iPP composite with $30 \mathrm{wt} \% \mathrm{CaCO}_{3}$ (average particle size $70 \mathrm{~nm}$ ) that was previously treated with $1 \%$ of $\gamma$-aminopropyltriethoxysilane (APTES) coupling agent processed from the melt.

Nanoparticles of $\mathrm{Si}_{3} \mathrm{~N}_{4}$ show very poor compatibility with apolar polymer matrices such as that of iPP. Further, since the diameter of the granules is very small they present a very high tendency to agglomerate. Xu et al. [124] surface treated nanoparticles of 60 and $80 \mathrm{~nm}$ with a $\gamma$ glycidyl propyl trimethoxyl silicane (KH-560) and prepared a nanocomposite with iPP from the melt. In iPP composites with 100 and $300 \mathrm{~nm}$ spherical $C_{3} \mathrm{~N}_{4}$ nanoparticles ( $1 \mathrm{wt} . \%$ ) synthesized using a solvothermal method [125] and prepared from the melt using a microextruder [126], as a result of the shear forces during extrusion the nanoparticles disperse as small clusters without the need to use modifiers or surfactants.

In the case of $\mathrm{ZnO}$ nanoparticles, surface pre-treatment with coupling agents such as thiocyanatopropyltriethoxysilane along with a polyacrylate modified dispersing is also required before melt processing [97]. Tang et al.
[127] analyzed the influence of $\mathrm{ZnO}$ nanoparticles coated with a nucleating agent (NA) on the morphology of iPP, and Zhao and Li [128] studied the use of ZnO as a UV absorber in iPP using coupling agents. Also Huang et al. [129] used dispersing agents to improve the dispersion in iPP nanocomposites prepared by microinjection molding. Lepot et al. [130] have found a good dispersion of spherical $\mathrm{ZnO}$ nanoparticles with initial dimensions of $50-70 \mathrm{~nm}$ in iPP nanocomposites prepared from the melt in the absence of compatibilizing or coupling agents. In other cases, the use of dispersing agents such as SA is not sufficient to avoid nanoparticle coalescence, especially at high ZnO content [131].

Nanocomposites based on iPP and $\mathrm{TiO}_{2}$ nanoparticles have been prepared by melt blending in the presence of dispersing agents such as trisilanol isobutyl polyhedral oligomeric silsesquioxane (POSS) [132]. This type of agent are box-like nanostructured organo-inorganic molecules with a diameter that oscillates between 1 and $3 \mathrm{~nm}$ depending on the composition, and contribute to the dispersion of the nanoparticles since the silanol groups, located on one side, can interact with the polar $\mathrm{TiO}_{2}$ nanoparticles, while the apolar groups interact through van der Waals forces with iPP [133]. Nanocomposites of iPP with $\mathrm{Al}_{2} \mathrm{O}_{3}$ nanoparticles also require surface pre-treatment with silane coupling agents [134].

Nanocomposites of iPP with fullerene- $C_{60}$ have been prepared by surface radical polymerization either through the generation of polymeric precursors employing a radical living polymerization and subsequent reaction with $C_{60}$ [135-137], or by radical copolymerization of $C_{60}$ with 

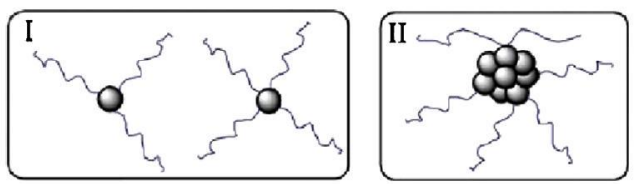

Fig. 13. Schematic drawing for two kinds of long chain branched structures.

Reprinted with permission [143]. Copyright 2011, Royal Society of Chemistry.

a vinyl monomer [138-140]. Martínez et al. [141,142] generated a covalent link between $C_{60}$ and iPP via a direct radical reaction in the presence of a peroxide such as DCP in 1,2,4-trichlorobenzene. Very recently an in situ interfacial interaction between $C_{60}$ and $\mathrm{IPP}$ in the presence of 2,5dimethyl-2,5(tert-butylperoxy)hexane peroxide (DHBP) during melt processing has been described [143], where the average size reduction of $\mathrm{C}_{60}$ aggregates increases with the peroxide concentration. The resulting $P P / C_{60}$ nanocomposites include two types of $C_{60}$ products with grafted $P P$, as can be observed in Fig. 13. One of these includes at least three grafted PP chains on a $\mathrm{C}_{60}$ particle, and the other represents a $C_{60}$ nanoparticle aggregation state with multiple PP branches.

\subsubsection{Nylon 6}

Nylon-6 (polyamide-6, PA-6) is an engineering semicrystalline thermoplastic positioned between commodity plastics and high-performance polymers in price, properties and processing temperature, with good balance of modulus, strength and chemical resistance. Nanocomposites of PA-6 with $0.1-2$ wt.\% spherical IF$\mathrm{WS}_{2}$ nanoparticles (average diameter $80 \mathrm{~nm}$ ) have been prepared from the melt at $240^{\circ} \mathrm{C}$ using a twin-screw miniextruder at $150 \mathrm{rpm}$ without the presence of dispersing agents [144]. Fig. 14 shows a SEM image of a fracture surface of a 2 wt.\% nanocomposite where particles with an average size of around $170 \mathrm{~nm}$ are well dispersed within the matrix.

F. Yang et al. [84] have described the preparation of nanocomposites of PA-6 with silica through in situ polymerization that requires a catalyst and nanofiller surface

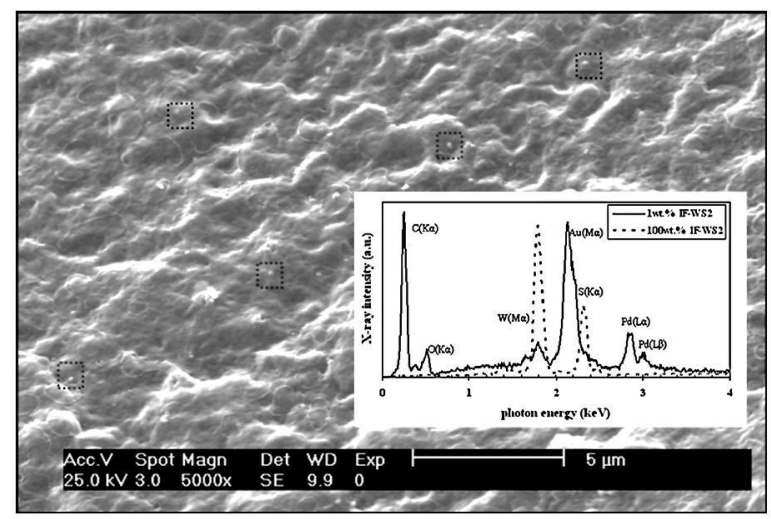

Fig. 14. SEM micrographs of nylon-6/IF-WS 2 (2wt.\%) nanocomposite; inset EDX spectra measured at the particle location.

Reprinted with permission [144]. Copyright 2011, Elsevier.
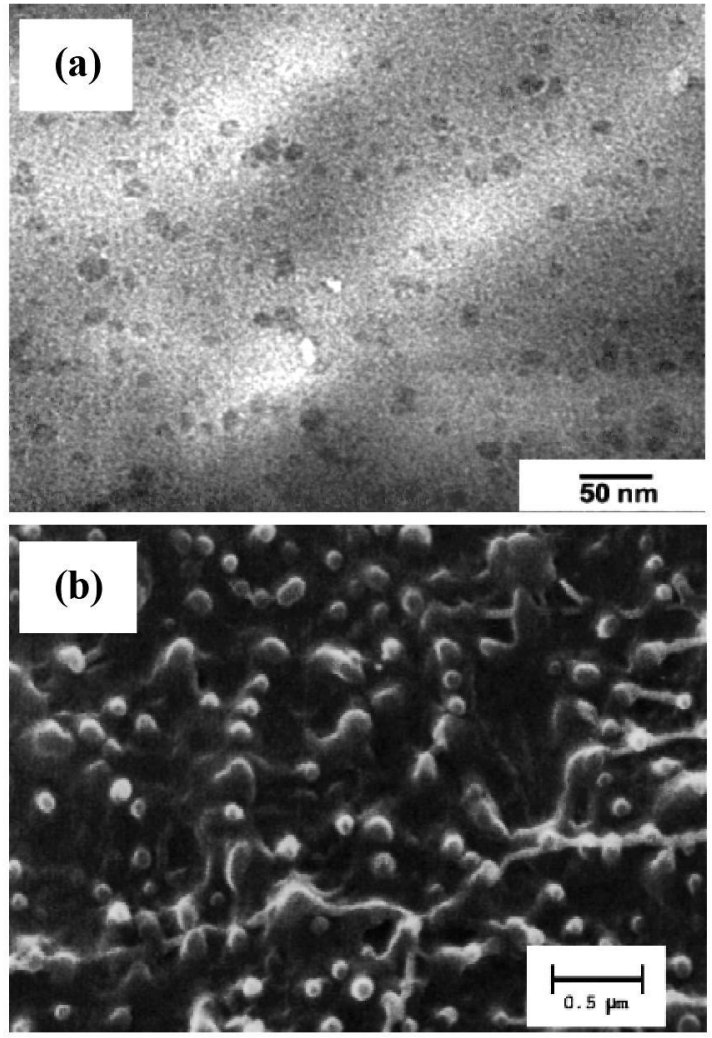

Fig. 15. (a) TEM images of a nylon-6/silica (3\% wt) nanocomposite [79]; (b) Nanocomposite microstructure. The filler weight fraction is 2.5\% [147]. Reprinted with permission [79]. Copyright 2002, John Wiley and Sons; [147] Reprinted with permission. Copyright 2008, Elsevier.

pre-treatment, where the subsequent monomer dispersion and formation of the polyamide takes place by ring opening at high temperature. Because of the high thermal energy of the process a high level of silica aggregation occurs that is especially important when the size of the nanoparticles is less than $50 \mathrm{~nm}$. This methodology is restricted to the family of polyamides formed by cyclic ring opening and not those formed by polycondensation. Van Zyl et al. [79] developed a dissolution-method that, by adequate selection of solvent and $\mathrm{pH}$ in the synthesis, allows the incorporation of $\mathrm{SiO}_{2}$ nanoparticles of between 10 and $30 \mathrm{~nm}$ into nylon 6 . This methodology leads to very low levels of aggregation with the advantage that the formation and separation of the nanocomposites can be undertaken at ambient temperature without nanofiller surface pre-treatment, use of catalyst or initiator, and may be employed for different types of nanofillers, and can be applied to all types of nylon that can be dissolved in formic acid. Fig. 15a shows a TEM image for a Nylon $6 / \mathrm{SiO}_{2}$ (3 wt.\%) nanocomposite.

Later, Shen et al. [145] prepared PA-6/SiO 2 nanocomposites via in situ polymerization using silicic acid as a silica precursor and APTES as coupling agent. Avella et al. [146] surface modified spherical silica nanoparticles of $100-200 \mathrm{~nm}$ size by a grafting reaction in solution with hydroxyl-terminated polycaprolactam (PCL). These grafted nanoparticles were then melt blended with high molecular weight PCL. In Fig. 15b a SEM image of a fracture surface for 
a $2.5 \mathrm{wt} . \%$ nanocomposites shows that the $\mathrm{SiO}_{2}$ nanoparticles are regularly distributed throughout the matrix, and the adhesion between the phases is apparently strong [147].

Rusu and Rusu [148] prepared these nanocomposites by simply dissolving the nanofiller in molten caprolactam by mechanical agitation and found both homogeneous dispersion and the formation of aggregates of up to $200 \mathrm{~nm}$ in size. Recently Cai et al. [149] used ultrasound to disperse $30 \mathrm{~nm}$ average nanosilica in molten PCL. The optimization of the surface coating tended to stabilize both monodisperse nanoparticles and small aggregates against reagglomeration.

\subsubsection{Poly(ether ether ketone)}

Poly(ether ether ketone) (PEEK) is a high-cost highperformance semicrystalline thermoplastic with outstanding mechanical and thermal properties. The incorporation of inorganic nanoparticles into PEEK is primarily aimed at cost reduction and stiffness improvement. However, due to its insolubility in most common solvents and high melting temperature $\left(T_{\mathrm{m}}\right)$, homogenous dispersion of the nanofillers is a difficult task.

The incorporation of IF-WS 2 into PEEK matrices has been achieved with varying degrees of success, as described previously [62]. For example, in the case of an aerosolassisted deposited PEEK/IF-WS 2 nanocomposite coating with $2.5 \mathrm{wt} . \% \mathrm{IF}$, although some agglomeration can be encountered, nanoparticles of $80-220 \mathrm{~nm}$ were observed to be predominatly unidisperse in the matrix throughout the coating thickness, as can be seen in the SEM cross-section image Fig. 16a. In another case where a pre-processing step of ultrasonication in ethanol followed by melt-blending was employed [57], SEM images of the fracture surface of a PEEK/IF-WS 2 ( $1 \mathrm{wt} . \%)$, Fig. 16a, individually dispersed particles or small clusters of 2-3 particles were observed demonstrating the effectiveness of the methodology.

With regard to other inorganic nanoparticles, for quasispherical 20-90 $\mathrm{nm} \mathrm{Al}_{2} \mathrm{O}_{3}$-reinforced $1 \mathrm{wt} . \%$ nanocomposites [150] most nanoparticles remained isolated, with aggregate formations of about $100 \mathrm{~nm}$ observed at higher concentrations up to $10 \mathrm{wt} \%$. At $0.6 \mathrm{vol} \% \mathrm{Al}_{2} \mathrm{O}_{3}$ individual nanoparticles with irregular but almost spherical forms were also observed dispersed within the matrix when prepared by compression molding under vacuum at $400^{\circ} \mathrm{C}$ [151], whereas aggregates of 3-8 nanoparticles could be observed at higher loadings. This is due to the high viscosity of the nanocomposites, and increases with the nanoparticle concentration [152]. In the case of $\mathrm{Si}_{3} \mathrm{~N}_{4}$ nanoparticles a strong tendency to form agglomerates was encountered due to their high specific surface energy [153]. Nonetheless, good dispersion was observed in hot-press processed $\mathrm{PEEK} / \mathrm{Si}_{3} \mathrm{~N}_{4}$ nanocomposites at a nanoparticle concentration of $5 \mathrm{wt} . \%$, when the concentration was $10 \mathrm{wt} . \%$ some aggregation could be observed [153]. Surface treatment of the nanoparticles does not avoid aggregation at high concentrations [154].

In vacuum compression molded $\mathrm{PEEK} / \mathrm{SiO}_{2}$ nanocomposites $[152,155]$ the presence of surface hydroxyl groups increases nanoparticle agglomeration. In this case, the factor that determines phase strength and subequent material
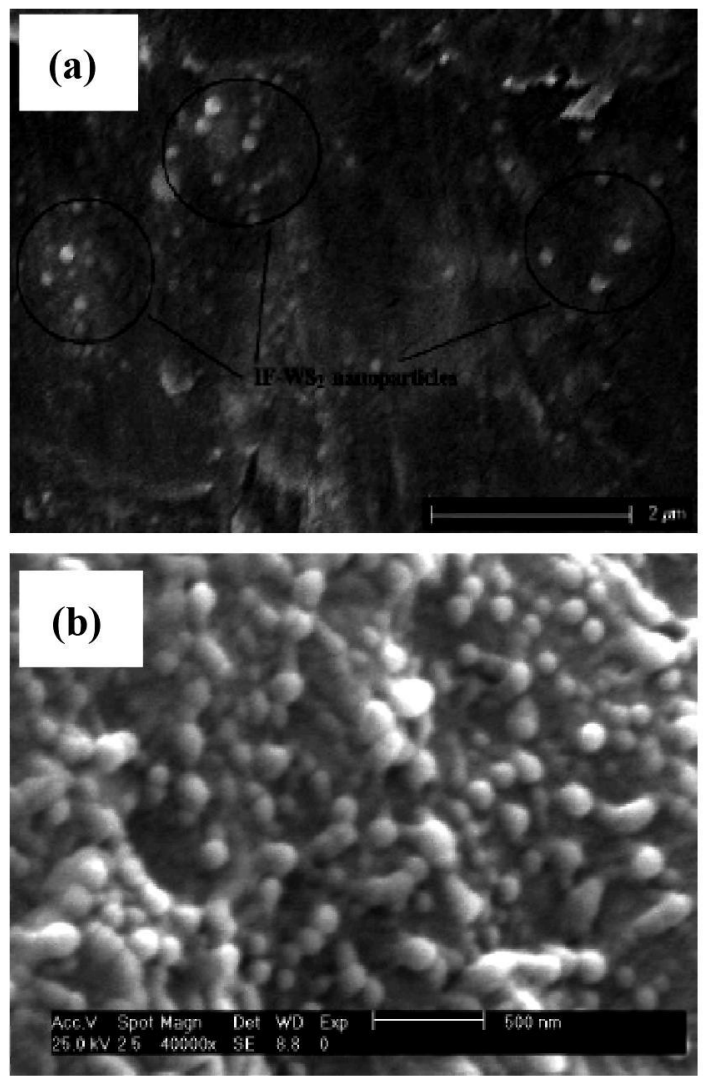

Fig. 16. SEM images of the cross-section of: (a) PEEK nanocomposite coating with 2.5 wt.\% IF-WS 2 nanoparticles [58]; (b) PEEK based nanocomposite with $1.0 \mathrm{wt} \%$ IF-WS 2 [57].

Reprinted with permission [58]. Copyright 2008, Elsevier; [57] Reprinted with permission. Copyright 2010, American Chemical Society.

properties is the interfacial tension between the organic and inorganic phases of the nanocomposite [156-158]. Pre-treatment of the $\mathrm{SiO}_{2}$ nanoparticle surface with SA leads to a reduced aggregate size, particularly at low nanofiller concentrations [159]. Good dispersion and a strong interface was also be obtained by incorporating coupling agents such as silane to the nano- $\mathrm{SiO}_{2}$ replacing hydroxyl groups on the nanoparticle surface that interact with the polymeric matrix [160]. Good dispersions of $\mathrm{SiO}_{2}$ nanoparticles in a PEEK matrix were achieved recently by combining high energy ball milling under argon at room temperature for with surface pre-treatment using a 3-glycidyloxypropyltrimethoxysilane (GPTMS) coupling agent [161].

\subsubsection{Poly(phenylene sulphide)}

Poly(phenylene sulphide) (PPS) is in an engineering thermoplastic widely employed in the electrical, electronics, automotive and chemical sectors due to its excellent chemical, thermal and flame stability, insulating capacity, ageing resistance and moldability. Nanocomposites of PPS with IF-WS 2 have been prepared by melt blending at $320^{\circ} \mathrm{C}$ without nanofiller or polymer pre-treatment [162]. Fig. 17 shows a SEM image of the fracture surface of a PPS/IF$\mathrm{WS}_{2}$ nanocomposite where the bright spots correspond to 

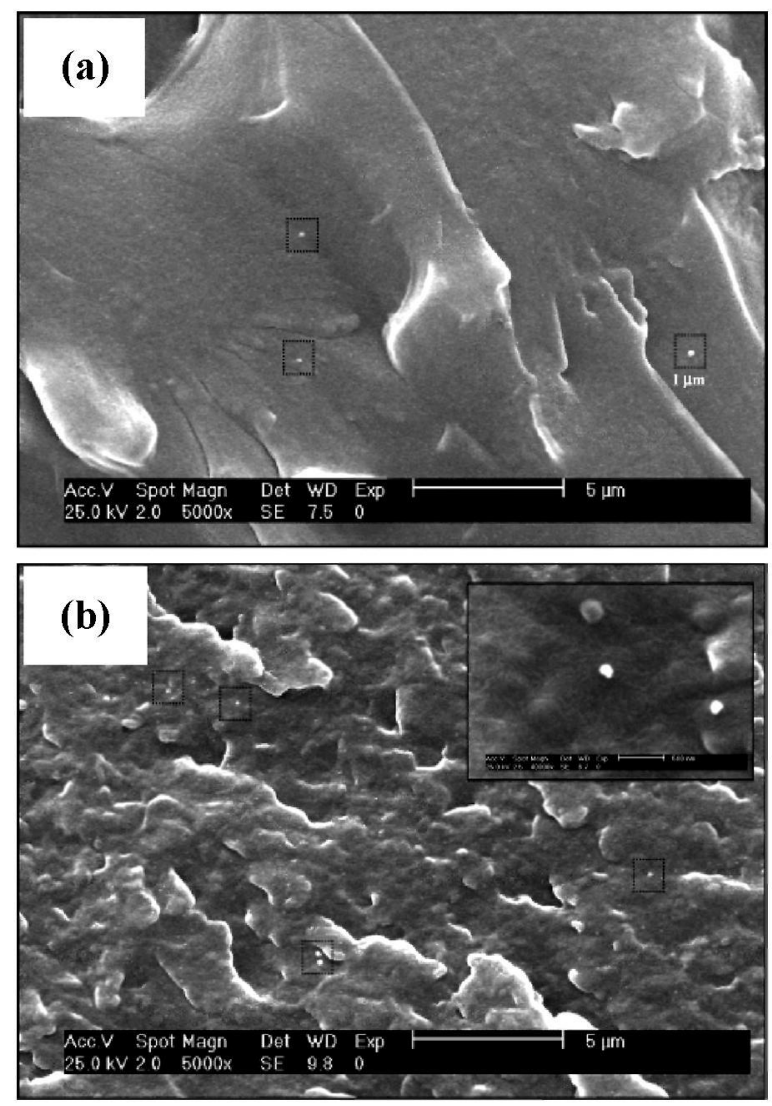

Fig. 17. SEM micrographs of (a) PPS/IF-WS ${ }_{2}(0.1$ wt.\%) and (b) PPS/IF-WS (1 wt.\%) nanocomposites; inset is the SEM micrograph obtained at higher magnification.

Reprinted with permission [162]. Copyright 2008, American Chemical Society.

the sections of small IF nanoparticles aggregates of around $180 \mathrm{~nm}$ [162].

Nanocomposites of PPS have been prepared from the melt with nanofillers such as $\mathrm{SiO}_{x}$, where $x=1.2-1.6$ with sizes between 5 and $15 \mathrm{~nm}$ grafted with epoxy groups using a silane coupling agent [163], and the influence of thermal pre-treatment of PPS and the resulting crosslinking, as well as pre-mixing using ball milling [164] on the dispersion of the nanoparticles and the properties of the nanocomposites has been analyzed.

\subsubsection{Poly (methylene oxide)}

Nanocomposites of polymethylene oxide (POM) with inorganic fullerenes such as IF-MoS $\mathrm{S}_{2}$ have been described by $\mathrm{Hu}$ et al. [165]. These spherical nanoparticles of $100-200 \mathrm{~nm}$ diameter, Fig. 18, were prepared from $\mathrm{Na}_{2} \mathrm{MoO}_{4}$ and $\mathrm{CH}_{3} \mathrm{CSNH}_{2}$ [166] and incorporated into the molten POM matrix. To our knowledge, to date no other PNCs incorporating spherical inorganic nanoparticles have been reported for this matrix.

\subsection{Thermal stability}

In general it is widely accepted that the incorporation of nanometer-sized inorganic particles into a polymer enhances the thermal stability of the matrix inhibiting the formation and escape of volatile by-products generated during the decomposition process. The degradation temperatures observed for the nanocomposites are related to the type, size and concentration of the nanoparticles, their gas impermeability and their interactions with the reactive groups in the polymer. IF-WS 2 nanoparticles display very high thermal stability [93], being stable in nitrogen atmosphere up to temperatures in excess of $800^{\circ} \mathrm{C}$, although in an oxidative environment they start to degrade forming $\mathrm{WO}_{3}$ at $\sim 400^{\circ} \mathrm{C}$, with a small weight loss $\sim 6.5 \%$. Nevertheless, the initial degradation temperature of these particles is usually well above those of commonly used polymers, which will inevitably be the limiting parameter for defining the nanocomposite processing conditions. Under both types of atmosphere, the addition of small amounts of IF-WS $2(\leq 8 \mathrm{wt} . \%)$ to thermoplastic matrices leads to significant increases in their thermal decomposition temperatures, although to date little work is available in the literature $[57,93,167,168]$. Table 3 compares the thermal stability improvements observed via the incorporation of IF-WS 2 , determined by thermogravimetric analysis (TGA), with those attained by the incorporation of other quasi-spherical inorganic nanofillers, by analyzing the difference between the initial degradation temperature of the nanocomposite and that of the neat matrix $\left(\Delta T_{i}\right)$, the variation in the temperatures for 10 and $50 \%$ weight loss $\left(\Delta T_{10}, \Delta T_{50}\right.$ ), and the change in the temperature corresponding to the maximum rate of weight loss $\left(\Delta T_{\mathrm{mr}}\right)$.

\subsubsection{Polypropylene}

The incorporation of IF-WS 2 into iPP was reported to induce a remarkable thermal stabilization of this matrix under both types of atmosphere [93], Fig. 19, and this was more significant at high IF-WS 2 content ( $8 \mathrm{wt} . \%$ ), with increases in $T_{\mathrm{i}}$ of $66^{\circ} \mathrm{C}$ and $38^{\circ} \mathrm{C}$ under inert and oxidative conditions, respectively. Qualitatively similar trends were observed for the other degradation temperatures, where the increments progressively rose with increasing nanoparticle concentration, up to $63^{\circ} \mathrm{C}$ in $T_{\mathrm{mr}}$ under air. The nanocomposites exhibited a single degradation stage analogous to that of the pure matrix in both inert and oxidative atmospheres, indicating that IF-WS 2 does not modify the degradation mechanism of the polymer. In nitrogen a good correlation between the residual mass and the IF-WS concentration was found, suggesting that the carbon atoms from the iPP matrix were released after chain degradation. The degradation was more complex in an oxidative environment due to the formation of carbides, oxides and sulfides that occurred for the composites with 1.0-4 wt.\% loading in the temperature range of $325-500^{\circ} \mathrm{C}$ after the decomposition of more than $80 \%$ of the matrix. Above $500^{\circ} \mathrm{C}$ the TGA curves were flat showing a $\mathrm{WO}_{3}$ residue value that corresponded with the IF-WS ${ }_{2}$ content. It can be highlighted that the improvements in thermal stability attained by adding IF-WS 2 are larger than those reported for PP nanocomposites filled with similar amounts of other unmodified nanofillers such as $\mathrm{SiO}_{2}$ [100], $\mathrm{CaCO}_{3}$ [169] or $\mathrm{ZnO}$ [128]; however, the surface treatment of $\mathrm{SiO}_{2}$ with a silane coupling agent [170] or the use of PP-g-MAH as a compatibilizer [106] led to stronger increments in the degradation temperatures of this thermoplastic, 


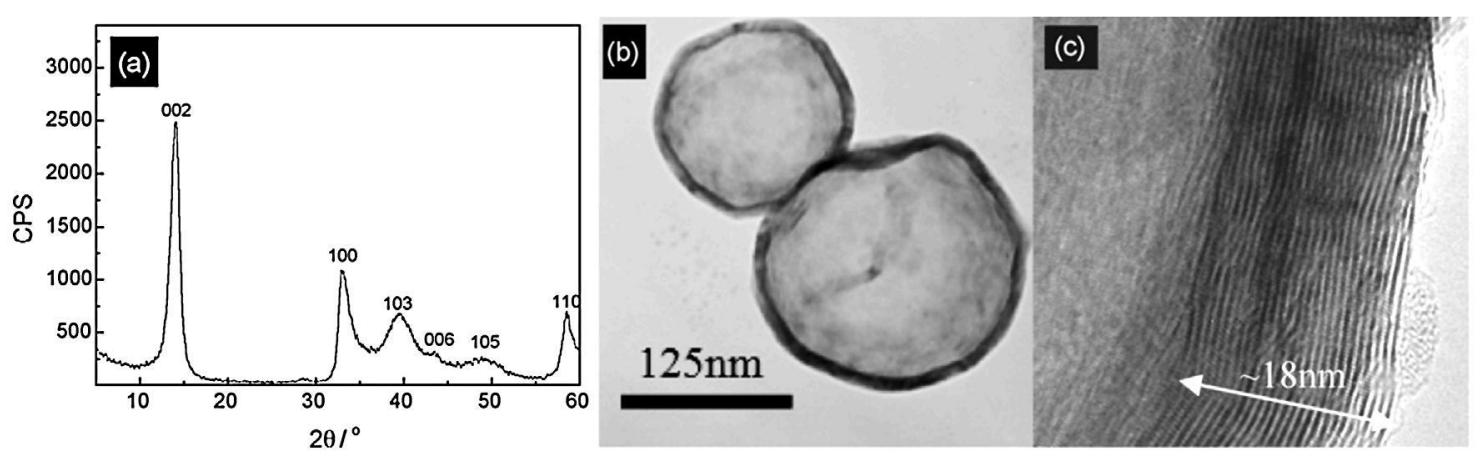

Fig. 18. Results of (a) XRD, (b) TEM and (c) HRTEM for the obtained $\operatorname{MoS}_{2}$ nanoparticles.

Reprinted with permission [165]. Copyright 2009, Elsevier.

associated with improved dispersion of the nanoparticles and stronger nanofiller-matrix interfacial adhesion, and the perturbation of large scale polymer relaxations in the nanocomposite, resulting in reduced permeability of the polymer, and enhanced thermal stability. These nanocomposites [106] exhibited two degradation peaks in oxidative atmosphere associated with different decomposition processes taking place due to the physical/chemical adsorption of the volatile products on the particle surface.

\subsubsection{Nylon-6}

The incorporation of IF-WS 2 has also been demonstrated to be an effective approach to improve the thermal stability of nylon-6 [167].Upon the addition of only $0.1 \mathrm{wt} . \%$
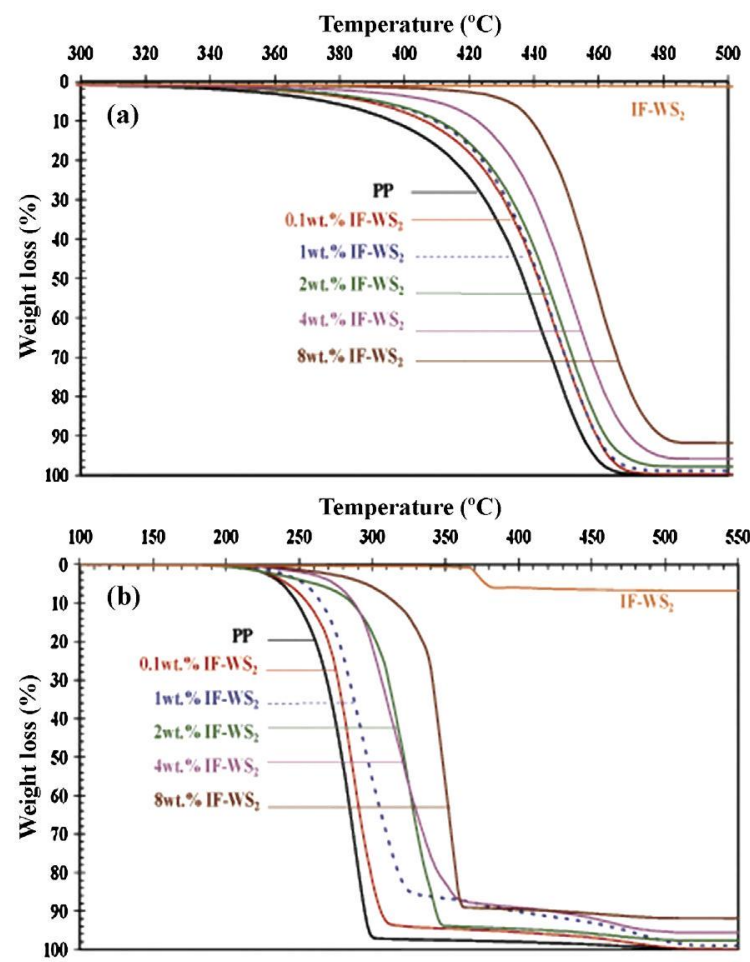

Fig. 19. TGA curves of IF-WS ${ }_{2}$ nanoparticles and iPP/IF-WS 2 nanocom posites under (a) nitrogen and (b) oxygen atmospheres.

Reprinted with permission [93]. Copyright 2007, John Wiley and Sons.
IF-WS $S_{2}$ the value of $T_{\mathrm{i}}$ increased by $\sim 35^{\circ} \mathrm{C}$ under both inert and oxidative atmospheres. However, $T_{\mathrm{i}}$ remained practically unchanged at higher IF-WS 2 contents. Smaller increments were reported for $T_{10}$ and $T_{50}$ under an oxidative environment, in the range of $2-10^{\circ} \mathrm{C}$ for $0.1-2 \mathrm{wt} . \%$ IF$\mathrm{WS}_{2}$, whereas in nitrogen these degradation temperatures were similar to those of the matrix. However, the IF-WS 2 nanoparticles appear to be more efficient for improving the $T_{\mathrm{i}}$ of PA- 6 than similar or even higher amounts of unmodified or surface modified silica [145,171-173]. Opposite to the trend found by adding increasing IF-WS contents, $\mathrm{PA}-6 / \mathrm{SiO}_{2}$ (0.2-2.7 wt.\%) nanocomposites [171] showed a maximum in $\Delta T_{\mathrm{i}}$ at $0.8 \mathrm{wt} . \%$ and at higher loadings the increment in $T_{1}$ decreased considerably. Surface treatment of $\mathrm{SiO}_{2}$ with CA [173] or the addition of a silane coupling agent [145] slightly improved the thermal stability of the resulting nanocomposites, ascribed to stronger nanoparticle-matrix interfacial interactions. An analogous behavior was reported by Rusu and Rusu [174] for $\mathrm{TiO}_{2}$ reinforced nanocomposites, where the incorporation of APTES as a compatibilizer led to larger enhancements in $T_{10}$, attributed to the higher thermal stability of the treated nanoparticles inducing a retarding effect on the thermal degradation of the matrix. Surprisingly, the addition of $6 \mathrm{wt} . \% \mathrm{CaCO}_{3}(70 \mathrm{~nm})$ [175] provoked a noticeable decrease in the thermo-oxidative stability of PA-6, caused by the plasticizing effect of these nanofillers and their agglomerated state, resulting in an acceleration of the matrix degradation process. Conversely, Sonawane et al. [176] reported an extraordinary stability enhancement under inert environment upon incorporation of 1.0-4 wt.\% $\mathrm{CaCO}_{3}(11-23 \mathrm{~nm})$, ascribed to the uniform dispersion and very small nanoparticle size that favor the absorption of heat and prevent the diffusion of volatile decomposition products.

\subsubsection{Poly(phenylene sulphide)}

A study of the thermal stability of PPS/IF-WS ${ }_{2}$ nanocomposites [168] showed that $T_{\mathrm{i}}$ and $T_{10}$ were hardly modified under nitrogen and oxidative environments. In contrast, $T_{50}$ increased significantly at high IF-WS 2 contents, with increments of up to $160^{\circ} \mathrm{C}$ at $2 \mathrm{wt} \%$ loading under inert atmosphere and up to $44^{\circ} \mathrm{C}$ at $8 \mathrm{wt} . \%$ under oxygen. Other studies on nanoparticle-filled PPS are scarce and only notable rises in the degradation temperatures of PPS (up to 
$18^{\circ} \mathrm{C}$ in $T_{\mathrm{i}}$ ) were observed upon the addition of $20-40 \mathrm{wt} . \%$ $\mathrm{Fe}_{3} \mathrm{O}_{4}$ nanoparticles [177], which absorb heat and hinder the transport of degradation products, hence decelerating the decomposition process.

\subsubsection{Poly(ether ether ketone)}

PEEK displays excellent thermal stability, with $T_{\mathrm{i}}$ values at $\sim 480$ and $520^{\circ} \mathrm{C}$ and maximum decomposition rate, $T_{\mathrm{m}}$ values at $\sim 530$ and $555^{\circ} \mathrm{C}$, in oxidative and inert atmospheres, respectively. The addition of increasing IF-WS contents was reported to gradually enhance the stability of this polymer under both types of environments, with increments in $T_{\mathrm{i}}$ of up to $\sim 55^{\circ} \mathrm{C}$ at $2 \mathrm{wt} . \%$ loading [57]. Similar improvements were found for $T_{10}$ and $T_{\mathrm{mr}}$. Hou et al. [58] also reported significant increments $\left(15-25^{\circ} \mathrm{C}\right)$ in the $T_{\mathrm{m}}$ of PEEK/IF-WS $2(1-20 \mathrm{wt} . \%)$ coatings in comparison to that of the pure matrix, ascribed to a strong polymer-nanoparticle interfacial adhesion that hindered the segmental movement of the PEEK molecules. In differential thermal analysis (DTA) curves of the nanocomposites under air atmosphere, no exothermic peak due to degradation of the IF-WS 2 was detected indicating that the nanoparticles were covered and protected by the matrix from oxidation at elevated temperature. When matrix decomposition was initiated the nanoparticles became exposed to the oxidizing environment, and subsequently the oxidation of both coating components occurred simultaneously. It was concluded that the stability of both PEEK and IF-WS $\mathrm{S}_{2}$ was enhanced in the nanocomposite coating. Table 1 clearly shows that the addition of the IF-WS 2 is the most effective method to enhance the thermal stability of PEEK. Under inert atmosphere the increments in $T_{10}$ and $T_{\mathrm{mr}}$ attained at $2 \mathrm{wt} . \% \mathrm{IF}-\mathrm{WS}_{2}$ are larger than those reported for the incorporation of 2.5-10 wt.\% $\mathrm{SiO}_{2}[152,159]$ or $\mathrm{Al}_{2} \mathrm{O}_{3}$ $[150,154]$. In these nanocomposites the degradation temperatures also increased with nanofiller content up to $\sim 10 \mathrm{wt} . \%$ due to the existence of nanoparticle-matrix interactions that restricted the mobility of the PEEK chains. However, at higher loadings the improvements were smaller due to the presence of large nanoparticle agglomerates [150]. The incorporation of SA-treated silica [159] reduced the effect of improvement in the decomposition temperatures in comparison to the unmodified counterparts, since SA displays lower thermal stability than the other components. Goyal et al. [178] also reported significant thermo-oxidative stability improvements in PEEK/AIN (5.2-48 wt.\%) composites in comparison to the neat polymer, albeit the increase found at $31 \mathrm{wt} . \%$ loading $\left(48^{\circ} \mathrm{C}\right.$ ) was the same as the attained upon addition of only $1 \mathrm{wt} . \%$ IF-WS 2 . Balaji et al. [179] described an anomalous behavior for PEEK/Si $\mathrm{N}_{4}$ nanocomposites, where $T_{\mathrm{mr}}$ decreased slightly irrespective of the nanoparticle content. To conclude, the addition of very low IF-WS 2 loading (i.e. $0.1 \mathrm{wt} . \%$ ) to thermoplastic polymers results in almost the same stability enhancement than that attained by the incorporation of $\sim 10 \mathrm{wt} . \%$ of other inorganic nanoparticles.

\subsection{Crystallization behavior}

The crystallization behavior of several polymer matrices where IF-WS 2 nanoparticles have been employed is
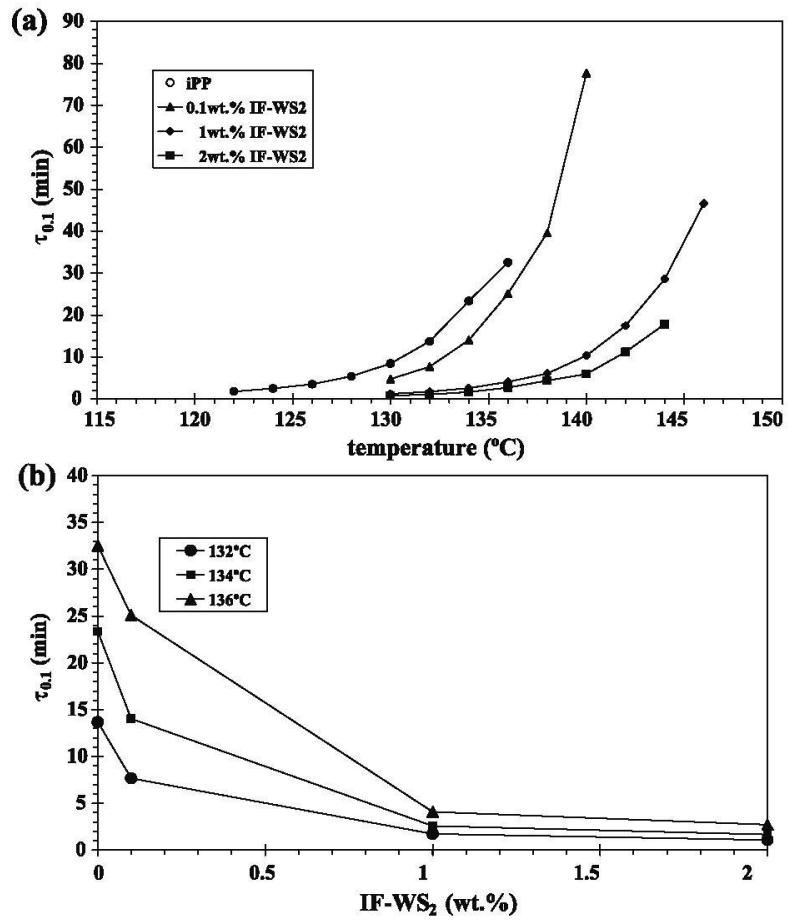

(c)

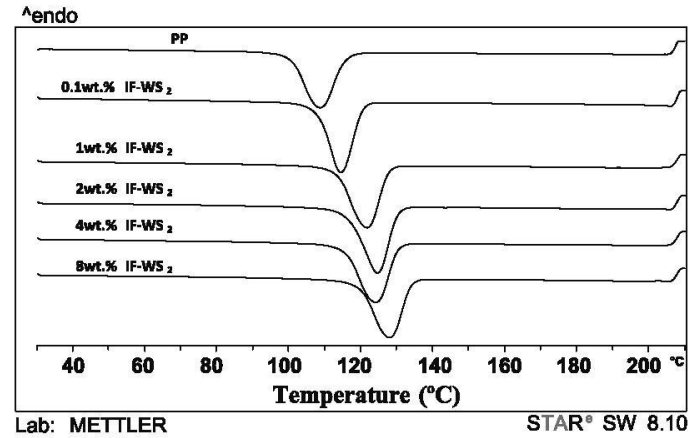

Fig. 20. (a) DSC cooling thermograms of iPP/IF-WS 2 nanocomposites from melt to room temperature at $10^{\circ} \mathrm{C} / \mathrm{min}$ for samples with several IF-WS contents [93]; Time to reach $10 \%$ crystalline transformation $\left(\tau_{0.1}\right)$ for iPP/IF-WS $S_{2}$ nanocomposites as a function of (b) the crystallization temperature and (c) the composition [188].

Reprinted with permission [93]. Copyright 2007, John Wiley and Sons; [188] Reprinted with permission. Copyright 2008, Elsevier.

compared with that observed for other spherical inorganic nanofillers, organized by the nature of the matrix.

\subsubsection{Polypropylene}

Quasi-spherical IF-WS 2 nanoparticles with an average size of $80 \mathrm{~nm}$ act as nucleating agents in both dynamic and isothermal crystallization of iPP [93]. As an example, Fig. 20a shows the DSC thermograms corresponding to $\mathrm{iPP} / \mathrm{IF}-\mathrm{WS}_{2}$ nanocomposites cooled from the molten phase at $210^{\circ} \mathrm{C}$ at a rate of $10^{\circ} \mathrm{C} / \mathrm{min}$ where a shift of the crystallization exotherms towards higher temperature is observed with increasing nanofiller content, and the $\alpha$ or monoclinic polymorph of iPP is maintained over the whole nanofiller composition range independent of the cooling rate. The nucleation efficiency (NE) determined by the Lotz method [180] reaches values of between 60 and $70 \%$, 
Table 1

Thermal stability of thermoplastic/IF-WS 2 and other nanoparticle-filled thermoplastic nanocomposites under inert and oxidative conditions.

\begin{tabular}{|c|c|c|c|c|c|c|c|c|c|c|c|c|}
\hline \multirow[t]{2}{*}{ Matrix } & \multirow{2}{*}{$\begin{array}{l}\text { Nanofiller (Particle } \\
\text { size/nm) }\end{array}$} & \multirow{2}{*}{$\begin{array}{l}\text { Nanofiller content } \\
\text { (wt.\%) }\end{array}$} & \multirow{2}{*}{$\begin{array}{l}\text { Processing } \\
\text { method }\end{array}$} & \multicolumn{4}{|l|}{ Nitrogen } & \multicolumn{5}{|c|}{ Oxygen or air atmosphere } \\
\hline & & & & $\Delta T_{\mathrm{i}}\left({ }^{\circ} \mathrm{C}\right)$ & $\Delta T_{10}\left({ }^{\circ} \mathrm{C}\right)$ & $\Delta T_{50}\left({ }^{\circ} \mathrm{C}\right)$ & $\Delta T_{\mathrm{mr}}\left({ }^{\circ} \mathrm{C}\right)$ & $\Delta T_{\mathrm{i}}\left({ }^{\circ} \mathrm{C}\right)$ & $\Delta T_{10}\left({ }^{\circ} \mathrm{C}\right)$ & $\Delta T_{50}\left({ }^{\circ} \mathrm{C}\right)$ & $\Delta T_{\mathrm{mr}}\left({ }^{\circ} \mathrm{C}\right)$ & Ref. \\
\hline $\mathrm{PP}$ & IF-WS $2(40-80)$ & $\begin{array}{l}0.1 \\
1 \\
2 \\
4 \\
8\end{array}$ & $\mathrm{mb}$ & $\begin{array}{l}13 \\
18 \\
18 \\
33 \\
66\end{array}$ & $\begin{array}{l}11 \\
14 \\
15 \\
27 \\
44\end{array}$ & $\begin{array}{r}4 \\
4 \\
6 \\
12 \\
19\end{array}$ & $\begin{array}{r}5 \\
3 \\
6 \\
10 \\
16\end{array}$ & $\begin{array}{r}1 \\
\mathbf{1 1} \\
3 \\
26 \\
38\end{array}$ & $\begin{array}{r}6 \\
16 \\
36 \\
37 \\
64\end{array}$ & $\begin{array}{r}7 \\
18 \\
42 \\
40 \\
67\end{array}$ & $\begin{array}{r}0 \\
5 \\
38 \\
32 \\
63\end{array}$ & [93] \\
\hline $\mathrm{PP}$ & Fumed $\mathrm{S}_{\mathrm{i}} \mathrm{O}_{2}(12)$ & 5 & $\mathrm{mb}$ & 1 & - & 0 & 1 & - & - & - & - & {$[100]$} \\
\hline PP & $\mathrm{S}_{\mathrm{i}} \mathrm{O}_{2}(50-110)$ & $\begin{array}{l}1 \\
3 \\
5\end{array}$ & $\mathrm{mb}$ & $\begin{array}{r}5 \\
15 \\
13\end{array}$ & $\begin{array}{l}- \\
- \\
-\end{array}$ & $\begin{array}{l}- \\
- \\
-\end{array}$ & $\begin{array}{r}3 \\
11 \\
7\end{array}$ & $\begin{array}{r}0 \\
13 \\
-1\end{array}$ & $\begin{array}{l}- \\
- \\
-\end{array}$ & $\begin{array}{l}- \\
- \\
-\end{array}$ & $\begin{array}{l}42 \\
10 \\
39\end{array}$ & [106] \\
\hline $\mathrm{PP}$ & $\begin{array}{l}\text { PP-g-MAH } \\
\text { modified } \mathrm{S}_{\mathrm{i}} \mathrm{O}_{2} \\
(50-110)\end{array}$ & $\begin{array}{l}1 \\
3 \\
5\end{array}$ & $\mathrm{mb}$ & $\begin{array}{l}39 \\
38\end{array}$ & $\begin{array}{l}- \\
-\end{array}$ & $\begin{array}{l}- \\
-\end{array}$ & $\begin{array}{r}7 \\
14\end{array}$ & $\begin{array}{r}21 \\
0\end{array}$ & - & $\begin{array}{l}- \\
-\end{array}$ & $\begin{array}{l}44 \\
71\end{array}$ & {$[106]$} \\
\hline PP & $\begin{array}{l}\text { APTES-modified } \\
\mathrm{S}_{\mathrm{i}} \mathrm{O}_{2}(7)\end{array}$ & 2 & $\mathrm{dm}+\mathrm{cm}$ & 124 & 110 & 62 & 70 & - & - & - & - & {$[170]$} \\
\hline PP & $\begin{array}{l}\text { AO-modified } \\
\text { Fumed } \mathrm{S}_{1} \mathrm{O}_{2}(12)\end{array}$ & 5 & $\mathrm{mb}$ & 3 & - & 0 & 2 & - & - & - & - & {$[100]$} \\
\hline PP & $\mathrm{CaCO}_{3}(40-70)$ & $\begin{array}{l}1 \\
3\end{array}$ & $\mathrm{mb}+\mathrm{cm}$ & - & $\begin{array}{l}- \\
-\end{array}$ & $\begin{array}{l}- \\
-\end{array}$ & $\begin{array}{l}- \\
-\end{array}$ & - & $\begin{array}{l}- \\
-\end{array}$ & $\begin{array}{l}14 \\
10\end{array}$ & $\begin{array}{l}- \\
-\end{array}$ & [169] \\
\hline PP & $\begin{array}{l}\text { PP-g-MAH } \\
\text { modified } \mathrm{CaCO}_{3} \\
(40-70)\end{array}$ & $\begin{array}{l}1 \\
3\end{array}$ & $\mathrm{mb}+\mathrm{cm}$ & - & - & - & - & - & - & $\begin{array}{l}22 \\
23\end{array}$ & - & [169] \\
\hline $\mathrm{PP}$ & $\mathrm{ZnO}(40)$ & $\begin{array}{l}1.5 \\
3 \\
5\end{array}$ & $m b+i n j$ & $\begin{array}{r}3 \\
14 \\
8\end{array}$ & $\begin{array}{l}- \\
- \\
-\end{array}$ & $\begin{array}{l}- \\
- \\
-\end{array}$ & $\begin{array}{r}6 \\
10 \\
13\end{array}$ & $\begin{array}{l}- \\
- \\
-\end{array}$ & $\begin{array}{l}- \\
- \\
-\end{array}$ & $\begin{array}{l}- \\
- \\
-\end{array}$ & $\begin{array}{l}- \\
- \\
-\end{array}$ & [128] \\
\hline PA-6 & IF-WS $2(40-180)$ & $\begin{array}{l}0.1 \\
0.5 \\
1 \\
2\end{array}$ & $\mathrm{mb}$ & $\begin{array}{l}36 \\
32 \\
30 \\
35\end{array}$ & $\begin{array}{l}4 \\
3 \\
6 \\
3\end{array}$ & $\begin{array}{r}0 \\
-1 \\
3 \\
-3\end{array}$ & $\begin{array}{r}1 \\
0 \\
5 \\
-2\end{array}$ & $\begin{array}{l}35 \\
37 \\
40 \\
40\end{array}$ & $\begin{array}{r}7 \\
9 \\
10 \\
9\end{array}$ & $\begin{array}{l}2 \\
6 \\
7 \\
5\end{array}$ & $\begin{array}{l}17 \\
12 \\
15 \\
17\end{array}$ & [167] \\
\hline PA-6 & $\mathrm{SiO}_{2}(20-100)$ & $\begin{array}{l}0.2 \\
0.8 \\
1.4 \\
2.7\end{array}$ & $\mathrm{st}+\mathrm{cm}$ & $\begin{array}{r}9 \\
18 \\
12 \\
4\end{array}$ & $\begin{array}{l}- \\
- \\
- \\
-\end{array}$ & $\begin{array}{r}4 \\
19 \\
5 \\
0\end{array}$ & $\begin{array}{l}- \\
- \\
- \\
-\end{array}$ & $\begin{array}{l}- \\
- \\
- \\
-\end{array}$ & $\begin{array}{l}- \\
- \\
- \\
-\end{array}$ & $\begin{array}{l}- \\
- \\
- \\
-\end{array}$ & $\begin{array}{l}- \\
- \\
- \\
-\end{array}$ & [171] \\
\hline PA-6 & $\mathrm{SiO}_{2}(80)$ & $\begin{array}{r}5 \\
10\end{array}$ & isp & $\begin{array}{l}10 \\
31\end{array}$ & $\begin{array}{l}- \\
-\end{array}$ & $\begin{array}{l}- \\
-\end{array}$ & $\begin{array}{l}11 \\
25\end{array}$ & $\begin{array}{l}- \\
-\end{array}$ & $\begin{array}{l}- \\
-\end{array}$ & $\begin{array}{l}- \\
-\end{array}$ & $\begin{array}{l}- \\
-\end{array}$ & [145] \\
\hline PA-6 & $\mathrm{SiO}_{2}(<50)$ & $\begin{array}{l}1 \\
2\end{array}$ & $\begin{array}{l}\mathrm{dm}+\mathrm{ext} \\
\text { isp }\end{array}$ & $\begin{array}{l}5 \\
8\end{array}$ & $\begin{array}{l}- \\
-\end{array}$ & $\begin{array}{l}- \\
-\end{array}$ & $\begin{array}{l}10 \\
11\end{array}$ & $\begin{array}{l}- \\
-\end{array}$ & $\begin{array}{l}- \\
-\end{array}$ & $\begin{array}{l}- \\
-\end{array}$ & $\begin{array}{l}- \\
-\end{array}$ & [172] \\
\hline
\end{tabular}


Table 1 (Continued)

\begin{tabular}{|c|c|c|c|c|c|c|c|c|c|c|c|c|}
\hline \multirow[t]{2}{*}{ Matrix } & \multirow{2}{*}{$\begin{array}{l}\text { Nanofiller (Particle } \\
\text { size/nm) }\end{array}$} & \multirow{2}{*}{$\begin{array}{l}\text { Nanofiller content } \\
\text { (wt.\%) }\end{array}$} & \multirow{2}{*}{$\begin{array}{l}\text { Processing } \\
\text { method }\end{array}$} & \multicolumn{4}{|l|}{ Nitrogen } & \multicolumn{5}{|c|}{ Oxygen or air atmosphere } \\
\hline & & & & $\Delta T_{1}\left({ }^{\circ} \mathrm{C}\right)$ & $\Delta T_{10}\left({ }^{\circ} \mathrm{C}\right)$ & $\Delta T_{50}\left({ }^{\circ} \mathrm{C}\right)$ & $\Delta T_{\mathrm{mr}}\left({ }^{\circ} \mathrm{C}\right)$ & $\Delta T_{\mathrm{i}}\left({ }^{\circ} \mathrm{C}\right)$ & $\Delta T_{10}\left({ }^{\circ} \mathrm{C}\right)$ & $\Delta T_{50}\left({ }^{\circ} \mathrm{C}\right)$ & $\Delta T_{\mathrm{mr}}\left({ }^{\circ} \mathrm{C}\right)$ & Ref. \\
\hline PA-6 & $\mathrm{SiO}_{2}(25-35)$ & $\begin{array}{l}1 \\
2 \\
3 \\
5\end{array}$ & isp & $\begin{array}{l}- \\
- \\
- \\
-\end{array}$ & $\begin{array}{l}15 \\
39 \\
79 \\
75\end{array}$ & $\begin{array}{r}-3 \\
-5 \\
4 \\
4\end{array}$ & $\begin{array}{l}- \\
- \\
- \\
-\end{array}$ & $\begin{array}{l}- \\
- \\
- \\
-\end{array}$ & $\begin{array}{l}- \\
- \\
- \\
-\end{array}$ & $\begin{array}{l}- \\
- \\
- \\
-\end{array}$ & $\begin{array}{l}- \\
- \\
- \\
-\end{array}$ & [149] \\
\hline PA-6 & $\mathrm{SiO}_{2}(10-20)$ & 0.2 & isp & 5 & - & - & 0 & - & - & - & - & [173] \\
\hline PA-6 & $\begin{array}{l}\text { APTES-modified } \\
\mathrm{SiO}_{2}(80)\end{array}$ & 5 & isp & 11 & - & - & 13 & - & - & - & - & [145] \\
\hline PA-6 & $\begin{array}{l}\text { AA-modified } \mathrm{SiO}_{2} \\
(10-20)\end{array}$ & 0.2 & isp & 8 & - & - & 0 & - & - & - & - & [173] \\
\hline PA-6 & $\begin{array}{l}\mathrm{CA} \text {-modified } \mathrm{SiO}_{2} \\
(10-20)\end{array}$ & 0.2 & isp & 11 & - & - & 2 & - & - & - & - & [173] \\
\hline PA-6 & $\mathrm{CaCO}_{3}(70)$ & 6 & $\mathrm{mb}$ & - & - & - & - & -5 & - & - & -25 & [175] \\
\hline PA- 6 & $\mathrm{CaCO}_{3}(11)$ & $\begin{array}{l}1 \\
2 \\
3 \\
4\end{array}$ & $m b+i n j$ & $\begin{array}{l}- \\
- \\
- \\
-\end{array}$ & $\begin{array}{l}- \\
- \\
- \\
-\end{array}$ & $\begin{array}{l}- \\
- \\
- \\
-\end{array}$ & $\begin{array}{r}89 \\
103 \\
109 \\
117\end{array}$ & $\begin{array}{l}- \\
- \\
- \\
-\end{array}$ & $\begin{array}{l}- \\
- \\
- \\
-\end{array}$ & $\begin{array}{l}- \\
- \\
- \\
-\end{array}$ & $\begin{array}{l}- \\
- \\
- \\
-\end{array}$ & {$[176]$} \\
\hline PA-6 & $\mathrm{CaCO}_{3}(23)$ & $\begin{array}{l}1 \\
2 \\
3 \\
4\end{array}$ & $m b+i n j$ & $\begin{array}{l}- \\
- \\
- \\
-\end{array}$ & $\begin{array}{l}- \\
- \\
-\end{array}$ & $\begin{array}{l}- \\
- \\
- \\
-\end{array}$ & $\begin{array}{l}47 \\
65 \\
79 \\
90\end{array}$ & $\begin{array}{l}- \\
- \\
-\end{array}$ & $\begin{array}{l}- \\
- \\
- \\
-\end{array}$ & $\begin{array}{l}- \\
- \\
- \\
-\end{array}$ & $\begin{array}{l}- \\
- \\
- \\
-\end{array}$ & {$[176]$} \\
\hline PA-6 & $\mathrm{TiO}_{2}$ & $\begin{array}{l}2 \\
4 \\
6 \\
8\end{array}$ & isp & $\begin{array}{l}- \\
- \\
- \\
-\end{array}$ & $\begin{array}{r}-3 \\
-2 \\
10 \\
-9\end{array}$ & $\begin{array}{r}23 \\
0 \\
12 \\
-6\end{array}$ & $\begin{array}{r}18 \\
1 \\
9 \\
-5\end{array}$ & $\begin{array}{l}- \\
- \\
- \\
-\end{array}$ & $\begin{array}{l}- \\
- \\
- \\
-\end{array}$ & $\begin{array}{l}- \\
- \\
- \\
-\end{array}$ & $\begin{array}{l}- \\
- \\
- \\
-\end{array}$ & [174] \\
\hline PA-6 & $\begin{array}{l}\text { APTES-modified } \\
\mathrm{TiO}_{2}\end{array}$ & $\begin{array}{l}2 \\
4 \\
6 \\
8\end{array}$ & isp & $\begin{array}{l}- \\
- \\
- \\
-\end{array}$ & $\begin{array}{r}3 \\
8 \\
9 \\
10\end{array}$ & $\begin{array}{r}-9 \\
7 \\
11 \\
24\end{array}$ & $\begin{array}{r}-1 \\
6 \\
9 \\
13\end{array}$ & $\begin{array}{l}- \\
- \\
- \\
-\end{array}$ & $\begin{array}{l}- \\
- \\
- \\
-\end{array}$ & $\begin{array}{l}- \\
- \\
- \\
-\end{array}$ & $\begin{array}{l}- \\
- \\
- \\
-\end{array}$ & [174] \\
\hline PPS & IF-WS $(40-180)$ & $\begin{array}{l}0.1 \\
0.5 \\
1 \\
2 \\
4 \\
8\end{array}$ & $\mathrm{mb}$ & $\begin{array}{r}2 \\
-1 \\
2 \\
-1 \\
7 \\
4\end{array}$ & $\begin{array}{l}1 \\
0 \\
0 \\
0 \\
2 \\
2\end{array}$ & $\begin{array}{r}88 \\
95 \\
30 \\
163 \\
141 \\
-\end{array}$ & $\begin{array}{r}-1 \\
0 \\
-12 \\
10 \\
-10 \\
7\end{array}$ & $\begin{array}{l}3 \\
2 \\
3 \\
3 \\
2 \\
2\end{array}$ & $\begin{array}{l}0 \\
2 \\
3 \\
3 \\
2 \\
3\end{array}$ & $\begin{array}{r}1 \\
4 \\
5 \\
5 \\
33 \\
44\end{array}$ & $\begin{array}{r}3 \\
1 \\
3 \\
3 \\
33 \\
50\end{array}$ & {$[177]$} \\
\hline PPS & $\mathrm{Fe}_{3} \mathrm{O}_{4}$ & $\begin{array}{l}20 \\
30 \\
40\end{array}$ & $m b+c m$ & $\begin{array}{r}9 \\
16 \\
18\end{array}$ & $\begin{array}{l}10 \\
14 \\
17\end{array}$ & $\begin{array}{l}- \\
- \\
-\end{array}$ & $\begin{array}{r}5 \\
9 \\
12\end{array}$ & $\begin{array}{l}- \\
- \\
-\end{array}$ & $\begin{array}{l}- \\
- \\
-\end{array}$ & $\begin{array}{l}- \\
- \\
-\end{array}$ & $\begin{array}{l}- \\
- \\
-\end{array}$ & [178] \\
\hline PEEK & IF-WS $(40-180)$ & $\begin{array}{l}0.1 \\
0.5 \\
1 \\
2\end{array}$ & $\mathrm{bml}+\mathrm{mb}$ & $\begin{array}{l}19 \\
32 \\
39 \\
49\end{array}$ & $\begin{array}{l}18 \\
21 \\
45 \\
53\end{array}$ & $\begin{array}{l}- \\
- \\
-\end{array}$ & $\begin{array}{l}19 \\
34 \\
45 \\
59\end{array}$ & $\begin{array}{l}21 \\
41 \\
59 \\
55\end{array}$ & $\begin{array}{l}12 \\
31 \\
47 \\
59\end{array}$ & $\begin{array}{l}20 \\
44 \\
58 \\
62\end{array}$ & $\begin{array}{l}12 \\
30 \\
48 \\
57\end{array}$ & [57] \\
\hline
\end{tabular}


Table 1 (Continued)

\begin{tabular}{|c|c|c|c|c|c|c|c|c|c|c|c|c|}
\hline \multirow[t]{2}{*}{ Matrix } & \multirow{2}{*}{$\begin{array}{l}\text { Nanofiller (Particle } \\
\text { size/nm) }\end{array}$} & \multirow{2}{*}{$\begin{array}{l}\text { Nanofiller content } \\
\text { (wt.\%) }\end{array}$} & \multirow{2}{*}{$\begin{array}{l}\text { Processing } \\
\text { method }\end{array}$} & \multicolumn{4}{|l|}{ Nitrogen } & \multicolumn{5}{|c|}{ Oxygen or air atmosphere } \\
\hline & & & & $\Delta T_{\mathrm{i}}\left({ }^{\circ} \mathrm{C}\right)$ & $\Delta T_{10}\left({ }^{\circ} \mathrm{C}\right)$ & $\Delta T_{50}\left({ }^{\circ} \mathrm{C}\right)$ & $\Delta T_{\mathrm{mr}}\left({ }^{\circ} \mathrm{C}\right)$ & $\Delta T_{\mathrm{i}}\left({ }^{\circ} \mathrm{C}\right)$ & $\Delta T_{10}\left({ }^{\circ} \mathrm{C}\right)$ & $\Delta T_{50}\left({ }^{\circ} \mathrm{C}\right)$ & $\Delta T_{\mathrm{mr}}\left({ }^{\circ} \mathrm{C}\right)$ & Ref. \\
\hline \multirow[t]{3}{*}{ PEEK ${ }^{\mathrm{a}}$} & IF-WS $2(80-220)$ & 1 & aed & - & - & - & - & 5 & - & - & 15 & {$[58]$} \\
\hline & & 5 & & - & - & - & - & 30 & - & - & 20 & \\
\hline & & 20 & & - & - & - & - & 65 & - & - & 25 & \\
\hline \multirow[t]{4}{*}{ PEEK } & $\mathrm{SiO}_{2}(30)$ & 2.5 & $\mathrm{ult}+\mathrm{cm}$ & - & 11 & - & 15 & - & - & - & - & [159] \\
\hline & & 5 & & - & 14 & - & 20 & - & - & - & - & \\
\hline & & 7.5 & & - & 19 & - & 23 & - & - & - & - & \\
\hline & & 10 & & - & 19 & - & 25 & - & - & - & - & \\
\hline \multirow[t]{4}{*}{ PEEK } & $\mathrm{SiO}_{2}(30)$ & 2.5 & $\mathrm{ult}+\mathrm{cm}$ & - & 25 & - & 25 & - & - & - & - & [152] \\
\hline & & 5 & & - & 27 & - & 27 & - & - & - & - & \\
\hline & & 7.5 & & - & 29 & - & 30 & - & - & - & - & \\
\hline & & 10 & & - & 38 & - & 35 & - & - & - & - & \\
\hline \multirow[t]{4}{*}{ PEEK } & $\begin{array}{l}\text { SA-modified } \mathrm{SiO}_{2} \\
(30)\end{array}$ & 2.5 & $\mathrm{ult}+\mathrm{cm}$ & - & 0 & - & 0 & - & - & - & - & [159] \\
\hline & & 5 & & - & 3 & - & 10 & - & - & - & - & \\
\hline & & 7.5 & & - & 17 & - & 18 & - & - & - & - & \\
\hline & & 10 & & - & 20 & - & 24 & - & - & - & - & \\
\hline \multirow[t]{4}{*}{ PEEK } & $\mathrm{Al}_{2} \mathrm{O}_{3}(30)$ & 2.5 & $\mathrm{ult}+\mathrm{cm}$ & - & 27 & - & 23 & - & - & - & - & [152] \\
\hline & & 5. & & - & 20 & - & 20 & - & - & - & - & \\
\hline & & 7.5 & & - & 26 & - & 25 & - & - & - & - & \\
\hline & & 10 & & - & 34 & - & 35 & - & - & - & - & \\
\hline \multirow[t]{7}{*}{ PEEK } & $\mathrm{Al}_{2} \mathrm{O}_{3}(39)$ & 1.25 & $\mathrm{ult}+\mathrm{cm}$ & - & 8 & - & 8 & - & 24 & - & 26 & [150] \\
\hline & & 2.5 & & - & 2 & - & 6 & - & 8 & - & -2 & \\
\hline & & 5 & & - & 0 & - & 8 & - & 11 & - & 14 & \\
\hline & & 7.5 & & - & 3 & - & 6 & - & 22 & - & 34 & \\
\hline & & 10 & & - & 14 & & 10 & & 28 & & 51 & \\
\hline & & 20 & & - & 5 & - & 6 & - & 11 & - & 51 & \\
\hline & & 30 & & - & 10 & - & 6 & - & 24 & - & 44 & \\
\hline \multirow[t]{4}{*}{ PEEK } & $\mathrm{Si}_{3} \mathrm{~N}_{4}(20-30)$ & 5 & $\mathrm{ult}+\mathrm{cm}$ & - & -2 & - & -5 & - & - & - & - & [180] \\
\hline & & 10 & & - & 5 & - & -4 & - & - & - & - & \\
\hline & & 20 & & - & 0 & - & -5 & - & - & - & - & \\
\hline & & 30 & & - & 2 & - & -6 & - & - & - & - & \\
\hline \multirow[t]{5}{*}{ PEEK } & AlN & 5.2 & $\mathrm{st}+\mathrm{cm}$ & - & - & - & - & - & 5 & 15 & 8 & [179] \\
\hline & & 9 & & - & - & - & - & - & 5 & 25 & 25 & \\
\hline & & 21 & & - & - & - & - & - & 10 & 15 & 20 & \\
\hline & & 31 & & - & - & - & - & - & 10 & 25 & 48 & \\
\hline & & 48 & & - & - & - & - & - & 10 & 30 & 30 & \\
\hline
\end{tabular}

Key: $\mathrm{mb}=$ melt blending; $\mathrm{cm}=$ compression molding; inj = injection molding; $\mathrm{st}=$ stirring; isp = in situ polymerization; $\mathrm{dm}=\mathrm{dry}$ mixing; ext = extrusion; bml=ball milling; aed = aerosol assisted deposition;

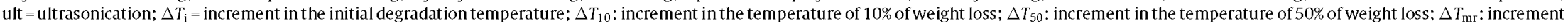
in the temperature of maximum rate of weight loss.

a Nanocomposite coating. 
comparable to those attained when conventional NAs for iPP are employed [181-184], and clearly higher than the values obtained in other iPP nanocomposites [185,186]. The presence of IF-WS 2 nanoparticles reduces the tendency for recrystallization during the heating process subsequent to crystallization from the melt [187], once more reflecting its nucleating activity and the larger size and stability of the iPP crystals developed at a crystallization temperature higher than that of neat iPP.

Under isothermal crystallization conditions the crystallization temperature range for iPP/IF-WS 2 nanocomposites is higher with notably shorter crystallization times. Fig. 20b shows the time required to reach $10 \%$ of crystalline transformation, $\tau_{0.1}$ represented against the crystallization temperature and composition, demonstrating the increase in the crystallization rate due to the presence of the nanofiller [188]. On addition of IF-WS 2 the value of the fold surface free energy of the crystalline lamellae [189], $\sigma_{\mathrm{e}}$ varies from $91.9 \mathrm{erg} \mathrm{cm}^{-2}$ for neat iPP to $91.6 \mathrm{erg} \mathrm{cm}^{-2}$ with $0.1 \mathrm{wt} . \% \mathrm{IF}_{-} \mathrm{WS}_{2}$ content, $71.0 \mathrm{erg} \mathrm{cm}^{-2}$ with $1 \mathrm{wt} . \%$ IF$\mathrm{WS}_{2}$ and $70.1 \mathrm{erg} \mathrm{cm}^{-2}$ with $2 \mathrm{wt} . \%$. As the IF-WS 2 content is increased there is a clear decrease in $\sigma_{\mathrm{e}}$ that involves a reduction of the nucleation free energy and a concommitant increase in the isothermal crystallization rate. Thus, IF-WS ${ }_{2}$ acts as a highly efficient NA for the monoclinic $\alpha$-form of iPP, affecting all the crystallization parameters without modification of the crystal structure of iPP.

Nucleating effects on the crystallization of iPP have also been described with other spherical or pseudo-spherical inorganic nanoparticles. Jain et al. [185] analyzed the crystallization behavior of iPP in iPP/silica nanocomposties prepared in situ via a solid-state modification and sol-gel reaction and observed that $\mathrm{SiO}_{2}$ nanoparticles with an average size of $30-80 \mathrm{~nm}$ nucleate iPP crystallization reducing the activation energy for the process with a NE of around $20 \%$ for a concentration of 5 wt.\% nanofiller. However, at these nanofiller levels the formation of the $\beta$ or trigonal polymorph of iPP is induced. On the other hand, Dougnac et al. [190] reported the absence of nucleating activity in iPP of $\mathrm{SiO}_{2}$ nanospheres of between 12 and $150 \mathrm{~nm}$ diameter obtained by a sol-gel method. Asuka et al. [95] studied the effect of particle size, between 16 and $100 \mathrm{~nm}$, and composition on the crystallization of iPP in totally hydroxylated $\mathrm{SiO}_{2}$, and found a significant reduction in the spherullitic growth rate of iPP with increasing silica concentration and decreasing particle size. The same authors surface modified silica nanoparticles with trimethylchlorosilane and n-octyldimethylchlorosilane coupling agents with a view to improve the miscibility with the hydrophobic iPP matrix [99] and found that the hydrophobicity of $\mathrm{SiO}_{2}$ provoked an increase in the crystalline nucleation of iPP. However, as was the case for untreated nanoparticles the spherullitic growth rate diminished significantly with increasing silica concentration and decreasing particle size, and was practically independent of the surface modification due to confinement in the interparticle spaces. In other studies, the use of silane coupling agents such as dichlorodimethyl silane (DCMS) has led to higher iPP crystallization rates in PNCs with nanoparticles of $12 \mathrm{~nm}$ average size, reaching maximum nucleation efficiencies for a concentration of $7 \mathrm{wt} . \%$ nanofiller [98]. In other cases previous surface

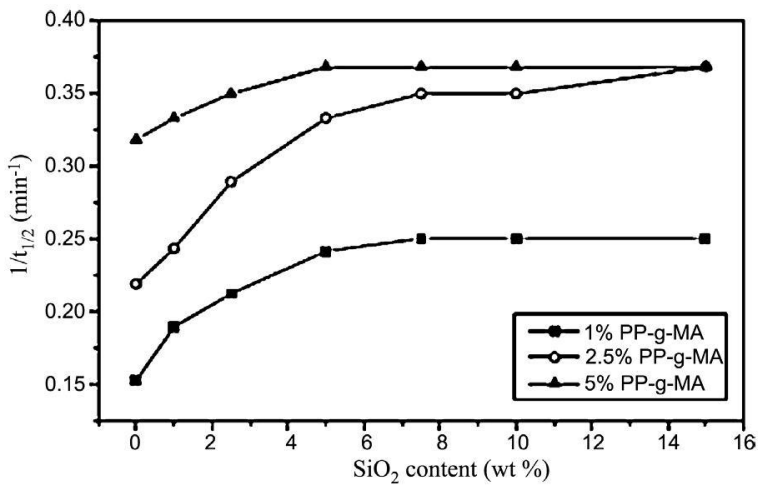

Fig. 21. Crystallization rate expressed as the inverse of crystallization half time versus the content of $\mathrm{SiO}_{2}$ nanoparticles.

Reprinted with permission [192]. Copyright 2006, Elsevier.

activation of the $\mathrm{SiO}_{2}$ nanoparticles and the subsequent use of coupling agents with different functional groups such as APTES, GPTMS, trimethoxysilylpropyl methacrylate (TMPM) or DCMS did not produce significant variations in the melting and crystallization parameters of iPP, nor did they affect the crystalline microstructure of the polymeric matrix [170]. On the contrary, the combined activity of a trialcoxysilane coupling agent with an anionic dispersing agent such as sodium dodecylbenzene sulfonate (SDBS) has been reported to provoke significant increases both in the crystallization temperature and the crystallinity of iPP, whilst maintaining its crystalline microstructure [191].

The application of strategies based on the use of compatibilizing agents like PP-g-MAH has demonstrated that both the concentration and the degree of grafting affects the nucleating capacity of $\mathrm{SiO}_{2}$ nanoparticles $[105,192,193]$, as can be observed for example in Fig. 21 that shows the variation of the crystallization peak temperature $\left(T_{\mathrm{C}}\right)$ of iPP as a function of the concentration of nano- $\mathrm{SiO}_{2}$ for different concentrations of PP-g-MAH with a degree of grafting of $0.6 \%$ [192].

It has also been described in some cases that the nucleating activity of $\mathrm{SiO}_{2}$ nanoparticles can be reduced by prior surface modification via graft polymerization with monomers such as styrene, methyl methacrylate, ethyl acrylate o butyl acrylate [109], and was favored when the surface grafting was undertaken with PGMA via radical polymerization in solution and a subsequent interfacial reaction with $\mathrm{PP}-\mathrm{g}-\mathrm{NH}_{2}$ in the molten phase, which acts as a compatibilization agent with the iPP matrix [115]. Ionic liquids such as 1-decyl-3-methylimidazolium tetrafluorborate have also been used as coupling agents in the preparation of $\mathrm{iPP} / \mathrm{SiO}_{2}$ nanocomposites and an important nucleating activity observed, represented by an $8^{\circ} \mathrm{C}$ increase in $T_{c}$ with respect to that of neat iPP [101]. In Table 2 some of the crystallization parameters for the aforementioned $\mathrm{iPP} / \mathrm{SiO}_{2}$ nanocomposites are summarized, where $\Delta T_{c}, \Delta \chi_{c}$ and $\Delta T_{m}$, represent the variation in the crystallization peak temperature, degree of crystallinity and melting temperature, respectively for iPP in the nanocomposites with respect to those in neat iPP.

Different authors have studied the crystallization behavior of iPP in the presence of $\mathrm{CaCO}_{3}$ nanoparticles. 
Table 2

Change in the crystallization peak temperature $\left(\Delta T_{\mathrm{c}}\right)$, crystallinity $\left(\Delta \chi_{\mathrm{c}}\right)$ and melting temperature $\left(\Delta T_{\mathrm{c}}\right)$ for different iPP/SiO $\mathrm{S}_{2}$ nanocomposites.

\begin{tabular}{|c|c|c|c|c|c|c|c|}
\hline Nanofiller type & Nanofiller size $(\mathrm{nm})$ & Nanofiller content (\%) & Characteristics & $\Delta T_{\mathrm{c}}\left({ }^{\circ} \mathrm{C}\right)$ & $\Delta X_{c}(\%)$ & $\Delta T_{\mathrm{m}}\left({ }^{\circ} \mathrm{C}\right)$ & Ref. \\
\hline $\mathrm{SiO}_{2}$ & $30-80$ & $\begin{array}{l}0.2 \\
0.5 \\
1 \\
1.5\end{array}$ & - & $\begin{array}{l}2.8 \\
6.2 \\
4.8 \\
3.2\end{array}$ & $\begin{array}{l}1.3 \\
3.4 \\
5.6 \\
1.3\end{array}$ & $\begin{array}{l}1.1 \\
1.6 \\
1.5 \\
1.3\end{array}$ & [185] \\
\hline $\mathrm{SiO}_{2}$ & - & $\begin{array}{l}2 \\
4 \\
5\end{array}$ & $\begin{array}{l}\text { KH-550 } \\
\text { SDBS }\end{array}$ & $\begin{array}{l}5 \\
6.8 \\
7.2\end{array}$ & $\begin{array}{l}8 \\
6 \\
6.9\end{array}$ & $\begin{array}{r}0.9 \\
-0.3 \\
-0.6\end{array}$ & [191] \\
\hline $\mathrm{SiO}_{2}$ & 12 & 3 & KH570 & 1.1 & 0.5 & -0.6 & {$[115]$} \\
\hline \multirow[t]{4}{*}{$\mathrm{SiO}_{2}-\mathrm{g}$-PGMA } & 12 & $\begin{array}{l}3 \\
3 / 10\end{array}$ & $\begin{array}{l}\text { KH570 } \\
\text { KH570 } \\
\text { PP-g-MAH }\end{array}$ & $\begin{array}{l}2.8 \\
9.8\end{array}$ & $\begin{array}{r}-2.1 \\
6.8\end{array}$ & $\begin{array}{l}0.5 \\
0.9\end{array}$ & [115] \\
\hline & & $3 / 10$ & $\begin{array}{l}\mathrm{KH} 570 \\
\mathrm{PP}-\mathrm{g}-\mathrm{NH}_{2}\end{array}$ & 9.7 & 3.8 & 1 & \\
\hline & & $3 / 10 / 0.17$ & $\begin{array}{l}\text { KH570 } \\
\text { PP-g-MAH } \\
\text { HDMA }\end{array}$ & 8 & 2.3 & 1.2 & \\
\hline & & $\begin{array}{l}1 / 10 \\
2 / 10 \\
3 / 10 \\
4 / 10\end{array}$ & $\begin{array}{l}\mathrm{KH} 570 \\
\mathrm{PP}-\mathrm{g}-\mathrm{NH}_{2}\end{array}$ & $\begin{array}{r}9.6 \\
9.9 \\
9.7 \\
11\end{array}$ & $\begin{array}{l}3.6 \\
5.4 \\
3.8 \\
7.3\end{array}$ & $\begin{array}{l}1 \\
0.8 \\
1 \\
0.6\end{array}$ & \\
\hline $\mathrm{SiO}_{2}$ & $\begin{array}{r}12 \\
30 \\
36 \\
44 \\
70 \\
150\end{array}$ & $\begin{array}{l}1 \\
1 \\
1 \\
1 \\
1 \\
1\end{array}$ & $\mathrm{PP}(\mathrm{M}=150,000)$ & $\begin{array}{l}-1 \\
-3 \\
-3 \\
-3 \\
-2 \\
-2\end{array}$ & $\begin{array}{l}0 \\
4 \\
0 \\
2 \\
1 \\
3\end{array}$ & $\begin{array}{l}-3 \\
-1 \\
-3 \\
-2 \\
-1 \\
-1\end{array}$ & {$[190]$} \\
\hline $\mathrm{SiO}_{2}$ & $\begin{array}{r}12 \\
30 \\
36 \\
44 \\
70 \\
150\end{array}$ & $\begin{array}{l}1 \\
1 \\
1 \\
1 \\
1 \\
1\end{array}$ & $\mathrm{PP}(\mathrm{M}=250,000)$ & $\begin{array}{r}-1 \\
0 \\
0 \\
0 \\
0 \\
0\end{array}$ & $\begin{array}{r}3 \\
-3 \\
1 \\
3 \\
4 \\
3\end{array}$ & $\begin{array}{r}-4 \\
0 \\
-2 \\
-1 \\
-2 \\
-2\end{array}$ & {$[190]$} \\
\hline $\mathrm{SiO}_{2}$ (Activated) & 7 & $\begin{array}{l}2 \\
2 \\
2 \\
2\end{array}$ & $\begin{array}{l}\text { APTES } \\
\text { GPTMS } \\
\text { TMPM } \\
\text { DCMS }\end{array}$ & $\begin{array}{l}2 \\
0 \\
1 \\
1\end{array}$ & $\begin{array}{r}-0.3 \\
-1.4 \\
-0.5 \\
0.5\end{array}$ & $\begin{array}{r}0 \\
0 \\
0 \\
-1\end{array}$ & {$[170]$} \\
\hline $\mathrm{SiO}_{2}$ (Unactivated) & - & $\begin{array}{l}2 \\
2 \\
2 \\
2\end{array}$ & $\begin{array}{l}\text { APTES } \\
\text { GPTMS } \\
\text { TMPM } \\
\text { DCMS }\end{array}$ & $\begin{array}{l}1 \\
0 \\
0 \\
1\end{array}$ & $\begin{array}{l}-0.3 \\
-1.4 \\
-1.7 \\
-0.7\end{array}$ & $\begin{array}{l}1 \\
1 \\
1 \\
1\end{array}$ & {$[170]$} \\
\hline
\end{tabular}

KH-550: Trial koxysilane coupling agent; SDBD: Sodium dodecylbenzene sulfonate; KH-570: $\gamma$-metacryloxypropyl trimethoxysilane; PGMA: Poly(glycidyl methacrylate); PP-g-MAH: Maleic anhydride grafted PP; PP-g-NH $\mathrm{N}_{2}$ : Aminated PP; HMDA: Hexamethylendiamine; APTES: Aminopropyltriethoxysilane; GPTMS: Glycidyloxypropyltrimethoxysilane; TMPM: Trimethoxysilylp ropylmethacrylate; DCMS: Dichlorodimethylsilane.

Chan et al. [194] described an increase of $10^{\circ} \mathrm{C}$ in $T_{\mathrm{C}}$ with little variation in $\chi_{c}$ using nanoparticles of around $44 \mathrm{~nm}$ in size, and the development of small fractions of the $\beta$ polymorph of iPP for concentrations of between 4.8 and 13.2\% nanofiller. Mishra et al. [195] also observed the development of the trigonal polymorph for nanoparticle sizes between 21 and $39 \mathrm{~nm}$ as well as a considerable increase in $T_{\mathrm{m}}$ with the reduction in the size of the nanoparticles for compositions between 2 and $10 \%$. Eiras et al. [196] have also reported the formation of the $\beta$ polymorph and an increase in both $T_{\mathrm{c}}$ and $\chi_{\mathrm{c}}$ with increasing content of $70-90 \mathrm{~nm}$ nanoparticles.

The surface modification of $\mathrm{CaCO}_{3}$ nanoparticles with fatty acids such as $\mathrm{SA}$ or oleic $(\mathrm{OA})$ leads to dispersed results. In some cases the formation of the $\beta$ polymorph and an important increase in $T_{\mathrm{C}}$ with respect to nanocomposites with unmodified nanoparticles has been reported to be a consequence of the improved dispersion of the nanofiller in the iPP matrix [197-199]. On the contrary, Shentu et al. [197] and Avella et al. [200] indicated that the addition of fatty acid coated $\mathrm{CaCO}_{3}$ reduced efficient nucleation due to the reduction in SSA, and Li et al. [201] have observed a nucleating effect but with the formation of the monoclinic polymorph in nanocomposites with a composition of up to $50 \%$. Other authors, using different strategies such as surface modification with aluminate type agents, with or without SA [116], non ionic dispersing agents such as polyoxyethylene nonyphenol [202] or silanes [122] have also described nucleating activity and the formation of the $\beta$ polymorph in iPP.

The modification of the surface activity of the $\mathrm{CaCO}_{3}$ nanoparticles via reaction with compatibilizing agents in 
Table 3

Change in the crystallization peak temperature $\left(\Delta T_{\llcorner}\right)$, crystallinity $\left(\Delta \chi_{c}\right)$ and melting temperature $\left(\Delta T_{\llcorner}\right)$for different iPP/CaCO${ }_{3}$ nanocomposites.

\begin{tabular}{|c|c|c|c|c|c|c|c|}
\hline Nanofiller type & Nanofiller size $(\mathrm{nm})$ & Nanofiller content $\%$ ) & Characteristics & $\Delta T_{\mathrm{c}}\left({ }^{\circ} \mathrm{C}\right)$ & $\Delta \chi_{c}(\%)$ & $\Delta T_{\mathrm{m}}\left({ }^{\circ} \mathrm{C}\right)$ & Ref. \\
\hline \multirow[t]{3}{*}{$\mathrm{CaO}_{3}$} & 44 & 4.8 & - & 10.2 & -10.2 & 0 & [194] \\
\hline & & 9.2 & & 9.5 & -9.5 & 0 & \\
\hline & & 13.1 & & 10.7 & -10.7 & 0 & \\
\hline \multirow[t]{3}{*}{$\mathrm{CaO}_{3}$} & 39 & 6 & - & - & -1.4 & 13.9 & [195] \\
\hline & 30 & & & - & -2.9 & 16.4 & \\
\hline & 21 & & & - & -2.9 & 17.5 & \\
\hline \multirow[t]{4}{*}{$\mathrm{CaO}_{3}$} & $70-90$ & 3 & - & 9 & 2 & 0 & [196] \\
\hline & & 5 & & 7 & 7 & -1 & \\
\hline & & 7 & & 7 & 8 & 0 & \\
\hline & & 10 & & 7 & 6 & 0 & \\
\hline \multirow[t]{2}{*}{$\mathrm{CaO}_{3}$} & $60-80$ & 3 & Aluminate coupling agent & 5.9 & 1.8 & -0.4 & [116] \\
\hline & & & Aluminate coupling agent SA & 3.4 & 1.3 & 0 & \\
\hline \multirow[t]{12}{*}{$\mathrm{CaO}_{3}$} & $40-60$ & 2 & - & -1.4 & - & 0 & [186] \\
\hline & & 5 & & -0.7 & - & 0 & \\
\hline & & 10 & & 3.3 & - & 1.2 & \\
\hline & & 40 & & 5.6 & - & 1.3 & \\
\hline & & $40 / 5$ & PP-g-AA & 2.8 & - & 0.3 & \\
\hline & & $40 / 10$ & & 2.6 & - & 0.2 & \\
\hline & & $40 / 2$ & AA & -4.6 & - & -0.5 & \\
\hline & & $40 / 4$ & & -1.6 & - & -0.4 & \\
\hline & & $40 / 6$ & & 3.8 & - & 1.3 & \\
\hline & & $40 / 2 / 0.1$ & AA/DCP & 3.6 & - & 0 & \\
\hline & & $40 / 4 / 0.1$ & & 1.4 & - & 0.2 & \\
\hline & & $40 / 6 / 0.1$ & & 2.2 & - & -0.3 & \\
\hline \multirow[t]{2}{*}{$\mathrm{CaO}_{3}$} & 80 & 1 & - & 2.1 & 2.4 & - & [197] \\
\hline & & & $\mathrm{OA}$ & 8.9 & 9.3 & - & \\
\hline \multirow[t]{2}{*}{$\mathrm{CaO}_{3}$} & 44 & 2 & SA & 10 & 0 & - & [198] \\
\hline & & & PP-g-MAH & & & & \\
\hline \multirow[t]{7}{*}{$\mathrm{CaO}_{3}$} & - & 10 & SA (2\%) & 6.3 & - & - & [199] \\
\hline & & 20 & & 5.8 & - & - & \\
\hline & & 25 & & 5.7 & - & - & \\
\hline & & 30 & & 6.8 & - & - & \\
\hline & & 10 & SA (6\%) & 7.8 & - & - & \\
\hline & & 20 & & 6.8 & - & - & \\
\hline & & 30 & & 7.8 & - & - & \\
\hline \multirow[t]{5}{*}{$\mathrm{CaO}_{3}$} & - & $5 / 1$ & MAH & 0.3 & - & 2.7 & [203] \\
\hline & & $5 / 1.5$ & & -0.9 & - & -0.2 & \\
\hline & & $5 / 2$ & & 4.1 & - & 0.5 & \\
\hline & & $5 / 2.5$ & & 3.7 & - & 0.1 & \\
\hline & & $5 / 3$ & & 4.6 & - & 1.5 & \\
\hline
\end{tabular}

PP-g-AA: Acrylic acid grafted PP; PP-g-MAH: Maleic anhydride grafted PP; AA: Acrylic Acid; DCP: Dicumyl peroxide; MAH: Maleic Anhydride; OA: Oleic Acid; SA: Stearic Acid.

order to improve interfacial adhesion between nanofiller and polyolefin matrix has also been amply exploited.Avella et al. $[169,200]$ described improvements in the nucleating activity of $\mathrm{CaCO}_{3}$ nanoparticles coated with a graft copolymer PP-g-MAH due to the inherent crystallizability of the copolymer. In the same vein, Huang et al. [203] showed an increase in $\chi_{\mathrm{c}}, T_{\mathrm{C}}$ and crystallization rate constant with increasing concentration of maleic anhydride (MAH). Wang et al. [204] described the influence of three types of MAH compatibilizer, PP-g-MAH, polyolefin elastomer (POE-g$\mathrm{MAH}$ ) and ethylene-co-vinyl acetate (EVA-g-MAH), and in all cases the crystallization rate increased and the interfacial basal free energy of the crystallites diminished to favor the formation of the trigonal polymorph. This effect is dependant on the interfacial interaction between iPP and the compatibilizing agent such that an increase in the miscibility favors heterogeneous nucleation. Lin et al. [186] also found a nucleating effect using polypropylene grafted with acrylic acid, PP-g-AA that saturated with increasing compatibilizing agent concentration. When the copolymer is generated in situ via reaction of the AA monomer in the presence of dicumyl peroxide (DCP) the nucleating effect observed was superior to that produced in the absence of peroxide. Table 3 summarizes some of the crystallization parameters for the aforementioned iPP/ $\mathrm{CaCO}_{3}$ nanocomposites.

Spherical $\mathrm{Al}_{2} \mathrm{O}_{3}$ of average size around $47 \mathrm{~nm}$ pretreated with a silane coupling agent behave as NAs for the crystallization of iPP in the composition range $1.5-5 \%$ [134], provoking an increase in the $T_{\mathrm{C}}$ with a maintained level of crystallinity, leading to an important reduction in spherulite size as a consequence of the increase in nucleation density with respect to that of neat iPP, Fig. 22a and $\mathrm{b}$. This nucleating activity is accompanied by the development of trigonal polymorphism as is observed in Fig. 22c by the appearance of a reflection at $2 \theta \sim 16^{\circ}$ corresponding 

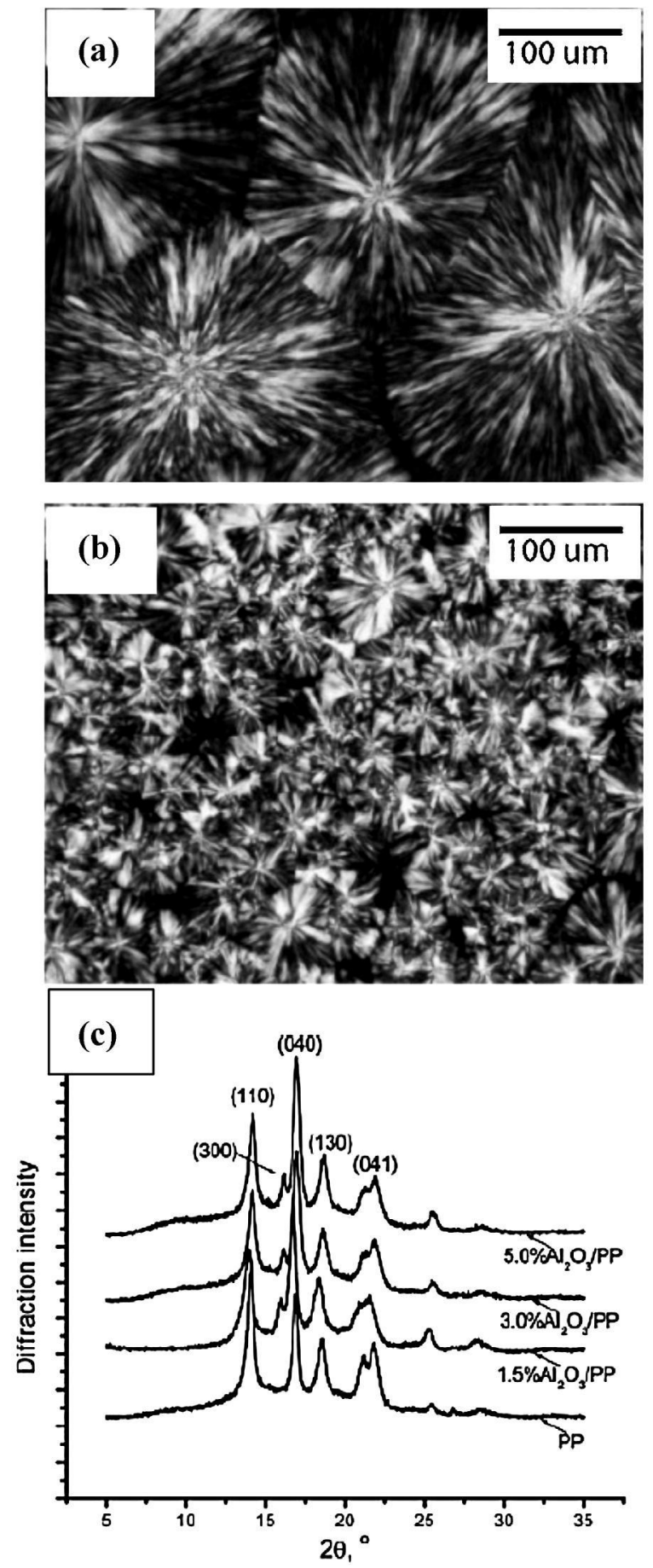

Fig. 22. POM images of (a) neat PP and (b) 3.0 wt. $\% \mathrm{Al}_{2} \mathrm{O}_{3}$ nanocomposite, and (c) WAXD curves for neat iPP and iPP/ $\mathrm{Al}_{2} \mathrm{O}_{3}$ nanocomposites of varied composition.

Reprinted with permission [134]. Copyright 2005, John Wiley and Sons.

to the (300) plane of the $\beta$ crystalline modification in the corresponding X-ray diffractograms.

There is abundant information in the literature on the crystallization behavior of iPP in its nanocomposites with $\mathrm{ZnO}$, although some reports are contradictory. In this respect, D'Haese et al. [205] have described a NE between 4 and $9 \%$ on the Lotz scale in nanocomposites with $1 \% \mathrm{ZnO}$ of various sizes between 35 and $500 \mathrm{~nm}$, and other authors have encountered slight nucleation effects without modification of the monoclinic structure of iPP $[127,129]$. Increases in $T_{\mathrm{C}}$ of around $4-5^{\circ} \mathrm{C}$ and $8^{\circ} \mathrm{C}$ have also been described for $\mathrm{PP} /$ methylated $\mathrm{ZnO}$ and $\mathrm{PP}-\mathrm{g}$ $\mathrm{MAH} /$ methylated-ZnO nanocomposites, respectively, with the crystallinity developed around $10 \%$ higher due to the increase of the interaction level generated by the presence of methyl groups on the nanoparticle surface [206]. Zhao et al. [128] have also described a nucleating effect in iPP with $\mathrm{ZnO}$ nanoparticles that were pre-treated with an organo-silane coupling agent, but with the formation of the $\beta$ polymorph in iPP. On the contrary, very recently Lepot et al. [130] observed a reduction in both $\chi_{\mathrm{c}}$ and $T_{\mathrm{m}}$ in biaxially oriented films prepared from iPP nanocomposites with $\mathrm{ZnO}$ nanoparticles of $50-70 \mathrm{~nm}$ average size at compositions between 2 and $7.5 \%$ nanofiller, explained to be due to the restriced molecular mobility of the iPP chains because of the improved interaction with the $\mathrm{ZnO}$ nanoparticles.

Suphaphol et al. [207] described the crystallization behavior of iPP in the presence of $\mathrm{TiO}_{2}$ nanoparticles of around $50 \mathrm{~nm}$ size, between 5 and 30\% nanofiller concentration and analyzed the influence of surface coating the nanoparticles with $\mathrm{SiO}_{2}$ or $\mathrm{SA}$. In the $\mathrm{iPP} / \mathrm{TiO}_{2}$ nanocomposites a nucleating effect was manifested by a concentration independent increase in $T_{C}$, whereas for $i \mathrm{PP} / \mathrm{TiO}_{2}-\mathrm{SA}$ nanocomposites no nucleation effect was observed. In both cases the monoclinic structure was maintained. On the other hand, in the $\mathrm{iPP} / \mathrm{TiO}_{2}-\mathrm{SiO}_{2}$ nanocomposites the nucleating effect is important, with around $8^{\circ} \mathrm{C}$ increase in $T_{\mathrm{C}}$ for a $30 \%$ of silica coated nanofiller, obtaining both monoclinic and trigonal polymorphs in the matrix. This phenomenon can be justified by the existence of two nucleation centres on the $\mathrm{TiO}_{2}$ nanoparticles associated with an uneven silica coating.

Spherical nanoparticles of $\mathrm{Si}_{3} \mathrm{~N}_{4}$ also exert a nucleating effect on the crystallization of iPP. Nanocomposites with $2 \%$ composition of $\mathrm{Si}_{3} \mathrm{~N}_{4}$ particles of between 15 and $30 \mathrm{~nm}$ increase $T_{\mathrm{C}}$ by $5^{\circ} \mathrm{C}$ and $\chi_{c}$ by around $4 \%$ [208]. Other authors have also shown a nucleating effect using silane coupling agents, although the efficiency did not increase with nanofiller content [209], and in some cases the disappearance of trigonal polymorphism in neat iPp was observed with increased amounts of $\mathrm{Si}_{3} \mathrm{~N}_{4}$ in the nanocomposite [124].

Spherical nanoparticles of carbon nitride also act as NAs in the crystallization of iPP [126] and both $T_{C}$ and $\chi_{c}$ increase with composition, up to $2 \% C_{3} \mathrm{~N}_{4}$, whilst maintaining the monoclinic structure. The variations in the crystallization activation energy as a function of composition illustrate the balance and competition the nanoparticles exert on the crystalline nucleation and macromolecular chain transport in the growing crystal.

In the case of physical blends of iPP with fullerene- $\mathrm{C}_{60}$ prepared both in solution and the melt no variations in the crystallization and melting parameters of iPP have been observed [142]. On the other hand a marked nucleating effect was reported to increase $\mathrm{T}_{\mathrm{C}}$ in composites obtained by an interfacial reaction between both components 
Table 4

Change in the crystallization peak temperature $\left(\Delta T_{c}\right)$, crystallinity $\left(\Delta \chi_{c}\right)$ and melting temperature $\left(\Delta T_{\llcorner}\right)$for different iPP nanocomposites.

\begin{tabular}{|c|c|c|c|c|c|c|c|}
\hline Nanofiller type & Nanofiller size $(\mathrm{nm})$ & Nanofiller content (\%) & Characteristics & $\Delta T_{\mathrm{c}}\left({ }^{\circ} \mathrm{C}\right)$ & $\Delta x_{c}(\%)$ & $\Delta T_{\mathrm{m}}\left({ }^{\circ} \mathrm{C}\right)$ & Ref. \\
\hline $\mathrm{Si}_{3} \mathrm{~N}_{4}$ & $60-80$ & $0.1-2$ & - & 3.4 & - & - & [124] \\
\hline $\mathrm{Si}_{3} \mathrm{~N}_{4}$ & $20 \leq$ & $\begin{array}{l}1 \\
2 \\
3 \\
4 \\
5\end{array}$ & - & $\begin{array}{l}0.3 \\
4.1 \\
0.9 \\
0.8 \\
1.3\end{array}$ & $\begin{array}{l}- \\
- \\
- \\
- \\
-\end{array}$ & $\begin{array}{l}- \\
- \\
- \\
-\end{array}$ & [209] \\
\hline $\mathrm{Si}_{3} \mathrm{~N}_{4}$ & $15-30$ & $\begin{array}{l}1 \\
2\end{array}$ & - & $\begin{array}{l}3 \\
5\end{array}$ & $\begin{array}{l}9.6 \\
3.8\end{array}$ & $\begin{array}{l}0 \\
2\end{array}$ & [208] \\
\hline $\mathrm{TiO}_{2}$ & 50 & $\begin{array}{l}5 \\
10 \\
20 \\
30 \\
5 \\
10 \\
20 \\
5 \\
10 \\
20 \\
30\end{array}$ & $\begin{array}{l}- \\
\mathrm{SiO}_{2} \text {-coated } \\
\mathrm{TiO}_{2} \\
\mathrm{SA}_{\text {-coated } \mathrm{TiO}_{2}} \\
-\end{array}$ & $\begin{array}{c}1.9 \\
1.8 \\
2.3 \\
2.2 \\
5.2 \\
0.9 \\
7.6 \\
-1 \\
0.2 \\
1.6 \\
0.4\end{array}$ & $\begin{array}{l}- \\
- \\
- \\
- \\
- \\
- \\
- \\
- \\
- \\
- \\
-\end{array}$ & $\begin{array}{l}- \\
- \\
- \\
- \\
- \\
- \\
- \\
- \\
- \\
- \\
-\end{array}$ & [207] \\
\hline Zno & $30-40$ & $\begin{array}{l}2 \\
0.3 \\
0.3\end{array}$ & $\begin{array}{l}- \\
\text { Organo } \mathrm{CH}_{3} \text {-coated } \mathrm{TiO}_{2} \\
\text { Organo F-coated } \mathrm{TiO}_{2}\end{array}$ & $\begin{array}{r}3 \\
14 \\
3\end{array}$ & $\begin{array}{l}- \\
- \\
-\end{array}$ & $\begin{array}{l}- \\
- \\
-\end{array}$ & [127] \\
\hline Zno & - & $\begin{array}{l}1.5 \\
3 \\
5 \\
1.5 \\
3 \\
5\end{array}$ & $\begin{array}{l}\text { Organosilane No irradiated C } \\
\text { Organosilane Irradiated C }\end{array}$ & $\begin{array}{l}1.3 \\
2.2 \\
3 \\
2.3 \\
2.4 \\
2.6\end{array}$ & $\begin{array}{r}0 \\
1.2 \\
2.4 \\
-4.5 \\
-6.2 \\
-6.7\end{array}$ & $\begin{array}{r}0.1 \\
-0.4 \\
0.3 \\
3.4 \\
3.3 \\
3\end{array}$ & [128] \\
\hline $\mathrm{ZnO}$ & $10-30$ & $\begin{array}{l}1 \\
3 \\
5 \\
15\end{array}$ & Dispersant agent & $\begin{array}{l}1.2 \\
2.6 \\
3.5 \\
3.9\end{array}$ & $\begin{array}{l}- \\
- \\
- \\
-\end{array}$ & $\begin{array}{l}- \\
- \\
- \\
-\end{array}$ & [129] \\
\hline ZnO & $50-70$ & $\begin{array}{l}2 \\
5 \\
7.5\end{array}$ & Biaxially oriented PP film & $\begin{array}{l}- \\
- \\
-\end{array}$ & $\begin{array}{r}-3 \\
-9 \\
-10\end{array}$ & $\begin{array}{l}-2 \\
-5 \\
-5\end{array}$ & [130] \\
\hline $\mathrm{Al}_{2} \mathrm{O}_{3}$ & 47 & $\begin{array}{l}1.5 \\
3 \\
5\end{array}$ & - & $\begin{array}{l}1.2 \\
3 \\
3.7\end{array}$ & $\begin{array}{l}0.6 \\
3.1 \\
2.3\end{array}$ & $\begin{array}{l}-1.5 \\
-1.3 \\
-0.6\end{array}$ & [134] \\
\hline $\mathrm{C}_{60}$ & & 2.4 & - & 2 & -7.8 & - & [142] \\
\hline $\mathrm{C}_{60}$ & & $\begin{array}{l}1.2 \\
1.2 / 0.1 \\
1.2 / 0.3\end{array}$ & $\begin{array}{l}- \\
\text { DHBP } \\
\text { DHBP }\end{array}$ & $\begin{array}{r}2.2 \\
13.5 \\
12.4\end{array}$ & $\begin{array}{l}- \\
- \\
-\end{array}$ & $\begin{array}{l}- \\
- \\
-\end{array}$ & [143] \\
\hline
\end{tabular}

Organo $\mathrm{CH}_{3}$ : Nucleating agent: 1,3,5-benzenetricarboxylic-( $\mathrm{N}$-2-methylcyclohexyl) triamine;Organo F: Nucleating agent: 1,3,5-benzenetricarboxylic-( $\mathrm{N}$ 4-fluorphenyl) triamine; DHBP: 2,5-dimethyl-2,5(terct-butylperoxy)hexane.

in the presence of DHBP [143] or DCP [142], where $\mathrm{C}_{60}$ is located in the centre of branched structures that provoke a nucleation effect at low $\mathrm{C}_{60}$ concentration [210], which subsequently diminishes due to the formation of crosslinked structures that reduce molecular mobility and also affect the crystallinity, without modification of the monoclinic crystalline structure of iPP [142]. In Table 4 some of the crystallization parameters of the aforementioned iPP/Si $\mathrm{N}_{4}$, iPP/TiO, , iPP/ZnO, iPP/Al $\mathrm{O}_{3}$ and $\mathrm{iPP} / \mathrm{C}_{60}$ nanocomposites are presented.

\subsubsection{Nylon-6}

Fig. 23a shows the DSC thermograms obtained during the crystallization from the melt and subsequent heating of nylon- 6 and its nanocomposites prepared with IF-WS 2 nanoparticles of around $80 \mathrm{~nm}$ [167]. The nucleating effect of the nanoparticles dispersed in the PA-6 matrix generated a upwards shift with concentration of around $4^{\circ} \mathrm{C}$ in $T_{\mathrm{C}}$ without variation in $\chi_{c}$ values of $34-36 \%$. Under isothermal conditions [144] this nucleating effect was confirmed as a function of crystallization temperature, Fig. 23b, where $\tau_{0.1}$ diminished, i.e. the overall crystallization rate increased, with increasing concentration of IF-WS 2 nanoparticles for the same crystallization temperature. At a $T_{\mathrm{C}}$ of $206^{\circ} \mathrm{C}$ the rate constant is $7.49 \times 10^{-7} \mathrm{~min}^{-1}$ for PA-6 and increases to $2.57 \times 10^{-2} \mathrm{~min}^{-1}$ for a content of $2 \mathrm{wt} . \% \mathrm{IF}^{-W S_{2}}$, being directly related to a reduction in the basal interfacial free energy associated with chain folding in the PA-6 crystal, $73.0 \mathrm{erg} \mathrm{cm}^{-2}$, compared to that corresponding to the crystal grown in the presence of 2 wt.\% nanoparticles, 

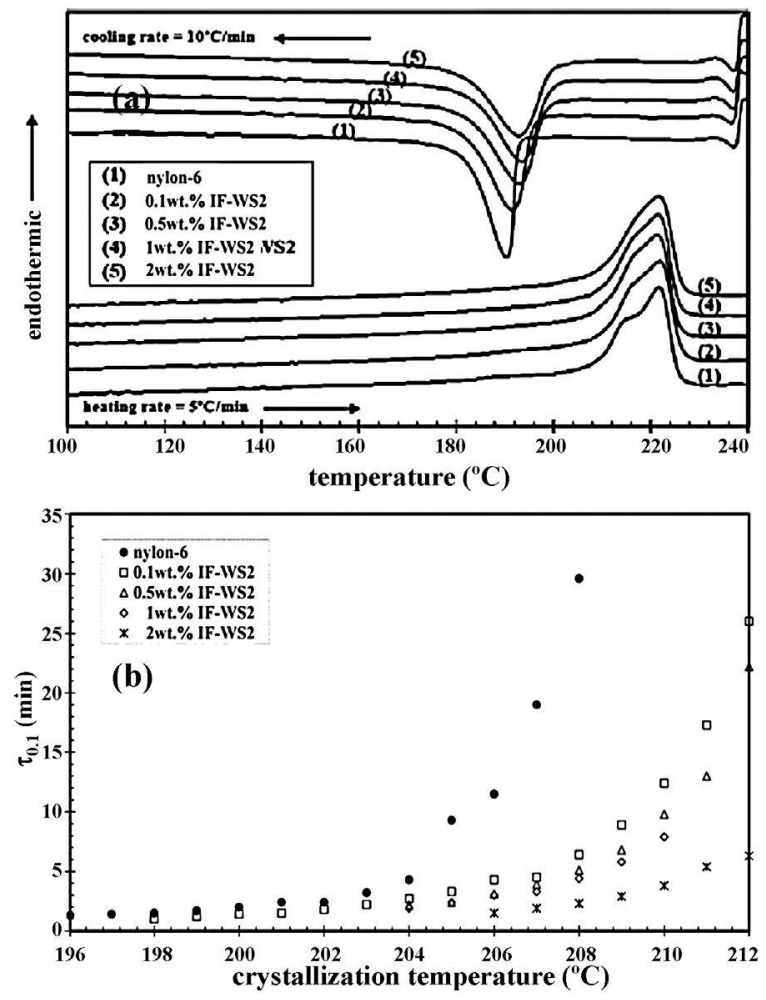

Fig. 23. (a) DSC thermograms of nylon-6/IF-WS 2 nanocomposites obtained during the cooling and heating rates indicated [167]; (b) time required to attain a $10 \%$ degree of transformation $\left(\tau_{0.1}\right)$ of nylon-6/IF-WS nanocomposites as a function of the crystallization temperature[144]. Reprinted with permission [167]. Copyright 2011, Elsevier; [144] Reprinted with permission. Copyright 2011, Elsevier.

$35.8 \mathrm{erg} \mathrm{cm}^{-2}$, and the consequent reduction in the free energy associated with the nucleation stage of PA-6 in the presence of the IF-WS 2 nanoparticles.

Nylon-6 can crystallize in at least two crystalline forms, denominated $\alpha$ and $\gamma$ [211-213] The more stable $\alpha$-phase at room temperature has characteristic peaks at $2 \theta \approx 21^{\circ}$ and $2 \theta \approx 24^{\circ}$ indexed as the 200 and $002 / 202$ reflections of the monoclinic unit cell, respectively. In the metastable $\gamma$-phase corresponding to a pseudo-hexagonal unit cell these peaks appear at $2 \theta \approx 22^{\circ}$ and $2 \theta \approx 23^{\circ}$, indexed as 001 and $200 / 201$, respectively, where the $200 / 201$ reflections appear as a shoulder to the 001 reflection. Further, the 020 reflection is weak in the $\alpha$-phase and intense in the $\gamma$-phase. In order to form hydrogen bonds between parallel chains in the pseudo-hexagonal $\gamma$-form crystals, the molecules need to twist away from the zigzag planes provoking lower inter-chain interaction compared to that in the monoclinic $\alpha$-form, where an all-trans configuration packs into a more stable antiparallel-chain arrangement of hydrogen bonds. Murthy et al. [213] observed that the room temperature monoclinic $\alpha$-form of nylon- 6 transforms into a high-temperature monoclinic structure on heating from room temperature, considered as the Brill transition. These complex crystal habits depend on varios factors including crystallization temperature, molecular weight, thermomechanical history and cooling conditions [213-215].
From wide angle $x$-ray diffraction (WAXS) of PA-6/IF$\mathrm{WS}_{2}$ nanocomposites [167] the crystallization of nylon from the molten state occurs firstly into a pseudohexagonal phase $(\gamma)$ and secondly into the monoclinic form $(\alpha)$, shown by the characteristic peaks at $2 \theta \approx 21^{\circ}$ and $2 \theta \approx 24^{\circ}$, Fig. 24 a. Where the nanocomposites crystallize at a slightly faster rate than nylon, once superceded the initial energy barrier to nucleation, the increased fraction of parallel chain-chain conformations may promote at higher temperature the growth of the more-disordered $\gamma$ phase. The reversible crystal-to-crystal Brill phase transition, i.e. the transformation of the $\alpha$ to $\gamma$-phase prior to melting, is typically observed when nylons are heated from room temperature [216] and is illustrated in Fig. 24b where the two $\alpha$-crystal phase peaks observed in the diffractograms of both PA-6 and its nanocomposite converge with increasing temperature and merge into a single asymmetric reflection at $2 \theta \approx 22^{\circ}$ characteristic of the $\gamma$-phase at the Brill transition, at $170-210^{\circ} \mathrm{C}$ for PA- 6 and $185-215^{\circ} \mathrm{C}$ for the $1 \mathrm{wt} . \%$ IF-WS 2 nanocomposite.

Shen et al. [145] have described a reduction in both $T_{\mathrm{c}}$ and $\chi_{\mathrm{c}}$ in $\mathrm{PA}-6 / \mathrm{SiO}_{2}$ nanocomposites with a concentration of 5.0 and 10 wt.\% using APTES, and more recently Cai et al. [149] also described the formation of the monoclinic polymorph in this type of nanocomposite with $\mathrm{SiO}_{2}$, although an increase of around $8 \%$ was observed in $\chi_{c}$ between that of neat nylon 6 and a nanocomposite with $5 \mathrm{wt} \%$ composition en $\mathrm{SiO}_{2}$. In nanocomposites of PA-6 with $\mathrm{CaCO}_{3}$ nanoparticles of $70 \mathrm{~nm}$ a reduction in both $T_{\mathrm{g}}$ and $\chi_{\mathrm{c}}$ of the polyamide was observed [175]. Rangari et al. [217] did not encounter any variations in crystallinity in PA-6 nanocomposites with spherical $\mathrm{Si}_{3} \mathrm{~N}_{4}$ particles.

\subsubsection{Poly(ether ether ketone)}

The incorporation into a PEEK matrix of concentrations as low as $0.1 \mathrm{wt} . \%$ of IF-WS 2 [57] leads to an increase of around $5^{\circ} \mathrm{C}$ in $T_{\mathrm{C}}$ of the matrix, Fig. 25, indicating that nucleation of PEEK crystals appears to be favored, and the crystalline structure of PEEK remains unchanged. However, further addition of IF-WS 2 caused a significant change in the behavior from promotion to retardation of crystallization although no variation was observed in $\chi_{c}$ of around $43 \%$. Further, it was calculated that the nucleation activity of PEEK falls on by the addition of nanoparticle concentrations under $0.5 \mathrm{wt} . \%$ confirming that the IF-WS 2 act effectively as NAs, whereas increasing the amount of IF-WS 2 leads to increased nucleation activity achieving values close to unity. This would suggest that the IF-WS 2 nanoparticles may impede the diffusion and rearrangement of the long polymer chains, thereby postponing the overall crystallization process.

The dynamic crystallization and melting behavior of PEEK in its nanocomposites with various inorganic nanoparticles has been discussed in some detail elsewhere [62]. For comparative purposes some of the salient features of PEEK nanocomposites with $\mathrm{Al}_{2} \mathrm{O}_{3}, \mathrm{Si}_{3} \mathrm{~N}_{4}$ and silica nanoparticles are given below, including several crystalline parameters presented in Table 5.

For $\mathrm{PEEK} / \mathrm{Al}_{2} \mathrm{O}_{3}$ nanocomposites with nanofiller contents between 0.8 and 10 wt.\%, Table 5, Kuo et al. 

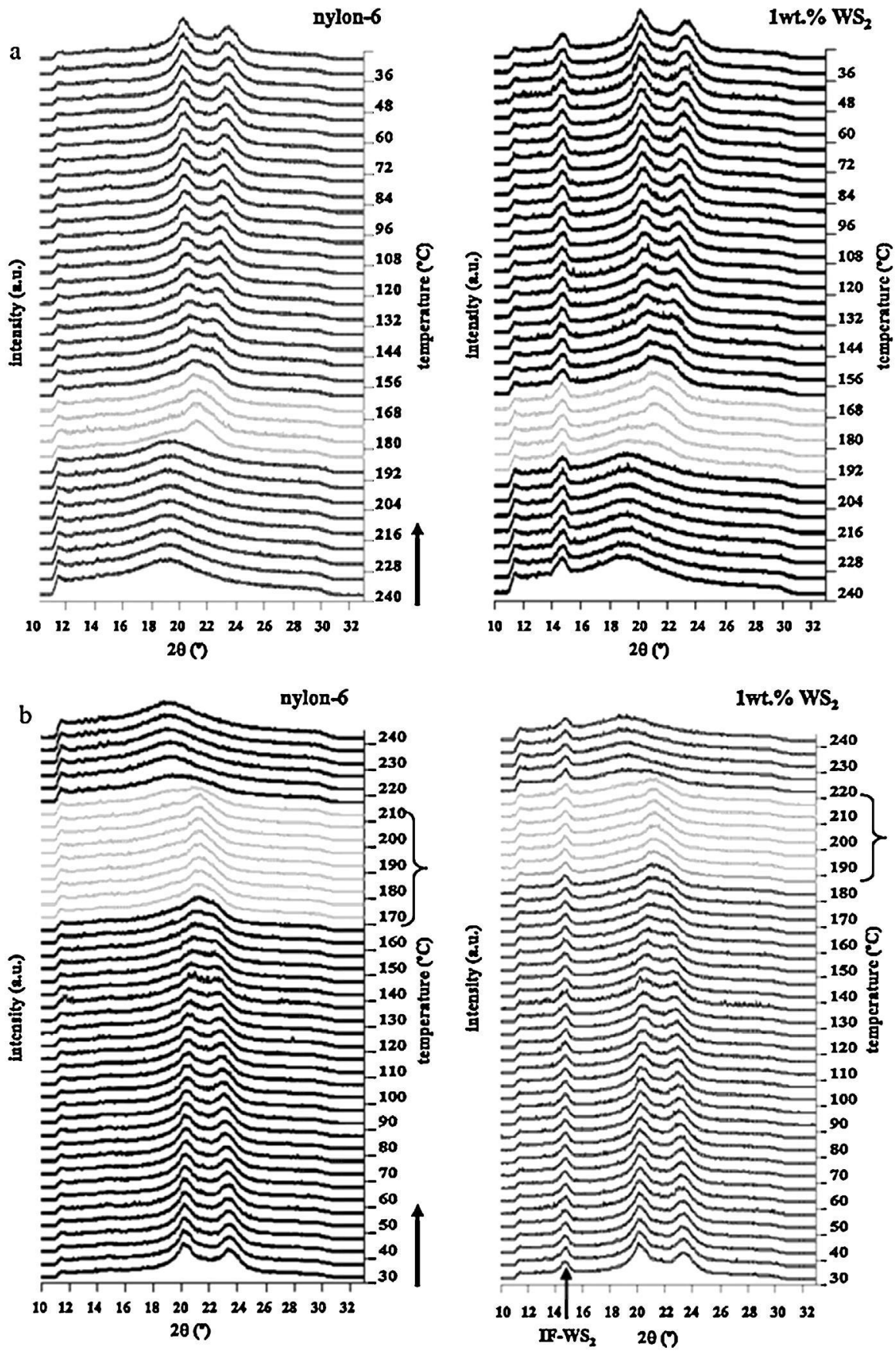

Fig. 24. WAXS diffractograms obtained during (a) cooling at $10^{\circ} \mathrm{C} / \mathrm{min}$ and (b) subsequent heating at $5^{\circ} \mathrm{C} / \mathrm{min}$ for nylon-6 and nylon-6/IF-WS 1 wt.\% nanocomposite.

Reprinted with permission [167]. Copyright 2011, Elsevier.

$[151,152]$ demonstrated a systematically lower $T_{c}$ for PEEK in the nanocomposite compared to that of neat PEEK, the decrease being most significant at the lowest nanoparticle concentration, suggesting substantial hindering of polymer chain mobility. Although heterogeneous nucleation increases on increasing the nanoparticle concentration up to $5 \mathrm{wt} . \%$, its effect less important. In contrast, Goyal et al. [150] recently described an increase in the $T_{\mathrm{C}}$ of PEEK by between 2 and $12^{\circ} \mathrm{C}$ on increasing the $\mathrm{Al}_{2} \mathrm{O}_{3}$ concentration from 2 to $30 \mathrm{wt} \%$, suggesting that the nucleating effect overrides the restrictions on molecular mobility, thus increasing the crystallization rate. The shape, size and surface morphology of the nanoparticles as well as to their degree of dispersion and adhesion with the matrix, combined with the preparative method used can explain these differences [62]. 
Table 5

Change in the crystallization peak temperature $\left(\Delta T_{\mathrm{c}}\right)$, crystallinity $\left(\Delta \chi_{\mathrm{c}}\right)$ and melting temperature $\left(\Delta T_{\mathrm{c}}\right)$ for different PEEK nanocomposites.

\begin{tabular}{|c|c|c|c|c|c|c|}
\hline Nanofiller type & Nanofiller size $(\mathrm{nm})$ & Nanofiller content (\%) & $\Delta T_{\mathrm{C}}\left({ }^{\circ} \mathrm{C}\right)$ & $\Delta X_{c}(\%)$ & $\Delta T_{\mathrm{m}}\left({ }^{\circ} \mathrm{C}\right)$ & Ref. \\
\hline \multirow[t]{7}{*}{$\mathrm{Al}_{2} \mathrm{O}_{3}$} & \multirow[t]{7}{*}{39} & 1 & -2 & -1.2 & 1 & \multirow[t]{7}{*}[150]{} \\
\hline & & 2 & 6 & -3.7 & 2 & \\
\hline & & 5 & 2 & -2 & 1 & \\
\hline & & 7 & 5 & -1.1 & 4 & \\
\hline & & 10 & 3 & -1.7 & 6 & \\
\hline & & 20 & 9 & -2.4 & 3 & \\
\hline & & 30 & 13 & 0.2 & 2 & \\
\hline \multirow[t]{4}{*}{$\mathrm{Al}_{2} \mathrm{O}_{3}$} & \multirow[t]{4}{*}{30} & 0.8 & -19 & -1.6 & -2 & \multirow[t]{4}{*}[151]{} \\
\hline & & 1.6 & -16 & -2.1 & -2 & \\
\hline & & 2.5 & -15 & -0.9 & -3 & \\
\hline & & 3.9 & -12 & -2.1 & -4 & \\
\hline \multirow[t]{4}{*}{$\mathrm{Al}_{2} \mathrm{O}_{3}$} & \multirow[t]{4}{*}{30} & 2.5 & -5 & 13.7 & 0 & \multirow[t]{4}{*}[152]{} \\
\hline & & 5 & -12 & 9.4 & 0 & \\
\hline & & 7.5 & -12 & 6.7 & -1 & \\
\hline & & 10 & -12 & 7.9 & -2 & \\
\hline \multirow[t]{5}{*}{$\mathrm{Si}_{3} \mathrm{~N}_{4}$} & \multirow[t]{5}{*}{50} & 2 & 8.2 & 3 & 1.5 & \multirow[t]{5}{*}[179]{} \\
\hline & & 5 & 11.9 & -1.8 & 2.9 & \\
\hline & & 10 & 14.2 & -4.4 & 1.5 & \\
\hline & & 20 & 0.5 & -6.8 & -0.2 & \\
\hline & & 30 & -4.5 & -11.7 & -2.1 & \\
\hline \multirow[t]{2}{*}{$\mathrm{SiO}_{2}$} & \multirow[t]{2}{*}{15} & 2.5 & -3 & -0.6 & 0 & \multirow[t]{2}{*}[155]{} \\
\hline & & 5 & -4 & -0.8 & 2 & \\
\hline \multirow[t]{4}{*}{$\mathrm{SiO}_{2}$} & \multirow[t]{4}{*}{30} & 2.5 & 2 & 4.4 & 0 & \multirow[t]{4}{*}{ [159] } \\
\hline & & 5 & 1 & -4.3 & 0 & \\
\hline & & 7.5 & 0 & -5.1 & 0 & \\
\hline & & 10 & -3 & -4.4 & 2 & \\
\hline \multirow[t]{4}{*}{$\mathrm{SiO}_{2}$} & \multirow[t]{4}{*}{15} & 2.5 & -4 & -5.2 & 1 & \multirow[t]{4}{*}{ [152] } \\
\hline & & 5 & -5 & 12.7 & -1 & \\
\hline & & 7.5 & -5 & 6.1 & -1 & \\
\hline & & 10 & -6 & 0.5 & -1 & \\
\hline \multirow[t]{4}{*}{$\mathrm{m}-\mathrm{SiO}_{2}{ }^{\mathrm{a}}$} & \multirow[t]{4}{*}{30} & 2.5 & -1 & -4 & 0 & \multirow[t]{4}{*}{ [159] } \\
\hline & & 5 & 1 & -3.1 & 2 & \\
\hline & & 7.5 & -2 & -2.5 & 0 & \\
\hline & & 10 & -2 & -4.7 & 2 & \\
\hline \multirow[t]{4}{*}{$\mathrm{SiO}_{2}{ }^{\mathrm{b}}$} & \multirow[t]{4}{*}{13} & 0.5 & 4 & 0.9 & -0.8 & [218] \\
\hline & & 1 & -2.4 & -1.1 & 0 & \\
\hline & & 2 & -2.6 & 2.2 & -1.3 & \\
\hline & & 4 & -3.8 & 0.8 & -2.6 & \\
\hline $\mathrm{SiO}_{2}{ }^{c}$ & 10 & 10 & -27.4 & -2.7 & 0.9 & [159] \\
\hline
\end{tabular}

a Surface modified with stearic acid.

b Premixed via ball milling.

c Modified with a silane coupling agent (GPTMS) and premixed via ball milling.

A homogeneous dispersion of the nanoparticles within the matrix was observed for PEEK nanocomposites containing $\mathrm{Si}_{3} \mathrm{~N}_{4}[153,179]$ and the $\mathrm{T}_{\mathrm{C}}$ of PEEK increases by around $14^{\circ} \mathrm{C}$ with $10 \mathrm{wt} . \%$ nanofiller due to heterogeneous nucleation. Up to $10 \mathrm{wt} \%$ loading the $T_{\mathrm{m}}$ of PEEK increases only very slightly and subsequently decreases at higher concentrations. On the other hand, the $\chi_{c}$ falls progressively with increasing $\mathrm{Si}_{3} \mathrm{~N}_{4}$ content in the $5-30 \mathrm{wt} . \%$ range. This suggests that a large number of nucleating centers impedes the crystallization of PEEK.

Finally, the incorporation of silica nanofillers into PEEK is by far the most extensively studied $[152,155,159,161,218]$. In all cases the $T_{c}$ of PEEK falls with increasing $\mathrm{SiO}_{2}$ concentration, and smaller crystalline lamellae develop that melt at lower temperatures compared to neat PEEK, Table 5. In general, silica nanoparticles have little influence on $\chi_{c}$ of PEEK with deviations of $<5 \%$.
In the case of surface modified silica, for example with SA [159], the values of $T_{C}$ observed were found to be slightly higher than the nanocomposite with the same amount of unmodified silica, but lower than that of neat PEEK, Table 5 , showing only a slight influence on molecular mobility with respect to the unmodified nanoparticle. In the case of silica-reinforced nanocomposites prepared by high energy ball milling followed by compression molding, Zhang et al. [218] described a heterogeneous nucleating effect at low nanoparticle concentration, Table 5, while Hedayati et al. [161] described a mechanism for the morphology evolution of the nanocomposites during milling explaining the decrease in both $T_{\mathrm{C}}$ and $\chi_{\mathrm{c}}$ due to restrictions on the molecular mobility of the polymer matrix during crystallization.

Crystallization kinetics studies of PEEK nanocomposites with inorganic nanoparticles are scarce, with examples 


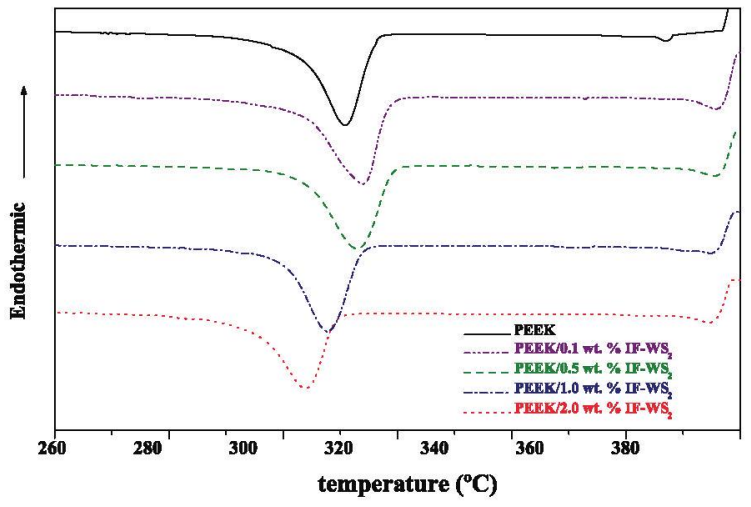

Fig. 25. Dynamic DSC crystallization thermograms for PEEK/IF-WS nanocomposites obtained by cooling from the melt to room temperature at a rate of $10^{\circ} \mathrm{C} \mathrm{min}^{-1}$.

Reprinted with permission [57]. Copyright 2010, American Chemical Society.

only for $\mathrm{Al}_{2} \mathrm{O}_{3}$ and $\mathrm{SiO}_{2}[151,155]$. As we have seen, athough the nanoparticles provide a substantial increase in the number of crystalline nuclei at a very high heterogeneous nucleation rate, the subsequent crystal growth is slow due to the hindered mobility of the crystallizable polymer chain segments, leading to smaller crystals and a reduced crystallization rate. This is illustrated in Fig. 26, where the overall crystallization rate constant, $k$ in the $T_{c}$ range between 260 and $310^{\circ} \mathrm{C}$, for neat PEEK and its 2.5 and 5 wt.\% nanocomposites with $15 \mathrm{~nm}$ nano- $\mathrm{SiO}_{2}$, falls with increased nanofiller loading [155]. A negative temperature coefficient indicates a slower crystallization rate with decreasing undercooling (i.e. increasing $T_{\mathrm{C}}$ ) and only at the highest $T_{C}$ values do the rate constants for neat PEEK and the nanocomposites approach similar values, since macromolecular transport becomes less important. It is clear that the nanoparticles affect both crystalline nucleation and transport, and the overall crystallization process in this type of system is more complex than the neat polymer, modifying the model of nucleation and crystalline growth [62].

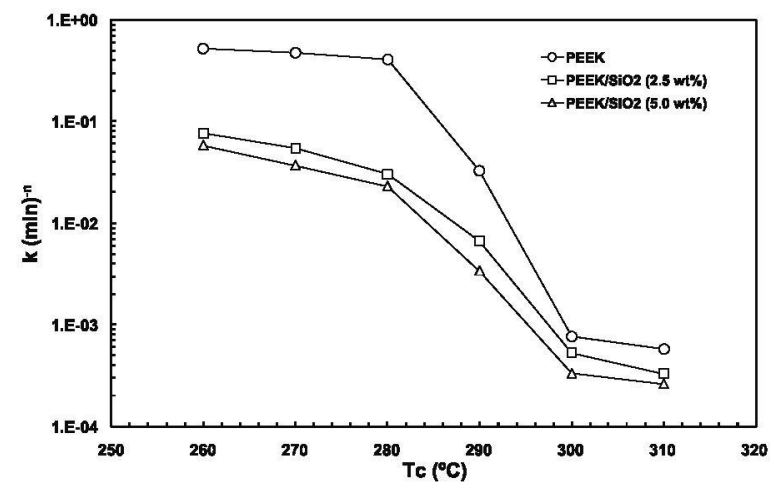

Fig. 26. Variation of the overall crystallization rate constant $(k)$ as a function of the crystallization temperature $\left(T_{c}\right)$ for PEEK and PEEK/SiO nanocomposites with different nanoparticle content. Extracted with permission [155]. Copyright 2010, Elsevier.
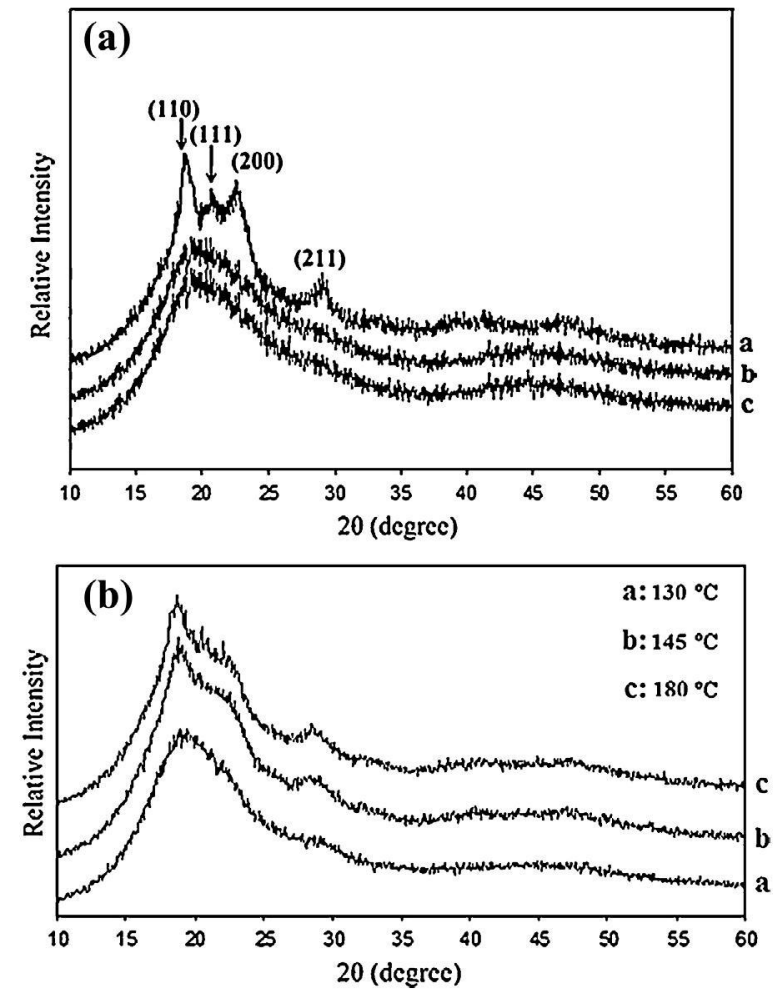

Fig. 27. X-ray diffraction patterns of (a) as-received PEEK, and ball-milled PEEK $/ \mathrm{SiO}_{2}$ powders after 2 and $5 \mathrm{~h}$, and (b) ball-milled PEEK/SiO $/ \mathrm{S}_{2}$ powders heat-treated at the temperatures indicated.

Reprinted with permission [161]. Copyright 2011, Elsevier.

With regard to the crystal structure, nanocomposites reinforced with inorganic nanoparticles crystallize in an orthorhombic unit cell, as in neat PEEK, but the intensity of the X-ray diffraction peaks is a function of nanofiller concentration, as observed in PEEK nanocomposites with $30 \mathrm{~nm} \mathrm{SiO}_{2}$ or $\mathrm{Al}_{2} \mathrm{O}_{3}$ nanoparticles [152]. No additional reflections or peak shifts were detected, indicating that the crystalline structure of the polymer was maintained and nanoparticle-matrix interactions were very weak. Occasionally, the crystalline reflections have been seen to broaden as the nanofiller content increases $[153,179]$ due to either a decrease in the crystallinity of PEEK and/or the formation of smaller, more imperfect crystals. In PEEK nanocomposites incorporating SA-modified silica [159], small shifts of the crystalline reflections to higher angles have been reported that suggest smaller interplanar crystalline distances, attributed to a more homogenous nanofiller dispersion with reduced distance between nanoparticles and increased concentration of crystals between them, resulting in spatial limitations during crystalline growth [178]. Shifts towards higher angles have also been described in $\mathrm{PEEK} / \mathrm{SiO}_{2}$ nanocomposites prepared by ball milling techniques [218], and in PEEK reinforced with nano- $\mathrm{SiO}_{2}$ modified with a GPTMS coupling agent [161]. In the latter case a progressive loss of PEEK crystals and a reduction in $\chi_{c}$ as a function of mixing time, Fig. 27a, and milling temperature, Fig. 27b, have been observed [161]. 


\subsubsection{Poly(phenylene sulphide)}

Attempts to circumvent disadvantages in the properties of PPS such as high fragility due to the difference between $T_{\mathrm{g}}$ and $T_{\mathrm{m}}$ have included the formation of composites with micro- and nano-scale fillers [163,164,219-225], including inorganic fullerenes of the type IF-WS $2[162,168,226]$. The cooling thermograms for PPS/IF-WS ${ }_{2}$ nanocomposites with nanoparticle contents from 0.05 to 8 wt.\% [98] are shown in Fig. 28 where a reduction in the $T_{\mathrm{C}}$ of PPS is observed on the addition of concetrations $\leq 0.1 \mathrm{wt} . \%$. This phenomenon occurs at cooling rates between 1 and $20^{\circ} \mathrm{Cmin}^{-1}$ and is completely unusual in the crystallization of nanoparticlefilled polymers at these concentration levels [98,227-229]. Increasing the IF-WS 2 content provokes a drastic change from lowering to increasing the crystallization temperature related to the nucleating activity of the IF particles, whilst maintaining the orthorrhombic structure of PPS [230].

On the other hand, PPS exhibits a cold crystallization process when heated above $T_{g}$, and the crystals formed on prior cooling from the melt are those that act as nucleating sites during the heating process, modifying the cold crystallization behavior. In Fig. 29a it can be observed that in nanocomposites with $>0.1 \mathrm{wt} . \%$ IF-WS 2 the cold crystallization temperature, $T_{\mathrm{cc}}$ falls, indicating a nucleating effect, whilst $T_{\text {cc }}$ increases at lower nanoparticle concentrations [168]. PPS nanocomposites with low IF-WS 2 concentrations, $\leq 0.1 \mathrm{wt} \%$, showed higher crystallization activation energy values than neat PPS, Fig. 29b, implying higher impediments for the transport of the macromolecular segments to the surface of the growing PPS crystal and, as a consequence the crystallization process is delayed. An increase in the nanofiller concentration leads to an decrease in the activation energy, implying easier crystal formation due to the nucleating activity of IF-WS 2 . By comparison, the overall crystallization rate constant, $k$ fell from $1.0 \times 10^{-4}$ for neat PPS to $4.5 \times 10^{-6}$ for $0.1 \mathrm{wt} . \%$ IF$\mathrm{WS}_{2}$, increasing to $2.1 \times 10^{-2}$ for $2 \mathrm{wt}$ \% nanofiller. This phenomenon of reduction and subsequent increment in the crystallization rate as a function of IF concentration is associated with the variation of the basal interfacial free energy, $\sigma_{\mathrm{e}}$ directamente related to the folding energy of the basal planes of the crystal that evolve from $81 \mathrm{erg} \mathrm{cm}^{-2}$ for neat PPS to $89 \mathrm{erg} \mathrm{cm}^{-2}$ for the nanocomposite with $0.1 \mathrm{wt} . \%$ IF-WS 2 , falling to $41 \mathrm{erg} \mathrm{cm}^{-2}$ for the $2 \mathrm{wt} . \%$ nanocomposite. This implies, firstly an increase in the free energy of nucleation and subsequently a decrease that, added to the variations in the activation energy related to molecular transport, justifies the deceleration and acceleration effects on the crystallization rate of PPS [162].

Very little information is available on the crystallization behavior of PPS with pseudospherical nanoparticles. Lu et al. $[163,164]$ have studied nanocomposites with $\mathrm{SiO}_{x}$ nanofillers, where $x=1.2-1.6$, with sizes between 5 and $15 \mathrm{~nm}$ and SSA between 640 and $700 \mathrm{~m}^{2} \mathrm{~g}^{-1}$, prepared from the melt using surface grafted epoxy coupling agents. They used different preparation methodologies fundamentally based on thermal pre-treatment of PPS and pre-blending by ball milling, detailed in the foot of Table 6 where values of the crystallization parameters of PPS are given as a function of the preparative method. Thermal pre-treatment of PPS at $260^{\circ} \mathrm{C}$ for $5 \mathrm{~h}$, HTM-PPS, increases the degree of crosslinking in the PPS, caused by the thioether bond that forms at elevated temperatures, which is pre-treatment time and temperature dependent [231]. Increasing the degree of crosslinking leads to a reduction in both $\chi_{\mathrm{c}}$ and $T_{\mathrm{c}}$ as a consequence of the reduced molecular mobility of the polymer chain segments, affecting the crystallization of PPS to a higher degree than the blending process at $292^{\circ} \mathrm{C}$. The presence of silica nanoparticles has practically no effect on the crystalline parameters of PPS in the nanocomposites processed without thermal pre-treatment, NM-PPS, but a slight nucleating effect was observed by an approximately $5^{\circ} \mathrm{C}$ increase in $T_{\mathrm{c}}$ in the nanocomposites with thermal pre-treatment, NHTM-PPS [163]. The incorporation of a pre-blending step via ball milling to the thermal pre-treatment of PPS slightly modifies the crystalline parameters of the matrix in the silica nanocomposites, especially when applied immediately afterwards, FHTM12h-PPS, obtaining nanocomposites with the lowest $\chi_{\mathrm{c}}$. This fact appears to be a consequence of the possible insertion of silica nanoparticles in the crosslinked network generated in the PPS matrix by heating at $260^{\circ} \mathrm{C}$ for $28 \mathrm{~min}$ [164] (Table 6).

\subsubsection{Polymethylene oxide}

Nanoparticles of IF-MoS 2 with an average diameter of around $150 \mathrm{~nm}$ show a very slight nucleation effect in Polymethylene oxide (POM), of around $5^{\circ} \mathrm{C}$ up to a concentration of $2 \%$, in melt processed POM/IF-MoS 2 nanocomposites, which is accompanied by an increase of around $3 \%$ in $\chi_{c}$, but only at concentrations up to $1.5 \%$. Above this, a sharp decrease in $\chi_{c}$ from $55 \%$ to $49 \%$ was described as a consequence of the inhibition in the formation of crystalline order by the nanoparticles [165], Fig. 30.

\subsection{Thermal conductivity}

Most polymers have a thermal conductivity $(\lambda)$ in the range of $0.1-0.5 \mathrm{~W} / \mathrm{mK}$ that limits their use in industrial applications. To overcome this limitation, different inorganic fillers such as AIN $\left(\lambda \sim 200 \mathrm{~W} \mathrm{~m}^{-1} \mathrm{~K}^{-1}\right)$, BN $\left(\lambda \sim 250 \mathrm{Wm}^{-1} \mathrm{~K}^{-1}\right), \mathrm{Al}_{2} \mathrm{O}_{3}\left(\lambda \sim 20 \mathrm{Wm}^{-1} \mathrm{~K}^{-1}\right)$, BeO $\left(\lambda \sim 260 \mathrm{~W} \mathrm{~m}^{-1} \mathrm{~K}^{-1}\right)$, etc. [232] have been incorporated into polymeric matrices. The thermal conductivity of these composites is strongly influenced by the filler packing density, particle size and distribution, surface treatment and processing methods [233-235]. Dispersion and orientation states leading to the formation of networks are known to enhance thermal conductivity [236], although percolation threshold behavior is barely observed. The interfacial thermal resistance, a barrier to the heat flow associated with the differences in the phonon spectra of the two phases, and the phonon scattering at the interface are crucial factors in nanocomposites [237]. Small nanoparticles lead to large interfacial areas and high interfacial thermal resistance, which cause elevated levels of phonon scattering, thereby resulting in lower $\lambda$ values than those observed for microparticles. Nanoparticle surface treatment improves the nanofiller-matrix interactions increasing thermal transport across the interface. 


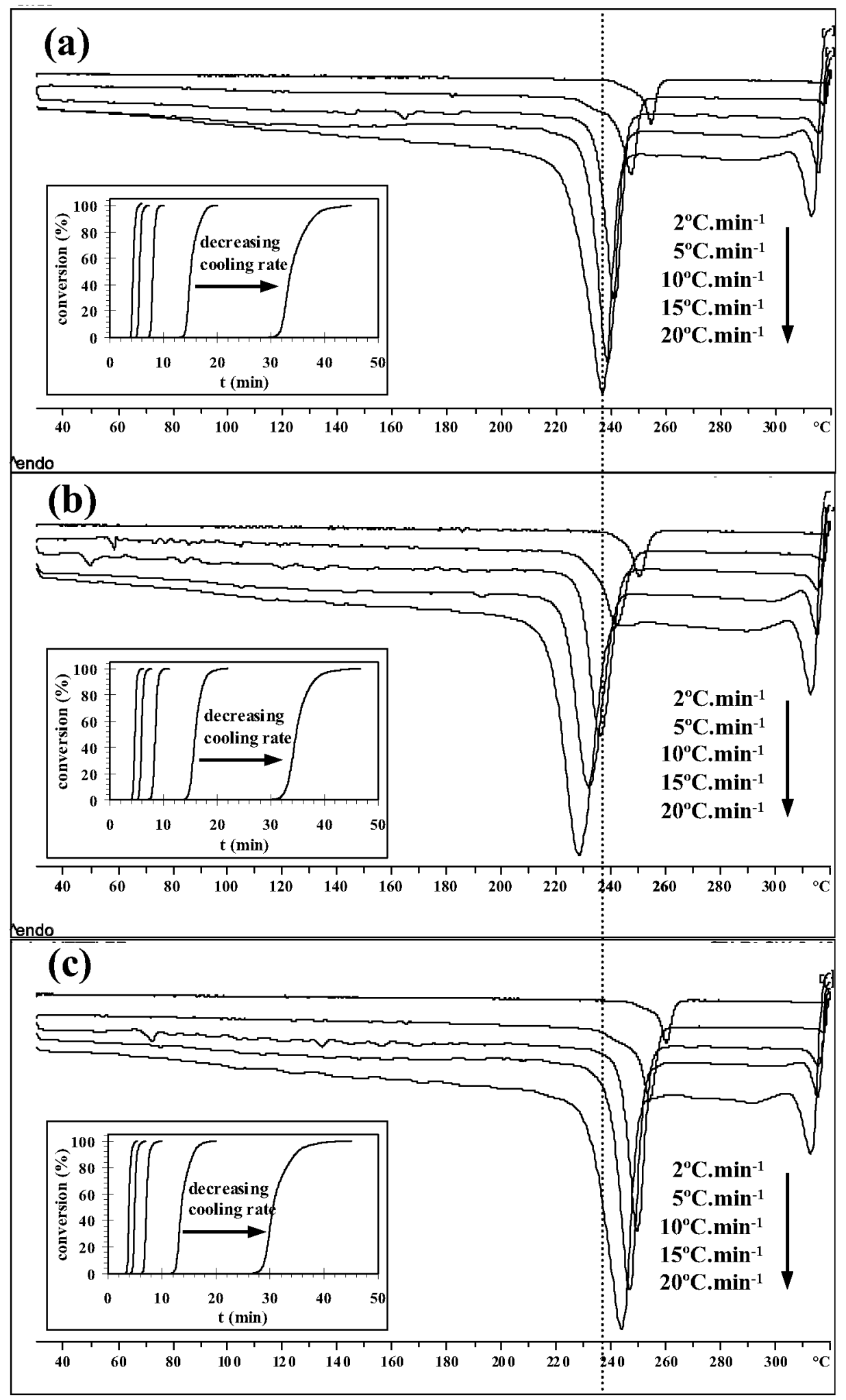

Fig. 28. DSC thermograms of dynamic crystallization of (a) neat PPS, (b) PPS/IF-WS 20.1 wt.\%, and (c) PPS/IF-WS 2 wt.\% obtained at various cooling rates. Reprinted with permission [226]. Copyright 2009, American Chemical Society. 


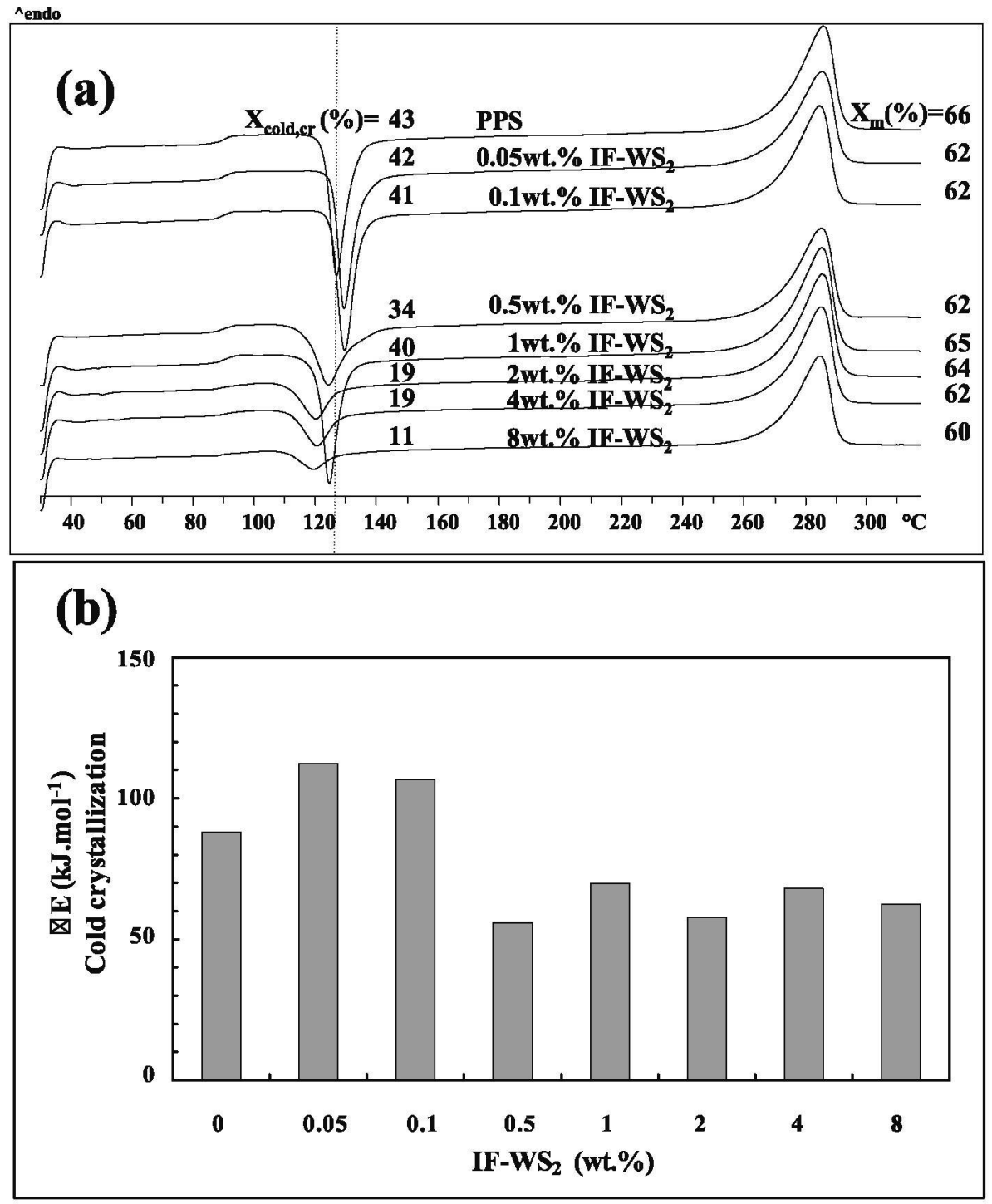

Fig. 29. (a) DSC thermograms of PPS/IF-WS 2 nanocomposites obtained during heating at $10^{\circ} \mathrm{Cmin}^{-1}$, and (b) variation in the energy of activation of the

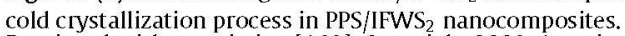

Reprinted with permission [168]. Copyright 2009, American Chemical Society.

Table 6

Effect of the heat treatment and ball milling on the crystal parameters of PPS in PPS/nano-SiOx.

\begin{tabular}{|c|c|c|c|c|c|}
\hline Sample & Nanofiller content $(\%)$ & $T_{\mathrm{c}}\left({ }^{\circ} \mathrm{C}\right)$ & $x_{c}(\%)$ & $T_{\mathrm{m}}\left({ }^{\circ} \mathrm{C}\right)$ & Ref. \\
\hline M-PPSa & 0 & 244.9 & 61 & 283.3 & {$[163,164]$} \\
\hline HTM-PPS $^{b}$ & 0 & 225.9 & 51.6 & 278.8 & \\
\hline NM-PPS & 3 & 243.3 & 61 & 283.4 & \\
\hline NHTM-PPS $^{d}$ & 3 & 231.1 & 47.9 & 277.3 & \\
\hline 12hM-HTM-PPS & 3 & 232.5 & 50.1 & 280 & \\
\hline FHTM-12hM-PPS ${ }^{f}$ & 3 & 228.4 & 48.9 & 277.9 & \\
\hline
\end{tabular}

a PPS mixed at $292^{\circ} \mathrm{C}$ for $0.28 \mathrm{~h}$.

b PPS heat treated at $260^{\circ} \mathrm{C}$ for $5 \mathrm{~h}$ and then mixed at $292^{\circ} \mathrm{C}$ for $0.28 \mathrm{~h}$.

c PPS with $3 \%$ nano-SiO $x$ mixed at $292^{\circ} \mathrm{C}$ for $0.28 \mathrm{~h}$.

${ }^{d}$ Heat treated PPS with $3 \%$ nano- $\mathrm{SiO}_{x}$ mixed at $292^{\circ} \mathrm{C}$ for $0.28 \mathrm{~h}$.

e PPS first milled with $\mathrm{SiO}_{x}$ for $12 \mathrm{~h}$ then heat-treated at $260^{\circ} \mathrm{C}$ for $5 \mathrm{~h}$ and finally mixed at $292^{\circ} \mathrm{C}$ for $0.28 \mathrm{~h}$.

f PPS first treated at $260^{\circ} \mathrm{C}$ for $5 \mathrm{~h}$ then milled with $\mathrm{SiO}_{\mathrm{x}}$ for $12 \mathrm{~h}$ and finally mixed at $292^{\circ} \mathrm{C}$ for $0.28 \mathrm{~h}$. 

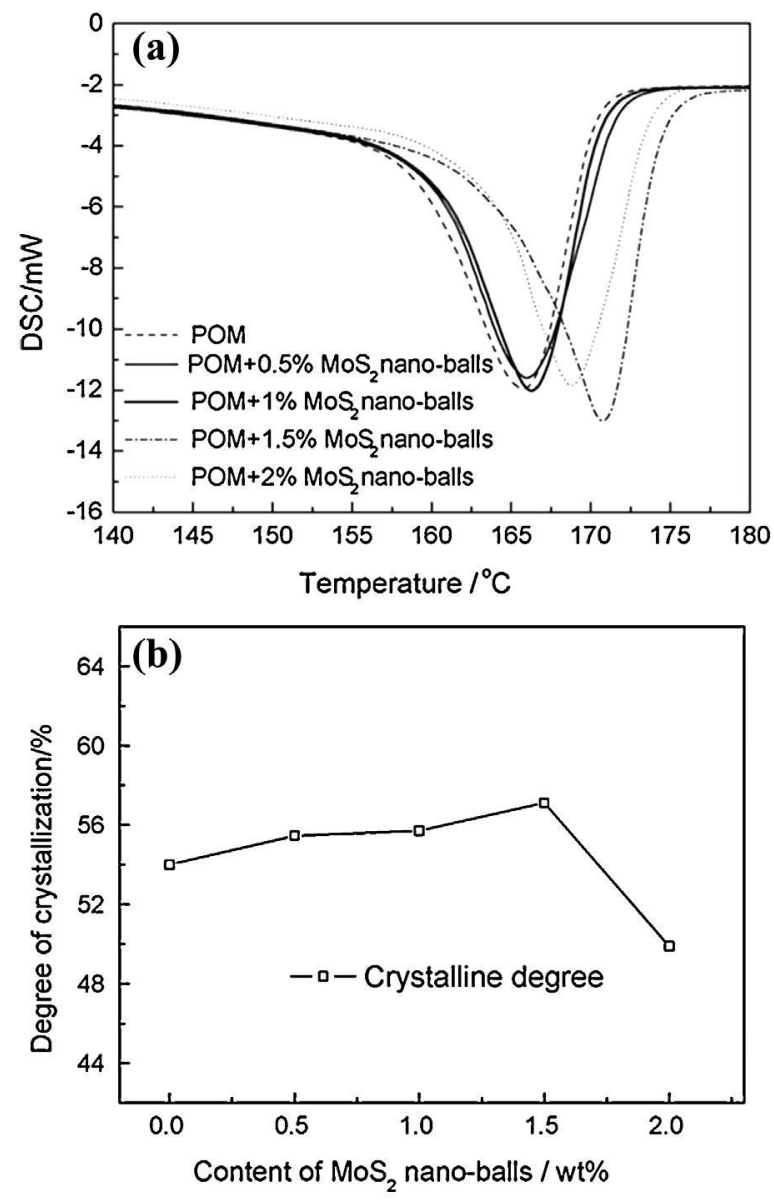

Fig. 30. (a) DSC curves for POM and POM with $\mathrm{MoS}_{2}$ nano-balls, and (b) the deduced crystallinity as a function of nanoparticle concentration. Reprinted with permission [165]. Copyright 2009, Elsevier.

In an attempt to improve the thermal conductivity of high-performance thermoplastics, small amounts of IF-WS 2 were incorporated into PEEK and PPS [57,238]. These nanoparticles display a $\lambda$ value of $\sim 0.5 \mathrm{~W} \mathrm{~m}^{-1} \mathrm{~K}^{-1}$ [239], comparable to that reported for nanoclays [240], and higher than those of the neat polymers $(0.26$ and $0.21 \mathrm{~W} \mathrm{~m}^{-1} \mathrm{~K}^{-1}$, respectively [241]). Naffakh et al. [57] investigated the thermal conductivity of PEEK/IF-WS (0.1-2 wt.\%) nanocomposites prepared by ultrasonication and melt-blending processes using a steady state method. $\lambda$ of the matrix was found to rise progressively with increasing loading, the increments being $\sim 10,27,41$, and $52 \%$ for nanocomposites with $0.1,0.5,1$ and 2 wt.\% IF-WS 2 , respectively. However, in composites where the sonication step was omitted the improvements were significantly lower due to the presence of agglomerates, corroborating that the thermal conductivity is sensitive not only to the filler concentration but also to the state of dispersion, identical to the case for PEEK/SWCNT nanocomposites [242]. The thermal conductivity of PPS was also remarkably enhanced by the addition of these nanoparticles with increments in $\lambda$ of $8,20,36$ and $46 \%$, for nanocomposites with $0.1,0.5,1.0$ and $2 \mathrm{wt} . \%$ IF-WS 2 , respectively. The improvement in the thermal conductivity of these thermoplastics upon addition of the IF-WS $\mathrm{W}_{2}$ is useful for the reduction of cycle times, hence increasing processing efficiency of these polymers at industrial level when techniques like injection molding are used.

A wide number of theoretical and empirical models have been proposed to estimate the thermal conductivity of binary composites. Maxwell [243] developed a simple equation to predict $\lambda$ of randomly and individually dispersed spheres in a homogeneous medium. The effective thermal conductivity of nanocomposites with very low nanoparticle volume fraction can be written as: $\lambda_{c /} \lambda_{m}=1+3\left[\left(\lambda_{\mathrm{f}}-\lambda_{m}\right) /\left(\lambda_{\mathrm{f}}+2 \lambda_{m}\right)\right]$, where $\lambda_{c}, \lambda_{m}$ and $\lambda_{\mathrm{f}}$ are the thermal conductivities of the composite, matrix and filler, respectively. Naffakh et al. [238] applied this model to predict the thermal conductivity of IF-WS 2 filled PEEK and PPS nanocomposites, and for samples reinforced with $1.0 \mathrm{wt} . \%$ loading $\lambda$ was calculated to be 0.45 and $0.41 \mathrm{~W} / \mathrm{mK}$, respectively, $\sim 25$ and $30 \%$ higher than the experimental values. The disparity between theoretical and measured data was mainly ascribed to heat dissipation across the nanoparticle-polymer interface due to imperfect thermal contact between the phases. Articles on the modeling of the thermal conductivity of polymer/IF-WS 2 nanocomposites are lacking, and further investigation in this area will be worthwhile.

\subsection{Mechanical properties}

One of the primary objectives of the addition of inorganic nanoparticles to polymers is to improve their mechanical performance. The nanoparticles exhibit higher SSA, surface energy and density compared to microparticles, and consequently, lower nanofiller concentrations (1-10\%) are required to obtain properties comparable to, or even better than, those achieved by conventional microfiller loadings (15-40\%). This facilitates processing and reduces the increase in composite weight. However, as was previously shown, the strong tendency for nanoparticles to agglomerate inhibits their efficient dispersion within the matrix, which can severely limit property enhancement. Moreover, a strong nanoparticlematrix interfacial adhesion is required to achieve effective load transfer that leads to good reinforcement. Thus, from an applications viewpoint it is crucial to find an appropriate balance between stiffness/strength and toughness.

The mechanical properties of nanocomposites are usually characterized through a variety of techniques, and include parameters such as Young's modulus, storage modulus, tensile strength, impact strength, and so forth, which will be discussed in detail in the following section. Tables $7-10$ collect the most relevant room temperature mechanical data published on thermoplastic/IF-WS 2 nanocomposites and compares them with data reported for nanocomposites incorporating other spherical or quasispherical inorganic nanofillers.

\subsubsection{Polypropylene}

In general, PP has good mechanical properties, although it presents some deficiencies such as low impact resistance. Due to its non-polar character, functionalization of the polymer and/or surface treatment of the nanoparticles are generally required to achieve a good nanofiller dispersion, 
Table 7

Comparison between the mechanical properties of polypropylene/IF-WS $S_{2}$ and other polypropylene/inorganic nanoparticle composites.

\begin{tabular}{|c|c|c|c|c|c|c|c|c|c|}
\hline $\begin{array}{l}\text { Nanofiller (Particle } \\
\text { size/nm) }\end{array}$ & $\begin{array}{l}\text { Nanofiller content } \\
\text { (wt.\%) }\end{array}$ & $\begin{array}{l}\text { Processing } \\
\text { method }\end{array}$ & $\Delta E(\%)$ & $\Delta \sigma_{y}(\%)$ & $\Delta E_{\mathrm{f}}(\%)$ & $\Delta \sigma_{\mathrm{fm}}(\%)$ & $\Delta E_{\text {charpy }}(\%)$ & $\Delta E^{\prime}(\%)$ & Ref. \\
\hline \multirow{4}{*}{ IF-WS $2(40-180)$} & 0.1 & $\mathrm{mb}$ & - & - & - & - & - & 7 & [93] \\
\hline & 1 & & 39 & 36 & 29 & 17 & -12 & 34 & \\
\hline & 4 & & - & - & - & - & - & 20 & \\
\hline & 8 & & - & - & - & - & - & 33 & \\
\hline \multirow[t]{4}{*}{$\mathrm{SiO}_{2}(<100)$} & 1 & $u l t+m b$ & - & -5 & - & - & 2 & - & [191] \\
\hline & 2 & & - & -7 & - & - & 6 & - & \\
\hline & 3 & & - & -8 & - & - & -1 & - & \\
\hline & 4 & & - & -17 & - & - & -4 & - & \\
\hline \multirow{5}{*}{$\begin{array}{l}\text { APTES-modified } \mathrm{SiO}_{2} \\
\quad(<100)\end{array}$} & 1 & $\mathrm{ult}+\mathrm{mb}$ & - & 2 & - & - & 25 & - & [191] \\
\hline & 2 & & - & 3 & - & - & 35 & - & \\
\hline & 3 & & - & 5 & - & - & 35 & - & \\
\hline & 4 & & - & 8 & - & - & 50 & - & \\
\hline & 5 & & - & 2 & - & - & 54 & - & \\
\hline $\mathrm{SiO}_{2}(12)$ & 5.3 & $\mathrm{mb}$ & 20 & -2 & - & - & - & 22 & [256] \\
\hline $\begin{array}{l}\text { HMDS-modified } \mathrm{SiO}_{2} \\
\text { (12) }\end{array}$ & 5.3 & $\mathrm{mb}$ & 15 & 0 & - & - & - & 26 & [256] \\
\hline $\mathrm{SiO}_{2}(10-20)$ & 4.5 & ext & 33 & -5 & - & - & 68 & - & [253] \\
\hline \multirow{5}{*}{$\begin{array}{l}\mathrm{PP}-\mathrm{g} \text {-MAH modified } \\
\mathrm{SiO}_{2}(7)\end{array}$} & 1 & $\mathrm{mb}$ & 9 & 9 & - & - & 43 & - & [114] \\
\hline & 2 & & 6 & 9 & - & - & 57 & - & \\
\hline & 3 & & 7 & 12 & - & - & 27 & - & \\
\hline & 4 & & 23 & 9 & - & - & 33 & - & \\
\hline & 5 & & 12 & 10 & - & - & -10 & - & \\
\hline \multirow{5}{*}{$\begin{array}{l}\text { PP-methyl-POSS } \\
\text { modified } \mathrm{SiO}_{2}(7)\end{array}$} & 1 & $\mathrm{mb}$ & 7 & 0 & - & - & 27 & - & [114] \\
\hline & 2 & & 6 & 2 & - & - & 37 & - & \\
\hline & 3 & & 5 & 0 & - & - & -50 & - & \\
\hline & 4 & & 15 & 6 & - & - & 30 & - & \\
\hline & 5 & & 13 & 7 & - & - & -60 & - & \\
\hline \multirow[t]{5}{*}{ Fumed $\mathrm{SiO}_{2}(12)$} & 1 & ext & 9 & 2 & - & - & 20 & - & [94] \\
\hline & 2.5 & & 13 & 3 & - & - & 23 & - & \\
\hline & 5 & & 18 & 2 & - & - & 19 & - & \\
\hline & 7.5 & & 24 & 2 & - & - & 15 & - & \\
\hline & 10 & & 25 & 1 & - & - & 12 & - & \\
\hline \multirow[t]{4}{*}{ PS-g-SiO ${ }_{2}(10)$} & 0.9 & isp & 4 & 4 & - & - & 42 & - & {$[110,254]$} \\
\hline & 1.8 & & 8 & 6 & - & - & 70 & - & \\
\hline & 3.7 & & 10 & 3 & - & - & 40 & - & \\
\hline & 7.6 & & 23 & 7 & - & - & 150 & - & \\
\hline PVA-g-SiO $2(10)$ & 7.6 & isp & 8 & 3 & - & - & 185 & - & [254] \\
\hline \multirow[t]{4}{*}{$\mathrm{PBA}-\mathrm{g}-\mathrm{SiO}_{2}(10)$} & 0.9 & isp & 0 & 6 & - & - & 85 & - & [112] \\
\hline & 1.8 & & 30 & 8 & - & - & 135 & - & \\
\hline & 2.5 & & 35 & 7 & - & - & 150 & - & \\
\hline & 4.4 & & 37 & 8 & - & - & 130 & - & \\
\hline \multirow[t]{5}{*}{$\mathrm{Al}_{2} \mathrm{O}_{3}(20-30)$} & 1 & $\mathrm{mb}$ & -18 & -12 & 7 & -1 & - & - & {$[247]$} \\
\hline & 2 & & -6 & -11 & 12 & -2 & - & - & \\
\hline & 3 & & 3 & -2 & 10 & -5 & - & - & \\
\hline & 4 & & 4 & 4 & 11 & -3 & - & - & \\
\hline & 5 & & 4 & 0 & 15 & -1 & - & - & \\
\hline \multirow[t]{5}{*}{$\mathrm{Al}_{2} \mathrm{O}_{3}(30-70)$} & 1 & $\mathrm{mb}$ & - & 6 & - & - & 9 & - & [248] \\
\hline & 3 & & - & 11 & - & - & 21 & - & \\
\hline & 5 & & - & 12 & - & - & 6 & - & \\
\hline & 10 & & - & 4 & - & - & 1 & - & \\
\hline & 15 & & - & 2 & - & - & -1 & - & \\
\hline \multirow[t]{4}{*}{$\mathrm{CaCO}_{3}(70-90)$} & 3 & ext & 44 & 13 & - & - & 30 & - & [251] \\
\hline & 5 & & 44 & 6 & - & - & 27 & - & \\
\hline & 7 & & 39 & 6 & - & - & 17 & - & \\
\hline & 10 & & 39 & 3 & - & - & 19 & - & \\
\hline
\end{tabular}


Table 7 (Continued)

\begin{tabular}{|c|c|c|c|c|c|c|c|c|c|}
\hline $\begin{array}{l}\text { Nanofiller (Particle } \\
\text { size/nm) }\end{array}$ & $\begin{array}{l}\text { Nanofiller content } \\
\text { (wt.\%) }\end{array}$ & $\begin{array}{l}\text { Processing } \\
\text { method }\end{array}$ & $\Delta E(\%)$ & $\Delta \sigma_{\mathrm{y}}(\%)$ & $\Delta E_{\mathrm{f}}(\%)$ & $\Delta \sigma_{\mathrm{fm}}(\%)$ & $\Delta E_{\text {charpy }}(\%)$ & $\Delta E^{\prime}(\%)$ & Ref. \\
\hline \multirow{6}{*}{$\mathrm{CaCO}_{3}(40)$} & 0.5 & ext & - & -2 & 6 & - & 24 & - & [249] \\
\hline & 1 & & - & 3 & 7 & - & 27 & - & \\
\hline & 2.5 & & - & 6 & 8 & - & 22 & - & \\
\hline & 5 & & - & 0 & 10 & - & 27 & - & \\
\hline & 10 & & - & -5 & 16 & - & 9 & - & \\
\hline & 20 & & - & -6 & 29 & - & 4 & - & \\
\hline \multirow[t]{3}{*}{$\mathrm{CaCO}_{3}(70)$} & 12.9 & ext & 46 & 3 & - & - & 8 & - & [255] \\
\hline & 23.8 & & 76 & 6 & - & - & 33 & - & \\
\hline & 41.3 & & 112 & 3 & - & - & 60 & - & \\
\hline \multirow[t]{3}{*}{$\mathrm{CaCO}_{3}(30-40)$} & 5 & ext & - & - & - & - & - & 0 & [117] \\
\hline & 10 & & - & - & - & - & - & 0 & \\
\hline & 15 & & - & - & - & - & - & 2 & \\
\hline \multirow{6}{*}{$\begin{array}{l}\text { MA-modified } \mathrm{CaCO}_{3} \\
(40)\end{array}$} & 0.5 & & - & 4 & 0 & - & 116 & - & [249] \\
\hline & 1 & ext & - & 13 & 2 & - & 129 & - & \\
\hline & 2.5 & & - & 13 & 2 & - & 190 & - & \\
\hline & 5 & & - & 5 & 5 & - & 120 & - & \\
\hline & 10 & & - & -9 & 7 & - & 96 & - & \\
\hline & 20 & & - & -6 & 13 & - & 30 & - & \\
\hline \multirow{4}{*}{$\begin{array}{l}\text { SA-modified } \mathrm{CaCO}_{3} \\
\quad(40)\end{array}$} & 10 & $\mathrm{mb}$ & - & - & - & - & - & 0 & [199] \\
\hline & 20 & & - & - & - & - & - & 7 & \\
\hline & 25 & & - & - & - & - & - & 50 & \\
\hline & 30 & & - & - & - & - & - & 57 & \\
\hline \multirow{3}{*}{$\begin{array}{l}\text { SA-modified } \mathrm{CaCO}_{3} \\
\quad(70)\end{array}$} & 12.9 & ext & 28 & 3 & - & - & 100 & - & [255] \\
\hline & 23.8 & & 30 & 5 & - & - & 550 & - & \\
\hline & 41.3 & & 28 & -2 & - & - & 300 & - & \\
\hline \multirow{4}{*}{$\begin{array}{l}\text { SA-modified } \mathrm{CaCO} 3 \\
\quad(70)\end{array}$} & 5.2 & uhsm + ext & 32 & -1 & - & - & 50 & - & [118] \\
\hline & 12.9 & & 41 & -3 & - & - & 150 & - & \\
\hline & 30.2 & & 65 & -9 & - & - & 350 & - & \\
\hline & 46.5 & & 70 & -15 & - & - & 220 & - & \\
\hline \multirow{3}{*}{$\begin{array}{l}\text { SEBS-g-MAH } \\
\text { modified } \mathrm{TiO}_{2}\end{array}$} & 1 & ext & 6 & 4 & - & - & - & - & [250] \\
\hline & 3 & & 10 & 7 & - & - & - & - & \\
\hline & 5 & & 16 & 6 & - & - & - & - & \\
\hline \multirow{3}{*}{$\begin{array}{l}\text { SEBS-g-MAH } \\
\text { modified ZnO }\end{array}$} & 1 & ext & 2 & 3 & - & - & - & - & [250] \\
\hline & 3 & & 8 & 2 & - & - & - & - & \\
\hline & 5 & & 9 & 4 & - & - & - & - & \\
\hline \multirow[t]{3}{*}{$\mathrm{ZnO}(30-40)$} & 5 & ext & - & - & - & - & - & 2 & [131] \\
\hline & 10 & & - & - & - & - & - & 1 & \\
\hline & 15 & & - & - & - & - & - & 1 & \\
\hline \multirow[t]{4}{*}{$\mathrm{ZnO}(10-30)$} & 1 & knd + minj & - & 29 & 27 & 15 & - & - & [129] \\
\hline & 3 & & - & 38 & 73 & 37 & - & - & \\
\hline & 5 & & - & 46 & 91 & 48 & - & - & \\
\hline & 15 & & - & 37 & 105 & 55 & - & - & [126] \\
\hline \multirow[t]{4}{*}{$\mathrm{C}_{3} \mathrm{~N}_{4}(100-300)$} & 0.1 & $\mathrm{mb}$ & - & - & - & - & - & 48 & \\
\hline & 0.5 & & - & - & - & - & - & 49 & \\
\hline & 1 & & - & - & - & - & - & 51 & \\
\hline & 2 & & - & - & - & - & - & 47 & \\
\hline \multirow{4}{*}{$\begin{array}{l}\text { GPTMS-modified } \\
\mathrm{Si}_{3} \mathrm{~N}_{4}(60-80)\end{array}$} & 0.1 & ext & - & -1 & - & - & 4 & - & [124] \\
\hline & 0.3 & & - & -2 & - & - & 11 & - & \\
\hline & 1 & & - & -4 & - & - & 22 & - & \\
\hline & 2 & & - & -10 & - & - & 33 & - & \\
\hline
\end{tabular}

$E$ : Young's modulus; $\sigma_{\mathrm{y}}$ : tensile strength at yield; $E_{\mathrm{f}}$ : flexural modulus; $\sigma_{\mathrm{fm}}:$ flexural strength; $E_{\text {charpy }}:$ impact strength; $E^{\prime}:$ storage modulus.

Key: $\mathrm{mb}=$ melt blending; isp = in situ polymerization; ext = extrusion; ult =ultrasonication; Knd = kneading; minj = micro injection molding; uhsm = ult rahigh speed mixing.

APTES: Aminopropyltriethoxysilane; GPTMS: Glycidyloxypropyltrimethoxysilane; HMDS: hexamethyldisilazane; MA: malonic acid; MAH: maleic anhydridre; PS: polystyrene; PVA: poly(vinyl alcohol); PBA: poly(butyl acrylate); POSS: polyhedral oligomeric silsesquioxane; SA: stearic acid; SEBS: styrene ethylene butylene styrene. 
Table 8

Comparison between the mechanical properties of nylon/IF-WS 2 and other nylon/inorganic nanoparticle composites.

\begin{tabular}{|c|c|c|c|c|c|c|c|c|c|}
\hline $\begin{array}{l}\text { Nanofiller (Particle } \\
\text { size/nm) }\end{array}$ & $\begin{array}{l}\text { Nanofiller content } \\
\text { (wt.\%) }\end{array}$ & $\begin{array}{l}\text { Processing } \\
\text { method }\end{array}$ & $\Delta E(\%)$ & $\Delta \sigma_{y}(\%)$ & $\Delta E_{\mathrm{f}}(\%)$ & $\Delta \sigma_{\mathrm{fm}}(\%)$ & $\Delta E_{\text {charpy }}(\%)$ & $\Delta E^{\prime}(\%)$ & Ref. \\
\hline IF-WS $2(40-180)$ & $\begin{array}{l}0.1 \\
0.5 \\
1 \\
2\end{array}$ & $\mathrm{mb}$ & $\begin{array}{l}- \\
- \\
- \\
-\end{array}$ & $\begin{array}{l}- \\
- \\
- \\
-\end{array}$ & $\begin{array}{l}- \\
- \\
- \\
-\end{array}$ & $\begin{array}{l}- \\
- \\
- \\
-\end{array}$ & $\begin{array}{l}- \\
- \\
- \\
-\end{array}$ & $\begin{array}{r}-2 \\
6 \\
48 \\
43\end{array}$ & [167] \\
\hline $\mathrm{C}_{60}$ & $\begin{array}{l}0.01 \\
0.02 \\
0.04 \\
0.06 \\
0.08\end{array}$ & isp & $\begin{array}{r}12 \\
10 \\
9 \\
8 \\
6\end{array}$ & $\begin{array}{r}14 \\
15 \\
13 \\
8 \\
8\end{array}$ & $\begin{array}{l}- \\
- \\
- \\
-\end{array}$ & $\begin{array}{l}- \\
- \\
- \\
- \\
-\end{array}$ & $\begin{array}{l}- \\
- \\
- \\
-\end{array}$ & $\begin{array}{l}- \\
- \\
- \\
- \\
-\end{array}$ & [265] \\
\hline$C_{60} / C_{70}$ & $\begin{array}{l}0.01 \\
0.02 \\
0.04 \\
0.06 \\
0.08\end{array}$ & isp & $\begin{array}{r}9 \\
11 \\
10 \\
9 \\
8\end{array}$ & $\begin{array}{l}20 \\
15 \\
11 \\
10 \\
10\end{array}$ & $\begin{array}{l}- \\
- \\
- \\
- \\
-\end{array}$ & $\begin{array}{l}- \\
- \\
- \\
- \\
-\end{array}$ & $\begin{array}{l}- \\
- \\
- \\
- \\
-\end{array}$ & $\begin{array}{l}- \\
- \\
- \\
- \\
-\end{array}$ & [265] \\
\hline $\mathrm{SiO}_{2}(50)$ & $\begin{array}{l}0.2 \\
0.5\end{array}$ & isp & $\begin{array}{r}7 \\
12\end{array}$ & $\begin{array}{l}10 \\
19\end{array}$ & $\begin{array}{l}- \\
-\end{array}$ & $\begin{array}{l}4 \\
7\end{array}$ & $\begin{array}{l}38 \\
17\end{array}$ & $\begin{array}{l}- \\
-\end{array}$ & [173] \\
\hline $\mathrm{SiO}_{2}(<50)$ & $\begin{array}{l}1 \\
2\end{array}$ & ext & $\begin{array}{l}27 \\
45\end{array}$ & $\begin{array}{l}18 \\
26\end{array}$ & $\begin{array}{l}- \\
-\end{array}$ & $\begin{array}{l}- \\
-\end{array}$ & $\begin{array}{l}- \\
-\end{array}$ & $\begin{array}{l}- \\
-\end{array}$ & [172] \\
\hline $\mathrm{SiO}_{2}(10-20)$ & $\begin{array}{c}1.8 \\
3.2 \\
7 \\
14\end{array}$ & ext & $\begin{array}{l}10 \\
18 \\
30 \\
44\end{array}$ & $\begin{array}{l}- \\
- \\
- \\
-\end{array}$ & $\begin{array}{l}- \\
- \\
- \\
-\end{array}$ & $\begin{array}{l}- \\
- \\
- \\
-\end{array}$ & $\begin{array}{l}- \\
- \\
- \\
-\end{array}$ & $\begin{array}{r}14 \\
24 \\
- \\
30\end{array}$ & [264] \\
\hline $\mathrm{SiO}_{2}(25-35)$ & $\begin{array}{l}1 \\
2 \\
3 \\
5\end{array}$ & isp & $\begin{array}{r}36 \\
44 \\
-33 \\
-42\end{array}$ & $\begin{array}{r}9 \\
11 \\
8 \\
6\end{array}$ & $\begin{array}{l}- \\
- \\
- \\
-\end{array}$ & $\begin{array}{l}- \\
- \\
- \\
-\end{array}$ & $\begin{array}{r}5 \\
16 \\
16 \\
-12\end{array}$ & $\begin{array}{l}- \\
- \\
- \\
-\end{array}$ & [149] \\
\hline $\mathrm{SiO}_{2}(10)$ & 4.3 & isp & - & 12 & - & - & -50 & 25 & [262] \\
\hline $\mathrm{SiO}_{2}(<100)$ & $\begin{array}{r}5 \\
10\end{array}$ & isp & $\begin{array}{l}10 \\
20\end{array}$ & $\begin{array}{l}-1 \\
-2\end{array}$ & $\begin{array}{l}- \\
-\end{array}$ & - & $\begin{array}{r}-6 \\
-12\end{array}$ & $\begin{array}{l}- \\
-\end{array}$ & [85] \\
\hline $\begin{array}{l}\text { APTES-modified } \\
\mathrm{SiO}_{2}(10)\end{array}$ & 4.3 & isp & - & 6 & - & - & 26 & 0 & [262] \\
\hline $\begin{array}{l}\text { CA-modified } \mathrm{SiO}_{2} \\
(50)\end{array}$ & $\begin{array}{l}0.2 \\
0.5\end{array}$ & isp & $\begin{array}{l}27 \\
26\end{array}$ & $\begin{array}{l}31 \\
31\end{array}$ & $\begin{array}{l}- \\
-\end{array}$ & $\begin{array}{l}33 \\
21\end{array}$ & $\begin{array}{l}93 \\
63\end{array}$ & $\begin{array}{l}- \\
-\end{array}$ & [173] \\
\hline $\begin{array}{l}\mathrm{NH}_{2}-\mathrm{A} \text {-modified } \\
\mathrm{SiO}_{2}(<100)\end{array}$ & $\begin{array}{r}5 \\
\\
10 \\
15\end{array}$ & isp & $\begin{array}{l}14 \\
22 \\
27\end{array}$ & $\begin{array}{r}12 \\
4 \\
0\end{array}$ & $\begin{array}{l}- \\
- \\
-\end{array}$ & $\begin{array}{l}- \\
- \\
-\end{array}$ & $\begin{array}{r}74 \\
38 \\
9\end{array}$ & $\begin{array}{l}- \\
- \\
-\end{array}$ & [85] \\
\hline $\mathrm{CaCO}_{3}(11)$ & $\begin{array}{l}1 \\
2 \\
3 \\
4\end{array}$ & ext & $\begin{array}{l}320 \\
420 \\
460 \\
570\end{array}$ & $\begin{array}{l}23 \\
31 \\
35 \\
38\end{array}$ & $\begin{array}{l}- \\
- \\
- \\
-\end{array}$ & $\begin{array}{l}- \\
- \\
- \\
-\end{array}$ & $\begin{array}{l}- \\
- \\
- \\
-\end{array}$ & $\begin{array}{l}- \\
- \\
- \\
-\end{array}$ & [176] \\
\hline $\mathrm{CaCO}_{3}(50)$ & $\begin{array}{r}5 \\
10 \\
20\end{array}$ & $\mathrm{mb}$ & $\begin{array}{r}-13 \\
-2 \\
-9\end{array}$ & $\begin{array}{l}-25 \\
-33 \\
-29\end{array}$ & $\begin{array}{l}- \\
- \\
-\end{array}$ & $\begin{array}{l}- \\
- \\
-\end{array}$ & $\begin{array}{l}-27 \\
-40 \\
-33\end{array}$ & $\begin{array}{l}- \\
- \\
-\end{array}$ & [261] \\
\hline $\begin{array}{l}\text { SA-modified } \mathrm{CaCO}_{3} \\
\quad(50)\end{array}$ & $\begin{array}{r}3 \\
5 \\
10 \\
20\end{array}$ & $\mathrm{mb}$ & $\begin{array}{r}-6 \\
9 \\
34 \\
36\end{array}$ & $\begin{array}{r}-1 \\
1 \\
-23 \\
-34\end{array}$ & $\begin{array}{l}- \\
- \\
- \\
-\end{array}$ & $\begin{array}{l}- \\
- \\
- \\
-\end{array}$ & $\begin{array}{r}-6 \\
33 \\
-50 \\
-65\end{array}$ & $\begin{array}{r}0 \\
3 \\
6 \\
16\end{array}$ & [261] \\
\hline $\begin{array}{l}\mathrm{NH}_{2}-\mathrm{A}-\text { modified } \\
\mathrm{CaCO}_{3}(50)\end{array}$ & $\begin{array}{r}5 \\
20\end{array}$ & $\mathrm{mb}$ & $\begin{array}{l}14 \\
40\end{array}$ & $\begin{array}{l}-16 \\
-23\end{array}$ & $\begin{array}{l}- \\
-\end{array}$ & $\begin{array}{l}- \\
-\end{array}$ & $\begin{array}{l}-20 \\
-52\end{array}$ & $\begin{array}{l}- \\
-\end{array}$ & [261] \\
\hline $\begin{array}{l}\text { APTES-modified } \\
\mathrm{Al}_{2} \mathrm{O}_{3}(80)\end{array}$ & $\begin{array}{l}1 \\
2 \\
3 \\
4\end{array}$ & isp & $\begin{array}{l}- \\
- \\
- \\
-\end{array}$ & $\begin{array}{l}13 \\
38 \\
53 \\
46\end{array}$ & $\begin{array}{l}- \\
- \\
- \\
-\end{array}$ & $\begin{array}{l}- \\
- \\
- \\
-\end{array}$ & $\begin{array}{l}- \\
- \\
- \\
-\end{array}$ & $\begin{array}{l}12 \\
41 \\
51 \\
32\end{array}$ & [263] \\
\hline
\end{tabular}


Table 8 (Continued)

\begin{tabular}{|c|c|c|c|c|c|c|c|c|c|}
\hline $\begin{array}{l}\text { Nanofiller (Particle } \\
\text { size/nm) }\end{array}$ & $\begin{array}{l}\text { Nanofiller content } \\
\text { (wt.\%) }\end{array}$ & $\begin{array}{l}\text { Processing } \\
\text { method }\end{array}$ & $\Delta E(\%)$ & $\Delta \sigma_{y}(\%)$ & $\Delta E_{\mathrm{f}}(\%)$ & $\Delta \sigma_{\mathrm{fm}}(\%)$ & $\Delta E_{\text {charpy }}(\%)$ & $\Delta E^{\prime}(\%)$ & Ref. \\
\hline \multirow[t]{4}{*}{$\mathrm{TiO}_{2}$} & 2 & isp & 4 & 3 & 5 & 5 & -7 & - & [174] \\
\hline & 4 & & 12 & 5 & 12 & 11 & -11 & - & \\
\hline & 6 & & 28 & 7 & 17 & 14 & -34 & - & \\
\hline & 8 & & 32 & 9 & 26 & 17 & -35 & - & \\
\hline \multirow{4}{*}{$\begin{array}{l}\text { APTES-modified } \\
\mathrm{TiO}_{2}\end{array}$} & 2 & isp & 7 & 5 & 12 & 5 & -6 & - & [174] \\
\hline & 4 & & 18 & 8 & 20 & 13 & -10 & - & \\
\hline & 6 & & 34 & 11 & 29 & 18 & -24 & - & \\
\hline & 8 & & 37 & 14 & 38 & 25 & -32 & - & \\
\hline $\mathrm{Si}_{3} \mathrm{~N}_{4}$ & 1 & $\mathrm{mb}$ & 276 & 179 & - & - & - & - & [217] \\
\hline
\end{tabular}

$E$ : Young's modulus; $\sigma_{\mathrm{y}}$ : tensile strength at yield; $E_{\mathrm{f}}$ : flexural modulus; $\sigma_{\mathrm{fm}}$ : flexural strength; $E_{\text {charpy }}$ impact strength; $E^{\prime}$ : storage modulus.

Key: $\mathrm{mb}=$ melt blending; isp = in situ polymerization; ext = extrusion.

APTES: Aminopropyltriethoxysilane; $\mathrm{NH}_{2}-\mathrm{A}$ : amino acid; $\mathrm{SA}$ : stearic acid.

hence satisfactory mechanical properties. Notwithstanding, a very efficient dispersion of IF-WS 2 in iPP by simple melt-blending without the aid of surfactants or modifiers was reported by Naffakh et al. $[93,244]$. The addition of $1 \mathrm{wt} . \% \mathrm{IF}-\mathrm{WS}_{2}$ led to significant increases in the Young's and flexural modulus, tensile and flexural strength, by $\sim 39$, 29, 36 and 17\%, respectively, Table 7. As shown in Section 4.3 , these nanoparticles are efficient $\alpha$-NAs, promoting the formation of $\alpha$-spherulites with a radial lamellar architecture that improves the stiffness and strength of iPP [245]. In contrast, the impact strength of iPP/IF-WS 2 (1 wt.\%) was $\sim 12 \%$ lower than that of the neat matrix due to a significant reduction in the elongation at break because the interlocking effect of the radial lamellae by the tangential crystallites hinders plastic deformation [246]. Thus, IF$\mathrm{WS}_{2}$ nanoparticles are effective for enhancing the stiffness and strength of iPP at the expense of toughness. However, it should be highlighted that the improvements in the tensile and flexural properties attained by incorporating $1 \mathrm{wt} . \%$ IF-WS 2 are considerably larger than those found in composites reinforced with the equivalent concentration of other modified or unmodified nanofillers such as $\mathrm{SiO}_{2}$ [94,191], $\mathrm{Al}_{2} \mathrm{O}_{3}$ [247,248], $\mathrm{CaCO}_{3}$ [249], $\mathrm{TiO}_{2}$ [250] or $\mathrm{Si}_{3} \mathrm{~N}_{4}$ [124], Table 7 . Only the addition of $1 \mathrm{wt} . \% \mathrm{ZnO}$ via kneading and microinjection molding [129] led to quantitatively similar increments in the flexural properties of $\mathrm{PP}$, due to the nucleating effect of these nanoparticles that were well dispersed within the matrix. On the other hand,

Table 9

Comparison between the mechanical properties of PPS/IF-WS 2 and other PPS/inorganic nanoparticle composites.

\begin{tabular}{|c|c|c|c|c|c|c|c|c|c|}
\hline $\begin{array}{l}\text { Nanofiller (Particle } \\
\text { size/nm) }\end{array}$ & $\begin{array}{l}\text { Nanofiller content } \\
\text { (wt.\%) }\end{array}$ & Processing method & $\Delta E(\%)$ & $\Delta \sigma_{\mathrm{y}}(\%)$ & $\Delta E_{\mathrm{f}}(\%)$ & $\Delta \sigma_{\mathrm{fm}}(\%)$ & $\Delta E_{\text {charpy }}(\%)$ & $\Delta E^{\prime}(\%)$ & Ref. \\
\hline \multirow{4}{*}{ IF-WS $2(40-180)$} & 0.5 & \multirow[t]{4}{*}{$\mathrm{mb}$} & - & - & - & - & - & 74 & \multirow[t]{4}{*}[168,266]{} \\
\hline & 1 & & - & - & - & - & - & 70 & \\
\hline & 2 & & 18 & 14 & - & - & 8 & 45 & \\
\hline & 8 & & - & - & - & - & - & 75 & \\
\hline Epoxy-g-SiO 2 (5-15) & 3 & $b m l+m b$ & - & - & - & - & 73 & - & [164] \\
\hline \multirow{4}{*}{$\mathrm{Al}_{2} \mathrm{O}_{3}$} & 1.5 & \multirow[t]{4}{*}{$\mathrm{mb}+\mathrm{cm}$} & - & - & - & -25 & - & - & \multirow[t]{4}{*}{ [269] } \\
\hline & 2.9 & & - & - & - & -42 & - & - & \\
\hline & 4.4 & & - & - & - & -21 & - & - & \\
\hline & 7.3 & & - & - & - & -10 & - & - & \\
\hline \multirow[t]{4}{*}{$\mathrm{CaCO}_{3}(40-60)$} & 2 & \multirow[t]{4}{*}{ ext } & - & 4 & - & - & 182 & - & \multirow[t]{4}{*}{ [268] } \\
\hline & 3 & & - & 7 & - & - & 200 & - & \\
\hline & 5 & & - & 3 & - & - & 125 & - & \\
\hline & 10 & & - & -1 & - & - & -2 & - & \\
\hline \multirow[t]{4}{*}{$\mathrm{CuO}(30-50)$} & 4.6 & \multirow[t]{4}{*}{$\mathrm{mb}+\mathrm{cm}$} & - & - & - & -23 & - & - & \multirow[t]{4}{*}[270]{} \\
\hline & 8.8 & & - & - & - & -26 & - & - & \\
\hline & 12.8 & & - & - & - & -15 & - & - & \\
\hline & 34.5 & & - & - & - & -8 & - & - & \\
\hline \multirow[t]{4}{*}{$\mathrm{TiO}_{2}(30-50)$} & 3.1 & \multirow[t]{2}{*}{$m b+c m$} & - & - & - & -17 & - & - & \multirow[t]{4}{*}[270]{} \\
\hline & 6 & & - & - & - & -11 & - & - & \\
\hline & 8.8 & $\mathrm{mb}+\mathrm{cm}$ & - & - & - & -8 & - & - & \\
\hline & 14.2 & & - & - & - & -3 & - & - & \\
\hline \multirow[t]{3}{*}{$\mathrm{ZnO}(30-50)$} & 4 & & - & - & - & -15 & - & - & \multirow[t]{3}{*}{ [270] } \\
\hline & 7.8 & & - & - & - & -18 & - & - & \\
\hline & 17.9 & & - & - & - & -5 & - & - & \\
\hline
\end{tabular}

$E$ : Young's modulus; $\sigma_{\mathrm{y}}$ : tensile strength at yield; $E_{\mathrm{f}}$ : flexural modulus; $\sigma_{\mathrm{fm}}$ : flexural strength; $E_{\text {charpy }}$ : impact strength; $E^{\prime}$ : storage modulus. Key: $\mathrm{mb}=$ melt blending; ext $=$ extrusion; bml=ball milling; $\mathrm{cm}=$ compression molding. 
Table 10

Comparison between the mechanical properties of PEEK/IF-WS ${ }_{2}$ and other PEEK/inorganic nanoparticle composites.

\begin{tabular}{|c|c|c|c|c|c|c|c|c|c|}
\hline $\begin{array}{l}\text { Nanofiller (Particle } \\
\text { size/nm) }\end{array}$ & $\begin{array}{l}\text { Nanofiller content } \\
\text { (wt.\%) }\end{array}$ & $\begin{array}{l}\text { Processing } \\
\text { method }\end{array}$ & $\Delta E(\%)$ & $\Delta \sigma_{y}(\%)$ & $\Delta E_{\mathrm{f}}(\%)$ & $\Delta \sigma_{\mathrm{fm}}(\%)$ & $\Delta E_{\text {charpy }}(\%)$ & $\Delta E^{\prime}(\%)$ & Ref. \\
\hline IF-WS $(40-180)$ & $\begin{array}{l}0.1 \\
0.5 \\
1 \\
2\end{array}$ & $\mathrm{Bml}+\mathrm{mb}$ & $\begin{array}{l}10 \\
20 \\
29 \\
37\end{array}$ & $\begin{array}{r}2 \\
4 \\
8 \\
14\end{array}$ & $\begin{array}{l}- \\
- \\
- \\
-\end{array}$ & $\begin{array}{l}- \\
- \\
- \\
-\end{array}$ & $\begin{array}{r}20 \\
7 \\
-12 \\
-25\end{array}$ & $\begin{array}{r}8 \\
17 \\
28 \\
33\end{array}$ & [57] \\
\hline $\mathrm{IF}-\mathrm{WS}_{2}(80-220)^{\mathrm{a}}$ & $\begin{array}{c}1 \\
2.5 \\
5 \\
10 \\
20\end{array}$ & Aed & $\begin{array}{r}13 \\
7 \\
17 \\
52 \\
61\end{array}$ & $\begin{array}{l}- \\
- \\
- \\
- \\
-\end{array}$ & $\begin{array}{l}- \\
- \\
- \\
- \\
-\end{array}$ & $\begin{array}{l}- \\
- \\
- \\
- \\
-\end{array}$ & $\begin{array}{l}- \\
- \\
- \\
-\end{array}$ & $\begin{array}{l}- \\
- \\
- \\
- \\
-\end{array}$ & [58] \\
\hline $\mathrm{SiO}_{2}(13)$ & $\begin{array}{l}1 \\
2.1 \\
4.2 \\
8.4\end{array}$ & $\mathrm{Bml}+\mathrm{cm}$ & $\begin{array}{l}12 \\
14 \\
19 \\
15\end{array}$ & $\begin{array}{r}2 \\
-1 \\
1 \\
0\end{array}$ & $\begin{array}{l}- \\
- \\
- \\
-\end{array}$ & $\begin{array}{l}- \\
- \\
- \\
-\end{array}$ & $\begin{array}{l}- \\
- \\
- \\
-\end{array}$ & $\begin{array}{l}- \\
- \\
- \\
-\end{array}$ & [218] \\
\hline $\mathrm{SiO}_{2}(30)$ & $\begin{array}{c}2.5 \\
5 \\
7.5 \\
10\end{array}$ & $\mathrm{Ult}+\mathrm{cm}$ & $\begin{array}{r}8 \\
15 \\
26 \\
36\end{array}$ & $\begin{array}{r}6 \\
18 \\
2 \\
0\end{array}$ & $\begin{array}{l}- \\
- \\
- \\
-\end{array}$ & $\begin{array}{l}- \\
- \\
- \\
-\end{array}$ & $\begin{array}{l}- \\
- \\
- \\
-\end{array}$ & $\begin{array}{l}- \\
- \\
- \\
-\end{array}$ & [152] \\
\hline $\mathrm{SiO}_{2}(30)$ & $\begin{array}{c}2.5 \\
5 \\
7.5 \\
10\end{array}$ & $\mathrm{Ult}+\mathrm{cm}$ & $\begin{array}{r}3 \\
5 \\
10 \\
13\end{array}$ & $\begin{array}{r}5 \\
6 \\
-7 \\
-7\end{array}$ & $\begin{array}{l}- \\
- \\
- \\
-\end{array}$ & $\begin{array}{l}- \\
- \\
- \\
-\end{array}$ & $\begin{array}{l}- \\
- \\
- \\
-\end{array}$ & $\begin{array}{l}20 \\
29 \\
31 \\
36\end{array}$ & [159] \\
\hline $\begin{array}{l}\text { SA-modified } \mathrm{SiO}_{2} \\
\quad(30)\end{array}$ & $\begin{array}{c}2.5 \\
\\
5 \\
7.5 \\
10\end{array}$ & $\mathrm{Ult}+\mathrm{cm}$ & $\begin{array}{c}8 \\
10 \\
15 \\
18\end{array}$ & $\begin{array}{r}3 \\
7 \\
1 \\
-6\end{array}$ & $\begin{array}{l}- \\
- \\
- \\
-\end{array}$ & $\begin{array}{l}- \\
- \\
- \\
-\end{array}$ & $\begin{array}{l}- \\
- \\
- \\
-\end{array}$ & $\begin{array}{l}22 \\
31 \\
32 \\
40\end{array}$ & [159] \\
\hline $\mathrm{Al}_{2} \mathrm{O}_{3}(30)$ & $\begin{array}{l}2.5 \\
5 \\
7.5 \\
10\end{array}$ & $\mathrm{Ult}+\mathrm{cm}$ & $\begin{array}{r}5 \\
13 \\
18 \\
31\end{array}$ & $\begin{array}{r}9 \\
18 \\
21 \\
6\end{array}$ & $\begin{array}{l}- \\
- \\
- \\
-\end{array}$ & $\begin{array}{l}- \\
- \\
- \\
-\end{array}$ & $\begin{array}{l}- \\
- \\
- \\
-\end{array}$ & $\begin{array}{l}- \\
- \\
- \\
-\end{array}$ & [152] \\
\hline $\begin{array}{l}\mathrm{Al}_{2} \mathrm{O}_{3}(15) \\
\mathrm{SA} \text {-modified } \mathrm{Al}_{2} \mathrm{O}_{3} \\
\quad(15)\end{array}$ & $\begin{array}{l}5 \\
5\end{array}$ & $\begin{array}{l}\mathrm{md}+\mathrm{cm} \\
\mathrm{md}+\mathrm{cm}\end{array}$ & $\begin{array}{l}- \\
-\end{array}$ & $\begin{array}{l}7 \\
7\end{array}$ & $\begin{array}{l}- \\
-\end{array}$ & $\begin{array}{l}30 \\
16\end{array}$ & $\begin{array}{l}260 \\
620\end{array}$ & - & $\begin{array}{l}{[275]} \\
{[275]}\end{array}$ \\
\hline $\begin{array}{l}\text { Titanate-modified } \\
\mathrm{Al}_{2} \mathrm{O}_{3}(15)\end{array}$ & $\begin{array}{r}5 \\
10\end{array}$ & $\mathrm{md}+\mathrm{cm}$ & $\begin{array}{l}3 \\
3\end{array}$ & $\begin{array}{r}7 \\
-2\end{array}$ & $\begin{array}{l}- \\
-\end{array}$ & $\begin{array}{l}20 \\
29\end{array}$ & $\begin{array}{l}660 \\
275\end{array}$ & - & [275] \\
\hline $\mathrm{Al}_{2} \mathrm{O}_{3}(3-15)$ & $\begin{array}{l}10 \\
20 \\
30 \\
50 \\
60\end{array}$ & $\mathrm{mst}+\mathrm{hc}$ & $\begin{array}{l}- \\
- \\
- \\
- \\
-\end{array}$ & $\begin{array}{l}- \\
- \\
- \\
- \\
-\end{array}$ & $\begin{array}{l}- \\
- \\
- \\
- \\
-\end{array}$ & $\begin{array}{l}- \\
- \\
- \\
- \\
-\end{array}$ & $\begin{array}{l}- \\
- \\
- \\
- \\
-\end{array}$ & $\begin{array}{l}20 \\
25 \\
50 \\
72 \\
78\end{array}$ & [276] \\
\hline $\mathrm{Al}_{2} \mathrm{O}_{3}(20)$ & $\begin{array}{l}1 \\
2 \\
3\end{array}$ & $\mathrm{mb}$ & $\begin{array}{l}20 \\
28 \\
40\end{array}$ & $\begin{array}{r}5 \\
8 \\
10\end{array}$ & $\begin{array}{r}16 \\
170 \\
100\end{array}$ & $\begin{array}{r}6 \\
13 \\
15\end{array}$ & $\begin{array}{l}53 \\
64 \\
90\end{array}$ & $\begin{array}{l}- \\
- \\
-\end{array}$ & [271] \\
\hline $\operatorname{AIN}(2-10)$ & $\begin{array}{l}10 \\
20 \\
50 \\
60 \\
70\end{array}$ & mst + hc & $\begin{array}{l}- \\
- \\
- \\
- \\
-\end{array}$ & $\begin{array}{l}- \\
- \\
- \\
- \\
-\end{array}$ & $\begin{array}{l}- \\
- \\
- \\
- \\
-\end{array}$ & $\begin{array}{l}- \\
- \\
- \\
- \\
-\end{array}$ & $\begin{array}{l}- \\
- \\
- \\
-\end{array}$ & $\begin{array}{r}18 \\
40 \\
150 \\
300 \\
350\end{array}$ & [276] \\
\hline $\mathrm{CaCO}_{3}(80)$ & $\begin{array}{r}5 \\
10 \\
15 \\
20 \\
25 \\
30\end{array}$ & ext & $\begin{array}{r}6 \\
12 \\
18 \\
25 \\
25 \\
41\end{array}$ & $\begin{array}{r}-5 \\
-4 \\
-4 \\
-5 \\
-6 \\
4\end{array}$ & $\begin{array}{r}2 \\
11 \\
17 \\
16 \\
32 \\
35\end{array}$ & $\begin{array}{r}4 \\
9 \\
13 \\
13 \\
33 \\
45\end{array}$ & $\begin{array}{l}-20 \\
-47 \\
-49 \\
-53 \\
-58 \\
-53\end{array}$ & $\begin{array}{l}- \\
- \\
- \\
- \\
- \\
-\end{array}$ & [274] \\
\hline $\begin{array}{l}\text { SPEEK-modified } \\
\mathrm{CaCO}_{3}(80)\end{array}$ & $\begin{array}{l}5 \\
\\
10 \\
15 \\
20\end{array}$ & ext & $\begin{array}{l}13 \\
21 \\
22 \\
22\end{array}$ & $\begin{array}{r}-1 \\
1 \\
-1 \\
5\end{array}$ & $\begin{array}{r}2 \\
7 \\
7 \\
17 \\
18\end{array}$ & $\begin{array}{l}3 \\
3 \\
4 \\
7\end{array}$ & $\begin{array}{l}-28 \\
-35 \\
-44 \\
-46\end{array}$ & $\begin{array}{l}- \\
- \\
- \\
-\end{array}$ & [274] \\
\hline
\end{tabular}


Table 10 (Contimued)

\begin{tabular}{|c|c|c|c|c|c|c|c|c|c|}
\hline $\begin{array}{l}\text { Nanofiller (Particle } \\
\text { size/nm) }\end{array}$ & $\begin{array}{l}\text { Nanofiller content } \\
\text { (wt.\%) }\end{array}$ & $\begin{array}{l}\text { Processing } \\
\text { method }\end{array}$ & $\Delta E(\%)$ & $\Delta \sigma_{y}(\%)$ & $\Delta E_{\mathrm{f}}(\%)$ & $\Delta \sigma_{\mathrm{fm}}(\%)$ & $\Delta E_{\text {charpy }}(\%)$ & $\Delta E^{\prime}(\%)$ & Ref. \\
\hline & 25 & & 27 & 5 & 26 & 15 & -62 & - & \\
\hline & 30 & & 25 & 4 & 25 & 23 & -72 & - & \\
\hline \multirow[t]{6}{*}{$\mathrm{TiO}_{2}(15)$} & 2.6 & ext & 4 & 18 & - & - & 70 & - & {$[272,273]$} \\
\hline & 5.9 & & 7 & 10 & - & - & 70 & - & \\
\hline & 9 & & 11 & 25 & - & - & 148 & 8 & \\
\hline & 18.2 & & 22 & 26 & - & - & 80 & - & \\
\hline & 25.5 & & 36 & 27 & - & - & -15 & 40 & \\
\hline & 28.8 & & 39 & 34 & - & - & 2 & - & \\
\hline \multirow[t]{5}{*}{$\mathrm{ZnS}(300)$} & 4.4 & ext & 14 & -8 & - & - & - & - & [272] \\
\hline & 8.4 & & 11 & 7 & - & - & - & - & \\
\hline & 13.4 & & 18 & -4 & - & - & - & - & \\
\hline & 18.5 & & 25 & -16 & - & - & - & - & \\
\hline & 25 & & 31 & 0 & - & - & - & - & \\
\hline
\end{tabular}

$E$ : Young's modulus; $\sigma_{\mathrm{y}}$ : tensile strength at yield; $E_{\mathrm{f}}$ : flexural modulus; $\sigma_{\mathrm{fm}}$ : flexural strength; $E_{\text {charpy }}$ : impact strength; $E^{\prime}$ : storage modulus. Key: $\mathrm{mb}=$ melt blending; ext = extrusion; $\mathrm{bml}=$ ball milling; aed = aerosol assisted deposition; md = mechanical dispersion; $m$ st = magnetic stirring; hc = hotcompression.

SA: stearic acid; SPEEK: sulfonated poly(ether ether ketone).

a Nanocomposite coating.

the incorporation of $1 \mathrm{wt} \% \mathrm{Al}_{2} \mathrm{O}_{3}$ [247] provoked a moderate decrease in the tensile properties and hardly modified the flexural behavior of the polymer. Analogously, a slight drop in the tensile strength was found by the addition of the same amount of $\mathrm{SiO}_{2}$ [191], ascribed to the filler agglomerates that acted as failure initiation sites facilitating crack propagation. Moreover, the surface of these nanoparticles is polar whereas PP is non-polar, hence the interface becomes incompatible. After surface treatment of the $\mathrm{SiO}_{2}$ with a silane coupling agent [191], or addition of a compatibilizer such as PP-g-MAH or PP-methyl-POSS [114], the nanoparticle-matrix interface improved and agglomeration was reduced, resulting in an increase in the Young's modulus and tensile strength compared to the composites with untreated nanoparticles, although the values achieved remained considerably lower than for $\mathrm{iPP} / \mathrm{IF}-\mathrm{WS}_{2}$. In the case of $\mathrm{iPP} / \mathrm{Si}_{3} \mathrm{~N}_{4}$ (1 wt.\%) nanocomposite [124], a small reduction in the tensile strength was also observed, ascribed to a decrease in the crystallinity of the matrix. The addition of $1 \mathrm{wt} \%$ untreated $\mathrm{CaCO}_{3}$ [249], a weak $\alpha$ NA, hardly improved the tensile strength of iPP, while the incorporation of malonic acid (MA) as a surface modifier, which favors the formation of the $\beta$-phase, resulted in a moderate increase in this parameter, ascribed to a $\beta$ to $\alpha$ transition occurring during the tensile process that led to a strain hardening. However, the flexural modulus of iPP/MA treated $\mathrm{CaCO}_{3}$ was lower than that of untreated nanocomposites. Both types of $\mathrm{CaCO}_{3}$ reinforced nanocomposites displayed inferior properties than iPP/IF-WS 2 at $1 \mathrm{wt} . \%$ loading. Only the addition of $\geq 7 \mathrm{wt} . \% \mathrm{CaCO}_{3}$ resulted in similar increments in the Young's modulus [251]. The incorporation of $1 \mathrm{wt} \% \mathrm{TiO}_{2}$ [250] coated with SEBS-g-MAH also resulted in smaller increases in the tensile properties. Despite the fact that the compatibilizer led to fine nanofiller dispersion and a strong $\mathrm{TiO}_{2}$-iPP interface, its elastomeric nature impaired property enhancement.

With regard to the impact strength, most of the nanofillers generated improvements, in contrast to the behavior found for the IF-WS 2 nanoparticles, Table 7 . The incorporation of $1 \mathrm{wt} . \% \mathrm{Al}_{2} \mathrm{O}_{3}$ [248], fumed $\mathrm{SiO}_{2}$ [94], silane treated $\mathrm{SiO}_{2}$ [191] or $\mathrm{Si}_{3} \mathrm{~N}_{4}$ [124], and untreated or MAtreated $\mathrm{CaCO}_{3}$ [249] improved the toughness of PP by $\sim 9,20,25,22,27$ and $129 \%$, respectively. The extraordinary increase in the latter composite was ascribed to a very homogenous nanofiller dispersion combined with the formation of small spherulites with high $\beta$-phase content that positively contribute to the toughness [252]. Strong enhancements in the toughness of iPP were also achieved by adding $4.5 \mathrm{wt} \% \mathrm{SiO}_{2}$ [253], and particularly 2.5 wt.\% PBA-g-SiO 2 [112], 7.6 wt.\% PS-g-SiO 2 or PVA-g$\mathrm{SiO}_{2}[110,254]$, ascribed to efficiently dispersed grafted nanoparticles with strong interfacial interactions with the matrix that favor the pinning of cracks. Furthermore, Wang et al. [118] found a 350\% impact strength increase upon addition of $\sim 30 \mathrm{wt} . \%$ SA-treated $\mathrm{CaCO}_{3}$ nanoparticles, and Cioni et al. [255] reported an unprecedented improvement of $\sim 550 \%$ with $\sim 24$ wt.\% SA-coated $\mathrm{CaCO}_{3}$, attributed to the formation of a continuous monolayer of hydrophobic alkyl chains on the nanoparticles that promotes a more uniform filler dispersion and matrix-particle debonding, allowing higher energy absorption during fracture.

DMA measurements are typically performed to monitor changes in the stiffness of the nanocomposites as a function of temperature and are also sensitive to the relaxation processes of the matrix, providing some information on nanoparticle-matrix interactions. For iPP/IF-WS 2 nanocomposites [93], the storage modulus, indicative of the elastic energy stored in the material, decreased progressively with increasing temperature and a more pronounced fall was encountered in the range $-10-30^{\circ} \mathrm{C}$, associated with the glass transition. At room temperature the modulus increased from $1500 \mathrm{MPa}$ for neat iPP to 1600,1800 and 2000 for nanocomposites incorporating $0.1,4$ and $8 \mathrm{wt} . \% \mathrm{IF}_{-} \mathrm{WS}_{2}$, respectively, explained in terms of the nucleating effect of these nanoparticles resulting in higher matrix crystallinity.

In general it can be noted that IF-WS 2 is more effective for improving the storage modulus of iPP than other nanofillers. The addition of $5-15 \mathrm{wt} \% \mathrm{CaCO}_{3}$ [117] or $\mathrm{ZnO}$ [131] hardly modified the room temperature modulus of 
the matrix, and the enhancements observed with 0.1 and 4 wt.\% IF-WS 2 are similar to those reported for iPP/SAmodified $\mathrm{CaCO}_{3}$ at $20 \mathrm{wt} . \%$ [199] and $\mathrm{iPP} / \mathrm{SiO}_{2}$ at $5.3 \mathrm{wt} . \%$ [256], respectively. Larger improvements of $\sim 48 \%$ were found for composites containing very small amounts, i.e. $0.1 \mathrm{wt} . \%$, of $\mathrm{C}_{3} \mathrm{~N}_{4}$ nanoparticles [126]. These are also $\alpha-$ NAs, and the improvements are explained by the nucleating effect combined with a very efficient dispersion within the matrix that strongly restricts free movement of the matrix macromolecular chains.

The damping or loss factor, $\tan \delta$ is another important parameter obtained from DMA measurements and is related to the impact resistance of the material. Two relaxation peaks were found in the $\tan \delta$ curves of $\mathrm{iPP} / \mathrm{IF}$ $\mathrm{WS}_{2}$ nanocomposites [93]; a lower temperature maximum, the $\beta$-relaxation, associated with the glass transition of the matrix, and a higher temperature maximum, the $\alpha$ transition, ascribed to the relaxation of restricted iPP amorphous chains in the crystalline phase (defects) [257]. The peak position of the $\beta$-transition temperature was only slightly affected by the nanofiller, however, the width of the peak increased significantly, by up to $30 \%$ for $8 w t . \%$ IF-WS ${ }_{2}$ contents. This broadening could also be attributed to a nucleation effect resulting in a more inhomogeneous amorphous phase in the composites compared to the neat matrix. Although the effect of $\mathrm{C}_{3} \mathrm{~N}_{4}$ [126], $\mathrm{CaCO}_{3}$ [117] and $\mathrm{ZnO}$ [131] on the $T_{g}$ of the matrix was negligible, restrictions on polymer chain mobility were reflected in a reduction in peak heights, hence lower damping characteristics.

\subsubsection{Nylon-6}

The mechanical properties of nylon- 6 are strongly conditioned by the morphology of the spherulites (size, shape, distribution, etc.) and the crystal phases. The pseudohexagonal $\gamma$-phase has twisted molecular chains and few inter-chain interactions and is less rigid than the monoclinic $\alpha$-phase, where the chains are packed in a stable antiparallel arrangement of hydrogen bonds [258]. The incorporation of small amounts of inorganic nanofillers usually alters the morphology of nylon, and is an effective way to improving mechanical performance, Table 8.

Very scarce information related to the mechanical properties of PA-6/IF-WS 2 composites has been published, and is limited exclusively to DMA measurements, Table 8 [167] where a remarkable increment in the matrix modulus of $\sim 48 \%$ at room temperature was observed on the incorporation of $1 \mathrm{wt} . \%$ IF-WS 2 , which was ascribed to a nucleating effect that promotes the formation of small imperfect crystals that increase the rigidity of crystalline PA-6. Smaller crystallites have more surface area and are expected to restrain the chain motion more efficiently than larger crystallites, leading to an increase in the relaxation temperatures. However, the $\alpha, \beta$ and $\gamma$ transitions occurring at $\sim 50,-60$ and $-125^{\circ} \mathrm{C}[259,260]$, related to the rupture of hydrogen bonds between chains in the amorphous region, the motion of chain segments including the amide groups and the crank-shaft motion mechanism of methylene sequences, respectively, were hardly influenced by the nanoparticles. A reduction in the width of the $\alpha-$ relaxation corresponding to the $T_{g}$ the nanocomposite with
1 wt.\% IF-WS 2 was observed compared to that of neat PA-6. Faster crystallization, due to the aforementioned nucleating effect, results in a more homogenous amorphous phase. However, no noticeable change in the peak height was observed. It should be noted that the improvements in the room temperature storage modulus found for PA$6 / I F-W_{2}$ nanocomposites are more significant than those reported for composites with other types of nanofillers. The incorporation of 10-20 wt.\% SA-modified $\mathrm{CaCO}_{3}$ [261], $4.3 \mathrm{wt} . \%$ untreated or silane-treated $\mathrm{SiO}_{2}$ [262] resulted in smaller increments in this parameter than the addition of $1-2 \mathrm{wt} . \%$ IF-WS 2 . Only the nanocomposite with $2 \mathrm{wt} . \%$ silane-modified $\mathrm{Al}_{2} \mathrm{O}_{3}$ [263] exhibited similar enhancement to that found by adding the same amount of IF-WS 2 . On the other hand, the incorporation of $\mathrm{SiO}_{2}$ at $1.8-14$ wt.\% [264] did not change significantly the $T_{g}$ of the matrix, similarly to the behavior found for the IF-WS 2 nanoparticles, albeit led to a decrease in $\tan \delta_{\max }$ value, indicating improved load bearing properties of the nanocomposites compared to neat PA-6. A diminution in the height of $\tan \delta$ maximum was also observed for composites filled with SA or $\mathrm{NH}_{2}-\mathrm{A}$-modified $\mathrm{CaCO}_{3}$ [261], together with a shift in the $T_{\mathrm{g}}$ towards higher temperatures. The largest increase in $T_{\mathrm{g}}$, up to $27^{\circ} \mathrm{C}$ at $4 \mathrm{wt} . \%$ loading was reported by Zheng et al. [263] for composites with silane-modified $\mathrm{Al}_{2} \mathrm{O}_{3}$, ascribed to a strong nanoparticle-matrix interfacial adhesion that hinders the chain motion in the amorphous region.

For other nanoparticle fillers in PA-6 the mechanical properties data available is diverse. Both Young's modulus and tensile strength increased up to 12 and $14 \%$, respectively, with only $0.01 \mathrm{wt} \%$ loading of fullerene $\mathrm{C}_{60}$ [265], and was ascribed to the formation of stereocomplexes that act as microfibers in the matrix. Similar enhancements were obtained for the same amount of a mixture of fullerene materials $\left(\mathrm{C}_{60} / \mathrm{C}_{70}\right)$ [265]. The incorporation of low contents $(\leq 2 \mathrm{wt} . \%)$ of untreated $\mathrm{SiO}_{2}$ $[149,172,173,264]$ also resulted in significant increases in the tensile properties, explained in terms of the nucleating effect of these nanofillers that promote the formation of the $\alpha$-phase. However, higher $\mathrm{SiO}_{2}$ concentrations led to a gradual decrease in properties due to particle agglomeration. The addition of silane [262] or acid-treated silica $[85,173]$ slightly improved the tensile strength of PA6 albeit provoking strong enhancements in toughness, since surface modification hindered the development of $\alpha$-crystallites and favored the formation of less ordered $\gamma$-crystals. Ou et al. [85] found a maximum increase in strength, ductility and toughness of PA-6/amino acid $\left(\mathrm{NH}_{2}-\right.$ A)-modified $\mathrm{SiO}_{2}$ nanocomposites at an optimum filler concentration of $5 \mathrm{wt} . \%$. The deterioration of properties at higher loadings arose from a ductile-to-brittle transition due to the formation of aggregates. Extraordinary improvements in the tensile properties of up to a 7 -fold increase at 4 wt.\% loading were reported by Sonawane et al. [176] for PA- $6 / \mathrm{CaCO}_{3}$ composites, attributed to a very uniform and fine nanofiller dispersion throughout the matrix that favors the formation of small spherulites, and by Rangari et al. [217] for PA-6/ $\mathrm{Si}_{3} \mathrm{~N}_{4}$ (1 wt.\%) composite fibers, ascribed to the high load-bearing capability of these nanoparticles and their alignment during extrusion in the fiber direction. 

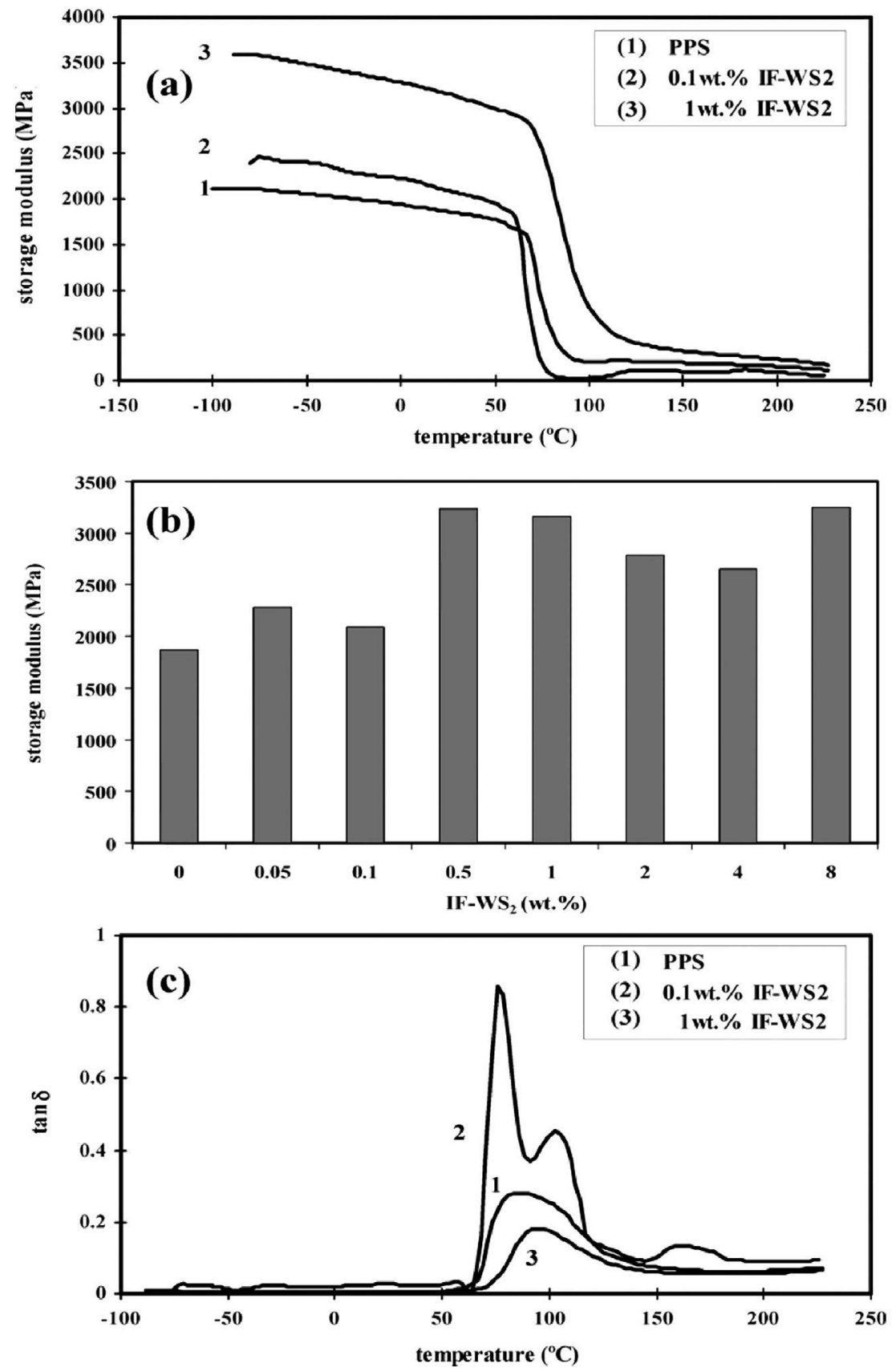

Fig. 31. Dynamic mechanical analysis of PPS/IF-WS 2 nanocomposites: (a) storage modulus versus temperature, (b) room temperature storage modulus

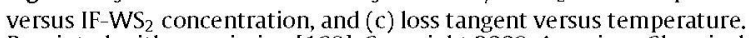

Reprinted with permission [168]. Copyright 2009, American Chemical Society.

Noticeable enhancements in the flexural behavior were found for nanocomposites incorporating $2-8 \mathrm{wt} . \%$ silanetreated $\mathrm{TiO}_{2}[174]$.

\subsubsection{Poly(phenylene sulphide)}

PPS exhibits good mechanical properties; however, certain applications of this polymer have been limited because of its relatively low $T_{g}$ compared to its high $T_{\mathrm{m}}$, its brittleness and relatively low strength. To overcome these limitations, particularly to enhance toughness, several studies have been reported on the incorporation of nanofillers, including IF-WS 2 , Table 9 .

IF-WS 2 nanoparticles appear to be highly suitable for simultaneously enhancing the stiffness, strength and toughness of PPS, as recently reported by Diez-Pascual et al. [266]. Their high stiffness [38] provides a strong reinforcement effect, and their lubricant character reduces the viscosity of the matrix, enabling a very homogenous dispersion within the polymer, hence large nanoparticle-matrix interfacial area. This results in notable increases in the 
Young's modulus and tensile strength, without sacrificing ductility, at 2 wt.\% loading. Their efficiency as reinforcing elements was also demonstrated by DMA measurements, Fig. 31 [168]. The incorporation of very low IF-WS 2 contents $\leq 0.1$ wt.\% slightly increased the room temperature storage modulus, Fig. $31 \mathrm{a}$ and b, provoking a decrease in $T_{\mathrm{g}}$, Fig. $31 \mathrm{c}$, while the addition of higher loadings led to strong improvements in modulus in the range of $40-75 \%$, and significant increments in $T_{\mathrm{g}}$. These differences are related to the crystallization behavior of these nanocomposites described in Section 4.3. High IF-WS 2 loadings act as NAs, and favor the formation of small imperfect crystals that are known to increase the rigidity of crystalline PPS [267]. In contrast, very small amounts of IF-WS 2 reduce the crystallization rate, contributing to the formation of a more amorphous phase, thereby leading to a decrease in $T_{\mathrm{g}}$. It is interesting to note that the porcentual improvements in the storage modulus of PPS/IF-WS 2 nanocomposites are noticeably higher than those achieved in other reported IF-PNCs (e.g., with iPP, nylon- 6 and PEEK), suggesting the presence of specific polymer-filler interactions in the case of PPS. The molecular nature of these interactions are still not understood, but they may be associated with the presence of outer $S$ atoms on the IF nanoparticles, and more work is required in order to explain this phenomenon.

Composites incorporating $3 \mathrm{wt} \%$ epoxy-g-SiO 2 prepared via ball-milling and melt-blending [164] exhibited $\sim 73 \%$ increase in impact strength compared to that of the neat matrix, ascribed to a very fine nanofiller dispersion and a strong $\mathrm{SiO}_{2}$-PPS interfacial bonding enhanced by crosslinking at high temperature that allows energy dissipation. More interestingly, Wang et al. [268] reported a three-fold increase in toughness for $\mathrm{PPS} / \mathrm{CaCO}_{3}$ nanocomposites with the same filler content, attributed to a cavitation-induced shear deformation mechanism promoted by the $\mathrm{CaCO}_{3}$ combined with a reduction in the crystallinity of the matrix. However, the addition of other nanoparticles such as $\mathrm{Al}_{2} \mathrm{O}_{3}$ [269], $\mathrm{TiO}_{2}$, and $\mathrm{CuO}$ [270] led to a decrease in the flexural strength of PPS due to a weakening of the matrix induced by the presence of a heterogeneous phase.

\subsubsection{Poly(ether ether ketone)}

PEEK exhibits outstanding mechanical properties, including flexural, modulus and strength. To further improve its mechanical performance, particularly for applications that have to withstand harsh environmental conditions, nanoscale inorganic fillers have been dispersed within this thermoplastic matrix [152,159].

The properties of PEEK/IF-WS 2 composites prepared via melt-blending, after a pre-dispersion step based on ballmilling and ultrasonication in organic solvent, were studied using both static and dynamic mechanical tests [57]. The stress-strain curves, Fig. 32a revealed that the incorporation of IF-WS 2 enhances the Young's modulus of the matrix by $\sim 37 \%$ at 2 wt.\% loading, Fig. 32b). The elastic deformation transfer taking place at the PEEK-nanoparticle interface explains the increased stiffness. This increment is larger than that of PEEK nanocomposites incorporating similar amounts of other inorganic nanoparticles such as $\mathrm{SiO}_{2}[152,159,218], \mathrm{Al}_{2} \mathrm{O}_{3}[152,271]$ or $\mathrm{TiO}_{2}[272,273]$,
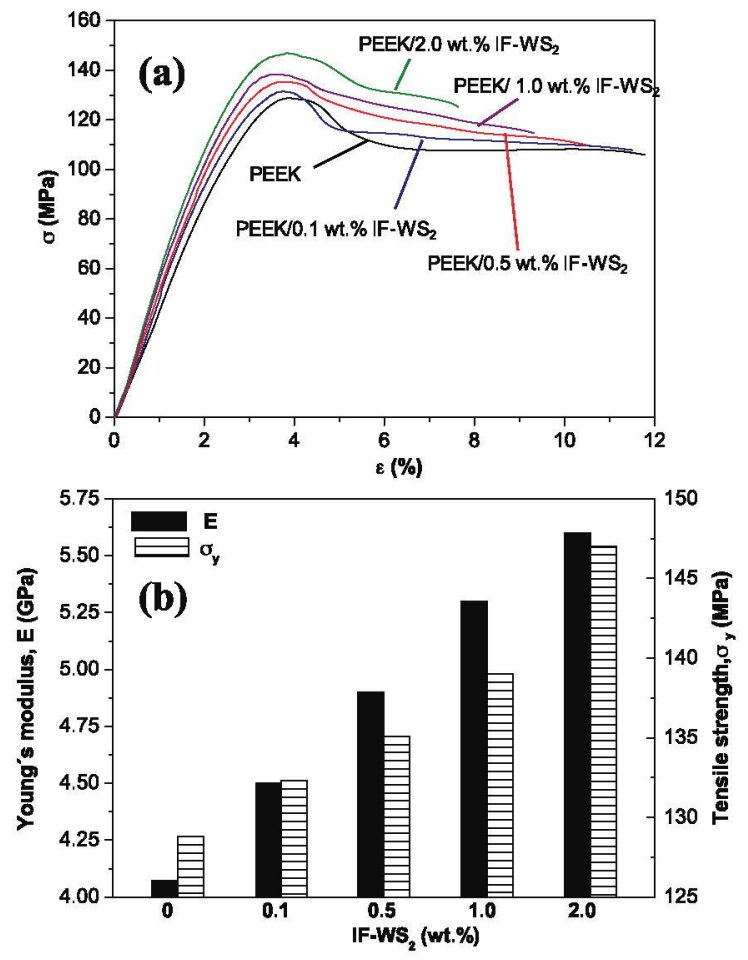

Fig. 32. (a) Representative room temperature stress-strain curves for PEEK based nanocomposites with different IF-WS ${ }_{2}$ loadings and (b) Young's modulus and tensile strength as a function of nanoparticle content.

Adapted with permission [57]. Copyright 2010, American Chemical Society.

Table 10 , where addition of $10 \mathrm{wt} . \% \mathrm{SiO}_{2}[152], 3 \mathrm{wt} . \% \mathrm{Al}_{2} \mathrm{O}_{3}$ [271] or $29 \mathrm{wt} . \% \mathrm{TiO}_{2}$ [272] was required to attain similar modulus improvements. PEEK/IF-WS 2 nanocomposites showed a non-linear growth of the Young's modulus with the filler content, the increase being more pronounced at low concentrations, in agreement with the behavior found for $\mathrm{SiO}_{2}$-reinforced composites prepared via ball-milling and compression molding [218]. However, Kuo et al. [152] and Lai et al. [159] found a quasi linear rise in modulus for up to $10 \mathrm{wt} . \% \mathrm{SiO}_{2}$, with higher increments for nanocomposites incorporating SA-modified silica as a result of the higher nanofiller density and lower tendency to agglomerate. With regard to the tensile strength, a progressive enhancement with increasing IF-WS ${ }_{2}$ content up to $14 \%$ at 2 wt.\% loading was found, Fig. 32b. However, for PEEK composites reinforced with $\mathrm{Al}_{2} \mathrm{O}_{3}$ or unmodified or surface modified $\mathrm{SiO}_{2}[152,159]$, the strength reached a maximum value at 5 and 7.5 wt.\% loading, respectively, and then decreased considerably, contrary to the trend observed for the modulus, and was ascribed to local particle clustering and premature failure. Nanoparticle aggregation was also found in $\mathrm{PEEK} / \mathrm{CaCO}_{3}$ nanocomposites [274], where the nanoparticles had very little influence on the strength of the polymer.

The strain at break was reported to decrease slightly up to $0.5 \mathrm{wt} . \%$ IF-WS 2 [57], although it dropped by $\sim 36 \%$ for the nanocomposite containing 2 wt.\% loading, indicating that larger amounts of these nanoparticles restrict the 
ductile flow of the matrix. Nevertheless, this restriction is less important than that observed upon addition of the same amount of silica ( $70 \%$ ) [218] or alumina ( $40 \%)$ [152], where the particle agglomeration clearly perturbs plastic deformation of the polymer chains. In the case of nanocomposites incorporating modified silica [159] the decrease in tensile elongation of $\sim 50 \%$ was found to be smaller than that of the unmodified counterparts due to the more homogenous filler dispersion.

The area under the tensile curve is a measure of the toughness of the system. In nanocomposites containing IF-WS ${ }_{2}$, this area was maintained up to $0.5 \mathrm{wt} \%$, and then decreased slightly, Fig. 32a. Overall, the addition of low contents of IF-WS 2 to PEEK provides a good balance between stiffness and toughness, while other inorganic nanoparticles generally lead to an improvement in the tensile modulus with the sacrifice of ductility.

It is well known that the size, state of nanofiller dispersion and its interfacial adhesion with the matrix strongly condition the rate of energy absorption, hence the impact properties of PNCs [5]. The impact strength of PEEK increased slightly at low IF-WS ${ }_{2}$ contents $(\leq 0.5 \mathrm{wt} . \%)$, while higher loadings led to a moderate decrease in toughness. Stronger decreases were found for other PEEK based nanocomposites, such as those filled with $\mathrm{CaCO}_{3}$ (5-30wt.\% loading) [274], where the presence of small aggregates provoked stress concentration sites, hence accelerating the propagation of cracks. In contrast, remarkable increases were reported for $\mathrm{TiO}_{2}(2.6-18 \mathrm{wt} . \%)$ [273] and $\mathrm{Al}_{2} \mathrm{O}_{3}$ (1-3wt.\%) [271] reinforced PEEK composites, ascribed to strong nanofiller-matrix interactions. Guoliang et al. [275] studied the impact strength and fracture mechanism of PEEK $/ \mathrm{Al}_{2} \mathrm{O}_{3}$ nanocomposites prepared by different dispersing methods and found that the presence of a coupling agent increased the toughness of the material, particularly in the case of titanate that led to an unprecedented eight-fold increase in the impact resistance of PEEK at $5 \mathrm{wt} \% \mathrm{Al}_{2} \mathrm{O}_{3}$, and doubled that of the corresponding composite without the coupling agent. The generation of a ductile interface layer between the surface of the particles and the coupling agent was responsible for the improvement in the absorption of impact energy. The stiffness of PEEK based nanocomposites has also been evaluated through DMA. Increasing IF-WS 2 loadings led to progressive enhancements in the storage modulus, up to $33 \%$ at room temperature and $2 \mathrm{wt}$ \% loading [57]. This increment is larger than that obtained by the addition of the same amount of unmodified or surface-modified silica [159], and only slightly smaller than that found for nanocomposites of PEEK/AIN at 20 wt.\% [276] or PEEK/TiO ${ }_{2}$ at 25 wt.\% [273]. The reinforcing efficiency of IF-WS $\mathrm{W}_{2}$ is considerably more pronounced at temperatures below $T_{\mathrm{g}}$, in agreement with the behavior found for PEEK filled with $\mathrm{SiO}_{2}$ [159] or $\mathrm{TiO}_{2}$ [273], where the differences between the composite modulus and that of the neat matrix become negligible at temperatures above $200^{\circ} \mathrm{C}$. In contrast, for PEEK/AlN and PEEK/ $/ \mathrm{Al}_{2} \mathrm{O}_{3}$ nanocomposites Goyal et al. [276,277] found larger stiffness increments at temperatures above $T_{\mathrm{g}}$. In particular, the addition of $70 \mathrm{wt} . \%$ AlN led to about five-fold and fourteen-fold increases in the storage modulus of the polymer at 30 and $200^{\circ} \mathrm{C}$, respectively. In the composite with the same amount of $\mathrm{Al}_{2} \mathrm{O}_{3}$ the increments were about two and six-fold, respectively, for the temperatures indicated. The extraordinary enhancement in the former composites was attributed to a very strong AlNmatrix adhesion that restricted segmental motion of the polymer chains.

Hou et al. [58] reported the use of IF-WS 2 nanoparticles to prepare self-lubricating PEEK-based nanocomposite coatings by an aerosol-assisted deposition process, and investigated their mechanical properties through nanoindentation tests. The Young's modulus increased with IF-WS 2 concentration, by up to $\sim 61 \%$ at $20 \mathrm{wt}$. loading. The stiffness enhancement was attributed to the reinforcing effect of the nanoparticles, combined with the increase in the crystallinity of the matrix.

Finally, it is clear that the improved mechanical properties of these nanocomposite coatings are influenced not only by the size and particle concentration, but also by the nature of the polymer-nanoparticle interface. However, no clear structural or spectroscopic evidence has been presented to date. FTIR analysis of PEEK/IF nanocomposite coatings have shown fundamentally identical spectra in the nanocomposites compared to the original PEEK powder, implying that the chemical structure of the PEEK matrix is preserved [58]. The only difference described was a slight shift and broadening of a band in PEEK from 1159 to $1153 \mathrm{~cm}^{-1}$ in the nanocomposite coating, which was attributed to the presence of sulphur-containing groups at the interface of the IF nanoparticles and the PEEK matrix, an assertion that is yet to be corroborated. It is clear that further work incorporating careful spectroscopic measurements needs to be undertaken on this and other nanocomposite systems in order to throw more light on the interaction mechanisms involved.

\subsection{Tribological properties}

The incorporation of hard nanoscale particles is a typical strategy used to improve the tribological properties of polymers, mainly the coefficient of friction (COF) and wear rate. The nanoparticles exhibit some advantages compared to conventional microfillers, such as higher SSA (hence stronger adhesion), lower abrasiveness due to a reduced angularity, enhanced strength, modulus and toughness, and high effectivity at very low loadings. The wear resistance of polymer composites depends on the ability to form a thin, uniform and adherent transfer film that prevents direct contact between a polymer pin and a metal counterface, therefore avoiding abrasive action [268]. By adding nanoparticles such as $\mathrm{TiO}_{2}, \mathrm{SiC}, \mathrm{ZrO}_{2}, \mathrm{SiO}_{2}, \mathrm{Si}_{3} \mathrm{~N}_{4}, \mathrm{ZnO}$ and $\mathrm{CuO}$, the transfer film can be strengthened due to anchoring or tribochemical reaction, resulting in reduced wear The particle size, shape, dispersion, orientation and concentration exert a powerful influence on the tribological performance of the nanocomposites [278], and smaller and well-dispersed particles lead to a better property profile under dry sliding wear conditions.

Recently, the efficiency of IF-WS 2 as a solid lubricant additive for improving the tribological properties of epoxy [59] and thermoplastic polymers $[58,167]$ has been demonstrated, and was ascribed to their high stiffness and 
Table 11

Tribological properties of thermoplastic/IF-WS 2 and different nanoparticle-filled thermoplastic nanocomposites.

\begin{tabular}{|c|c|c|c|c|c|c|c|c|}
\hline Matrix & $\begin{array}{l}\text { Nanofiller (Particle } \\
\text { size/nm) }\end{array}$ & $\begin{array}{l}\text { Nanofiller content } \\
\text { (wt.\%) }\end{array}$ & $\begin{array}{l}\text { Processing } \\
\text { method }\end{array}$ & $\mathrm{COF}$ & $\mathrm{COF}_{\mathrm{p}} / \mathrm{COF}_{\mathrm{c}}$ & $K\left(\times 10^{-6} \mathrm{~mm}^{3} / \mathrm{Nm}\right)$ & $K_{\mathrm{p}} / K_{\mathrm{c}}$ & Ref. \\
\hline $\mathrm{PP}$ & IF-WS $2(40-180)$ & 1 & $\mathrm{mb}$ & 0.17 & 1.35 & 221 & 2.86 & [244] \\
\hline $\mathrm{PP}$ & $\mathrm{TiO}_{2}(50)$ & $\begin{array}{l}10 \\
40\end{array}$ & knd + minj & - & - & $\begin{array}{l}210 \\
126\end{array}$ & $\begin{array}{l}3 \\
5\end{array}$ & [280] \\
\hline PP & $\begin{array}{l}\text { TMPM-modified } \\
\mathrm{SiO}_{2}(12)\end{array}$ & $\begin{array}{l}9.6 \\
19.1 \\
29.1 \\
48\end{array}$ & $\mathrm{mb}$ & $\begin{array}{l}- \\
- \\
-\end{array}$ & $\begin{array}{l}- \\
- \\
-\end{array}$ & $\begin{array}{l}85 \\
\\
72 \\
59 \\
44\end{array}$ & $\begin{array}{l}1.29 \\
1.53 \\
1.86 \\
2.5\end{array}$ & [281] \\
\hline $\mathrm{PP}$ & $\mathrm{PS}-\mathrm{g}-\mathrm{SiO}_{2}(7)$ & $\begin{array}{l}0.8 \\
1.3 \\
4 \\
6.6\end{array}$ & $m b+c m$ & $\begin{array}{l}0.88 \\
0.73 \\
0.93 \\
0.80\end{array}$ & $\begin{array}{l}0.97 \\
1.17 \\
0.92 \\
1.06\end{array}$ & $\begin{array}{l}57.4 \\
39.5 \\
44.1 \\
56.9\end{array}$ & $\begin{array}{l}1.77 \\
2.57 \\
2.3 \\
1.78\end{array}$ & [282] \\
\hline PA6 & IF-WS $2(40-180)$ & 1 & $\mathrm{mb}$ & 0.07 & 1.33 & - & - & {$[167]$} \\
\hline PA6 & $\mathrm{SiO}_{2}(10-20)$ & $\begin{array}{r}2 \\
14\end{array}$ & $\mathrm{sb}+\mathrm{cm}$ & $\begin{array}{l}0.2 \\
0.4\end{array}$ & $\begin{array}{l}2.25 \\
1.12\end{array}$ & $\begin{array}{r}0.2 \\
28.1\end{array}$ & $\begin{array}{r}265 \\
1.89\end{array}$ & [284] \\
\hline PA6 & $\begin{array}{l}\text { APTES-modified } \\
\mathrm{Al}_{2} \mathrm{O}_{3}(10)\end{array}$ & $\begin{array}{l}3 \\
5\end{array}$ & isp & $\begin{array}{l}0.17 \\
-\end{array}$ & $\begin{array}{l}0.94 \\
-\end{array}$ & $\begin{array}{l}720 \\
930\end{array}$ & $\begin{array}{l}2.36 \\
1.82\end{array}$ & [285] \\
\hline PA6 & $\mathrm{Cu}$ & $\begin{array}{l}1.5 \\
3 \\
5\end{array}$ & $\mathrm{~cm}$ & $\begin{array}{l}0.52 \\
0.52 \\
0.52\end{array}$ & $\begin{array}{l}1 \\
1 \\
1\end{array}$ & $\begin{array}{r}150 \\
85.0 \\
60.0\end{array}$ & $\begin{array}{l}1.74 \\
2.36 \\
3.34\end{array}$ & [286] \\
\hline PPS & IF-WS $2(40-180)$ & $\begin{array}{l}0.5 \\
1 \\
2\end{array}$ & $\mathrm{mb}$ & $\begin{array}{l}0.35 \\
0.29 \\
0.26\end{array}$ & $\begin{array}{l}1.14 \\
1.38 \\
1.54\end{array}$ & $\begin{array}{l}68.3 \\
36 \\
24.9\end{array}$ & $\begin{array}{c}4.54 \\
8.61 \\
12.4\end{array}$ & [287] \\
\hline PPS & $\mathrm{Al}_{2} \mathrm{O}_{3}(33)$ & $\begin{array}{r}1.5 \\
2.9 \\
4.4 \\
7.3 \\
14.6\end{array}$ & $m b+c m$ & $\begin{array}{l}0.5 \\
0.5 \\
0.48 \\
0.5 \\
0.42\end{array}$ & $\begin{array}{l}0.92 \\
0.92 \\
0.96 \\
0.92 \\
1.10\end{array}$ & $\begin{array}{l}17.2 \\
12.0 \\
22.2 \\
33.5 \\
75.2\end{array}$ & $\begin{array}{l}1 \\
1.42 \\
0.77 \\
0.5 \\
0.22\end{array}$ & [269] \\
\hline PPS & $\mathrm{TiO}_{2}(30-50)$ & $\begin{array}{l}3.1 \\
6 \\
8.8 \\
14.2\end{array}$ & $m b+c m$ & $\begin{array}{l}0.35 \\
0.37 \\
0.45 \\
0.49\end{array}$ & $\begin{array}{l}1.23 \\
1.16 \\
0.96 \\
0.88\end{array}$ & $\begin{array}{r}13 \\
8 \\
22 \\
36\end{array}$ & $\begin{array}{l}1.19 \\
2 \\
0.73 \\
0.45\end{array}$ & [270] \\
\hline PPS & $\mathrm{ZnO}(30-50)$ & $\begin{array}{c}4 \\
7.8 \\
17.9\end{array}$ & $\mathrm{mb}+\mathrm{cm}$ & $\begin{array}{l}0.39 \\
0.42 \\
0.46\end{array}$ & $\begin{array}{l}1.1 \\
1.02 \\
0.93\end{array}$ & $\begin{array}{l}45.5 \\
53.8 \\
77.2\end{array}$ & $\begin{array}{l}0.35 \\
0.3 \\
0.21\end{array}$ & [270] \\
\hline PPS & $\mathrm{CuO}(30-50)$ & $\begin{array}{r}4.6 \\
8.8 \\
12.8 \\
34.5\end{array}$ & $\begin{array}{l}m b+c m \\
m b+c m\end{array}$ & $\begin{array}{l}0.41 \\
0.39 \\
0.37 \\
0.34\end{array}$ & $\begin{array}{l}1.04 \\
1.1 \\
1.16 \\
1.26\end{array}$ & $\begin{array}{c}4.89 \\
4.6 \\
9.49 \\
11.6\end{array}$ & $\begin{array}{l}3.9 \\
4.15 \\
2.01 \\
1.65\end{array}$ & [275] \\
\hline PPS & $\operatorname{SiC}(30-50)$ & $\begin{array}{c}4.6 \\
11\end{array}$ & & $\begin{array}{l}0.41 \\
0.47\end{array}$ & $\begin{array}{l}1.05 \\
0.91\end{array}$ & $\begin{array}{l}31 \\
48.4\end{array}$ & $\begin{array}{l}0.52 \\
0.33\end{array}$ & [270] \\
\hline PEEK & IF-WS $2(40-180)$ & $\begin{array}{l}0.1 \\
0.5 \\
1\end{array}$ & $\mathrm{bml}+\mathrm{mb}$ & $\begin{array}{l}0.31 \\
0.27 \\
0.25\end{array}$ & $\begin{array}{l}1.17 \\
1.35 \\
1.46\end{array}$ & $\begin{array}{l}9.5 \\
4.8 \\
2 .\end{array}$ & $\begin{array}{l}2.63 \\
5.21 \\
9.26\end{array}$ & [287] \\
\hline PEEK & IF-WS $2(80-300)$ & $\begin{array}{r}2 \\
5 \\
10\end{array}$ & $\mathrm{jml}+\mathrm{cm}$ & $\begin{array}{l}0.4 \\
0.38 \\
0.36\end{array}$ & $\begin{array}{l}1.09 \\
1.15 \\
1.2\end{array}$ & $\begin{array}{r}3.05 \\
8.69 \\
81.3\end{array}$ & $\begin{array}{l}4.62 \\
1.61 \\
0.17\end{array}$ & [289] \\
\hline PEEK $^{\mathrm{a}}$ & IF-WS $(80-220)$ & $\begin{array}{l}1 \\
2.5 \\
5 \\
10 \\
20\end{array}$ & aed & $\begin{array}{l}0.25 \\
0.15 \\
0.18 \\
0.19 \\
0.2\end{array}$ & $\begin{array}{l}1.7 \\
2.83 \\
2.36 \\
2.23 \\
2.12\end{array}$ & $\begin{array}{l}- \\
- \\
- \\
- \\
-\end{array}$ & $\begin{array}{l}- \\
- \\
- \\
- \\
-\end{array}$ & [58] \\
\hline PEEK & $\mathrm{C}_{60}$ & $\begin{array}{r}2 \\
5 \\
10\end{array}$ & $\mathrm{jml}+\mathrm{cm}$ & $\begin{array}{l}0.55 \\
0.49 \\
0.59\end{array}$ & $\begin{array}{l}0.79 \\
0.88 \\
0.74\end{array}$ & $\begin{array}{l}5.65 \\
0.89 \\
0.54\end{array}$ & $\begin{array}{c}2.49 \\
15.8 \\
26.2\end{array}$ & [289] \\
\hline
\end{tabular}


Table 11 (Contimued)

\begin{tabular}{|c|c|c|c|c|c|c|c|c|}
\hline Matrix & $\begin{array}{l}\text { Nanofiller (Particle } \\
\text { size/nm) }\end{array}$ & $\begin{array}{l}\text { Nanofiller content } \\
\text { (wt.\%) }\end{array}$ & $\begin{array}{l}\text { Processing } \\
\text { method }\end{array}$ & $\mathrm{COF}$ & $\mathrm{COF}_{\mathrm{p}} / \mathrm{COF}_{\mathrm{c}}$ & $K\left(\times 10^{-6} \mathrm{~mm}^{3} / \mathrm{Nm}\right)$ & $K_{\mathrm{p}} / K_{\mathrm{c}}$ & Ref. \\
\hline \multirow[t]{3}{*}{ PEEK } & \multirow[t]{3}{*}{$\mathrm{CNO}$} & 2 & \multirow[t]{3}{*}{$\mathrm{jml}+\mathrm{cm}$} & 0.65 & 0.67 & 2.99 & 4.71 & \multirow[t]{3}{*}{ [289] } \\
\hline & & 5 & & 0.49 & 0.89 & 2.16 & 6.53 & \\
\hline & & 10 & & 0.44 & 0.99 & 3.39 & 4.16 & \\
\hline \multirow[t]{6}{*}{ PEEK } & \multirow[t]{6}{*}{$\mathrm{Al}_{2} \mathrm{O}_{3}(39)$} & 1.2 & \multirow[t]{6}{*}{$\mathrm{ult}+\mathrm{cm}$} & 0.6 & 0.93 & 6 & 1.6 & \multirow[t]{6}{*}[277]{} \\
\hline & & 2.5 & & 0.58 & 0.97 & 2.8 & 3.4 & \\
\hline & & 5 & & 0.55 & 1.02 & 3.2 & 2.98 & \\
\hline & & 10 & & 0.52 & 1.08 & 13 & 0.73 & \\
\hline & & 20 & & 0.48 & 1.16 & 21 & 0.45 & \\
\hline & & 30 & & 0.45 & 1.24 & 35 & 0.27 & \\
\hline \multirow[t]{4}{*}{ PEEK } & \multirow[t]{4}{*}{$\mathrm{SiO}_{2}(13)$} & 1 & \multirow[t]{4}{*}{$\mathrm{blm}+\mathrm{cm}$} & 0.48 & 0.98 & 13 & 2.15 & \multirow[t]{4}{*}{ [218] } \\
\hline & & 2.1 & & 0.43 & 1.09 & 10 & 2.8 & \\
\hline & & 4.2 & & 0.51 & 0.92 & 21.5 & 1.3 & \\
\hline & & 8.4 & & 0.43 & 1.09 & 20 & 1.4 & \\
\hline \multirow[t]{6}{*}{ PEEK } & \multirow{6}{*}{$\mathrm{SiO}_{2}(<100)$} & 0.5 & \multirow[t]{6}{*}{$\mathrm{cm}$} & 0.38 & 1 & 5.30 & 1.41 & \multirow[t]{6}{*}{ [292] } \\
\hline & & 1 & & 0.37 & 1.04 & 4.75 & 1.58 & \\
\hline & & 2.5 & & 0.30 & 1.27 & 3.00 & 2.5 & \\
\hline & & 5 & & 0.26 & 1.46 & 2.60 & 2.88 & \\
\hline & & 10 & & 0.22 & 1.73 & 3.1 & 2.42 & \\
\hline & & 15 & & 0.21 & 1.81 & 3.60 & 2.08 & \\
\hline \multirow[t]{6}{*}{ PEEK } & \multirow[t]{6}{*}{$\operatorname{SiC}(80)$} & 1 & \multirow[t]{6}{*}{$\mathrm{cm}$} & 0.36 & 1.06 & 5 & 1.46 & \multirow[t]{6}{*}{ [293,294] } \\
\hline & & 2.5 & & 0.32 & 1.19 & 4.45 & 1.64 & \\
\hline & & 5 & & 0.31 & 1.26 & 3.5 & 2.09 & \\
\hline & & 10 & & 0.27 & 1.41 & 3.75 & 1.95 & \\
\hline & & 15 & & 0.22 & 1.73 & 3.85 & 1.9 & \\
\hline & & & & 0.2 & 1.90 & 9.75 & 0.75 & \\
\hline \multirow[t]{5}{*}{ PEEK } & \multirow[t]{5}{*}{$\mathrm{ZrO}_{2}(10)$} & 1 & \multirow[t]{5}{*}{$\mathrm{cm}$} & 0.33 & 1.15 & 6.4 & 1.16 & \multirow[t]{5}{*}{ [291] } \\
\hline & & 2.5 & & 0.32 & 1.19 & 5.95 & 1.24 & \\
\hline & & 5 & & 0.31 & 1.23 & 4.5 & 1.64 & \\
\hline & & 10 & & 0.3 & 1.26 & 5.55 & 1.33 & \\
\hline & & 15 & & 0.29 & 1.31 & 7.05 & 1.05 & \\
\hline PEEK & $\mathrm{Si}_{3} \mathrm{~N}_{4}(<50)$ & 0.5 & $\mathrm{~cm}$ & 0.37 & 1.03 & 4 & 1.87 & [290] \\
\hline & & 1 & & 0.37 & 1.04 & 3.5 & 2.14 & \\
\hline & & 2.2 & & 0.35 & 1.08 & 2.55 & 2.94 & \\
\hline & & 5 & & 0.29 & 1.31 & 2.55 & 2.94 & \\
\hline & & 10 & & 0.24 & 1.58 & 2.25 & 3.33 & \\
\hline & & 15 & & 0.23 & 1.65 & 3.5 & 2.14 & \\
\hline PEEK & $\mathrm{Si}_{3} \mathrm{~N}_{4}(<50)$ & 2.5 & ult $+\mathrm{cm}$ & 0.36 & 0.86 & 5.4 & 1.8 & [153] \\
\hline & & 5 & & 0.36 & 0.86 & 4.8 & 2 & \\
\hline & & 10 & & 0.41 & 0.76 & 4.2 & 2 & \\
\hline & & 20 & & 0.44 & 0.7 & 8.1 & 1 & \\
\hline
\end{tabular}

COF: Coefficient of friction; K: Wear rate; the subscripts p and c denote the polymer and composite, respectively.

Key: $\mathrm{mb}=$ melt blending; $\mathrm{cm}=$ compression molding; isp=in situ polymerization; bml=ball milling; aed=aerosol assisted deposition; ult = ultrasonication; Knd = kneading; $\operatorname{minj}=$ micro injection molding; $s b=$ solution blending; $\mathrm{jml}=\mathrm{jet}-\mathrm{milling}$.

APTES: Aminopropyltriethoxysilane; TMPM: trimethoxysilylpropylmethacrylate; PS: polystyrene; CNO: carbon nanoonion.

a Nanocomposite coating.

strength, chemical inertness and onion-like hollow cage structure that provides elasticity and an ultra-low COF. Rapoport et al. [279] proposed a rolling mechanism for the IF-WS $_{2}$ nanoparticles suggesting that they roll rather than slide between the two mating surfaces, effectively reducing the shear stress, COF and contact temperature. Moreover, the nanoparticles serve as spacers that prevent contact between the asperities of the composite surface and the counterpart. During friction, the IFs become flattened due to the high contact pressure and are broken into individual sheets because of the shear stress. Under severe contact conditions the gradual release of exfoliated external layers of the nanoparticles and their transfer to the contact surfaces has been reported to be the main friction mechanism when the distance between the rubbed surfaces is smaller than the nanoparticle dimensions [59]. In Table 11 the friction and wear properties of thermoplastic/IF-WS 2 nanocomposites are summarized and compared to the literature data for other nanoparticle-reinforced thermoplastic polymers.

\subsubsection{Polypropylene}

Neat iPP is relatively soft, limiting its application in tribological components. This inherent weakness can be partially overcome by the addition of $\mathrm{IF}_{-} \mathrm{WS} \mathrm{S}_{2}$, with a decrease of $\sim 26 \%$ in its COF at 1 wt.\% loading [244]. This can be explained in terms of the lubricating capacity of these nanoparticles, combined with the increase in thermal conductivity, leading to a lower temperature at the sliding contact. Further, this $\mathrm{iPP} / \mathrm{IF}-\mathrm{WS}_{2}$ nanocomposite 
demonstrated a reduction in wear rate of $\sim 65 \%$, attributed to the improvement in mechanical properties (modulus and strength) along with the aforementioned lubricant effect. This enhancement in wear resistance is quantitatively similar to that found for a $\mathrm{PP} / \mathrm{TiO}_{2}$ (10 wt.\%) nanocomposite [280], but larger than those obtained by the incorporation of $10-48 \mathrm{wt} \%$ silane-modified $\mathrm{SiO}_{2}$ [281] or 0.8-6.6 wt.\% PS-g-SiO 2 [282]. In fact, the IF-WS ${ }_{2}$ nanoparticles reduce the matrix viscosity and facilitate composite processing, and can be homogenously dispersed without the aid of surfactants or compatibilizing agents, being significantly more effective in enhancing tribological performance than other modified nanoparticles. Dasari et al. [283] investigated the role of SA as a surfactant on the tribological behavior of $\mathrm{PP} / \mathrm{CaCO}_{3}$ nanocomposites with various nanofiller loadings. The surfactant improved the dispersion of the nanoparticles, leading to composites with a lower $\mathrm{COF}$ and wear rate than those filled with untreated $\mathrm{CaCO}_{3}$. This was attributed to the formation of a thin, stable and uniform film on the counterpart surface in the presence of SA that decreased abrasion. In composites with either modified or unmodified nanoparticles, adhesive wear damage was observed, the magnitude of which was higher in those without modifier due to the presence of agglomerates. Thus, the best tribological performance was found for PP/SA-modified $\mathrm{CaCO}_{3}$ (15 wt.\%) and was comparable to that observed for $\mathrm{PPP} / \mathrm{IF}-\mathrm{WS}_{2}$ with only $1 \mathrm{wt} . \%$ nanofiller loading. In general, the wear resistance of PP based nanocomposites improves with increasing nanofiller content, since the nanoparticles are much harder than the polymer and resist abrasion, resulting in a more adhesive wear.

\subsubsection{Nylon-6}

Nylon- 6 has a high abrasion resistance and low COF that are crucial for applications such as brakes, bearings or gears. To further improve its tribological behavior different nanoparticles have been added to this thermoplastic. Recently, Naffakh et al. [167] found a $25 \%$ reduction in the COF of PA-6, approaching that of PTFE or UHMWPE, when $1 \mathrm{wt} . \%$ IF-WS 2 was incorporated, Fig. 5. This was attributed to a very efficient dispersion of the nanoparticles within the matrix and a decrease in the shearing force due to the low shear strength of the lubricant particles. A more significant decrease of $\sim 56 \%$ in the COF of PA- 6 was reported by Garcia et al. [284] upon incorporation of $2 \mathrm{wt} . \% \mathrm{SiO}_{2}$, where the surface of the nanocomposites was well protected by the transfer film on the counterface. At this low silica loading a reduction in wear rate by a factor of 265 was also observed, whereas the effect of higher nanoparticle contents was less pronounced. An analogous trend was reported by Zhao et al. [285] for PA-6/silane-modified $\mathrm{Al}_{2} \mathrm{O}_{3}$ composites, where the optimum reduction in wear rate was found at 3 wt.\% loading. However, an increase in the nanofiller concentration resulted in poorer performance due to nanoparticle agglomeration that generated stress concentration. The incorporation of $1.5-5 \mathrm{wt} . \% \mathrm{Cu}$ nanoparticles did not change the COF of PA-6, but the wear rate dropped more than threefold [286], attributed to an increase in the thermal conductivity of the material and the heat removal from local hot spots. These nanoparticles also inhibited thermooxidative degradation of the polymer
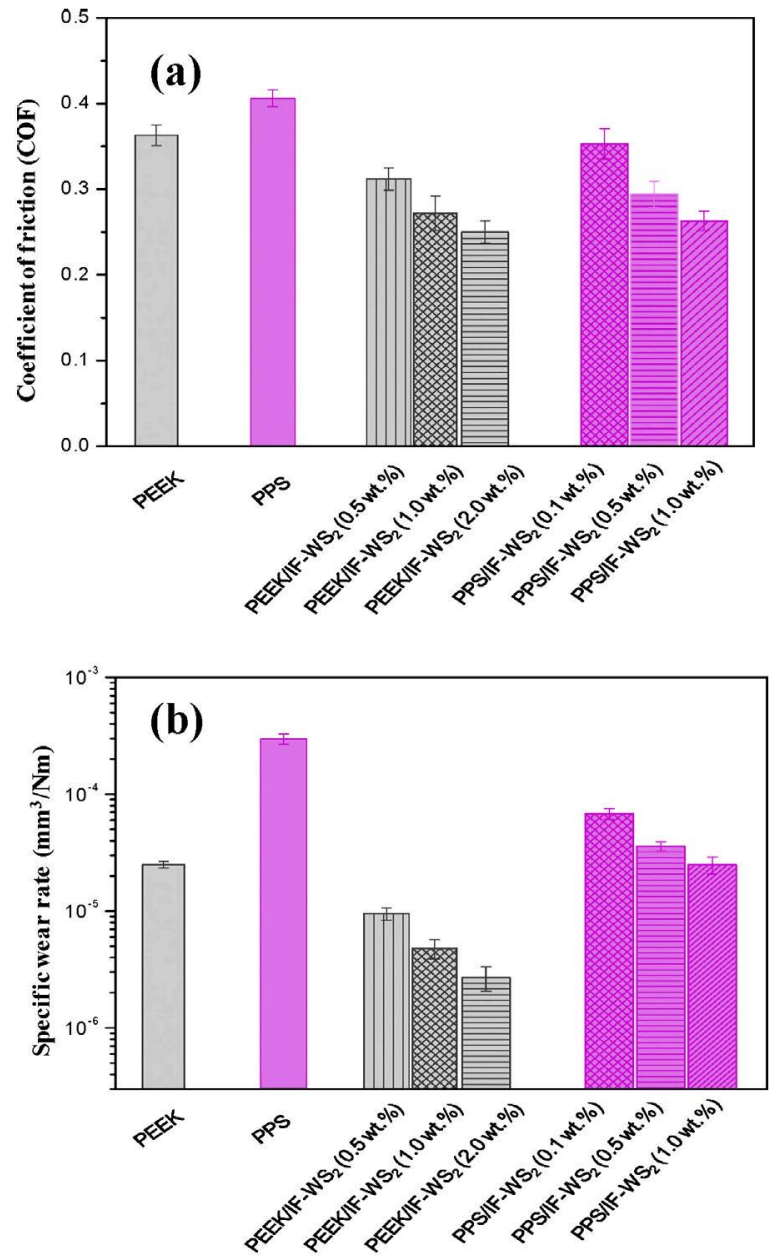

Fig. 33. Tribological properties of PEEK and PPS based nanocomposites reinforced with different IF-WS ${ }_{2}$ loadings. (a) Coefficient of friction. (b) Specific wear rate.

in the friction contact zone. Overall, it has been demonstrated that aggregated particles led to the worst wear resistance whereas systems with good nanoparticle-matrix interfacial adhesion and homogenous dispersion such as nylon-6/IF-WS 2 nanocomposites had improved tribological performance.

\subsubsection{Poly (phenylene sulphide)}

PPS is an attractive thermoplastic for tribological applications due to its good mechanical properties, dimensional stability, chemical and high-temperature resistance. The incorporation of IF-WS $2(0.5-2 \mathrm{wt} . \%)$ has been demonstrated to be effective for enhancement of its tribological behavior, Fig. 33) [287]. The largest improvements were encountered at $2 \mathrm{wt} \%$ loading, where the COF and wear rate dropped by factors of 1.5 and 12 , respectively. To investigate the role of the nanoparticles in modifying these properties, SEM was employed to analyze the wear tracks in both the polymer and its nanocomposites. The worn surface of neat PPS revealed the existence of microcracks, ploughing and a large amount of debris, and adhesive 
wear seemed to be the dominant mechanism. However, in the PPS/IF-WS 2 nanocomposites a smoother surface was observed, without signs of ploughing or cracking and with only a small amount of debris. The addition of these nanoparticles prevents adhesive wear and increases the surface hardness, providing resistance against the embedding of the hard asperities on the counterpart surface.

It can be noted that the improvements in wear resistance obtained by incorporating IF-WS 2 nanoparticles are considerably larger than those observed with other inorganic nanofillers such as $\mathrm{Al}_{2} \mathrm{O}_{3}$ [267], $\mathrm{CuO}, \mathrm{TiO}_{2}, \mathrm{ZnO}$ or $\mathrm{SiC}$ [268]. In PPS $/ \mathrm{Al}_{2} \mathrm{O}_{3}$ nanocomposites with 1.5-15 wt.\% loading [267], the COF observed in all cases was higher than that of PPS, except for that with $15 \mathrm{wt} . \%$ loading where it decreased by $\sim 8 \%$. Only the incorporation of $2.9 \mathrm{wt} . \% \mathrm{Al}_{2} \mathrm{O}_{3}$ led to a reduction in wear rate by a factor of 1.4 , and at higher loadings the reduced ability of the nanoparticles to anchor the transfer film to the counterface resulted in lower bond strength and higher wear rate. In addition, a strong dependence of the wear behavior on the nanoparticle composition was found, as reported by Bahadur et al. [268]. When $\mathrm{TiO}_{2}$ or $\mathrm{CuO}$ were used as nanofillers (3-9 wt.\%), a transfer film strongly adherent to the counterface was formed, decreasing the wear rate of PPS by up to factors of 2 and 4.1, respectively. In contrast, the addition of $\mathrm{ZnO}$ or $\mathrm{SiC}(4-18 w \mathrm{w} . \%)$ resulted in disruption of the transfer film leading to higher wear rates than that of the neat polymer.

\subsubsection{Poly(ether ether ketone)}

PEEK is regarded as one of the most promising polymeric materials for tribological applications due to its high service temperature, excellent chemical and abrasion resistance as well as good cohesive strength. The addition of nanoparticles to PEEK has generally led to an improvement in the tribological properties, similarly to the behavior observed in composites reinforced with microparticles [288]. Hou et al. [58] incorporated between 0.1 and $20 \mathrm{wt} \%$ IF-WS $_{2}$ into PEEK coatings and demonstrated that at $2.5 \mathrm{wt} . \%$ loading the COF decreased by $\sim 70 \%$, Fig. 34a. However, further increment in the nanoparticle content provoked only trivial improvement that was explained by two possibilities; firstly, the high loading of IF-WS 2 may influence the development of the transfer film during the wear process, and secondly, the tendency for nanoparticles agglomeration at higher concentrations resulting in a loss of the lubricating capacity. Therefore at relatively low loadings, between 2.5 and $5 \mathrm{wt} . \%$, IF-WS$_{2}$ nanoparticles can be considered efficient solid lubricants. SEM analysis of the worn surfaces revealed that for neat PEEK the coating had worn off and the substrate was exposed, Fig. 34b, while for PEEK/IF-WS 2 at $2.5 \mathrm{wt} . \%$ only slight wear had taken place, Fig. $34 \mathrm{c}$. The nanoparticles reduced plough and the adhesion between the sliding parts leading to an improved wear resistance due to the increase in hardness and Young's modulus as well as the decrease in the COF.

Mccook et al. [289] compared the tribological behavior of PEEK/IF-WS 2 (2-10 wt.\%) nanocomposites with those incorporating the same loading of $\mathrm{CNO}$ and $\mathrm{C}_{60}$. The $\mathrm{COF}$ decreased gradually with increasing IF-WS 2 content by up
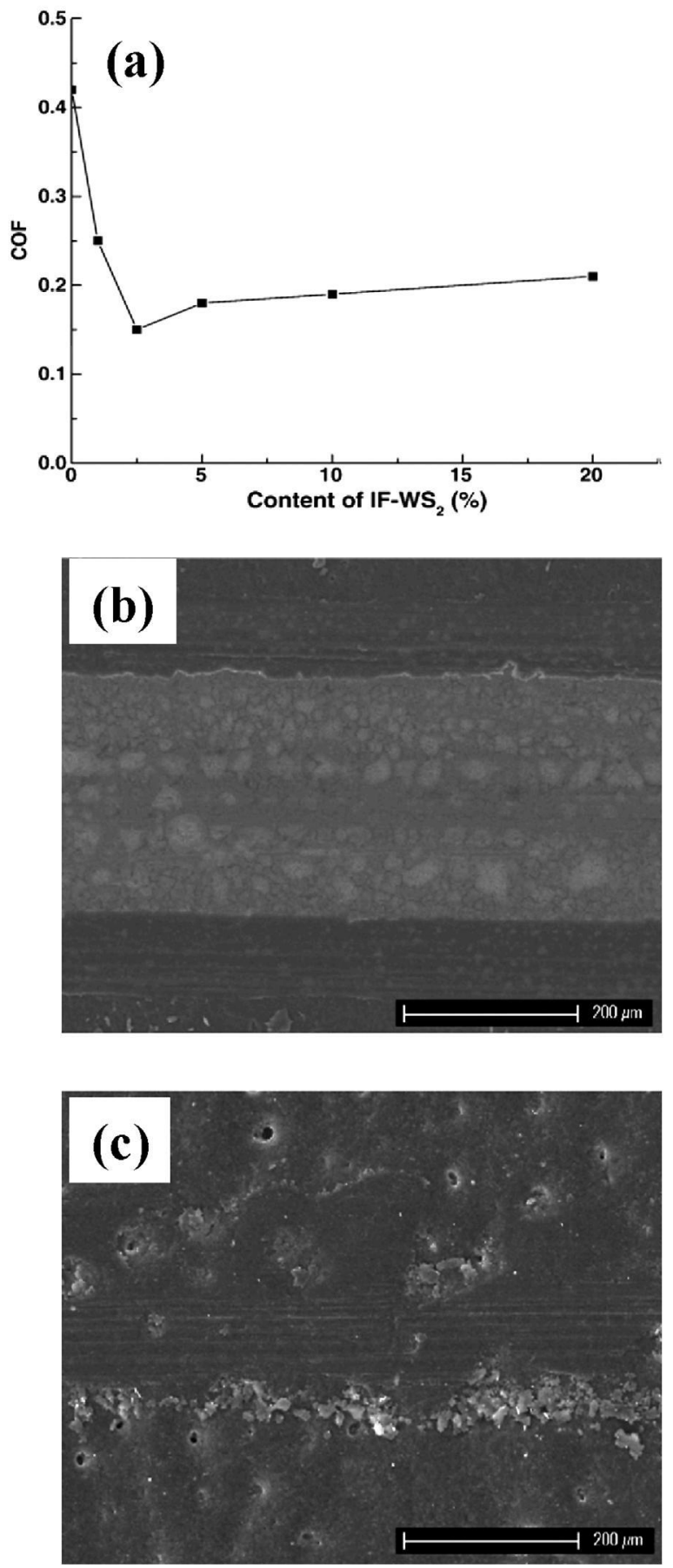

Fig. 34. (a) Coefficient of friction vs. IF-WS 2 concentration in PEEK based nanocomposite coatings. (b) and (c) SEM images of worn surfaces of PEEK and PEEK/IF-WS 2 (2.5 wt.\%) nanocomposite coatings, respectively. Reprinted with permission [58]. Copyright 2008, Elsevier.

to $16 \%$ at $10 \mathrm{wt} . \%$, whilst in the case of the carbon based nanofillers the COF was superior to that of the neat polymer for all concentrations tested. On the other hand, IF-WS was only effective in decreasing the wear rate at concentrations $\leq 5 \mathrm{wt} . \%$, whereas $\mathrm{C}_{60}$ and CNO improved the wear 
resistance over the whole concentration range, probably due to the increased shear strength of these composites.

Significant decreases in the COF in the range between 15 and $31 \%$ were also attained for PEEK/IF-WS 2 ( $0.1-1$ wt.\%) nanocomposites prepared via ball-milling and meltblending [287], Fig. 33. At a content of $1 \mathrm{wt} \%$ the reduction was considerably larger than that achieved with the equivalent amount of other nanoparticles such as $\mathrm{Al}_{2} \mathrm{O}_{3}$ [277], $\mathrm{Si}_{3} \mathrm{~N}_{4}$ [290] or $\mathrm{ZrO}_{2}$ [291], amongst others, and comparable to that obtained with $5 \mathrm{wt} . \% \mathrm{SiO}_{2}[292]$ or $\mathrm{SiC}[293,294]$. Wang et al. [290-294] investigated the tribological behavior of PEEK nanocomposites filled with $\mathrm{SiC}, \mathrm{SiO}_{2}, \mathrm{Si}_{3} \mathrm{~N}_{4}$ or $\mathrm{ZrO}_{2}$ as a function of nanofiller concentration. They found that the COF decreased sharply up to $7.5 \mathrm{wt} \%$, from a value of $\sim 0.38$ for neat PEEK to $0.2-0.3$ for the nanocomposites, whereas at higher concentrations it remained barely unchanged. Among the four aforementioned nanoparticles, $\mathrm{SiO}_{2}$ and $\mathrm{SiC}$ caused the largest reduction in this parameter. Nevertheless, Zhang et al. [218] found that the addition of $\mathrm{SiO}_{2}$ hardly affected the COF, whereas Goyal et al. [277] and Balaji et al. [153] reported an increase for $\mathrm{PEEK} / \mathrm{Al}_{2} \mathrm{O}_{3}$ and $\mathrm{PEEK} / \mathrm{Si}_{3} \mathrm{~N}_{4}$ nanocomposites, respectively, attributed to the higher contribution of the hard nanoparticles to the deformation component of friction during sliding, as demonstrated by the rise in surface roughness with increasing particle content, indicating a change from adhesion to abrasion in the wear mechanism.

Similar to the behavior found for the COF, the wear rate of PEEK/IF-WS 2 [287] decreased gradually with increasing nanoparticle loading by up to a factor of 9 at $1.0 \mathrm{wt} . \%$, Fig. 33. This improvement is remarkably larger than those reported for the incorporation of $1.0-15 \mathrm{wt} . \%$ of other inorganic nanoparticles [218,277,290-294], where a maximum wear resistance was obtained at higher optimum particle contents. For example, the wear rate of $\mathrm{PEEK} / \mathrm{Si}_{3} \mathrm{~N}_{4}$ [290] decreased strongly by a factor of 3.4 up to $10 \mathrm{wt} . \%$ loading, and then increased only slightly at higher concentrations. A similar trend was found for most of the PEEK based nanocomposites listed in Table 11. The increase in wear rate at higher loadings was more pronounced for composites filled with $\mathrm{ZrO}_{2}$ [291] or SiC [293,294], where it reached or even surpassed the value of the neat polymer, explained by the reduction in thickness, uniformity, toughness and adhesion of the transfer film to the substrate counterface during load application. An analogous behavior was reported by Zhang et al. [218] for $\mathrm{PEEK} / \mathrm{SiO}_{2}$, where the nanocomposite with $\sim 2 \mathrm{wt} . \% \mathrm{SiO}_{2}$ showed the lowest wear rate, but increasing the nanoparticle concentration resulted in the formation of agglomerates, and the observation of a more abrasive wear behavior. Taking into account the optimum loading of $\sim 7.5 \mathrm{wt} . \%$ reported for most of the nanoparticles, the optimum concentration found in that work [218] was notably lower and was explained by differences in the size and state of dispersion of the nanofillers. Goyal et al. [277] described a similar trend for PEEK/Al $\mathrm{A}_{2} \mathrm{O}_{3}$ nanocomposites, where the wear rate at $2.5 \mathrm{wt} . \% \mathrm{Al}_{2} \mathrm{O}_{3}$ experienced a more than three-fold decrease with respect to that of neat PEEK, but increased at higher loadings, despite the increased stiffness and hardness, indicating that the transfer film that developed during sliding plays a major role compared to that of the sample hardness.

\section{Novel dual filler strategies for hybrid thermoplastic polymer nanocomposites based on inorganic fullerene-like nanoparticles (IF-PNCs)}

PNCs incorporating inorganic nanoparticles such as the IFs discussed in this review can already be considered hybrid materials. However, the endless possibilities of combination of the wide range of nanomaterials available, as well as the incorporation of different scale fillers, provides the opportunity to tailor more sophisticated hybrid materials with complex architectures, interactions, morphology and functionality. This section addresses the combination of IFs with other organic micro-particles (nucleating agents, NAs), micro-fibers (carbon fibers, CFs) or nanofillers (carbon nanotubes, CNTs) in order to develop a new generation of hybrid thermoplastic polymer nanocomposites based on inorganic fullerene-like nanoparticles (IF-PNCs).

\subsection{IF-PNCS containing nucleating agents. The example of polypropylene}

The research and development of NAs, incorporated into thermoplastic polymers during processing, have been mainly industry driven and directed towards the improvement of the thermal, mechanical and optical properties of the polymer involved and/or to reduce production costs through cycle time efficiency. A great effort has been devoted to this during the last decades and this is still an area of substantial scientific and commercial interest [295-299].

NAs are specific additives that shorten the induction time of crystallization of a given polymer, providing foreign surfaces or nuclei that reduce the free energy of formation of a new polymer nucleus. This results in an increase of the rate of crystallization of the polymer and the formation of smaller crystals, which in turn will improve the optical and mechanical properties of the material. Although different mechanisms have been proposed in order to explain how these additives act, a number of authors have related nucleating ability to geometrical and dimensional similarities between the additive and the growing crystalline lattice which may promote epitaxial growth [300-302]. Other mechanisms such as self-seeding or chemical reactions have also been reported [303,304].

We have seen, and it is also well documented, that nanoreinforcements such as nano clays, $\mathrm{CaCO}_{3}$, silica, $\mathrm{CNTs}$, IFs, carbon nitrides and inorganic nanotubes among others, can exert a strong influence on the crystallization behavior of the matrix due to their nucleating ability depending on dispersion, polymer-filler interactions and crystallization conditions [93,126,188,194,305-309], which has a direct impact on the thermal, mechanical and tribological properties of the nanocomposites improving their performance. Recently a number of innovative approaches have incorporated nanoreinforcements simultaneously with other fillers, combining micro and nanoparticles [127,310] as well as dual-nanoparticles with different characteristics [198,311], in order to obtain new materials with superior properties taking advantage of filler synergy effects. Dual and competitive nucleating effects as well as changes 
in crystalline forms, which can be adjusted by the variation of filler proportions, have been reported. The addition of these mixed nucleating systems has opened new possibilities to develop multifunctional high performance materials.

As previously described in Section 4.3, IFs can act as NAs in the crystallization of thermoplastics such as iPP [93,188], PPS [226], PEEK [57], and PA-6 [144], and can influence the development of different crystalline forms and crystalline transformations. One of the main characteristics of iPP is its polymorphic behavior with four different crystal modifications, including the monoclinic $(\alpha)$, trigonal $(\beta)$, orthorhombic $(\gamma)$ and metastable mesomorphic forms [312-317]. While the $\alpha$-phase is the thermodynamically stable crystalline form, and hence the most commonly encountered modification of iPP, the lower $T_{\mathrm{m}}$ and density of the $\beta$-phase make this modification an interesting alternative to the $\alpha$-iPP for specific applications. The addition of specific agents that nucleate the $\alpha$-form can improve tensile and flexural behavior as well as the transparency of iPP, while $\beta$-nucleating agents can improve the impact strength and heat distortion temperature of iPP, albeit at the expense of stiffness [181,246,318-320]. In this respect, the combination of $\alpha$ and $\beta$-NAs is a common strategy employed to obtain controlled adjustment of stiffness and toughness of iPP [321-325]. In these systems it is of fundamental importance to identify which NA dominates the crystallization process and to understand which parameters affect the nucleation behavior.

The preparation of $\mathrm{iPP} / \mathrm{IF}-\mathrm{WS}_{2}$ nanocomposites incorporating a $\beta-\mathrm{NA}$ based on amide derivatives ( $\mathrm{N}, \mathrm{N}^{\prime}-$ dicyclohexyl-2,6-naphthalene, $\mathrm{NJ}$ ) was reported recently and the effect of the $\alpha$ (nano)/ $\beta$ (micro) concentration on the dynamic and isothermal crystallization behavior of iPP was investigated [244,326]. Fig. 35a shows $T_{c}$ values at different cooling rates determined from the DSC cooling curves for iPP nucleated with dual $\alpha\left(\right.$ IF-WS $\left._{2}\right) / \beta(\mathrm{NJ})$ and individual $\alpha$ or $\beta$ NA systems. It was observed that the nucleation effect of 0.1 wt.\% of $\mathrm{NJ}$ was more important than that of 1 wt.\% IF-WS $2\left(T_{\mathrm{C}, \mathrm{PP}}=106.9^{\circ} \mathrm{C}, T_{\mathrm{C}, 1 \mathrm{wt} . \% \text { IF-WS2 }}=119.9^{\circ} \mathrm{C}\right.$ and $T_{\mathrm{C}, 0.1 \mathrm{wt} . \% \mathrm{NJ}}=125.9^{\circ} \mathrm{C}$ at a cooling rate of $10^{\circ} \mathrm{C} / \mathrm{min}$ ) and that for iPP nucleated by dual NA system, the value of $T_{\mathrm{C}}$ for iPP increased and tended to stabilize at concentrations greater or equal to $0.25 \% \mathrm{NJ}$. The same behavior was observed in the NE, which was higher for iPP nucleated with $\alpha\left(\right.$ IF-WS $\left._{2}\right) / \beta(\mathrm{NJ})$ than for iPP $/ \alpha($ IF-WS 2$)$ but still lower than that of iPP/NJ, Fig. 35b. WAXS experiments confirmed that IF-WS $S_{2}$ only induced $\alpha$-crystals while $\mathrm{NJ}$ and the dual $\alpha($ IF-WS 2$) / \beta(\mathrm{NJ})$ induced the $\beta$-form of iPP with a strong dependence on cooling rate and, in the latter case, on the relative content of $\alpha$ and $\beta$ NAs. These studies concluded that the nucleating behavior of the dual additive system could be explained by competitive nucleation [244]. The IF$\mathrm{WS}_{2 /} \mathrm{NJ}$ composition ratio and the cooling rate were very efficient in controlling the content of $\beta$-crystals. The $\beta$ NA induced a progressive inhibition of the $\alpha$-nucleating effect of IF-WS 2 on the matrix even at low weight ratios. Only a very low concentration of $\mathrm{NJ}(0.05 \mathrm{wt} . \%)$ was needed to obtain a fraction of the crystalline $\beta$-phase of $54-67 \%$, and limiting values of $72-86 \%$ fraction of $\beta$-phase were achieved at 0.1 wt.\% NJ, Fig. $35 \mathrm{c}$ and d.
In order to gain insight on the polymorphic transformations, the melting behavior of the different nucleated iPP systems was also studied. It was observed that cooling rate and additive composition had a strong effect on the DSC peaks related to the $\beta \beta^{\prime}$ and $\alpha_{1} \alpha_{2}$ transitions. Moreover, the $\beta-\alpha$ transformation was reduced with slower cooling rate [244]. The same strong dependency on both the NA composition balance and the crystallization temperature was observed for the isothermal crystallization and subsequent melting behavior of iPP nucleated by dual $\alpha\left(\mathrm{IF}_{-} \mathrm{WS}_{2}\right) / \beta(\mathrm{NJ})$ additive systems [326]. This work demonstrated that it was possible to obtain any $\alpha$-phase to $\beta$-phase content ratio by controlling the composition of IF-WS 2 and the $\beta$-NA under appropriate isothermal crystallization conditions. The isothermal crystallization results showed that the largest increment in the crystallization rate of iPP was obtained by the addition of the individual $\mathrm{NJ}$, but the dual system (IF-WS $2 / \mathrm{NJ}$ ) exhibited a more effective nucleating effect than IF-WS 2 . Fig. 36 shows a comparative analysis of the melting curves of iPP nucleated by individual and dual NAs isothermally crystallized and the evolution of the corresponding crystallinities of the different polymorphic forms. Significant changes were observed in temperature and shape of the DSC endotherms for dual $\alpha\left(\mathrm{IF}-\mathrm{WS} \mathrm{S}_{2}\right) / \beta(\mathrm{NJ})$ systems as a function of $\mathrm{NJ}$ content. This behavior was explained due to the complex growth competition phenomenon between the $\alpha$ and $\beta$-phases. At $T_{C}>T(\beta-\alpha)$ the melting curves contained only the $\alpha_{1}$ and $\alpha_{2}$-modifications. The evolution of the crystallinity values of the $\alpha$ - and $\beta$-crystals as well as the relative content of $\beta$-crystals with $T_{c}$, Fig. 36b, were in agreement with this competitive growth between the $\alpha$ and $\beta$ modifications [326].

The effect on the crystallization behavior of iPP of the nucleating duality imposed by mixed nucleating agent systems including other nanoparticles or nanofillers has also been recently reported $[127,198,310,327-330]$. Belfiore et al. [127] demonstrated that the nonisothermal crystallization temperature of iPP increased $14^{\circ} \mathrm{C}$ with respect to pristine iPP when a NA based on an aliphatic triamine $\left(\mathrm{OrganoCH}_{3}\right)$ was efficiently distributed within the polymeric matrix by coating the NA onto zinc oxide nanoparticles. The enhancement in $T_{C}$ was attributed to the efficiency of the NA together with an increase in SSA provided by the $\mathrm{ZnO}$ nanoparticles and an effective distribution of the NA via zinc oxide. While the crystal structure of the $\alpha$-form of iPP was unaffected by zinc oxide, the Organo $\mathrm{CH}_{3}$ induced the formation of $\beta$-iPP crystals. PP-based nanocomposites with nanoclay and nanosized $\mathrm{CaCO}_{3}$ [198] showed a crystallization behavior dominated by the partially exfoliated nanoclay that had a huge SSA and the nucleation effect of $\mathrm{CaCO}_{3}$ was inhibited with no $\beta$-phase formed [198]. This effect resulted in nanocomposites with higher modulus and yield stress but with no significant increase in impact strength with respect to the PP/nanoclay composites. Prachun et al. [327] studied the nonisothermal crystallization behavior of $\mathrm{PP} / \mathrm{PP}-\mathrm{g}-$ $\mathrm{MAH}$ /organo montmorillonite (OMMT) nanocomposites modified with an aryl amide $\beta$-nucleator to promote the formation of the $\beta$-phase during melt crystallization. It was found that the $\beta$-crystal modification affected the thermal 

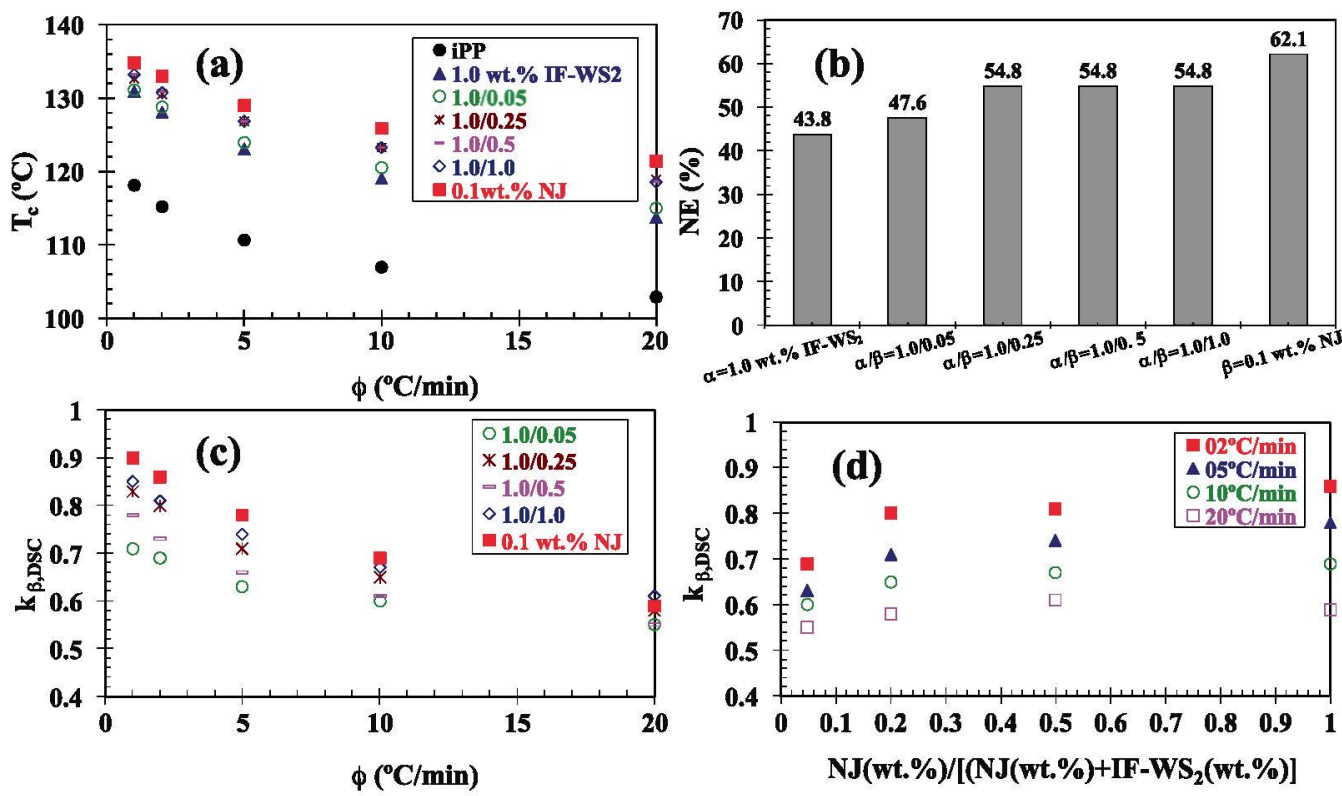

Fig. 35. (a) Variation of the crystallization peak temperature $\left(T_{\mathrm{p}}\right.$ ) with the cooling rate, (b) nucleating efficiency (NE), (c) Variation of the relative content of $\beta$-crystals $\left(k_{\beta}\right.$, DSC) with the cooling rate and (d) the relative NJ content, for iPP nucleated with $\alpha($ IF-WS 2$) / \beta(N J) N A$ and individual $\beta$ NA. Reprinted with permission [244]. Copyright 2011, American Chemical Society.

and mechanical properties of PP/PP-g-MAH/OMMT nanocomposites. $T_{\mathrm{C}}$ of the PP/PP-g-MAH/OMMT nanocomposite was observed to be slightly higher by $3^{\circ} \mathrm{C}$ than that of neat iPP, whereas the $\beta$-nucleation increased $T_{C}$ of the PP/PP-g-MAH/OMMT/aryl amide by $15^{\circ} \mathrm{C}$, to $128^{\circ} \mathrm{C}$. Other positive effects of the $\beta$-nucleation of these nanocomposites were a slight reduction in density and an improvement in the very low elongation at break by $207 \%$ at a $\beta$-form level of $92 \%$. Zhang et al. [328] investigated the combined effect of a $\beta$-NA based on rare earth (WGB) and multi-walled carbon nanotubes (MWCNTs) on the polymorphic composition and morphology of iPP. The mixed nucleating system imposed a dual nucleating effect inducing a competitive growth between $\alpha$-crystals nucleated by MWCNTs and $\beta$-crystals nucleated by WGB. The polymorphic composition could be adjusted by the variation of filler/ $\beta$-NA proportion and it was observed that the $\beta$-nucleation ability of WGB did not decrease monotonically with increasing MWCNT content, but an optimum filler proportion attained the highest $\beta$ content. These mixed nucleating systems present a similar behavior to that previously described for the iPP/IF-WS 2 nanocomposites incorporating the $\beta$-NA (NJ) with competitive nucleation between the fillers. However, in general the increments observed in the crystallization temperature of iPP in IF-based nanocomposites with the addition of NAs are higher than those achieved with other nanofillers.

A different approach was to chemically support the $\beta$ NA calcium pimelate onto the surface of MWCNTs [329] or in nano- $\mathrm{CaCO}_{3}$ [327]. The MWCNT supported $\beta-\mathrm{NA}$ could successfully enhance the $\beta$-nucleating ability of the agent and could induce a higher content of the $\beta$ phase of iPP, which significantly improved the impact toughness without deteriorating the strength and stiffness of the iPP nanocomposites [329].
The use of NAs directly mixed or chemically supported on nanomaterials provides an innovative method for increasing the efficiency of these polymer additives with a reduction in their content, and emerges as a tool for tuning the properties of the nanocomposites by controlling the parameters that affect the dual nucleation behavior. The promising results obtained with the use of IFs in mixed nucleating systems open a new pathway for further developments in this direction for thermoplastic IF-PNCs.

\subsection{IF-PNCs containing carbon nanotubes. The case of high performance thermoplastics}

CNTs are one of the most attractive nanomaterials and a considerable area of research, due to their unique structure and exceptional thermal, electrical, magnetic and mechanical properties [331,332]. These outstanding properties make them ideal candidates for many potential applications such as molecular electronics, energy conversion and storage, sensors, biosensors and biomedicine and advanced composite materials [10,333-337]. During the last decade a great effort has been directed towards the efficient incorporation of CNTs into polymer matrices for structural and functional applications [10,337-339]. However, the main challenge still remains in transferring the properties of these nanomaterials to the polymer matrix successfully. Difficulties associated with dispersion of entangled nanotubes during processing and weak nanofiller-matrix interfacial interactions are the two main factors that limit the full potential of polymer/CNT composites. CNTs usually form bundles due to van der Waals interactions and are extremely difficult to disperse in a polymer matrix. Moreover, the carbon atoms on CNT walls are chemically stable because of the aromatic nature of the bond and interact with the polymer matrix mainly through van der Waals 

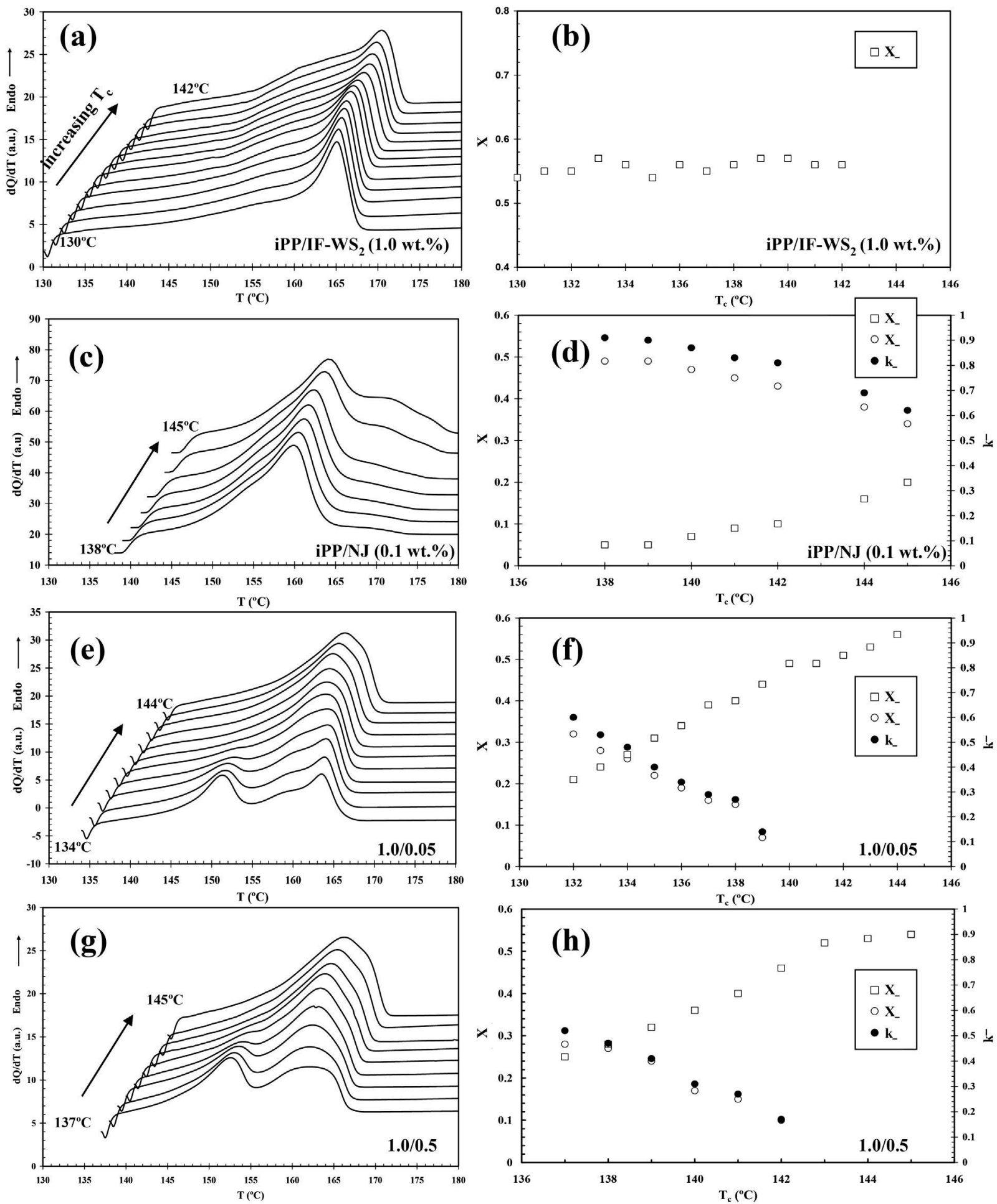

Fig. 36. Melting behavior of iPP nucleated with dual $\alpha($ IF-WS $) / \beta(\mathrm{NJ})$ NAs and individual $\alpha$ or $\beta$ NAs obtained at a heating rate of $5^{\circ} \mathrm{C} / \mathrm{min}$ after isothermal crystallization. Left panel shows the evolution of DSC melting thermograms of iPP systems with $T_{\mathrm{C}}$ and right panel illustrates the corresponding crystallinity values of the $\alpha$-form, $X_{\alpha}$, and $\beta$-form, $X_{B}$, and the $\beta$-form content $\left(k_{\beta}\right)$.

Reprinted with permission [326]. Copyright 2011, American Chemical Society. 
interactions that generally do not provide efficient load transfer across the CNT/matrix interface. Several recent critical reviews discuss different approaches developed to overcome these two problems [339-342]. Strategies based on mechanical dispersion with ultrasonication, ball milling, ultrasound assisted extrusion, high shear mixing, latex technology, in situ polymerization, coagulation spinning or by using external magnetic/electric fields are only some of the methods used to obtain a proper dispersion of CNT in polymers $[10,340-342]$. Non-covalent functionalization of nanotubes using surfactants, biomacromolecules or wrapping with polymers as well as covalent functionalization including the use of click chemistry and hyperbranched polymers are techniques used to modify the surface properties of CNTs and to achieve good CNT dispersion and interactions with the matrix in order to use them as effective reinforcements in PNCs [339-341]. The strategies employed for CNT dispersion and functionalization have an important influence on the mechanical, thermal, and electrical performance of the polymer/CNT composites.

An innovative route for the design of new multifunctional polymer/CNT nanocomposites has been the combination of CNTs with inorganic nanoparticles in order to obtain hybrid nanocomposites with synergetic behavior and, in some cases to overcome some of the drawbacks of the incorporation of CNTs in polymers, with potential applications in electronic or nanoelectronic devices, gas sensing and catalysis, among others. Very recent efforts have focused on the preparation of polymer/CNT nanocomposites which incorporate inorganic nanofillers to enhance specific properties such as barium titanate (BT) nanoparticles to improve the dielectric properties [343], iron oxide nanoparticles in the crystalline form of magnetite [344] for magnetic properties, titanium dioxide nanoparticles for photocatalytic properties [345], metals ( $\mathrm{Au}$ and $\mathrm{Ag}$ ) nanoparticles for electrical or antimicrobial properties [346-349], silica nanoparticles [350] and nanoclay [351] for mechanical properties, and IFs for thermal, mechanical and tribological properties [238,239,266,387,311].

New hybrid nanocomposites of PEEK and PPS incorporating $\mathrm{IF}^{-\mathrm{WS}_{2}}$ and single-walled carbon nanotubes (SWCNTs) prepared by melt blending have been recently reported $[238,239,266,287,311]$. To improve the dispersion of SWCNTs within the PPS matrix, the nanotubes were wrapped in polyetherimide (PEI) while in the case of PEEK they were incorporated without modification. It was observed that the IF-WS 2 nanoparticles played an effective lubricant role improving the dispersion of SWCNTs in both PEEK/SWCNT and PPS/SWCNT-PEI nanocomposites [238,311]. SEM micrographs of PEEK/SWCNT/IF$\mathrm{WS}_{2}$ hybrid nanocomposites prepared by two different processing strategies are given in Fig. 37. The simultaneous mixing of fillers resulted in significant debundling of the SWCNTs, Fig. 37a, but when the fillers were added in two steps the SWCNTs were not uniformly dispersed and some aggregates could be clearly observed, Fig. 37b. The effective dispersion of SWCNTs achieved by the addition of IF-WS 2 nanoparticles resulted in improved properties of the hybrid nanocomposites. The thermal stability increased with respect to that of the polymer matrix but the behavior and dependence on composition varied for PEEK and PPS.
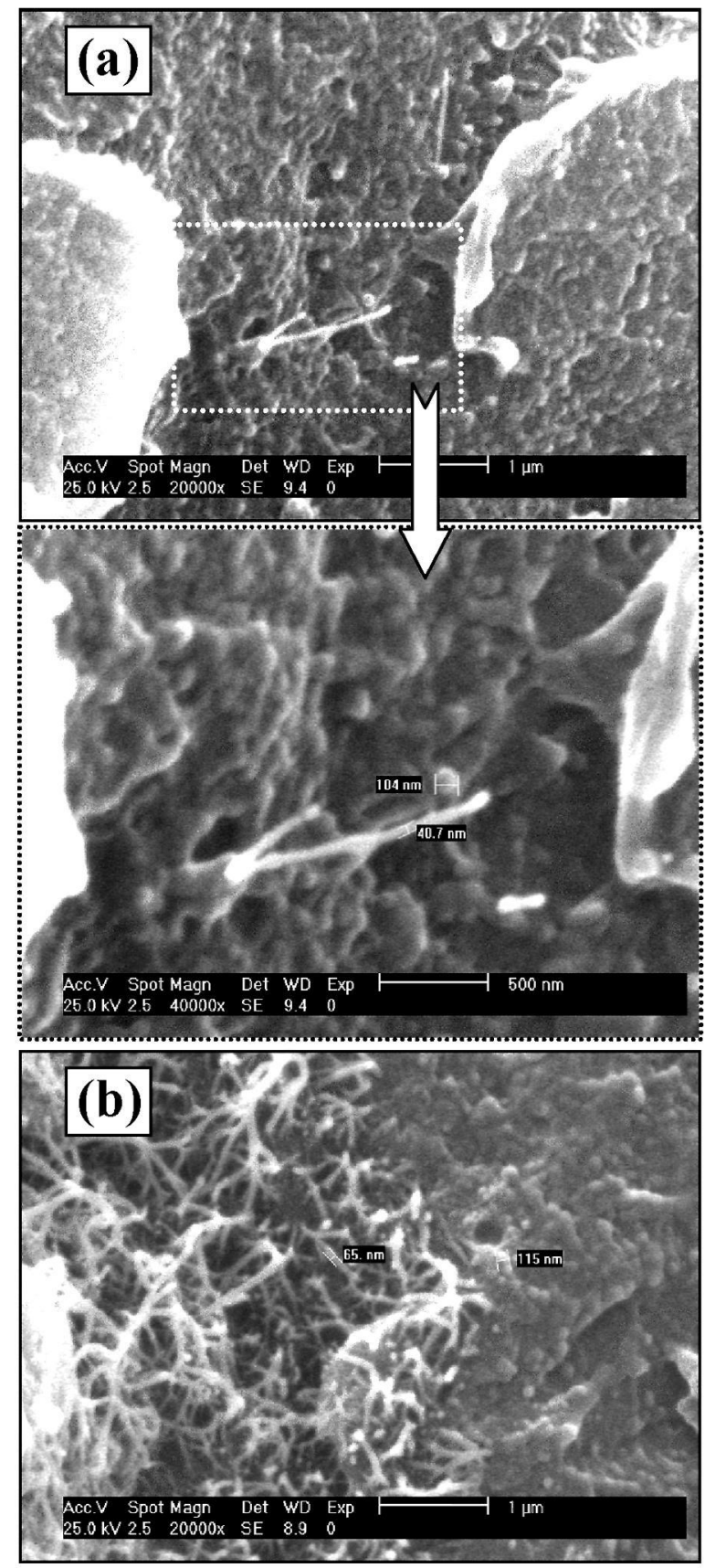

Fig. 37. SEM micrographs of melt-processable PEEK/SWCNT/IF-WS ( $x=y=0.5$ wt.\%) obtained during (a) one and (b) two mixing steps. Reprinted with permission [311]. Copyright 2011, Royal Society of Chemistry.

Fig. 38 compares the difference between the initial degradation temperature of hybrid nanocomposites (PEEK and PPS) and the pure polymers, $\Delta T_{\mathrm{i}}$, as a function of the relative IF-WS ${ }_{2}$ content. For PEEK reinforced by SWCNT/IF-WS 2 the value of $\Delta T_{\mathrm{i}}$ increases and tends to stabilize at concentrations greater or equal to 50\%. In PPS systems there is an optimum ratio of fillers, corresponding to a relative concentration of IF-WS 2 around $25 \%$ that leads to the highest 


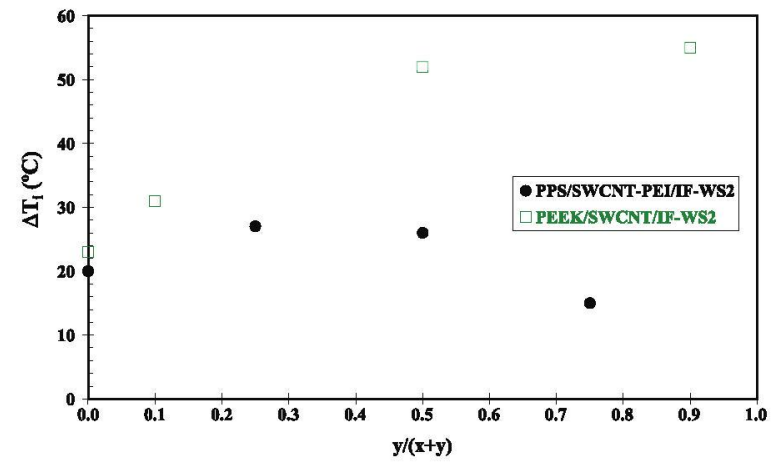

Fig. 38. Comparison of the difference between the $T_{i}$ of hybrid nanocomposites and pure polymer $\left(\Delta T_{i}\right)$ for PPS and PEEK with the relative IF-WS content.

Reprinted with permission [238]. Copyright 2012, Royal Society of Chemistry.
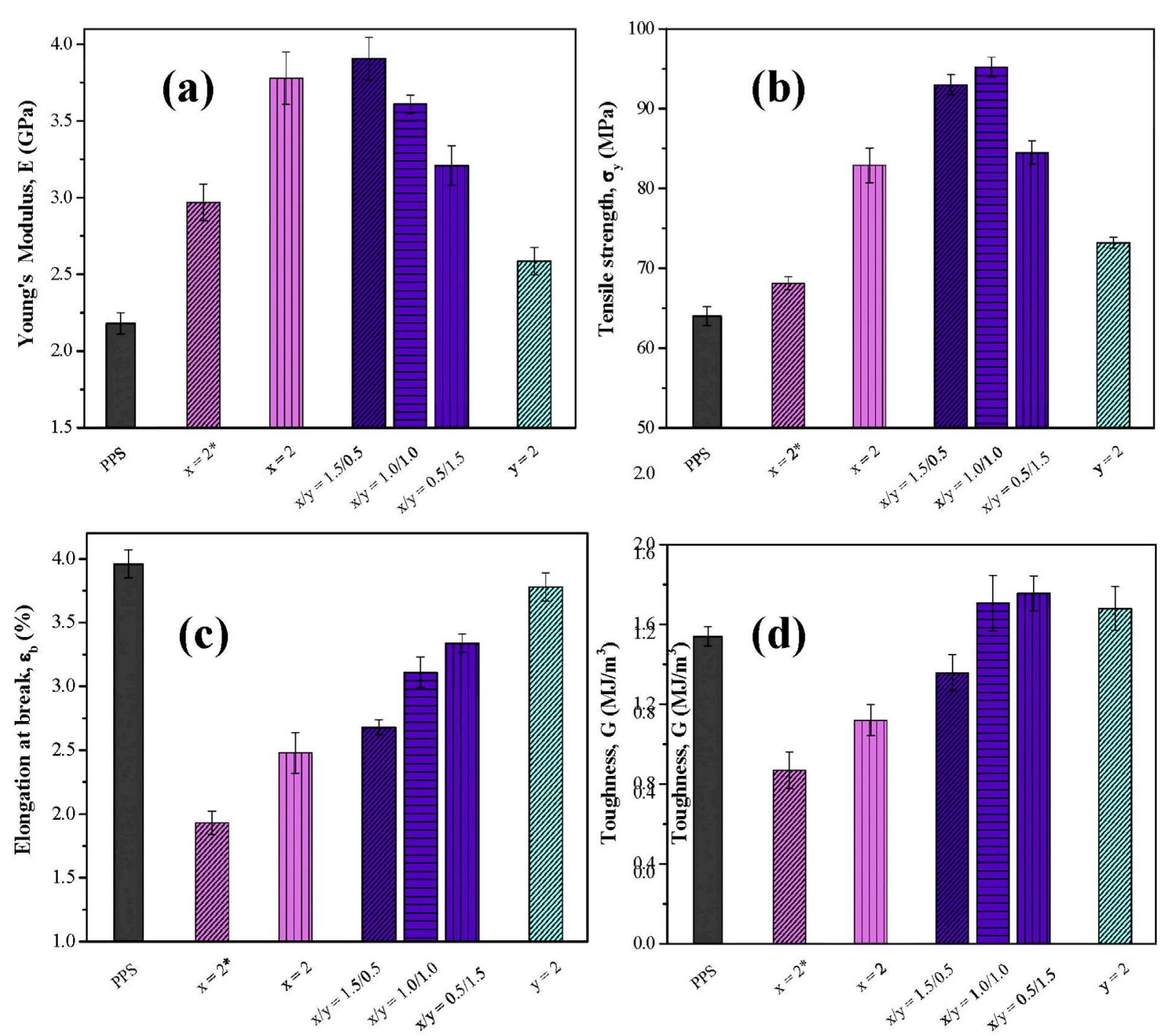

Fig. 39. (a) Young's modulus, (b) tensile strength, (c) elongation at break and (d) toughness for SWCNT/IF-WS 2 /PPS composites. For comparison, data of SWCNT/PPS and IF-WS 2 /PPS composites are also plotted.

Reprinted with permission [266]. Copyright 2012, Elsevier.

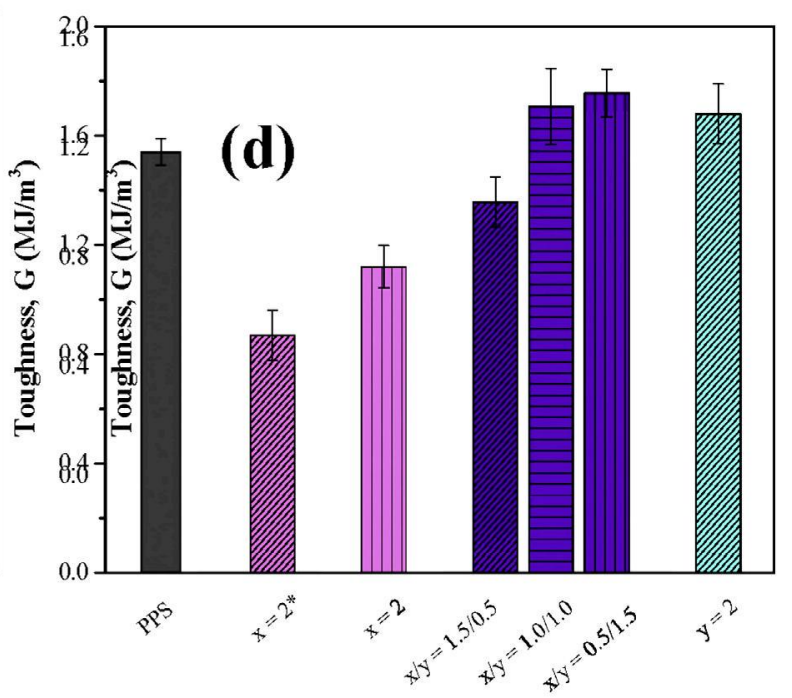

value of thermal stability. The increment of the degradation temperature in the hybrid nanocomposites was attributed to a synergistic stabilization effect induced by the improved filler dispersion where the SWCNTs and IF-WS 2 nanofillers efficiently hinder the diffusion of the degradation products, and the increase in the thermal conductivity observed in the hybrid composites was consistent with the thermal stability results [238,311]. The largest improvement of $130 \%$ in the thermal conductivity of PEEK was obtained by simultaneous addition of equal concentrations of SWCNTs and IF-WS 2 , which was higher than the reported value obtained for PEEK/SWCNT composites incorporating compatibilizer agents such as polysulfones [352]. In the case of PPS the highest thermal conductivity value, superior by almost $150 \%$ to that of neat PPS, was obtained for the hybrid nanocomposite with a relative IF-WS 2 content of $25 \%$. These results were considered of significant relevance since the addition of much cheaper IF-WS 2 nanoparticles 


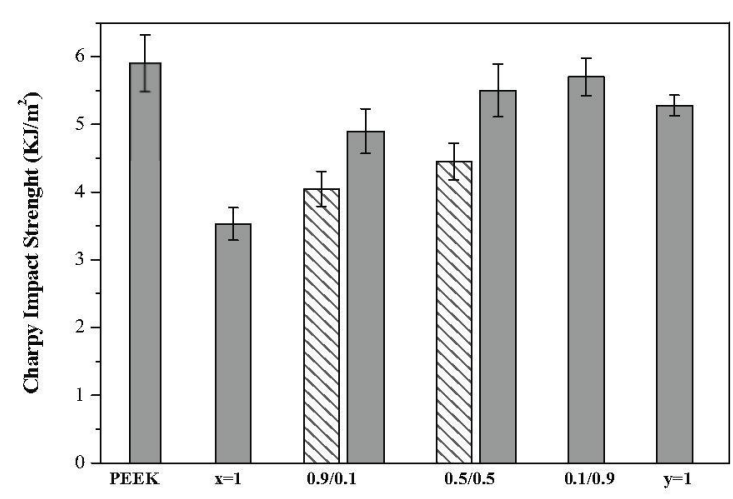

Fig. 40. Charpy notched impact strength measurements at $23^{\circ} \mathrm{C}$ for PEEK and the different nanocomposites. The dashed bars correspond to composites manufactured in two stages.

Reprinted with permission [311]. Copyright 2011, Royal Society of Chemistry.

in comparison to CNTs produced a remarkable enhancement in the thermal conductivity of both PPS and PEEK. Furthermore, this enhancement could allow a reduction in cycle times during processing improving the production efficiency of these high performance polymers. However, all hybrid nanocomposites showed a lower overall crystallization rate than the neat matrix [238,311]. As has been discussed in Section 4.3, while IF-WS 2 can act as a nucleating agent in PPS [226] it does not have a nucleating effect in PEEK [57]. Additionally, SWCNTs do not exert a nucleating effect neither on PPS [238] nor PEEK [242] unlike that observed in other polymers [353]. However, it was observed that in PPS/SWCNT-PEI/IF-WS 2 nanocomposites the nucleation capacity of the IF-WS $\mathrm{S}_{2}$ nanoparticles was cancelled out. Considering the values of the crystallization activation energy, the hybrid nanocomposites had higher values than those of the pure polymers indicating that the disadvantage of SWCNTs with respect to the difficulty of transport of polymer segments to the growing surface was not outweighed by the improved CNT dispersion and nucleation ability of the IF-WS 2 . The crystallinity of PEEK and PPS was also lower in the hybrid composites. However, the crystalline structure of PEEK and PPS was unchanged with the addition of SWCNTs and IF-WS 2 . Regarding the mechanical properties, significant enhancements in stiffness, strength and toughness were observed in PEEK and PPS hybrid composites by the addition of both nanofillers $[239,266]$.

Fig. 39 shows the evolution of the Young modulus $(E)$, tensile strength $\left(\sigma_{\mathrm{y}}\right)$, elongation at break $\left(\varepsilon_{\mathrm{b}}\right)$ and toughness $(G)$ for PPS/SWCNT/IF-WS 2 composites as a function of the nanofiller content. The hybrid composites had higher modulus than the binary composites due to a synergistic reinforcement effect of SWCNTs and IF-WS 2 . Similar results were observed for the tensile strength. Nevertheless, the ductility and toughness slightly decreased in the hybrids although they were above the values of the binary SWCNTs composites as observed in the Charpy notched impact strength measurements showed in Fig. 40 for PEEK/SWCNT/IF-WS ${ }_{2}$ composites. DMA experiments demonstrated a strong increase in the storage modulus and $T_{g}$ of the matrix by the addition of both nanofillers

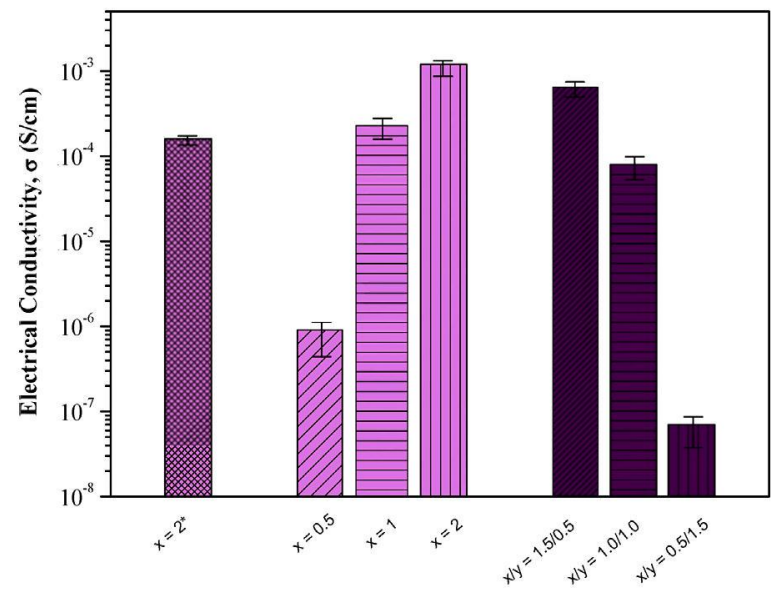

Fig. 41. Comparison of the room temperature electrical conductivity of SWCNT/PPS and SWCNT/IF-WS $/$ /PPS composites. Note that pure PPS and IF-WS 2 /PPS composites are electrically insulating $\left(\sigma<10^{-8} \mathrm{~S} / \mathrm{cm}\right)$, hence are not included in the plot.

Reprinted with permission [266]. Copyright 2012, Elsevier.

$[239,266]$. This is consistent with the changes observed in the absorption peaks of the ATR-FTIR spectra of the PEEK/SWCNT/IF-WS 2 nanocomposites in comparison to the neat polymer, which suggested the presence of potential interations between the nanofillers and the polymer matrix [239]. For example, the formation of hydrogen bonds between the carbonyl group of PEEK and the carboxylic groups of the SWCNTs, evidenced by the broadening and diminution in the intensity of the carbonyl band at $1651 \mathrm{~cm}^{-1}$, as well as a slight shift to lower frequencies, and modifications in several other absorption bands of the nanocomposites was thought to also be related to the existence of SWCNT-matrix interactions [58,239]. The electrical conductivity of PEEK and PPS increased dramatically by the incorporation of SWCNTs, and the replacement of a small amount of SWCNTs with IF-WS 2 had an almost negligible effect on the electrical conductivity behavior of the hybrid nanocomposites, as shown in Fig. 41 for PPS/SWCNT/IF-WS 2 . Finally, composites with both nanofillers exhibited improved wear behavior that was attributed to the outstanding tribological properties of the IF-WS 2 nanoparticles and a synergistic effect between nanofillers on the enhancement of the sliding wear resistance [287]. In Fig. 42 the COF and wear rate for PEEK/SWCNT/IF-WS 2 composites are compared with PEEK/SWCNT composites. A significant decrease in both parameters was observed in the hybrids compared to the samples reinforced solely with SWCNTs. It was concluded that the hybrids incorporating low IF-WS 2 content had superior overall performance than composites reinforced solely with SWCNTs and their properties could be tailored by modifying the SWCNT/IF-WS 2 content.

Synergistic effect was also reported for polymer/CNT composites with the addition of different inorganic nanofillers, to both thermoplastic and thermosetting resins. Hsieh et al. reported the effect of silica nanoparticles and MWCNTs on the mechanical properties of an epoxy resin [350]. The addition of silica nanoparticles alone led to a significant increase in the fracture energy for the 

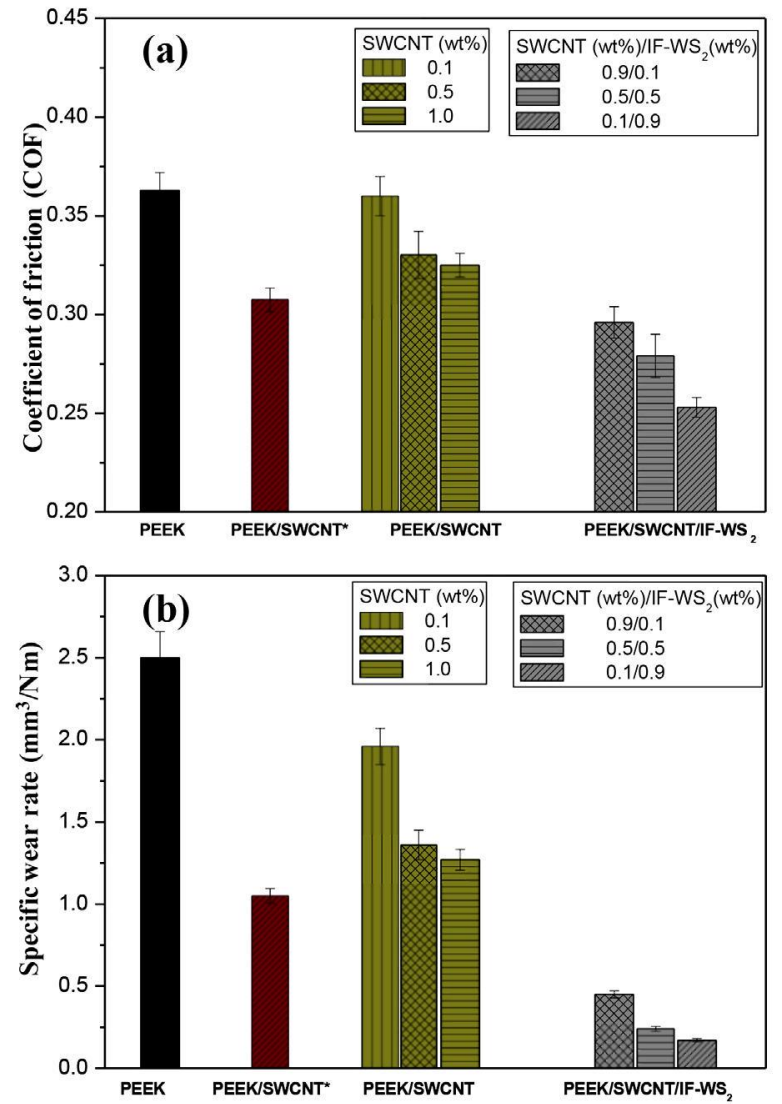

Fig. 42. Comparison of the coefficient of friction (a) and wear rate (b) for PEEK and the different nanocomposites.

Reprinted with permission [287]. Copyright 2012, American Chemical Society.

nanocomposites, up to $36 \%$ for 6 wt.\% of silica nanoparticles. The addition of a low content of MWCNTs to form hybrid nanocomposites led to further enhancement in toughness explained by a mechanism which involved pull out of the CNTs and void initiation and growth, which involved the plastic deformation of the epoxy associated with the presence of the silica nanoparticles and the MWCNTs [350]. Highly reinforced hybrid epoxy nanocomposites were also prepared by incorporation of synthetic inorganic alpha-zirconium phosphate, $\mathrm{ZrP}$ nanoplatelets and CNTs [351]. The inorganic nanoplatelets were used to disperse the CNTs in aqueous suspensions due to the strong electrostatic affinity between the fillers, which were transferred into organic solvents and, then into the epoxy matrix. Hybrid epoxy nanocomposites containing low concentrations of both CNTs and nanoplatelets showed exceptionally high mechanical strength and modulus without reduction in elongation at break [351]. Yu et al. [343] reported the preparation by melt processing of hybrid PP nanocomposites with MWCNTs as the electrically conductive component, and spherical BT nanoparticles as the ferroelectric component. While more than 3 wt.\% of MWCNT was required to make PP electrically conductive, only $2 \mathrm{wt} . \%$ of MWCNTs were sufficient to make $\mathrm{PP} / \mathrm{MWCNT/BT}$ ternary nanocomposites electrically conductive using surface modified MWCNTs and BT. The enhancement in conductivity and decrease in the percolation threshold was attributed to the reduced viscosity due to the surface modification and volume exclusion effect of BT nanoparticles. Moreover, the incorporation of MWCNTs greatly increased the dielectric permittivity of PP/BT nanocomposites [343]. Synergetic effects between SWCNTs and Ag nanoparticles in PCL were reported by Fortunati et al. [348] to affect the morphological, electrical, dielectrical, mechanical and biological properties of the hybrid nanocomposites. A remarkable improvement in electrical conductivity was due to the formation of conductive pathways facilitated by the silver nanoparticles that acted as conductive bridges among the nanotube bundles enabling electron transfer. The suitability of these conductive composites as a potential support for primary human bone marrow-mesenchymal stem cells was also demonstrated.

Simultaneous addition of CNTs and inorganic nanoparticles as reinforcements in polymer matrices result in advanced multifunctional hybrid nanocomposites with a remarkable improvement in properties due to the synergistic effect of the fillers. One of the main advantages has been the possibility to overcome one of the drawbacks of the incorporation of CNT in polymers, i.e. to obtain a homogeneous dispersion of the carbon nanofillers. In this context, the use of IF-WS 2 , a low cost and environmentally friendly reinforcing filler, has shown to be a very efficient strategy to improve the performance and processability of polymer/CNT nanocomposites. Considering the excellent results obtained for high performance polymers further developments related with other thermoplastic polymers can be envisaged.

\subsection{IF-PNCs with carbon fibres}

Carbon fiber-reinforced polymer composites (CFRPs) are widely used in many structural applications including aerospace, automobile and civil and marine structures due to their high modulus and strength combined with low weight $[354,355]$. While these materials have excellent inplane mechanical properties, their interlaminar properties are limited by weak fiber matrix interfacial bonding. Different strategies have been used during the last decades to improve the delamination resistance but usually at the cost of in-plane mechanical properties [356-359]. Recently there is an increasing interest in the development of hierarchical (or multiscale) composites, in which nanoscale reinforcements are combined with traditional microscale reinforcing fibers [356-365]. Besides the improvement in mechanical performance these nanofillers can add other functionalities. The introduction of CNTs into CFRPs have demonstrated to significantly improved the composites performance [359-361], and the effects on the properties of the hierarchical composites of dispersing CNTs in the matrix or attaching CNTs on the fiber surface have been recently reviewed $[359,360]$. Other nanofillers have been incorporated in CFRPs such as layered silicates [365], metal oxide nanoparticles [362], POSS [364], and IF-WS 2 nanoparticles [363], and the mechanical enhancement observed in these multiscale composites has been attributed to the synergistic effect of CFs and inorganic nanoparticles. 

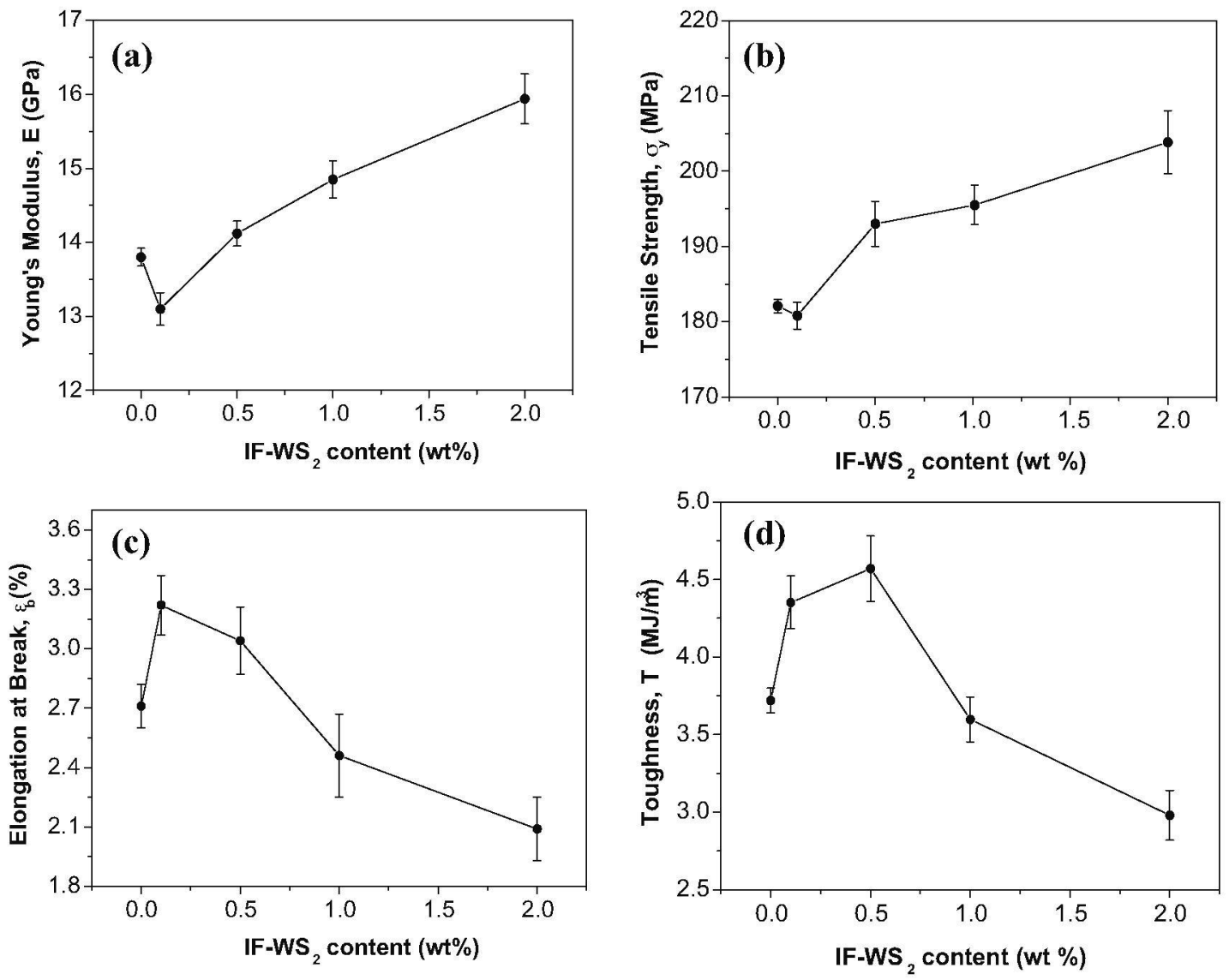

Fig. 43. (a) Young's modulus, (b) tensile strength, (c) elongation at break and (d) toughness obtained from the tensile tests of PPS/IF-WS $/$ /CF laminates as a function of nanoparticle loading.

Reprinted with permission [363]. Copyright 2012, Elsevier.

Hybrid composites composed of alternating layers of PPS/IF-WS 2 films and carbon fabrics were prepared by melt-blending and hot-press processing [363]. SEM analysis of these materials demonstrated efficient nanoparticle dispersion within the matrix without the need for surfactants or compatibilizing agents, and a significant enhancement in thermal stability was found with increasing IF-WS 2 loading due to the barrier effect of the nanoparticles that effectively hindered the transport of volatile decomposed products from the bulk of the polymer to the gas phase, decelerating the decomposition process. Regarding the influence of the nanoparticles in the crystallization of PPS in the laminates, a similar effect from inhibition to promotion with increasing IF$\mathrm{WS}_{2}$ content was observed as that previously described for PPS/IF-WS 2 binary composites $[168,226]$. However, while in the binary composites the addition of $0.5 \mathrm{wt} . \%$ IF-WS 2 increased the crystallization temperature, in the hybrid laminates nanoparticle content equal or higher than $1 \mathrm{wt} . \%$ was required to promote polymer crystallization. These changes were attributed to the presence of the CFs that inhibited the nucleating effect of IF-WS ${ }_{2}$. Changes in $T_{g}$ were also observed in the DSC thermograms and more clearly detected by DMA, with a downshift in $T_{\mathrm{g}}$ in the hybrids with respect to the reference laminate at low nanoparticle content and an increase for those with IF$\mathrm{WS}_{2}$ loadings $\geq 1 \mathrm{wt} . \%$. The differences in $T_{\mathrm{g}}$ arose from the changes in crystallinity observed by DSC in the hybrid laminates as a function of the IF-WS ${ }_{2}$ content. The mechanical properties also reflected the differences observed in the crystallization behavior of the hybrid nanocomposites depending on nanoparticle concentration [363]. The addition of very small amounts of IF-WS 2 slightly decreased the storage, tensile and flexural moduli, but they all increased remarkably at higher concentrations, in particular the outof-plane mechanical properties. The tensile properties of the PPS/IF-WS $/$ CF laminates obtained from the room temperature stress-strain curves are shown in Fig. 43. An increment of $16 \%$ for the Young's modulus and $12 \%$ for the tensile strength were observed for the hybrid composites with the highest nanoparticle concentration of $2 \mathrm{wt} . \%$. The results obtained from the flexural tests and from the Charpy impact tests are shown in Fig. 44. The flexural modulus of the hybrids increased up to 17 and $24 \%$ at 1 and $2 \mathrm{wt} \%$ IF-WS 2 , respectively, and the flexural strength to 14 and $17 \%$ for the same loadings, Fig. $44 a$. An increase in impact strength was observed for low nanoparticle content, but increased loading led to a decrease in this parameter in agreement with the crystallinity changes of the matrix, Fig. 44b and an optimum balance between 

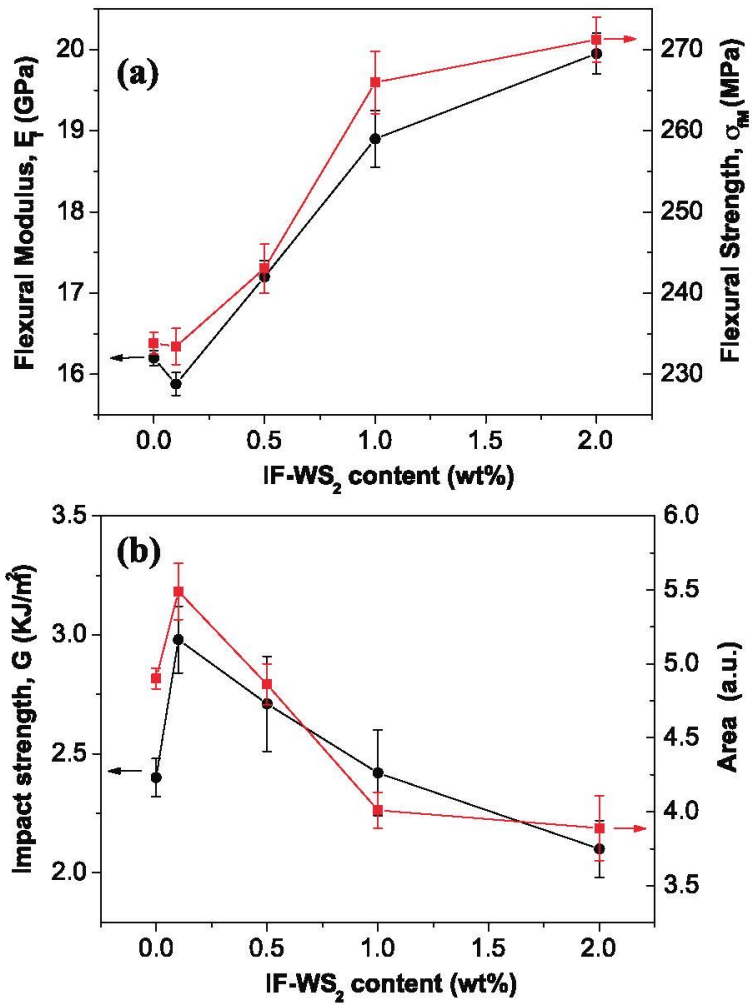

Fig. 44. (a) Effect of IF-WS 2 concentration on the room temperature flex ural modulus and strength of PPS/IF-WS $/$ /CF laminates, (b) evolution of Charpy notched impact strength at $23^{\circ} \mathrm{C}$ and area under the tan $\delta$ peak obtained from DMA measurements as a function of nanoparticle content for PPS/IF-WS $/$ /CF laminates.

Adapted with permission [363]. Copyright 2012, Elsevier.

stiffness and toughness was achieved at $1 \mathrm{wt} . \% \mathrm{IF}-\mathrm{WS}_{2}$. Moreover, the microhardness of the multiscale composites increased steadily with the nanoparticle content whilst the COF and wear rate strongly decreased, as can be observed in Fig. 45. The remarkable increase in wear resistance was attributed to a synergistic effect between the IF-WS 2 and the CFs. The lubricant role and the mechanism for the improved tribological behavior of IF-WS 2 spherical nanoparticles in which the interplay between the rolling/sliding of the nanoparticles play a major role [366] has been previously discussed. In the PPS/IF-WS $2 / C F$ laminates it was found that fiber breakage and debonding were minimized by the presence of the nanoparticles considered as the key factors for enhancing the wear resistance [363].

A similar trend in mechanical performance and especially in the wear behavior has been reported for different multiscale polymer/CF composites incorporating other inorganic nanoparticles. The influence of nano- $\mathrm{ZrO}_{2}$ particles on the mechanical and wear properties of PEEK/CF composites was investigated by Lin et al. [362]. The excellent wear resistance of the $\mathrm{PEEK} / \mathrm{ZrO}_{2} / \mathrm{CF}$ composites was attributed to a synergetic effect between the nanoparticles and $C F$. While the CF carried the majority of load during the sliding process protecting the matrix from severe abrasion, the incorporation of nano- $\mathrm{ZrO}_{2}$ inhibited CF failure reducing the stress concentration on the $\mathrm{CF}$ interface
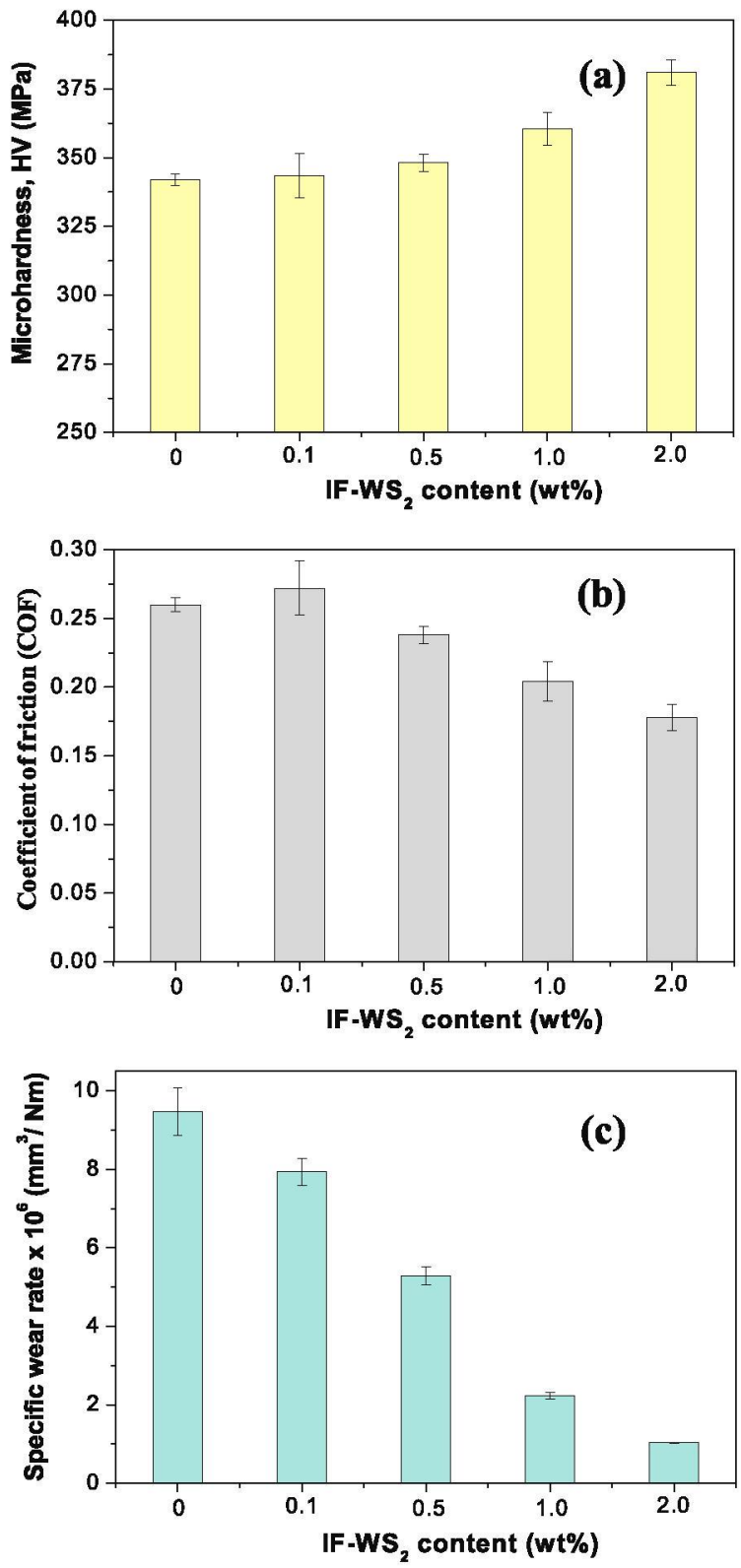

Fig. 45. (a) Vickers microhardness, (b) coefficient of friction and (c) specific wear rate of the laminates prepared as a function of nanoparticle content.

Reprinted with permission [363]. Copyright 2012, Elsevier.

and the shear stress between sliding surfaces. Friedrich et al. [288] reviewed the effect of various fillers on the sliding wear of polymer composites and showed that the combination of short carbon fibers (SCFs) with inorganic nanoparticles (nano- $\mathrm{TiO}_{2}$ ) exerted a clear improvement in friction and wear performance in both thermosetting and thermoplastic composites, which was explained as a topographic smoothening and a possible rolling effect due to the nanoparticles. In particular, the incorporation of nano $\mathrm{TiO}_{2}$ or micro-CaSiO 3 into PEI reinforced with SCFs reduced the wear rate and COF of the PEI composites and this enhancement of the tribological properties 
was attributed to the formation of transfer layers on both sliding surfaces together with the reinforcing effect [367]. Regarding other mechanical properties, the addition of nano- $\mathrm{Al}_{2} \mathrm{O}_{3}$ particles to CFRPs significantly improved the Young's modulus, interlaminar shear strength and thermal contraction of the composites [368]. Moreover, when layered clays were used as nanoparticle fillers in CF-reinforced thermosetting polymer composites the transverse cracking was significantly reduced and the reinforcement effect was completely dependent on nanoparticle concentration and morphology [369].

Incorporation of nanoparticles into CFRPs offers new possibilities to improve performance of these materials, especially the weak properties in the transverse direction, and provide a vehicle to add other functionalities. In these systems the physical and mechanical interactions between the nanofiller and the microscale fiber reinforcement play a very important role. The first results obtained with the addition of IF-WS 2 nanoparticles to CFRPs open a new route to property improvements in multiscale composites in which the excellent tribological properties of these spherical nanoparticles and their uniform dispersion within polymer matrices present clear advantages with respect to other nanofillers.

\section{Future directions and potential applications}

Hollow closed inorganic fullerene-like nanoparticles such as IF-WS 2 are currently synthesized in large amounts by ApNano Materials, Inc. (NanoMaterials, Ltd.) and employed in a wide variety of fields such as aerospace, automotive, naval, defense, medical, energy, electronics, and various other industries. As was discussed in Section 2, in contrast to organic fullerenes IFs are easier and much cheaper to produce, chemically more stable and consequently less flammable. One of their most interesting properties is an extremely high shock absorbing ability. Shock absorbing materials are commonly used in impact resistant applications such as ballistic protection, personal body armor, bullet-proof vests, vehicle armor, shields, helmets, and protective enclosures [370]. On the other hand, inorganic lubricant additives based on IF-WS ${ }_{2}$ nanoparticles are commercially available as an oil concentrate or paste for improving the performance of lubricating oils, greases and dry lubricants used in crankcases, gears, transfer cases, bicycles, saws, conveyors, etc. They are also provided in powder form, and are widely used for solid lubricants in pumps and blowers as anti-friction modifiers to fabricate coatings and mainly for the preparation of high performance polymer nanocomposites.

Medical technologies also benefit from the excellent tribological and mechanical behavior of IF nanoparticles and their future prospects have been discussed in two recent reviews [26,371]. Nevertheless, the research in this field is quite new, and is limited to very few research groups. Studies on the toxicity of IF-WS 2 are limited and the number of tested taxa are few [371]. These nanoparticles are currently being investigated for new medical applications $[372,373]$ such as biomarkers, vectors for drug delivery or for hypothermia treatment, imaging contrast agents, and for a variety of other therapeutic practices. They also have a wide number of potential dental applications including, amongst others, coated orthodontic wires that are inserted onto brackets, endodontic files and dental creams [372,373].

It is important to highlight that these nanometric-sized particles can also be combined with organic materials, usually polymeric in nature, in order to obtain composites with improved properties. Recent advances in the synthesis and scale-up of IF-WS $\mathrm{W}_{2}$ have brought this type of nanomaterials closer to the market, especially in the case of the preparation of polymer nanocomposites. By combining the merits of organic and inorganic materials into novel polymer/IFs, different types of nanocomposite materials and hybrids have been successfully developed ranging from high-performance to commodity thermoplastic-based systems. The incorporation of IF-WS 2 into thermoplastic polymers offers the opportunity to develop a large amount of novel materials with high potential for new applications, making them a vibrant research area for the next years. For optimal control of the properties of these new materials, it is highly important to tailor the fabrication process from the viewpoint of the final product. Obviously, the improvement and application of these nanocomposites depend on how effectively we optimize and scale-up their fabrication method. Due to their lubricant character, IF-WS 2 reduce the viscosity of the thermoplastic/nanofiller mixture when it is exposed to high shear forces and heat during the melt-blending process, promoting a very uniform dispersion within the matrix without the need for surfactants or modifiers [287]. However, for specific applications, the nanoparticles should be surface functionalized in order to confer more selectivity, specificity and reactivity with the polymer chains $[374,375]$. On the other hand, doping of these nanoparticles can also lead to a marked increase in their conductivity, and reduce agglomeration, friction and wear to levels approaching superlubricity [376]. Significant progress in the understanding of these nanocomposites within the last few years is promising, especially for the field of biocompatible and/or biodegradable polymeric composites for medical applications, and successful development is feasible, due to the intrinsic properties of the IF-WS ${ }_{2}$. Morevoer, driven by a growing consciousness for the environment and new standards, there is also a great interest to develop sustainable and environmentally friendly systems with enhanced performance. In particular, the modification of biodegradable biopolymers to make them more competitive with petroleum based polymers in terms of performance and cost is an important objective. A simple and efficient method to improve the properties of biopolymers and greatly enhance their commercial potential is the incorporation of fullerene-like nanoreinforcements.

\section{Conclusion}

For many current applications of thermoplastics, additives and fillers need to be incorporated into the polymers in order to achieve optimized performance requirements, cost and processing. The use of nanoscale fillers benefits from the advantages of the nanometre-sized particles in comparison with macro- or microscopic fillers. Inorganic 
fullerene-like (IF) nanoparticles are emerging as a novel type of nanomaterial, offering significant advantages over other spherical inorganic nanoparticles for the preparation of advanced polymer nanocomposites. A wide range of new melt-processable polymer/IF formulations has already been developed by dispersing these nanoparticles in molten thermoplastics. By combining both organic and inorganic components the resulting nanocomposites can find broad applications in fields such as defense, automotive, aerospace, naval, electronics and more recently in medical technology. Some of these applications are still at an early stage of research and development.

This extensive overview of the research in thermoplastic polymer nanocomposites based on IFs provides some insight into the factors that ultimately control their properties. The incorporation of low IF-WS 2 loadings into thermoplastic matrices has been shown to be more efficient for simultaneously improving the thermal, mechanical and tribological properties of the polymer than the addition of similar or even higher amounts of other inorganic nanoparticles. Moreover, the combination of inorganic fullerenes with other organic micro-particles (nucleating agents), micro-fibers (carbon fibers) or nanofillers (carbon nanotubes) allows tailoring of more sophisticated hybrid materials with complex architectures, interactions, morphology and functionality. The results obtained are very promising and suggest that the use of IF-WS 2 , a cheap and environmentally-friendly reinforcing filler, can provide an effective balance between performance, cost effectiveness and processability, making the resulting polymer nanocomposites highly suitable for a wide range of applications at a large scale. Overall, it can be concluded that IFs exhibit promising prospects in the development of new structural and functional materials.

\section{Acknowledgments}

Dr. M. Naffakh would like to acknowledge the Ministerio de Economía y Competitividad (MINECO) for a 'Ramón y Cajal' Senior Research Fellowship and Dr. Ana Diez-Pascual wishes to acknowledge the CSIC for a JAE Postdoctoral Fellowship. This work was also supported by the European Union Sixth Framework Program (FOREMOST project under contract NMP3-CT-2005-515840), the Spanish Ministry of Science \& Innovation, Project MAT-2010-21070-C02-01, the CSIC Intramural Project 201160E003, and the European Commission for the X-ray synchrotron experiments performed at the Soft Condensed Matter A2 beamline at HASYLAB (DESY-Hamburg, Germany).

\section{References}

[1] Okada A, Fukushima Y, Kawasumi M, Inagaki S, Usuki, Sugiyama S, Kurauchi T, Kamigatio O. Composite material and its preparation. US 4,739,007, assigned to Toyota Motor Co, Japan; 1988.

[2] Giannelis EP. Polymer layered silicate nanocomposites. Advanced Materials 1996:8:29-35.

[3] Alexandre M, Dubois P. Polymer-layered silicate nanocomposites: preparation, properties and uses of a new class of materials. Materials Science and Engineering 2000;28:1-63.
[4] Sanchez C, Julián B, Belleville P, Popall M. Applications of hybrid organic-inorganic nanocomposites. Journal of Materials Chemistry 2005; 15:3559-92.

[5] Pavlidou S, Papaspyrides CD. A review on polymer-layered silicate nanocomposites. Progress in Polymer Science 2008;33:1119-98.

[6] Sanchez C, Belleville P, Polpall M, Nicole L. Applications of advanced hybrid organic-inorganic nanomaterials: from laboratory to market. Chemical Society Reviews 2011:40:696-753.

[7] Rozenberg BA, Tenne R. Polymer-assisted fabrication of nanoparticles and nanocomposites. Progress in Polymer Science 2008;33:40-112.

[8] Winey K, Vaia RA. Polymer nanocomposites. MRS Bulletin 2007:32:314-9.

[9] Wang C, Guo ZX, Fu S, Wu W, Zhu D. Polymers containing fullerene or carbon nanotube structures. Progress in Polymer Science 2004:29:1079-141.

[10] Moniruzzaman M, Winey KI. Polymer nanocomposites containing carbon nanotubes. Macromolecules 2006;39:5194-205.

[11] Rahman A, Ali I, AI Zahrani SM, Eleithy RH. A review of the applica tions of nanocarbon polymer composites. Nano 2011;6:185-203.

[12] Kuilla T, Bhadra S, Yao D, Kim NH, Bose S, Lee JH. Recent advances in graphene based polymer composites. Progress in Polymer Science 2010;35:1350-75.

[13] Caseri W. Inorganic nanoparticles as optically effective additives for polymers. Chemical Engineering Communications 2009; 196:549-72.

[14] Li S, Lin MM, Toprak MS, Kim DK, Muhammed M. Nanocomposites of polymer and inorganic nanoparticles for optical and magnetic applications. Nano Reviews 2010;1:5214/1-19, http://dx.doi.org/10.3402/nano.v1 i0.5214.

[15] Jeon IY, Baek JB. Nanocomposites derived from polymers and inorganic nanoparticles. Materials 2010;3:3654-74.

[16] Neoh KG, Kang ET. Functionalization of inorganic nanoparticles with polymer for stealth biomedical applications. Polymer Chemistry 2011:2:747-59.

[17] Kroto HW, Heat JR, O'Brien SC, Curl RF, Smalley RE. C-60Buckminsterfullerene. Nature 1985;318:162-3.

[18] Herbard AF. Superconductivity in doped fullerenes. Physics Today 1992; 45:26-32.

[19] Culotta L, Knoshland DE. Buckyballs: wide open playing field for chemists. Science 1991;254:1706-9.

[20] Olah GA, Bucsi I, Lambert C, Aniszfeld R, Trivedi N], Sensharma DK, Prakash GKS. Polyarenefullerenes, $\mathrm{C} 60(\mathrm{H}-\mathrm{Ar}) \mathrm{n}$, obtained by acidcatalyzed fullerenation of aromatics. Journal of American Chemical Society $1991 ; 113: 9385-7$.

[21] Martín N, Giacalone F. Fullerenes polymers: synthesis: properties and applications. Weinheim: Wiley-VCH; 2009, $332 \mathrm{pp}$.

[22] York APE. Inorganic fullerenes, onions, and tubes. Journal of Chemical Education 2004:81:673-6.

[23] Bar-Sadan M, Kaplan-Ashiri I, Tenne R. Inorganic fullerenes and nanotubes: wealth of materials and morphologies. European Physical Journal Special Topics 2007;149:71-101.

[24] Tenne R. Inorganic nanotubes and fullerene-like nanoparticles. Nature Nanotechnology 2006;1:103-11.

[25] Tenne R, Seifert G. Recent progress in the study of inorganic nanotubes and fullerene-like structures. Annual Review of Materials Research 2009;39:387-413.

[26] Tenne R, Redlich M. Recent progress in the research of inorganic fullerene-like nanoparticles and inorganic nanotubes. Chemical Society Reviews 2010;39:1423-34.

[27] Tenne R, Margulis L, Genut M, Hodes G. Polyhedral and cylindrical structures of WS 2 . Nature 1992;360:444-5.

[28] Margulis L, Salitra G, Tenne R, Talianker M. Nested fullerene-like structures. Nature 1993;365:113-4.

[29] Tenne R. Inorganic nanotubes and fullerene-like materials. Chemistry-A European Journal 2002;8:5297-304.

[30] Dickinson RG, Pauling L. The crystal structure of molybdenite. Journal of American Chemical Society 1923;45:1466-71.

[31] Prasad S, Zabinski J. Super slippery solids. Nature 1997;387:761-3.

[32] Prasad SV, Zabinski JS. Tribology of tungsten disulphide $\left(\mathrm{WS}_{2}\right)$ : characterization of wear-induced transfer films. Journal of Materials Science Letters 1993;11:1413-5.

[33] Rapoport L, Bilik Y, Feldman Y, Homyonfer M, Cohen SR, Tenne R. Hollow nanoparticles of $\mathrm{WS}_{2}$ as potential solid-state lubricants. Nature 1997:387:791-3.

[34] Tevet O, Von-Huth P, Popovitz-Biro R, Rosentsveig R, Wagner HD, Tenne R. Friction mechanism of individual multilayered nanoparticles. Proceedings of the National Academy of Sciences United Status of America 2011;108:19901-6. 
[35] Zhu YQ, Sekine T, Li YH, Wang WX, Fay MW, Edwards H, Brown PD, Fleischer N, Tenne R. WS 2 and $\mathrm{MoS}_{2}$ inorganic fullerenes-super shock absorbers at very high pressures. Advanced Materials 2005:17:1500-3.

[36] Zhu YQ, Sekine T, Van Li H, Fay MW, Zhao YM, Poa CHP, Wang WX, Roe MJ, Brown PD, Fleischer N, Tenne R. Shock-Absorbing and failure mechanisms of $\mathrm{WS}_{2}$ and $\mathrm{MoS}_{2}$ nanoparticles with fullerenelike structures under shock wave pressure. Journal of American Chemical Society 2005;127:16263-72.

[37] Frey GL, Tenne R, Matthews MJ, Dresselhaus MS, Dresselhaus G, Optical properties of $\mathrm{MS}_{2}(\mathrm{M}=\mathrm{Mo}, \mathrm{W})$ inorganic fullerene-like and nanotube material optical absorption and resonance Raman measurements. Journal of Materials Research 1998;13:2412-7.

[38] Tevet O, Goldbart O, CohenSR, Rosentsveig R, Popovitz-Biro R, Wagner HD, Tenne R. Nanocompression of individual multilayered poly hedral nanoparticles. Nanotechnology 2010;21:365705/1-365705.

[39] Kaplan-Ashiri I, Cohen SR, Gartsman K, Rosentsveig R, Seifert G, Tenne R. Mechanical behavior of individual $\mathrm{WS}_{2}$ nanotubes. Journal of Materials Research 2004;19:454-9.

[40] Lahouij I, Dassenoy F, Vacher B, Martin JM. Real time TEM imaging of compression and shear of single fullerene-like $\mathrm{MoS}_{2}$ nanoparticle. Tribology Letters 2012;45:131-41.

[41] Frey GL, Ilani S, Homoyonfer M, Feldman Y, Tenne R. Opticalabsorption spectra of inorganic fullerenelike $\mathrm{MS}_{2}(\mathrm{M}=\mathrm{Mo}, \mathrm{W})$ Physical Review B 1998;57:6666-71.

[42] Seifert G, Terrones H, Terrones M, Jungnickel G, Frauenheim T. On the electronic structure of $\mathrm{WS}_{2}$ nanotubes. Solid State Communications 2000;114:245-8.

[43] Kopnov F, Yoffe A, Leitus G, Tenne R. Transport properties of fullerene-like $\mathrm{WS}_{2}$ nanoparticles. Physica Status Solidi B 2006:243:1229-40.

[44] Feldman Y, Frey GL, Homyonfer M, Lyakhovitskaya V, Margulis I Cohen H, Hodes G, Hutchison JL, Tenne R. Bulk synthesis of inorganic fullerene-like $\mathrm{MS}_{2}(\mathrm{M}=\mathrm{Mo}, \mathrm{W})$ from the respective trioxides and the reaction mechanism. Journal of American Chemical Society $1996 ; 118: 5362-7$.

[45] Feldman $Y$, Zak A, Popovitz-Biro R, Tenne $R$. New reactor for production of tungsten disulfide hollow onion-like (inorganic fullerene-like) nanoparticles. Solid State Science 2000:2:663-72.

[46] Yang H, Liu S, Li J, Li M, Peng G, Zou G. Synthesis of inorganic fullerene-like $W_{2}$ nanoparticles and their lubricating performance. Nanotechnology 2006;17:1512-9.

[47] Chhowalla M, Amaratunga GAI. Thin films of fullerene-like $\mathrm{MoS}_{2}$ nanoparticles with ultra-low friction and wear. Nature 2000;407:164-7.

[48] Sen R, Govindaraj A, Suennaga K, Suzuki S, Kataura H, Iijima S, Achiba Y. Encapsulated and hollow closed-cage structures of WS and $\mathrm{MoS}_{2}$ prepared by laser ablation at $450-1050^{\circ} \mathrm{C}$. Chemical Physics Letters 2001;340:242-8.

[49] Poisot M, Bensch W. Decomposition of tetraalkylammonium thiotungstates characterized by thermoanalysis, mass spectrometry, $\mathrm{x}$-ray diffractometry and scanning electron microscopy. Thermochimica Acta 2007:453:42-51.

[50] Berntsen N, Gutjahr T, Loeffler L, Gomm JR, Seshadri R, Tremel W. A solvothermal route to sigh-surface-area nanostructured $\mathrm{MoS}_{2}$. Chemistry of Materials 2003;15:4498-502.

[51] Mastai Y, Homyonfer M, Gedanken A, Hodes G. Room temperature sonoelectrochemical synt hesis of Molybdenum Sulphide fullerenelike nanoparticles. Advanced Materials 1999;11:1010-3.

[52] Wu H, Yang R, Song B, Han Q, Li J, Zhang Y, Fang Y, Tenne R, Wang C. Biocompatible inorganic fullerene-like molybdenum disulfide nanoparticles produced by pulsed laser ablation in water. ACS Nano 2011:5:1276-81.

[53] Rothschild A, Sloan], Tenne R. The growth of WS 2 nanotubes phases. Journal of American Chemical Society 2000;122:5169-79.

[54] Kickelbick G. Concepts for the incorporation of inorganic building blocks into organic polymers on a nanoscale. Progress in Polymer Science 2003;28:83-114.

[55] Brinker CJ, Scherer GW. Sol-gel science: the physics and chemistry of sol-gel processing. Boston: Academic Press; 1990, 912 pp.

[56] Gómez-Romero P, Sanchez C. Front Matter. In: Gómez-Romero P, Sanchez C, editors. Functional hybrid materials. Weinheim: WileyVCH; 2004. p. i-Xvii.

[57] Naffakh M, Díez-Pascual AM, Marco C, Gómez MA, Jiménez I. Novel melt-processable poly(ether ether ketone) (PEEK)/inorganic fullerene-like $\mathrm{WS}_{2}$ nanoparticles for critical applications. Journal of Physical Chemistry B 2010;114:11444-53.

[58] Hou X, Shan CX, Choy KL. Microstructures and tribological properties of PEEK-based nanocomposite coatings incorporating inorganic fullerene-like nanoparticles. Surface and Coatings Technology 2008;202:2287-91.

[59] Rapoport L, Nepomnyashcy O, Verdyan A, Popovitz-Biro R, Volovik $\mathrm{Y}$, Ittah B, Tenne R. Polymer nanocomposites with fullerene-like solid lubricant. Advanced Engineering Materials 2004;6:44-8.

[60] Buchman A, Dodiuk-Kenig H, Dotan A, Tenne R, Kenig S. Toughening of epoxy adhesives by nanoparticles. Journal of Adhesion Science and Technology 2009:23:753-68.

[61] Shneider M, Dodiuk H, Kenig S, Tenne R. The effect of tung sten sulfide fullerene-like nanoparticles on the toughness of epoxy adhesives. Journal of Adhesion Science and Technology 2010;24:1083-95.

[62] Diéz-Pascualt AM, Naffakh M, Marco C, Ellis G, Gómez-Fatou MA High-performance nanocomposites based on polyetherketones. Progress in Materials Science 2012;57:1106-90.

[63] Suryanarayana $\mathrm{C}$, Froes FH. The structure and mechanical properties of metallic nanocrystals. Metallurgical Transactions A 1992:23:1071-81.

[64] Wang Y, Juang IS. Single screw extrusion compounding of particulate filled thermoplastics: state of dispersion and its influence on impact properties. Journal of Applied Polymer Science 1996;60:1779-91.

[65] Jana SC, Jain S. Dispersion of nanofillers in high performance polymers using reactive solvents as processing aids. Polymer 2001;42:6897-905.

[66] Wu CI, Zhang MQ, Rong MZ, Friedrich K. Tensile performance improvement of low nanoparticles filled-polypropylene composites. Composites Science and Technology 2002;62:1327-40.

[67] Subodh G, Deepu V, Mohanan P, Sebastian MT. Dielectric response of high permittivity polymer ceramic composite with low loss tan gent. Applied Physics Letters 2009:95:062903/1-62903.

[68] Dang Z, Wu J, Fan L, Nan C. Dielectric behavior of Li and Ti codoped NiO/PVDF composites. Chemical Physics Letters 2003;376: $389-94$.

[69] Goyal RK, Jagadale PA, Mulik UP. Thermal, mechanical, and dielectric properties of polystyrene/expanded graphite nanocomposites. Journal of Applied Polymer Science 2009;111:2071-7.

[70] Afzal AB, Akhtar M], Nadeem M, Hassan MM. Invest igation of struc tural and electrical properties of polyanuline/gold nanocomposites. Journal of Physical Chemistry C 2009;113:17560-5.

[71] Huang XY, Jiang PK, Kim CU. Electrical properties of polyethylene/aluminum nanocomposites. Journal of Applied Physics 2007;102:124103/1-124103.

[72] Parvatikar N, Ambika Prasad MVN. Frequency-dependent conductivity and dielectric permittivity of polyaniline $/ \mathrm{CeO}_{2}$ composites. Journal of Applied Polymer Science 2006;100:1403-5.

[73] Badheka P, Magadala V, Gopi Devaraju N, Lee BI, Kim ES Effect of dehydroxylation barium titanate on dielectric properties in polystyrene composite. Journal of Applied Polymer Science 2006:99:2815-21.

[74] Xie S, Zhu B, Xu Z, Xu Y. Preparation and properties of polyamide/LTNO composite films with high dielectric constant Materials Letters 2005:59:2403-7.

[75] Schroeder R, Majewski L, Grell M. High-performance organic transistors using solution-processed nanoparticle-filled high-kpolymer gate insulators. Advanced Materials 2005;17:1535-9.

[76] Andou Y, Jeong JM, Nishida H, Endo T. Simple procedeure for polystyrene-based nanocomposites preparation by vapor-phaseassisted surface polymerization. Macromolecules 2009;42:7930-5.

[77] Thomas P, Dwarakanath K, Varma KBR. In situ synthesis and characterization of polyaniline- $\mathrm{CaCu}_{3} \mathrm{Ti}_{4} \mathrm{O}_{12}$ nanocrystal composites. Synthetic Metals 2009;159:2128-34

[78] Reynaud E, Jouen T, Gauthier C, Vigier G, Varlet J. Nanofillers in polymeric matrix: a study on silica a reinforced PA6. Polymer 2001;42:8759-68.

[79] Van Zyl WE, García M, Schrauwen BAG, Kooi BJ, De Hosson JM Verweij H. Hybrid polyamide-silica nanocomposites: synthesis and mechanical testing. Macromolecular Materials and Engineering 2002:287:106-10.

[80] He A, Wang L, Li], Dong], Han CC. Preparation of exfoliated isotactic polypropylene/alkyl-triphenylphosphonium-modied montmorillonite nanocomposites via in situ intercalative polymerization. Polymer 2006:47:1767-71.

[81] Ginzburg VV, Myers K, Malowinski S, Cieslinski R, Elwell M, Bernius M. High-dielectric-constant self-assembled nodular structures in polymer/gold nanoparticle films. Macromolecules 2006:39:3901-6.

[82] Mizutani T, Arai K, Miyamoto M, Kimura Y. Preparation of spheri cal nanocomposites consisting of silica core and polyacrylate shel 
by emulsion polymerization. Journal of Applied Polymer Science 2006:99:659-69.

[83] Xiao M, Sun L, Liu J, Li Y, Gong K. Synthesis and properties of polystyrene/graphite nanocomposites. Polymer 2002;43:2245-8.

[84] Yang F, Ou Y, Yu ZZ. Polyamide 6/silica nanocomposites prepared by in situ polymerization. Journal of Applied Polymer Science 1998;69:355-61.

[85] Ou Y, Yang F, Yu ZZ. A new conception on the toughness of nylon 6/silica nanocomposite prepared via in-situ polymerization. Journal of Polymer Science Part B Polymer Physics 1998;36: $789-95$

[86] Balan L, Jin M, Malval JP, Chaumeil H, Defoin A, Vidal L. Fabrication of silver nanoparticle-embedded polymer promoted by combined photochemical properties of a 2,7-diaminofluorene derivative dye. Macromolecules 2008;41:9359-65.

[87] Lu J, Moon KS, Xu J, Wong CP. Synthesis and dielectric properties of novel high-k polymer composites containing in-situ formed silver nanoparticles for embedded capacitor applications. Journal of Materials Chemistry 2006;16:1543-8.

[88] Yogo T, Yamamoto T, Sakamoto W. Hirano S. In situ synthesis of nanocrystalline $\mathrm{BaTiO}_{3}$ particle-polymer hyvrid. Journal of Materials Research 2004; 19:3290-7.

[89] Vaia RA, Maguire JF. Polymer nanocomposites with prescribed morphology: going beyon nanoparticle-filled polymers. Chemistry of Materials 2007; 19:2736-51.

[90] Mackay ME, Tuteja A, Duxbury PM, Hawker CJ, Van Horn B, Guan Z, Chen G, Krishnan RS. General st rategies for nanoparticle dispersion. Science 2006:311:1740-3.

[91] Lin Y, Boeker A, He J, Sill K, Xiang H, Abetz C, Li X, Wang J, Emrick T, Long S, Wang Q, Balazs A, Russell TP. Self-directed self-assembly of nanoparticle/copolymer mixtures. Nature 2005;434:55-9.

[92] Chandra A, Turng IS, Gopalan P, Rowell RM, Gong S. Study of utilizing thin polymer surface coating on the nanoparticles for melt compounding of polycarbonate/alumina nanocomposites and their optical properties. Composites Science and Technology 2008;68:768-76.

[93] Naffakh M, Martin Z, Fanegas N, Marco C, Gómez MA, Jiménez I. Influence of inorganic fullerene-like $\mathrm{WS}_{2}$ nanoparticles on the thermal behavior of isotactic polyp ropylene. Journal of Polymer Science Part B Polymer Physics 2007;45:2309-21.

[94] Vassiliou A, Bikiaris D, Pavlidou E. Optimizing melt-processing conditions for the preparation of iPP/fumed silica nanocomposites: morphology, eechanical and gas permeability properties. Macromolecular Reaction Engineering 2007;1:488-501.

[95] Asuka K, Liu B, Terano M, Nitta K. Homogeneously dispersed poly(propylene)/SiO2 nanocomposites with unprecedented transparency. Macromolecular Rapid Communications 2006;27:910-3.

[96] Zhang S, Zhu A, Dai S. Coincorporation of nano-silica and nanocalcium carbonate in polypropylene. Journal of Applied Polymer Science 2011:121:3007-13.

[97] Kruenate J, Tongpool R, Panyathanmaporn T, Kongrat P. Optical and mechanical properties of polypropylene modified by metal oxides. Surface and Interface Analysis 2004;36:1044-7.

[98] Papageorgiou GZ, Achilias DS, Bikiaris DN, Karayannidis GP. Crystallization kinetics and nucleation activity of filler in polypropylene/surface-treated $\mathrm{SiO}_{2}$ nanocomposites. Thermochimica Acta 2005;427:117-28.

[99] Nitta K, Asuka K, Liu B, Terano M. The effect of the addition of silica particles on linear spherulite growth rate of isotactic polypropylene and its explanation by lamellar cluster model. Polymer 2006:47:6457-63.

[100] Chen J, Yang MS, Zhang SM. Immobilization of antioxidant on nanosilica and the aging resistance behaviour in polypropylene. Composites Part A Applied Science and Manufacturing 2011:42:471-7.

[101] Donato RK, Benvegnu MA, Furlan LG, Mauler RS, Schrekker HS. Imidazolium salts as liquid coupling agents for the preparation of polypropylene-silica composites. Journal of Applied Polymer Sci ence $2010: 116: 304-7$.

[102] Bikiaris DN, Vassiliou A, Pavlidou E, Karayannidis GP. Compat ibilisation effect of PP-g-MA copolymer on iPP/SiO2 nanocomposites prepared by melt mixing. European Polymer Journal 2005:41:1965-78.

[103] Tan X, Xu Y, Cai N, Jia G. Polypropylene/silica nanocomposites prepared by in-situ melt ultrasonication. Polymer Composites 2009;30:835-40.

[104] Palza H, Vergara V, Zapata P. Composites of polypropylene melt blended with synthesized silica nanoparticles. Composites Science and Technology 2011:71:535-40.
[105] Palza H, Vera J, Wilhelm M, Zapata P. Spherulite growth rate in polypropylene/silica nanoparticle composites: effect of particle morphology and compatibilizer. Macromolecular Materials and Engineering 2011;296:744-51.

[106] Palza $H$, Vergara $R$, Zapata P. Improving the thermal behavior of poly(propylene) by addition of spherical silica nanoparticles. Macromolecular Materials and Engineering 2010;295: 899-905.

[107] Kim KD, Kim HT. Formation of silica nanoparticles by hydrolysis of TEOS using a mixed semi-batch/batch method. Journal of Sol-Gel Science Technology 2002;25:183-9.

[108] Rong MZ, Zhang MQ, Zheng YX, Zeng HM, Walter R, Friedrich K. Irradiation graft polymerization on nano-inorganic particles: an effective means to design polymer-based nanocomposites. Journal of Materials Science Letters 2000;19:1159-61.

[109] Wu CL, Zhang MQ, Rong MZ, Friedrich K. Silica nanoparticles filled polypropylene: effects of particle surface treatment, matrix ductility and particle species on mechanical performance of the composites. Composites Science and Technology 2005;65: $635-45$.

[110] Rong MZ, Zhang MQ, Pan SL, Friedrich K. Interfacial effects in polypropylene-silica nanocomposites. Journal of Applied Polymer Science 2004:92:1771-81.

[111] Zheng JZ, Zhou XP, Ying JR, Xie XL, Mai YW. Enahanced mechanical properties of polypropylene/silica nanocomposites with surface modification of nano-silica via insitu copolymerization of methyl methacrylate ande butyl acrylate. Chinese Journal of Polymer Science 2009;27:685-94.

[112] Ruan WH, Zhang MQ, Rong MZ, Friedrich K. Polypropylene composites filled with in-situ grafting polymerization modified nano-silica particles. Journal of Materials Science 2004;39:3475-8.

[113] Cai LF, Mai YL, Rong MZ, Ruan WH, Zhang MQ. Interfacial effects in nano-silica/polypropylene composites fabricated by in-situ chemical blowing. Polymer Letters 2007:1:2-7.

[114] Lin OH, Akil HM, Ishak ZAM. Comparative study between PPg-MAH and PP-methyl-POSS as compatibilizer for nanoSiO $2 / \mathrm{PP}$ composites: mechanical and morphological properties. Advanced Composites Letters 2009;18:183-90.

[115] Zhou HJ, Rong MZ, Zhang MQ, Ruan WH, Friedrich K. Role of reactive compatibilization in preparation of nanosilica/polypropylene composites. Polymer Engineering \& Science 2007;47:499-509.

[116] Wan W, Yu D, Xie Y, Guo X, Zhou W, Cao J. Effects of nanoparticle treatment on the crystallization behavior and mechanical properties of polypropylene/calcium carbonate nanocomposites. Journal of Applied Polymer Science 2006:102:3480-8

[117] Karamipour S, Ebadi-Dehaghani H, Ashouri D, Mousavian S. Effect of nano- $\mathrm{CaCO}_{3}$ on rheological and dynamic mechanical properties of polypropylene: experiments and models. Polymer Testing 2011;30:110-7

[118] Wang G, Chen XY, Huang R, Zhang L. Nano-CaCO $/$ /polypropylene composites made with ultra-high-speed mixer. Journal of Materials Science Letters 2002;21:985-6.

[119] Meng MR, Dou Q. Effect of filler treatment on crystallization, morphology and mechanical properties of polypropylene/calcium carbonate composites. Journal of Macromolecular Science Part B Physics 2009:48:213-25.

[120] Liu ZY, Yu RZ, Yang MB, Feng JM, Yang W, Yin B. An approach to preparation of polymer nano $\mathrm{CaCO}_{3}$ composites. Acta Polymerica Sinica 2007:1:53-8.

[121] Tao R, Liu ZY, Yang W, Yang MB. Effect of dispersión condition of calcium carbonate on the crystallization and meeting behaviour of polypropylene $/ \mathrm{CaCO}_{3}$ nanocomposites. Polymer Plastics and Technology Engineering 2008;47:490-5.

[122] Yang K, Yang O, Li G, Sun Y, Feng D. Mechanical properties and morphologies of polypropylene with different sizes of calcium carbonate particles. Polymer Composites 2006;27:443-50.

[123] Yang K, Yang Q, Li GX, Sun YJ, Feng DC. Morphology and mechanical properties of polypropylene/calcium carbonate nanocomposites. Materials Letters 2006:60:805-9.

[124] Xu GC, Wang J, Ji XL, Xiong JY, Li F. Effect of nano-silicon nitride on the mechanical and electric properties of polypropylene nanocomposite. Journal of Composite Materials 2007:41:2213-23.

[125] López V, Román-Pérez G, Arregui A, Mateo-Marti E, Bañares L, Martín-GagojA, Soler JM, Gómez-HerreroJ, Zamora F. Azafullerenelike nanosized clusters. ACS Nano 2009:3:3352-7.

[126] Naffakh M, López V, Zamora F, Gómez MA. Novel melt-processable nanocomposites based on isotactic polypropylene and carbon nit ride: morphology, crystallization and dinamic mechanical properties. Soft Materials 2010;8:407-25. 
[127] Tang J, Wang Y, Liu H, Belfiore LA. Effects of organic agents and zinc oxide nanoparticles on isotactic polypropylene crystallization. Polymer 2004;45:2081-91.

[128] Zhao H, Li RKY. A study on the photo-degradation of zinc oxide (ZnO) filled polypropylene nanocomposites. Polymer 2006:47:3207-17

[129] Huang CK, Chen SW, Wei WCJ. Processing and property improvement of polymeric composites with added Zno nanoparticles through microinjection molding. Journal of Applied Polymer Sci ence 2006:102:6009-16.

[130] Lepot N, Van-Bael MK, Van-den-Rul H, D'Haen J, Peeters R, Franco $\mathrm{D}$, Mullens J. Influence of incorporation of ZnO nanoparticles and biaxial orientation on mechanical and oxygen barrier properties of polypropylene films for food packaging applications. Journal of Applied Polymer Science 2011;120:1616-23.

[131] Majid M, Hassan ED, Davoud A, Saman M. A study on the effect of nano-ZnO on rheological and dynamic mechanical properties of polypropylene: experiments and models. Composites Part B Engineering 2011;42:2038-46.

[132] Wheeler PA, Misra R, Cook RD, Morgan SE. Polyhedral oligomeric silsesquioxane trisilanols as dispersants for titanium oxide nanopowder. Journal of Applied Polymer Science 2008;108:2503-8.

[133] Misra R, Fu B, Morgan SE. Surface energetics, dispersion, and nanotribomechanical behaviour of POSS/PP hybrid nanocomposites. Journal of Polymer Science Part B Polymer Physics 2007:45:2441-55.

[134] Zhao H, Robert K, Li Y. Crystallization, mechanical, and fracture behaviors of spherical alumina-filled polypropylene nanocomposites. Journal of Polymer Science Part B Polymer Physics 2005:43:3652-64

[135] Ford WT, Lary AL, Mourey TH. Addition of polystyryl radicals from TEMPO-terminated polystyrene to $\mathrm{C}_{60}$. Macromolecules 2001;34:5819-26

[136] Okamura H, Terauchi T, Minoda M, Fukuda T, Komatsu K. Synthesis of 1,4-dipolystyryldihydrol60]fullerenes by using $2,2,6,6$ tetramethyl-1-polystyroxypiperidine as a radical source. Macromolecules 1997;30:5279-84

[137] Audouin F, Nuffer R, Mathis C. Synthesis of di- and tetra-adducts by addition of polystyrene macroradicals onto fullerene $C_{60}$. Journal of Polymer Science Part A Polymer Chemistry 2004;42: 3456-63.

[138] Ford WT, Graham TD, Mourey TH. Incorporation of $\mathrm{C}_{60}$ into poly(methyl methacrylate) and polystyrene by radical chain polymerization produces branched structures. Macromolecules 1997:30:6422-9.

[139] Ford WT, Nishioka T, Mccleskey SC, Mourey TH, Kahol P. Structure and radical mechanism of formation of copolymers of $C_{60}$ with styrene and with methyl methacrylate. Macromolecules 2000:33:2413-23

[140] Cao T, Webber SE. Free-radical copolymerization of fullerenes with styrene. Macromolecules 1995:28:3741-3.

[141] Martinez G, Gomez MA Gomez R, Segura JL Synthesis of a [60]fullerene-functionalized Poly(vinyl chloride) derivative by stereospecific Chemicals modification of PVC. Journal of Polyme Science Part A Polymer Chemistry 2007;45:5408-19.

[142] Martinez G, Gomez MA, Marco C, Ellis G, Gomez R, Segura JL. Synthesis of a [60]fullerene isotactic polypropylene derivative. Journal of Polymer Science Part A Polymer Chemistry 2008;46:6722-33.

[143] Wan D, Zhang Z, Wang Y, Xing H, Jiang Z, Tang T. Dependence of microstructures and melt behaviour of polypropylene/fullerene C60 nanocomposites on in situ interfacial reaction. Soft Matter 2011:7:5290-9.

[144] Naffakh M, Marco C, Gômez MA, Jiménez I. Novel melt-processable nylon-6/inorganic fullerene-like WS2 nanocomposites: complex isothermal crystallization kinetics and melting behaviour. Mate rials Chemistry and Physics 2011;128:265-73.

[145] Shen L, Du Q, Wang H, Zhong W, Yang Y. In situ polymeriza tion and characterization of polyamide-6/silica nanocomposites derived from water glass. Polymer International 2004;53:1153-60.

[146] Avella M, Bondioli F, Cannillo V, Errico ME, Ferrari AM, Focher B, Melinconico M, Manfredini T, Montorsi M. Preparation, characterisation and computacional study of Poly( $\varepsilon$ caprolactame) based nanocomposites. Materials Science and Technology 2004;20:1340-4

[147] Cannillo V, Bondioli F, Lusvarghi L, Montorsi M, Avella M, Errico ME, Malinconico M. Modeling of ceramic particles filled polymer-matrix nanocomposites. Composites Science and Technology 2006;66:1030-7.
[148] Rusu G, Rusu E. Nylon $6 / \mathrm{SiO}_{2}$ nanocomposites synthesized by in situ polymerization. High Performance Polymers 2006;18:355-75.

[149] Cai LF, Lin ZY, Qian H. Dispersion of nano-silica in monomer casting nylon 6 and its effect on the structure and properties of composites Express Polymer Letters 2010;4:397-403.

[150] Goyal RK, Tiwari AN, Mulik UP, Negi YS. Novel high performance $\mathrm{Al}_{2} \mathrm{O}_{3}$ /poly(ether ether ketone) nanocomposites for electronics applications. Composites Science and Technology 2007;67: $1802-12$.

[151] Kuo MC, Huang JC, Chen M. Non-isothermal crystallization kinetic behavior of alumina nanoparticle filled poly(ether ether ketone) Materials Chemistry and Physics 2006:99:258-68.

[152] Kuo MC, Tsai CM, Huang IC, Chen M. PEEK composites reinforced by nano-sized $\mathrm{SiO}_{2}$ and $\mathrm{Al}_{2} \mathrm{O}_{3}$ particulates. Materials Chemistry and Physics 2005;90:185-95.

[153] Balaji V, Tiwari AN, Goyal RK. Fabrication and properties of high performance PEEK/Si $3 \mathrm{~N}_{4}$ nanocomposites. Journal of Applied Polymer Science 2011:119:311-8.

[154] Bikiaris DN, Papageorgiou GZ, Pavlidou E, Vouroutzis N, Palatzoglou $\mathrm{P}$, Karayannidis GP. Preparation by melt mixing and characterization of isotactic polypropylene/ $\mathrm{SiO}_{2}$ nanocomposites containing untreated and surface-treated nanoparticles. Journal of Applied Polymer Science 2006;100:2684-96.

[155] Kuo MC, Kuo JS, Yang MH, Huang JC. On the crystallization behavior of the nano-silica filled PEEK composites. Materials Chemistry and Physics 2010;123:471-80.

[156] Jordan J, Jacob KI, Tannenbaum R, Sharaf MA, Jasiuk I. Experimental trends in polymer nanocomposites-a review. Materials Science and Engineering A 2005:393:1-11.

[157] Zou H, Wu S, Shen J. Polymer/silica nanocomposites: preparation, characterization, properties, and applications. Chemical Reviews 2008;108:3893-957.

[158] Hussain F, Hojjati M, Okamoto M, Gorga RE. Polymer-matrix nanocomposites, processing, manufacturing, and application: an overview. Journal of Composites Materials 2006;40:1511-75.

[159] Lai YH, Kuo MC, Huang IC, Chen M. On the PEEK composites reinforced by surface-modified nano-silica. Materials Science and Engineering A 2007;458:158-69.

[160] Sun Y, Zhang Z, Wong CP. Study on mono-dispersed nano-size silica by surface modification for underfill applications. Journal of Colloid and Interface Science 2005;292:436-44.

[161] Hedayati M, Salehi M, Bagheri R, Panjepour M, Maghzian A Ball milling preparation and characterization of poly (ether ether ketone)/surface modified silica nanocomposite. Powder Technology 2011:207:296-303.

[162] Naffakh M, Marco C, Gomez MA, Jimenez I. Unique isothermal crystallization behavior of novel polyphenylene sulfide/inorganic fulle rene-like $\mathrm{WS}_{2}$ nanocomposites. Journal of Physical Chemistry B 2008;112:14819-28.

[163] Lu D, Mai YW, Robert K, Li Y, Ye L. Impact strength and crystallization behavior of nano-SiOx/poly(phenylene sulfide) (PPS) composites with heat-treated PPS. Macromolecular Materials and Engineering 2003;288:693-8.

[164] Lu D, Pan S. Effects of ball milling dispersion of nano-Siox particles on impact strength and crystallization behavior of nano-SiOx poly(phenylene sulfide) nanocomposites. Polymer Engineering \& Science 2006;46:820-5

[165] Hu KH, Wang J, Schraube S, Xu YF, Hu XG, Stengler R. Tribological properties of $\mathrm{MoS}_{2}$ nano-balls as filler in polyoxymethylene-based composite layer of three-layer self-lubrication bearing materials. Wear 2009:266:1198-207.

[166] Hu H, Wang YR, Hu XG, Wo HZ. Preparation and characterisation of ball like $\mathrm{MoS}_{2}$ nanoparticles. Materials Science and Technology 2007:23:242-6.

[167] Naffakh M, Marco C, Gomez MA, Jimenez I. Novel melt-processable nylon-6/inorganic fullerene-like $\mathrm{WS}_{2}$ nanocomposites for crit ical applications. Materials Chemistry and Physics 2011;129: $641-8$

[168] Naffakh M, Marco C, Gómez MA, Gómez-Herrero J, Jiménez I. Use of inorganic fullerene-like $\mathrm{WS}_{2}$ to produce new highperformance polyphenylene sulfide nanocomposites: Role of the nanoparticle concentration. Journal of Physical Chemistry B 2009;113:10104-11.

[169] Avella M, Cosco S, Di-Lorenzo ML, Di-Pace E, Errico ME. Influence of $\mathrm{CaCO}_{3}$ nanoparticles shape on thermal and crystallization behaviour of isotatic polypropylene based nanocomposites. Journal of Thermal Analysis and Calorimetry 2005;80:131-6.

[170] Lin OH, Akil HM, Ishak ZAM. Surface-activated nanosilica treated with silane coupling agents/polypropylene composites 
mechanical, morphological, and thermal studies. Polymer composites $2001 \cdot 32: 1568-83$.

[171] Sengupta R, Bandyopadhyay A, Sabharwal S, Chaki TK, Bhowmick AK. Polyamide-6,6/in situ silica hybrid nanocomposites by solgel technique: synthesis, characterization and properties. Polymer 2005:46:3343-54.

[172] Hasan MM, Zhou Y, Mahfuz H, Jeelani S. Effect of SiO2 nanoparticle on thermal and tensile behavior of nylon-6. Materials Science and Engineering A 2006;29:181-8.

[173] Zhang L, Xiong Y, Ou E, Chen Z, Xiong Y, Xu W. Preparation and properties of nylon 6/carboxylic silica nanocomposites via in situ polymerization. Journal of Applied Polymer Science 2011;122:1316-24.

[174] Rusu G, Rusu E. Nylon $6 / \mathrm{TiO}_{2}$ composites by in situ anionic ring-opening polymerization of $\varepsilon$-caprolactam: synthesis, characterization and properties. International Journal of Polymer Analysis and Characterization 2011;16:561-83.

[175] Moussa MA, Ghoeim AM, Hakim AA, Turky GM. Electrical and thermal properties of nylon 6/calcium carbonate composites. Advances in Polymer Technology 2009;28:257-66.

[176] Sonawane SS, Mishra S, Shimpi NG. Effect of nano- $\mathrm{CaCO}_{3}$ on mechanical and thermal properties of polyamide nanocomposites. Polymer-Plastics Technology and Engineering 2010;49:38-44.

[177] Wu D, Wu L, Fei G, Zhang M, Yan C. Poly(phenylene sulfide) magnetic composites. II. Crystallization, thermal, and viscoelastic properties. Polymer Engineering \& Science 2008;48: 966-75.

[178] Goyal RK, Negi YS, Tiwari AN. Preparation of high performance composites based on aluminum nitride/poly(ether-ether-ketone) and their properties. European Polymer Journal 2005;41:2034-44

[179] Balaji V, Balaji V, Tiwari AN, Goyal RK. Study on highperformance poly(etheretherketone)/ $\mathrm{Si}_{3} \mathrm{~N}_{4}$ nanocomposites: new electronic substrate materials. Polymer Engineering \& Science 2011:51:509-17.

[180] Fillon B, Lotz B, Thierry A, Wittmann JC. Self-nucleation and enhanced nucleation of polymers. Definit ion of a convenient calorimetric "efficiency scale" and evaluation of nucleating additives in isotactic polypropylene ( $\alpha$ phase). Journal of Polymer Science Part B Polymer Physics 1993;31:1395-405.

[181] Marco C, Gómez MA, Ellis G, Arribas JM. Activity of different nucleating agents on the crystallization behaviour of isotactic polypropylene and its impacts on some mechanical properties. Recent Research and Development in Applied Polymer Science 2002:1:587-610.

[182] Fillon B, Thierry A, Lotz B, Wittman JC. Efficiency scale for polymer nucleating agents. Journal of Thermal Analysis 1994:42:721-31.

[183] Marco C, Gómez MA, Ellis G, Arribas JM. Highly efficient nucleating additive for isotactic polypropylene studied by differential scanning calorimetry. Journal of Applied Polymer Science 2002:84:1669-79.

[184] Quian J, He P, Nie K. Nonisothermal crystallization of PP/nano-SiO composites. Journal of Applied Polymer Science 2004;91:1013-9.

[185] Jain S, Goossens H, Van-Duin M, Lemstra P. Effect of in situ prepared silica nano-particles on non-isothermal crystallization of polypropylene. Polymer 2005;46:8805-18.

[186] Lin Z, Huang Z, Zhang Y, Mai K, Zeng H. Crystallization and melting behavior of nano- $\mathrm{CaCO}_{3} /$ polypropylene composites modified by acrylic acid. Journal of Applied Polymer Science 2004;91:2443-53.

[187] Petraccone V, Guerra G, De-Rosa C, Tuzi A. Extrapolation to the equilibrium melting temperature for isotactic polypropylene Macromolecules 1985;18:813-4.

[188] Naffakh M, Martín Z, Marco C, Gómez MA, Jiménez I. Isothermal crystallization kinetics of isotactic polypropylene with inorganic fullerene-likeWS ${ }_{2}$ nanoparticles. Thermochimica Acta 2008:472:11-6.

[189] Lauritzen JL, Hoffman JD. Extension of theory of growth of chainfolded polymer crystals to larde undercoolings. Journal of Applied Physics 1973;44:4340-52.

[190] Dougnac VN, Alamillo R, Peoples BC, Quijada R. Effect of particle diameter on the permeability of polypropylene/ $\mathrm{SiO}_{2}$ nanocompos ites. Polymer 2010;51:2918-26.

[191] Huang L, Renbo-Zhan R, Lu Y. Mechanical properties and crystallization behavior of polypropylene/nano- $\mathrm{SiO}_{2}$ composites. Journal of Reinfored Plastics and Composites 2006;25:1001-12.

[192] Vladimirov V, Betchev C, Vassiliou A, Papageorgiou G, Bikiaris D. Dynamic mechanical and morphological studies of isotactic polypropylene/fumed silica nanocomposites with enhanced gas barrier properties. Composites Science and Technology 2006;66: $2935-44$.
[193] Qian J, Cheng G, Zhang H, Xu Y. Preparation and characterization of polypropylene/silica nanocomposites by gamma irradiation via ultrafine blend. Journal of Polymer Research 2011;18:409-17.

[194] Chan C, Wu J, Li JX, Cheung YK. Polypropylene/calcium carbonate nanocomposites. Polymer 2002;43:2981-92.

[195] Mishra S, Sonawane SH, Singh RP. Studies on characterization of nano $\mathrm{CaCO}_{3}$ prepared by the in situ deposition technique and its application in $\mathrm{PP} /$ nano $\mathrm{CaCO}_{3}$ composites. Journal of Polymer Science Part B Polymer Physics 2005;43:107-13.

[196] Eiras D, Pessan LA. Influence of calcium carbonate nanoparticles on the crystallization of polypropylene. Materials Research 2009; $12: 523-7$

[197] Shentu B, Li ], Weng Z. Effect of oleic acid-modified nano- $\mathrm{CaCO}_{3}$ on the crystallization behavior and mechanical properties of polypropylene. Chinese Journal of Chemical Engineering 2006;14:814-8.

[198] Chen H, Wang M, Lin Y, Chan CM, Wu J. Morphology and mechanical property of binary and ternary polypropylene nanocomposites with nanoclay and $\mathrm{CaCO}_{3}$ particles. Journal of Applied Polymer Science 2007:106:3409-16

[199] Gong G, Yang W, Yang M, Wu J. Effect of spherical nanoparticles on the motion of mac romolecular chains and segments of isotactic polypropylene. I. Dynamic mechanical and thermal properties. Journal of Macromolecular Science R Part B Physics 2010;49:870-85.

[200] Avella M, Cosco S, Di-Lorenzo ML, Di-Pace E, Errico ME, Gentile G. Nucleation activity of nanosized $\mathrm{CaCO}_{3}$ on crystallization of isotactic polypropylene, in dependence on crystal modification, particle shape, and coating. European Polymer Journal 2006;42:1548-57.

[201] Li LZ, Zhang XQ, Su YL, Li RB, Zhu HJ, Zhou Y, Zhao Y, Wang D]. Pro bing into double crystallisation behaviour of polypropylene $/ \mathrm{CaCO}_{3}$ composites. Plastics Rubber and Composites 2010;39:425-9.

[202] Zhang QX, Yu ZZ, Xiea XL, Mai YW. Crystallization and impact energy of polypropylene $/ \mathrm{CaCO}_{3}$ nanocomposites with nonionic modifier. Polymer 2004;45:5985-94.

[203] Huang H, Han B, Wang L, Miao N, Mo H, Zhou NL, Ma ZM, Zang], Shen J. Crystallization kinetics of polypropylene composites filled with nano calcium carbonate modified with maleic anhydride. Journal of Applied Polymer Science 2011:119:1516-27.

[204] Wang Y, Shen H, Li G, Mai K. Crystallization and melting behavior of $\mathrm{PP} /$ nano- $\mathrm{CaCO}_{3}$ composites with diffe rent interfacial interaction. Journal of Thermal Analysis and Calorimetry 2010;99:399-407.

[205] D'Haese M, Langouche F, Van-Puyvelde P. Effect of particle presence and flow at processing speeds of polypropylene crystallization. Macromolecules 2010;43:2933-41.

[206] Razdan S, Patra P, Kim Y, Wamer S. Sperulitic morpbology and thermal stability of PP/ZnO nanocomposites. Materials Research Society Symposium Proceedings 2004;788:297-302.

[207] Supaphol P, Thanomkiat P, Junkasem J, Dangtungee R. Nonisothermal melt-crystallization and mechanical properties of titanium (IV) oxide nanoparticle-filled isotactic polypropylene. Polymer Testing 2007;26:20-37.

[208] Rangari VK, Shaik YM, Mohammad GM, Shaik-Jeelani S. Reinforce ment of $\mathrm{Si}_{3} \mathrm{~N}_{4}$ nanoparticles in polypropylene single fibers through melt extrusion process and their properties. Journal of Applied Polymer Science 2011:121:1512-20.

[209] Hao W, Yang W, Cai H, Huang Y. Non-isothermal crystallization kinetics of polypropylene/silicon nitride nanocomposites. Polymer Testing 2010;29:527-33.

[210] Wang XD, Zhang YX, Liu BG, Du ZJ, Li HQ. Crystallization behavior and crystal morphology of linear/long chain branching polypropylene blends. Polymer Journal 2008;40:450-4.

[211] Holmes DR, Bunn CW, Smith D]. The crystal structure of polycaproamide: Nylon 6. Journal of Polymer Science 1955;17:159-77.

[212] Arimoto $H$, Ishibashi M, Hirai M, Chatani Y. Crystal structure of the $\gamma$-form of nylon 6 . Journal of Polymer Science Part A Polymer Chemistry 1965:3:317-26.

[213] Murthy NS, Curran SA, Aharoni SM, Minor H. Premelting crystalline relaxations and phase transitions in nylon 6 and 6,6. Macromolecules 1991;24:3215-20.

[214] Fornes TD, Paul DR. Crystallization behavior of nylon 6 nanocomposites. Polymer 2003;44:3945-61.

[215] Zapata-Espinosa A, Medellín-Rodríguez FJ, Stribeck N, AlmendarezCamarillo A, Vega-Diaz S, Hsiao BS, Chu B. Complex isothermal crystallization and melting behavior of nylon 6 nanoclay hybrids. Macromolecules 2005;38:4246-53.

[216] Brill R. Über das Verhalten von Polyamiden beim erhitzen. Journal für Praktische Chemie 1942;161:49-64.

[217] Rangari VK, Shaik MY, Mahfuz H, Jeelani S. Fabrication and characterization of high strength Nylon-6/Si $\mathrm{N}_{3} \mathrm{~N}_{4}$ polymer nanocomposite fibers. Materials Science and Engineering A 2009;500:92-7. 
[218] Zhang G, Schlarb AK, Tria S, Elkedim O. Tensile and tribological behaviors of PEEK/nano- $\mathrm{SiO}_{2}$ composites compounded using a ball milling technique. Composites Science and Technology 2008;68:3073-80.

[219] Shangankuli VL, Jog JP, Nadkani VM. Thermal and crystallization behavior of engineering polyblends. I. Glass reinforced polyphenylene sulfide with polyethylene terephthalate. Journal of Applied Polymer Science 1988;36:335-51.

[220] Cho MH, BahaduraS, AndereggJW. Design of experiments approach to the study of tribological performance of Cu-concentrate-filled PPS composites. Tribology International 2006;39:1436-46.

[221] Wu DF, Wu LF, Gao F, Zhang M, Yan CH, Zhou WD. Poly(phenylene sulfide) magnetic composites. I. Relations of percolation between rheology, electrical, and magnetic properties. Journal of Polymer Science Part B Polymer Physics 2008;46:233-43.

[222] Zou H, Xu W, Zhang Q, Fu Q, Effect of alkylammonium salt on the dispersion and properties of poly( $p$-phenylene sulfide)/clay nanocomposites via melt intercalation. Journal of Applied Polyme Science 2006:99:1724-31.

[223] Saito T, Okamoto M, Hiroi R, Yamamoto M, Shiroi T. Intercalation of diphenyl sulfide into nanogalleries and preparation of poly(p-phenylenesulfide)-based nanocomposites. Macromolecular Materials and Engineering 2006;291:1367-74.

[224] Cho MH, Bahadur S. A study of the thermal, dynamic mechanical, and tribological properties of polyphenylene sulfide composites reinforced with carbon nanofibers. Tribology Letters 2007:25:237-45.

[225] Zhao YF, Xiao M, Wang SJ, Ge XC, Meng YZ. Preparation and properties of electrically conductive PPS/expanded graphite nanocomposites. Composites Science and Technology 2007;67: 2528-34.

[226] Naffakh M, Marco C, Gómez MA, Jiménez I. Unique nucleation activity of inorganic fullerene-like $\mathrm{WS}_{2}$ nanoparticles in polyphenylene sulfide nanocomposites: Isokinetic and isoconversional study of dynamic crystallization kinetics. Journal of Physical Chemistry B 2009;113:7107-15.

[227] Devaux E, Bourbigot S, El-Achari A. Crystallization behavior of PA6 clay nanocomposite hybrid. Journal of Applied Polymer Science 2002:86:2416-23.

[228] Kim SH, Ahn SH, Hirai T. Crystallization kinetics and nucleation activity of silica nanoparticle-filled poly(ethylene 2,6-naphthalate) Polymer 2003;44:5625-34

[229] Kim JY, Park HS, Kim SH. Unique nucleation of multi-walled carbon nanotube and poly(ethylene 2,6-naphthalate) nanocomposites during non-isothermal crystallization. Polymer 2006;47:1379-89.

[230] Tabor BJ, Magre EP, Boon J. The crystal structure of poly-pphenylene sulphide. European Polymer Journal 1971;7:1127-33.

[231] Ma CM, Hsiue L, Wu W, Liu W. Rheological and morphologica properties of thermal-aged poly(phenylene sulfide) resin. Journal of Applied Polymer Science 1990;39:1399-415.

[232] Hana Z, Fina A. Thermal conductivity of carbon nanotubes and their polymer nanocomposites: a review. Progress in Polymer Science 2011:36:914-44.

[233] Ohashi M, Kawakami S, Yokogawa Y. Spherical aluminum nitride fillers for heat-conducting plastic packages. Journal of American Ceramic Society 2005;88:2615-8.

[234] Yu S, Hing P, Hu X. Thermal conductivity of polystyrene-aluminum nitride composite. Composites Part A Applied Science and Manufacturing 2002;33:289-92.

[235] Gu J, Zhang Q, Dang J, Zhang J, Yang Z. Thermal conductivity and mechanical properties of aluminum nitride filled linear low density polyethylene composites. Polymer Engineering \& Science 2009:49:1030-4.

[236] Agari Y, Ueda A, Nagai S. Thermal conductivities of composites in several types of dispersion systems. Journal of Applied Polymer Science 1991; $42: 1665-9$.

[237] Kochetov R, Korobko AV, Andritsch T, Morshuis PHF, Picken SJ, Smit JJ. Modelling of the thermal conductivity in polymernanocom posites and the impact of the interface between filler and matrix. Journal of Physics D 2011;44:395401/1-395401.

[238] Naffakh M, Diez-Pascual AM, Marco C, Ellis G. Morphology and thermal properties of novel poly(phenylene sulfide) hybrid nanocomposites based on single-walled carbon nanotubes and inorganic fullerene-like $\mathrm{WS}_{2}$ nanoparticles. Journal of Materials Chemistry 2012:22:1418-25.

[239] Díez-Pascual AM, Naffakh M, Gómez-Fatou MA. Characterization and modelling of the properties of poly(ether ether ketone)/carbon nanotube/inorganic fullerene-like WS2 hybrid nanocomposites. Materials Chemistry and Physics 2011;130:126-33.
[240] Nejah SJ. A review on modelling on the thermal conductivity of polymeric nanocomposites. E-Polymers 2012;25:1-38.

[241] Diez-Pascual AM, Guang J, Simard B, Gómez-Fatou MA Poly(phenylene sulphide) and poly(ether ether ketone) composites reinforced with single-walled carbon nanotube buckypaper. II-Mechanical properties, electrical and thermal conductivity. Composites Part A Applied Science and Manufacturing 2012:43:1007-15.

[242] Díez-Pascual AM, Naffakh M, Gómez MA, Marco C, Ellis G, Martínez MT, Anson A, Gonzalez-Dominguez JM, Martinez-Rubi Y, Simard B. Development and characterization of PEEK/carbon nanotube composites. Carbon 2009;47:3079-90.

[243] Maxwell JC. A treatise on electricy and magnetism. 3rd ed. New York: Dover: 1954, 1094 pp.

[244] Naffakh M, Marco C, Ellis G. Novel polypropylene/inorganic fullerene-like $\mathrm{WS}_{2}$ nanocomposites containing a $\beta$-nucleating agent: dynamic crystallization and melting behaviour. Journal of Physical Chemistry B 2011;115:10836-43.

[245] Zhao S, Xu N, Xin Z, Jiang C. A novel highly efficient $\beta$-nucleating agent for isotactic polypropylene. Journal of Applied Polymer Science 2012;123:108-17.

[246] Tordjeman Ph, Robert C, Marin G, Gerard P. The effect of alpha, beta crystalline structure on the mechanical properties of polypropylene. European Physical Journal E 2001:4:459-65.

[247] Mirjalili F, Chuah L, Dayang AB, Hasmaliza M, Aghababazadeh R, Ahmadum F. Mechanical properties of $\alpha-\mathrm{Al}_{2} \mathrm{O}_{3} / \mathrm{PP}$ nanocomposite. Journal of Applied Science 2009;9:3199-201.

[248] Youxinga $C$, Zhangpingb W, Yujie J. Modified polypropylene filled with nano- $\mathrm{Al}_{2} \mathrm{O}_{3}$. Advanced Materials Research 2011:174:494-7.

[249] Li L, Dou Q. Effect of malonic acid treatment on crystal struc ture, melting behavior, morphology, and mechanical properties of isotactic polypropylene/nano- $\mathrm{CaCO} 3$ composites. Journal of Macromolecular Science R Part B Physics 2011;50:831-45.

[250] Altan M, Yildirim H, Uysal A. Tensile properties of polypro pylene/metal oxide nanocomposites. Journal of Science and Technology $2001 ; 1: 25-30$.

[251] Eiras D, Pessan LA. Mechanical properties of polypropylene/calcium carbonate nanocomposites. Materials Research 2009;12:517-22.

[252] Friedrich K. Crazes and shear bands in semi-crystalline thermoplastics. Advanced Polymer Science 1983;52-53:225-73.

[253] Garcia M, Van-Vliet G, Jain S, Schrauwen BAG, Sarkissov A, VanZyl WE, Boukamp B. Polypropylene/SiO, nanocomposites with improved mechanical properties. Reviews on Advanced Materials Science 2004;6:169-75.

[254] Rong MZ, Zhang MQ, Zheng YX, Zeng HM, Walter R, Friedrich K. Structure-property relationships of irradiation grafted nanoinorganic particle filled polypropylene composites. Polymer 2001:42:167-83.

[255] Cioni B, Lazzeri A. The role of interfacial interactions in the toughening of precipitated calcium carbonate-polypropylene nanocomposites. Composite Interfaces 2010;17:543-9.

[256] Zhou R], Burkhart T. Polypropylene $/ S_{i} \mathrm{O}_{2}$ nanocomposites filled with different nanosilicas: thermal and mechanical properties, morphology and interphase characterization. Journal of Materials Science 2011;46:1228-38.

[257] Boyd RH. Relaxation processes in crystalline polymers: experimental behaviour-a review. Polymer 1985;26:323-47.

[258] Ito M, Mizuochi K, Kanamoto T. Effects of crystalline forms on the deformation behaviour of nylon-6. Polymer 1998;39:4593-8.

[259] Ning $X$, Ishida $H$. Dynamic mechanical analysis of RIM nylon6. Journal of Polymer Science Part B Polymer Physics 1991;29: 1479-92.

[260] Le-Huy HM, Rault J. Remarks on the $\alpha$ and $\beta$ transitions in swollen polyamides. Polymer 1994;35:136-9.

[261] Cayer-Barrioz J, Ferry L, Frihi D, Cavalier K, Séguéla R, Vigier G. Microstructure and mechanical behavior of polyamide 66 precipitated calcium carbonate composites: influence of the particle surface treatment. Journal of Applied Polymer Science 2006;100:989-99.

[262] Li Y, Yu J, Guo ZX. The influence of silane treatment on nylon$6 /$ nano-SiO $_{2}$ in situ polymerization. Journal of Applied Polymer Science 2002:84:827-34.

[263] Zheng LY, Lau KT, Zhao LX, Zhang YQ, Hui D. Mechanical and thermal properties of nanoAl $\mathrm{O}_{3}$ /nylon 6 composites. Chemical Engineering Communications 2010;197:343-51.

[264] Garcia M, Van-Vliet G, Ten-Cate MG], Chavez F, Norder B, Kooi $B$, Van-Zyl WE, Verweij $H$, Blank DHA. Large scale extrusion processing and characterization of hybrid nylon $6 / \mathrm{SiO}_{2}$ nanocomposites. Polymers for Advanced Technologies 2004;15:164-72. 
[265] Zuev VV, Ivanova YG. Mechanical and electrical properties of polyamide-6-based nanocomposites reinforced by fulleroid fillers. Polymer Engineering \& Science 2012;52:1206-11.

[266] Díez-Pascual AM, Naffakh M, Marco C, Ellis G. Mechanical and electrical properties of carbon nanotube/poly(phenylene sulphide) composites incorporating polyetherimide and inorganic fullerene-like nanoparticles. Composites Part A Applied Science and Manufacturing 2012;43:603-12.

[267] Lu SX, Cebe P. Effects of annealing on relaxation behavior and charge trapping in film processed poly(phenylene sulfide). Journal of Applied Polymer Science 1996;61:473-83.

[268] Wang X, Tong W, Li W, Huang H, Yang J, Li G. Preparation and properties of nanocomposite of poly(phenylene sulfide)/calcium carbonate. Polymer Bulletin 2006:57:953-62.

[269] Christian J, Schwartz A, Bahadur S. Studies on the tribolog ical behavior and transfer film-counterface bond strength for polyphenylene sulfide filled with nanoscale alumina particles. Wear 2000:237:261-73.

[270] Bahadur S, Sunkara C. Effect of transfer film structure, composi tion and bonding on the tribological behavior of polyphenylene sulfide filled with nanoparticles of $\mathrm{TiO}_{2}, \mathrm{ZnO}, \mathrm{CuO}$ and $\mathrm{SiC}$. Wear 2005:258:1411-21.

[271] Joseph S, Bambole VA, Mahanwar PA. Mechanical properties of poly(ether ether ketone) composites reinforced by carbon nanoplatelets and nanoalumina. Journal of Thermoplastic Composite Materials 2011:24:755-65.

[272] Knör N, Gebhard A, Haupert F, Schlarb AK. Polyetheretherketone (PEEK) nanocomposites for extreme mechanical and tribological loads. Mechanics of Composite Materials 2009:45:199-206.

[273] Knör N, Walter R, Haupert F. Mechanical and thermal properties of nano-titanium dioxide reinforced polyetheretherketone produced by optimized twin screw extrusion. Journal of Thermoplastic Composite Materials 2011:24:185-205.

[274] Zhou B, Ji X, Sheng Y, Wang L, Jiang Z. Mechanical and thermal properties of poly-ether ether ketone reinforced with $\mathrm{CaCO}_{3}$. European Polymer Journal 2004;40:2357-63.

[275] Guoliang P, Qiang G, Aiguo T, Zhiqiang H. Mechanical behaviors of $\mathrm{Al}_{2} \mathrm{O}_{3}$ nanoparticles reinforced polyetheretherketone. Materials Science and Engineering A 2008;492:383-91.

[276] Goyal RK, Tiwari AN, Negi YS. Role of interface on dynamic modulus of high performance poly(ether ether ketone)/ceramic composites. Journal of Applied Polymer Science 2011;121:436-44.

[277] Goyal RK, Tiwari AN, Negi YS. High performance nanocomposites for tribological applications: preparation and characterization. Materials Science and Engineering A 2008:486:602-10.

[278] Zhang Z, Friedrich K. Tribological characteristics of micro- and nanoparticle filled polymer composites. In: Friedric K, Fakirov S, Zhang Z, editors. Polymer composite-from nano- to macro-scale. Berlin: Springer; 2005. p. 269-90.

[279] Rapoport L, Nepomnyashchy O, Lapsker I, Verdyan A, Moshkovich A, Feldman $Y$, Tenne R. Behavior of fullerene-like $\mathrm{WS}_{2}$ nanoparticles under severe contact conditions. Wear 2005;259:703-7.

[280] Huang CK, Li XY. Study on kneading and molding of $\mathrm{PP} / \mathrm{TiO}_{2}$ nanocomposite. International Polymer Processing 2008;24: 7-9.

[281] Nien MH, Lin JC. Investigation of friction and wear behaviors for a silica/polypropylene nanocomposites. 17th International Conference on Composites or Nano Engineering, ICCE-17 Honolulu, Hawaii (USA), http://wjoe.hebeu.edu.cn/ICCE-17\%20proceedings \%20Hawaii\%20USA/Nien,\%20M.H.\%20(Tatung\%20U.\%20Taipei) $\% 20 \% 20749$.pdf [accessed March 2013]; 2009. 2 pp.

[282] Sreekala MS, Lehman B, Friedrich K, Rong MZ. Nanosilica reinforced polypropylene nanocomposites: microstructural analysis and crystallization behaviour. International Journal of Polymer Materials 2006:55:577-94.

[283] Dasari A, Yu ZZ, Mai YW. Effect of surfactant on wear of polymer nanocomposites. 16th International Conference on Composite Materials, Kyoto, Japan, http://www.iccm-central.org/ Proceedings/ICCM16proceedings/contents/pdf/WedA/WeAA102ge_yuz225927.pdf [accessed March 2013]; 2007. 1 pp.

[284] Garcia M, De-Rooij M, Winnubst L, Van-Zyl WE, Verweij HJ. Friction and wear studies on Nylon $6 / \mathrm{SiO}_{2}$ nanocomposites. Journal of Applied Polymer Science 2004;92:1855-62.

[285] Zhao LX, Zheng LY, Zha SG. Tribological performance of nano$\mathrm{Al}_{2} \mathrm{O}_{3}$ reinforced polyamide 6 composites. Materials Letters 2006:60:2590-3.

[286] Pesetskii S, Bogdanovich SP, Myshkin NK. Tribological behavior of nanocomposites produced by the dispersion of nanofillers in poly mer melts. Journal of Friction and Wear 2007;28:457-75.
[287] Díez-Pascual AM, Naffakh M, Marco C, Ellis G. Rheological and tribological properties of carbon nanotube/thermoplastic nanocomposites incorporating inorganic fullerene-like WS2 nanoparticles. Journal of Physical Chemistry B 2012;116:7959-69.

[288] Friedrich K, Zhang Z Schlarb AK. Effects of various fillers on the sliding wear of polymer composites. Composites Science and Technology 2005;65:2329-43.

[289] Mccook NL, Hamilton MA, Burris DL, Sawyer WG. Tribological results of PEEK nanocomposites in dry sliding against $440 \mathrm{C}$ in various gas environments. Wear 2007:262:1511-5.

[290] Wang Q Xu J, Shen W, Liu W. An investigation of the fric tion and wear properties of nanometer $\mathrm{Si}_{3} \mathrm{~N}_{4}$ filled PEEK. Wear 1996;196:82-6.

[291] Wang Q Xue Q Liu H, Shen W, Xu J. The effect of particle size of nanometer $\mathrm{ZrO}_{2}$ on the tribological behaviour of PEEK. Wear 1996:198:216-9.

[292] Wang Q, Xue Q, Shen W, Wang Q, Xue Q, Shen W. The friction and wear properties of nanometre $\mathrm{SiO}_{2}$ filled polyetheretherketone. Tribology International 1997;30:193-7.

[293] Wang Q Xu J, Shen W, Xue Q. The effect of nanometer SiC filler on the tribological behavior of PEEK. Wear 1997;209:316-21.

[294] Xu J, Wang Q. Wear mechanisms of polyetheretherketone composites filled with various kinds of SiC. Wear 1997;213:54-8.

[295] Grein C. Toughness of neat, rubber modified and filled $\beta$-nucleated polypropylene: from fundamentals to applications. Advances in Polymer Science 2005;188:43-104.

[296] Thierry A, Straupé C, Wittmann JC, Lotz B. Organogelators and polymer crystallization. Macromolecular Symposia 2006;241:103-10.

[297] Libster D, Aserin A, Garti N. Advanced nucleating agents for polypropylene. Polymer for Advanced Technologies 2007;18: 685-95.

[298] Varga J. $\beta$-modification of isotactic polypropylene: preparation, structure, processing, properties, and application. Journal of Macromolecular Science Part B Physics 2002:41:1121-71.

[299] Gahleitner M, Grein C, Kheirandish S, Wolfschwenger ]. Nucleation of polypropylene homo- and copolymers. International Polymer Processing 2011:16:2-20.

[300] Thierry A, Straupé C, Lotz B, Wittmann JC. Physical gelation. A path towards ideal dispersion of additives in polymers. Polymer Communications 1990;31:299-301.

[301] Shephard TA, Delsorbo CR, Louth RM, Walborn JL, Norman DA, Harvey NG, Spontak RJ. Self-organization and polyolefin nucleation efficacy of 1,3:2,4-di-p-methylbenzylidene sorbitol. Journal of Polymer Science Part B Polymer Physics 1997;35:2617-28.

[302] Bauer T, Thomann R, Mülhaupt R. Two-component gelators and nucleating agents for polypropylene based upon supramolecular assembly. Macromolecules 1998;31:7651-8.

[303] Vidotto G, Levy D, Kovacs AJ. Cristallisation et fusion des polymères autoensemencés. I Polybutène-1, polyéthylene et polyoxyéthylène de haute masse moléculaire. Kolloid-Zeitschrift und Zeitschrift für Polymere 1969;230:289-305.

[304] Legras R, Mercier JP, Nield E. Polymer crystallization by chemical nucleation. Nature 1983;304:432-4.

[305] Manias E, Touny A, Wu L, Strawhecker K, Lu B, Chung TC. Polypropylene/montmorrillonite nanocomposites. Review of the synthetic routes and material properties. Chemistry of Materials 2001;13:3516-23.

[306] Manchado MAL, Valentini L, Biagiotti J, Kenny JM. Thermal and mechanical properties of single-walled carbon nanotubespolypropylene composites prepared by melt processing. Carbon 2005:43:1499-505.

[307] Marco C, Naffakh M, Gómez MA, Santoro G, Ellis G. The crystallization of polypropylene in multiwall carbon nanotube-based composites. Polymer Composites 2011:32:324-33.

[308] Naffakh M, Remškar M, Marco C, Gómez-Fatou MA, Jiménez I. Towards a new generation of polymer nanocomposites based on inorganic nanotubes. Journal of Materials Chemistry 2011;21:3574-8.

[309] Chen B, Evans JRG. Impact strength of polymer-clay nanocomposites. Soft Matter 2009:5:3572-84.

[310] Ghugare SV, Govindaiah P, Avadhani CV. Polypropylene-organoclay nanocomposites containing nucleating agents. Polymer Bulletin 2009:63:897-909.

[311] Naffakh M, Díez-Pascual AM, Gómez-Fatou MA. New hybrid nanocomposites containing carbon nanotubes, inorganic fullerenelike $W_{2}$ nanoparticles and poly(ether ether ketone)(PEEK). Journal of Materials Chemistry 2011:21:7425-33.

[312] Padden FJ, Keith HD. Spherulitic crystallization in polypropylene. Journal of Applied Physics 1959:30:1479-84. 
[313] Keith HD, Padden FJ, Walter NM, Wyckoff HW. Evidence of a second crystal form of polypropylene. Journal of Applied Physics 1959;30:1485-8.

[314] Brückner S, Meille SV, Petraccone V, Pirozzi B. Polymorphism in isotactic polypropylene. Progress in Polymer Science 1991; 16:361-404

[315] Lotz B, Wittmann JC, Lovinger AJ. Structure and morphology of poly(propylenes): a molecular analysis. Polymer $1996: 37: 4979-92$

[316] Auriemma F, De-Rosa C, Corradini P. Solid mesophases in semicrystalline polymers: structural analysis by diffraction techniques. Advances in Polymer Science 2005;181:1-74.

[317] Androsch R, Di-Lorenzo ML, Schick C, Wunderlich B. Mesophases in polyethylene, polypropylene, and poly(1-butene). Polymer 2010;51:4639-62.

[318] Jacoby P, Bersted BH, Kissel W], Smith CE. Studies on the $\beta$ crystalline form of isotactic polypropylene. Journal of Polyme Science Part B Polymer Physics 1986;24:461-91.

[319] Tjong SC, Shen JS, Li RKY. Mechanical behavior of injection molded $\beta$-crystalline phase polyp ropylene. Polymer Engineering \& Science $1996: 36: 100-5$

[320] Karger-Kocsis ], Varga ]. Effects of $\beta$ - $\alpha$ transformation on the static and dynamic tensile behavior of isotactic polypropylene. Journal of Applied Polymer Science 1996;62:291-300.

[321] Sterzynski T, Oysaed H. Structure modification of isotactic polypropylene by bi-component nucleating system. Polymer Engineering \& Science 2004;44:352-61.

[322] Zhang YF, Xin Z. Isothermal crystallization behaviour of isotactic polypropylene nucleated with $\alpha / \beta$ compounding nucleating agents. Journal of Polymer Science Part B Polymer Physics 2007:45:590-6.

[323] Zhang YF. Crystallization and melting behaviors of isotactic polypropylene nucleated with compound nucleating agents. Journal of Polymer Science Part B Polymer Physics 2008:46:911-6.

[324] Bai H, Wang Y, Liu L, Zhang J, Han L. Non isothermal crystallization behaviors of polypropylene with $\alpha / \beta$ nucleating agents. Journal of Polymer Science Part B Polymer Physics 2008:46:1853-67.

[325] Zhao S, Xin Z. Nucleation characteristics of the $\alpha \beta$ compounded nucleating agents and their influences on the crystallization behavior and mechanical properties of isotactic polypropylene. Journal of Polymer Science Part B Polymer Physics 2010;48: $653-5$.

[326] Naffakh M, Marco C, Ellis G. Novel polypropylene/inorganic fullerene-like $\mathrm{WS}_{2}$ nanocomposites containing a $\boldsymbol{\beta}$-nucleating agent: isothermal crystallization and melting behavior. Journal of Physical Chemistry B 2011;116:1788-95

[327] Prachum Y, Strauss RHA, Kiatkamjornwong S. The physi cal and mechanical properties of beta-nucleated polypropylene/montmorillonite nanocomposites. Journal of Applied Polymer Science 2011;122:1066-76

[328] Zhang N, Zhang Q, Wang K, Deng H, Fu Q. Combined effect of $\beta$-nucleating agent and multi-walled carbon nanotubes on polymorphic composition and morphology of isotactic polypropylene. Journal of Thermal Analysis and Calorimetry 2012;107:733-43.

[329] Wang S, Yang W, Bao R, Wang B, Xie B, Yang M. The enhanced nucleating ability of carbon nanotube-supported $\beta$-nucleating agent in isotactic polypropylene. Colloid \& Polymer Science 2010;288:61-88.

[330] Zhang Z, Chen C, Wang C, Junping Z, Mai K. A novel highly efficient $\beta$-nucleating agent for polypropylene using nano- $\mathrm{CaCO}_{3}$ as a support. Polymer International 2010;59:1199-204.

[331] Ijima S. Helical microtubules of graphitic carbon. Nature 1991:354:56-8.

[332] Dresselhaus MS, Dresselhaus G, Avourios P. Carbon nanotubes. Topics in Applied Physics 2001;80:1-9.

[333] Avouris P. Molecular electronics with carbon nanotubes. Accounts of Chemical Research 2002:35:1026-34.

[334] Arico AS, Bruce P, Scrosati B, Tarascon JM, Van-Schalkwijk W. Nanostructured materials for advanced energy conversion and storage devices. Nature Materials 2005;4:366-77.

[335] Pandey P, Datta M, Malhotra BD. Prospects of nanomaterials in biosensors. Analytical Letters 2008;41:159-209.

[336] Martin CR, Kohli P. The emerging field of nanotube biotechnology. Nature Review Drug Discovery 2003;2:29-37.

[337] Coleman JN, Khan U, Gunko YK. Mechanical reinforcement of polymers using carbon nanotubes. Advanced Materials 2006:18:689-706

[338] Ajayan PM, Schadler LS, Braun PV. Nanocomposite science and technology. Weinheim: Wiley-VCH; 2003. p. 77-80.
[339] Sahoo NG, Rana S, Cho JW, Li L, Chan SH. Polymer nanocomposites based on functionalized carbon nanotubes. Progress in Polymer Science 2010;35:837-67.

[340] Ma P, Siddiqui NA, Marom G, Kim J. Dispersion and functionalization of carbon nanotubes for polymer-based nanocomposites: a review. Composites Part A Applied Science and Manufacturing 2010;41:1345-67.

[341] Bose S, Khare RA, Moldenaers P. Assesing the stregths and weakness of various types of pre-treatments of carbon nanotubes on the properties of polymer/carbon nanotubes composites: a critical review. Polymer 2010;51:975-93.

[342] Isayev AI, Kumar R, Lewis T. Ultrasound assited twin screw extrusion of polymer-nanocomposites containing carbon nanotubes. Polymer 2009:50:250-60.

[343] Yu C, Wu D, Liu Y, Qiao H, Yu Z, Dasari A, Du X, Mai Y. Electrical and dielectric properties of polypropylene nanocomposites based on carbon nanotubes and barium titanate nanoparticles. Composites Science and Technology 2011:71:1706-12

[344] Zhang Y, Lin Y, Chang C, Wu T. Conducting and magnetic behaviors of polyaniline coated multi-walled carbon nanotube composites conataining monodispersed magnetite nanoparticles. Synthetic Metals 2011;161:937-42.

[345] Kedem S, Schmidt J, Paz Y, Cohen Y. Composite polymer nanofibers with carbon nanotubes and titanium dioxide particles. Langmuir $2005 ; 21: 5600-4$.

[346] Reddy KR, Sin BC, Ryu KS, Kim J, Chung H, Lee Y. Conducting polymer functionalized multi-walled carbon nanotubes with noble meta nanoparticles: synthesis, morphological characteristics and elec trical properties. Synthetic Metals 2009:159:595-603.

[347] Sepahvand R, Adeli M, Astinchap B, Kabiri R. New nanocompos ites containing metal nanoparticles, carbon nanotube and polymer. Journal of Nanoparticle Research 2008;10:1309-18.

[348] Fortunati E, Dángelo F, Martino S, Orlacchio A, Kenny JM, Armentano I. Carbon nanotubes and silver nanoparticles for multifunctional conductive biopolymer composites. Carbon 2011;49:2370-9.

[349] Rangari VK, Mohammad GM, Jeelani S, Hundley A, Vig K, Singh SR, Pillai S. Synthesis of Ag/CNT hybrid nanoparticles and fabrication of their nylon-6 polymer nanocomposite fibers for antimicrobial applications. Nanotechnology 2010;21:095102/1-95102.

[350] Hsieh TH, Kinloch AJ, Taylor AC, Sprenger S. The effect of silica nanoparticles and carbon nanotubes on the toughness of a thermosetting epoxy polymer. Journal of Applied Polymer Science 2011;119:2135-42

[351] Sun D, Chu C, Sue H. Simple approach for preparation of epoxy hybrid nanocomposites based on carbon nanotubes and modelclay. Chemistry of Materials 2010;22:3773-8

[352] Diez-Pascual AM, Naffakh M, Gonzalez-Dominguez JM, Ansón A, Martínez-Rubí Y, Martinez MT, Simard B, Gómez MA. High performance PEEK/carbon nanotube composites compatibilized with polysulfones-I: structure and thermal properties. Carbon 2010:48:3485-99.

[353] Naffakh M, Marco C, Gomez MA, Ellis G, Maser WK, Benito A Martinez MT. Crystalline transformations in nylon-6/single-walled carbon nanotube nanocomposites. Journal of Nanoscience and Nanotechnology 2009:9:1-7.

[354] Offringa AR. Thermoplastic composites in aerospace-proven through cost effective processing. In: 41 th International conference on automated composites (ICAC). 1995, p. 161-9.

[355] Marsh G. Next step for automotive materials. Materials Today $2003: 6: 36-43$.

[356] Tong L, Mouritz AP, Bannister M. 3D fibre reinforced polymer composites. Oxford: Elsevier Science; 2002. p. 1-12.

[357] Steeves CA, Fleck NA. In-plane properties of composite laminates with through-thickness pin reinforcement. International Journal of Solids and Structures 2006:43:3197-212

[358] Chang P, Mouritz AP, Cox BN. Flexural properties of z-pinned laminates. Composites Part A Applied Science and Manufacturing 2007:38:244-51.

[359] Khan SU, Kim JK. Impact and delamination failure of multi scale carbon nanotube-fiber reinforced polymer composites: a review. International Journal of Aeronautical and Space Sciences $2011 ; 12: 115-33$

[360] Qian H, Greenhalgh ES, Shaffer MSP, Bismarck A. Carbon nanotube based hierarchical composites: a review. Journal of Materials Chemistry 2010;20:4751-62.

[361] Inam F, Wong DWY, Kuwata M, Peijs T. Multiscale hybrid micronanocomposites based on carbon nanotubes and carbon fibers. Journal of Nanomaterials 2010;2010:453420/1-453420. 
[362] Lin G, Xie G, Sui G, Yang R. Hybrid effect of nanoparticles with carbon fibers on the mechanical and wear properties of polymer composites. Composites Part B Engineering 2012;43:44-9.

[363] Diez-Pascual AM, Naffakh M. Tuning the properties of carbon fiberreinforced poly(phenylene sulphide) laminates via incorporation of inorganic nanoparticles. Polymer 2012;53:2369-78.

[364] Mahfuz H, Powell F, Granata R, Hosur M, Khan M. Coating of carbon fiber with polyhedral oligomeric silsesquioxane (POSS) to enhance mechanical properties and durability of carbon/vinyl ester composites. Materials 2011:4:1619-31.

[365] Iqbal K, Khan SU, Munir A, Kim JK. Impact damage resistance of CFRP with nanoclay-filled epoxy matrix. Composites Science and Technology 2009;69:1949-57.

[366] Rapoport L, Fleischer N, Tenne R. Fullerene-like $\mathrm{WS}_{2}$ nanoparticles: superior lubricants for harsh conditions. Advanced Materials 2003:15:651-5.

[367] Xian G, Zhang Z, Friedrich K. Tribological properties of micro and nanoparticles-filled poly(etherimide) composites. Journal of Applied Polymer Science 2006;101:1678-86.

[368] Hussain M, Nakahira A, Nishijima S, Niihara K. Evaluation of machanical behaviour of CFRC transverse to the fiber direction at room and cryogenic temperature. Composites Part A Applied Science and Manufacturing 2000;31:173-9.

[369] TimmermanJF, Hayes BS, Seferis JC. Nanoclay reinforcement effects on the cryogenic microcraking of carbon fiber/epoxy composites. Composites Science and Technology 2002;62:1249-58.
[370] Genuth I, Yaffe T. Protecting the soldiers of tomorrow. IsraCast-Israeli Technology News. http://www.isracast.com/ results.aspx?text=nano-armor [accessed March 2013]; 2006 (February).

[371] Adini AR, Redlich M, Tenne R. Medical applications of inorganic fullerene-like nanoparticles. Journal of Materials Chemistry 2011;21:15121-31.

[372] Katz A, Redlich M, Rapoport L, Wagner HD, Tenne R. Self-lubricating coatings containing fullerene-like WS $\mathrm{W}_{2}$ nanoparticles for orthodontic wires and other possible medical applications. Tribology Letters 2006;21:135-9.

[373] Adini AR, Feldman Y, Cohen SR, Rapoport L, Moshkovith A, Redlich $M$, Moshonov Y, Shay B, Tenne R. Alleviating incidental and fatiguerelated failure of NiTi root canal files by self-lubricating coating. Journal of Materials Research 2011;26:1234-42.

[374] Tahir MN, Yella A, Sahoo JK, Natalio F, Kolb U, Jochum F, Theato P, Tremel W. IF-ReS 2 with covalently linked porphyrin antennae. Israel Journal of Chemistry 2010;50:500-5.

[375] Sahoo JK, Tahir MN, Yella A, Branscheid R, Kolb U, Tremel W. Soluble IF-ReS 2 nanoparticles by surface functionalization with terpyridine ligands. Langmuir 2011;27:385-91.

[376] Yadgarov L, Rosentsveig R, Leitus G, Albu-Yaron A, Moshkovith A, Perfilyev V, Vasic R, Frenkel AI, Enyashin AN, Seifert G, Rapoport $\mathrm{L}$, Tenne R. Controlled doping of $\mathrm{MS}_{2}(\mathrm{M}=\mathrm{W}$, Mo) nanotubes and fullerene-Like nanoparticles. Angewandte Chemie International Edition 2012:51:1148-51. 